\title{
WATER-RESOURCES APPRAISAL OF THE LOWER BRULE INDIAN RESERVATION IN CENTRAL SOUTH DAKOTA
}

U.S. GEOLOGICAL SURVEY

Water-Resources Investigations Report 95-4116

Prepared in cooperation with the LOWER BRULE SIOUX TRIBE

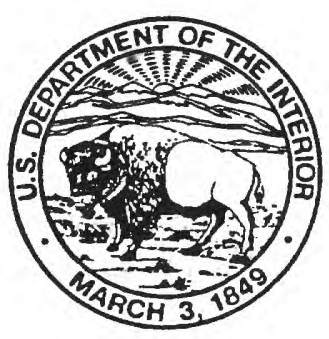




\section{WATER-RESOURCES APPRAISAL OF THE \\ LOWER BRULE INDIAN RESERVATION \\ IN CENTRAL SOUTH DAKOTA}

By Kimberly A. Ogle

U.S. GEOLOGICAL SURVEY

Water-Resources Investigations Report 95-4116

Prepared in cooperation with the

LOWER BRULE SIOUX TRIBE

Rapid City, South Dakota

1995

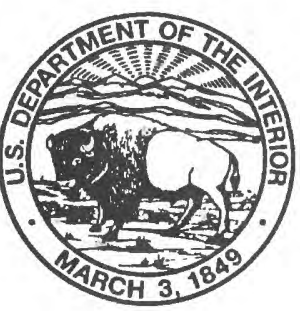




\section{U.S. DEPARTMENT OF THE INTERIOR \\ BRUCE BABBITT, Secretary \\ U.S. GEOLOGICAL SURVEY \\ Gordon P. Eaton, Director}

For additional information write to:

District Chief

U.S. Geological Survey

1608 Mt. View Road

Rapid City, SD 57702
Copies of this report can be purchased from:

U.S. Geological Survey Earth Science Information Center Open-File Reports Section Box 25286, MS 517 Denver Federal Center Denver, CO 80225 


\section{CONTENTS}

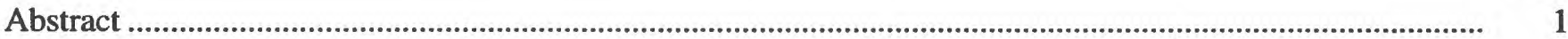

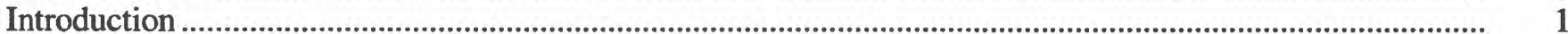

Purpose and scope

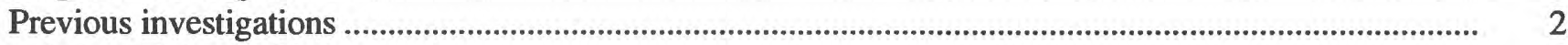

Description of the study area.......................................................................................................................

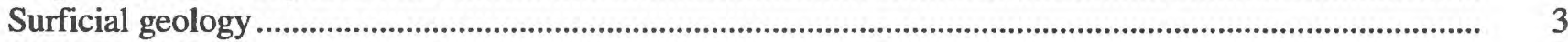

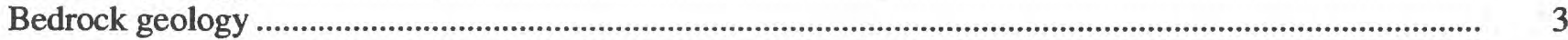

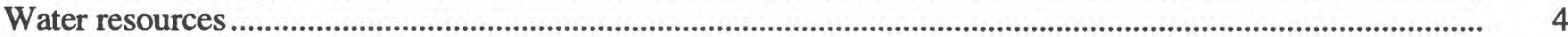

Surface-water availability and quality ........................................................................................................ 4

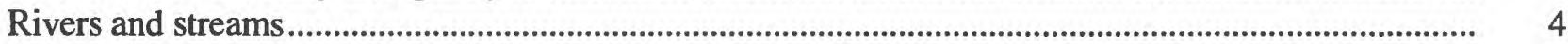

Stock ponds and dugouts ........................................................................................................... 11

Ground-water availability and water quality ............................................................................................ 14

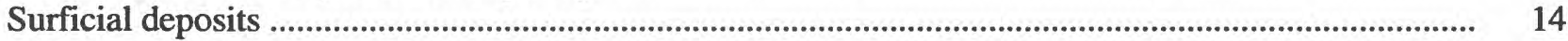

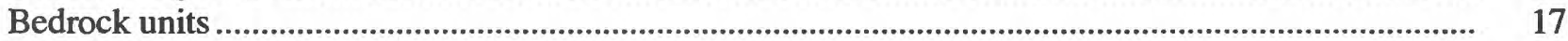

Well inventory ......................................................................................................................................... 23

Indian water rights........................................................................................................................................ 23

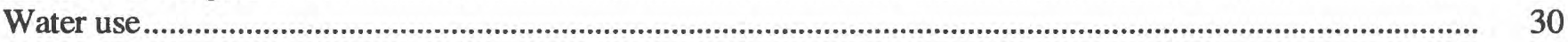

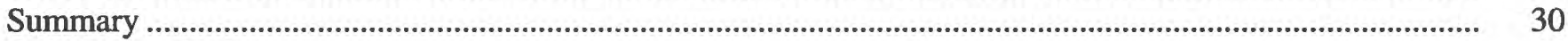

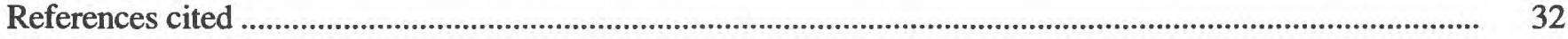

Supplemental information ..................................................................................................................... 33

\section{ILLUSTRATIONS}

Figure 1. Map showing location of study area ............................................................................................. 2

2. Map showing generalized surficial geology on the Lower Brule Indian Reservation................... 4

3. Diagram showing generalized average annual hydrologic budget for the Lower Brule Indian Reservation

4-11. Maps showing:

4. Locations of stock ponds and dugouts on the Lower Brule Indian Reservation visited in February 1993

5. Locations of additional stock ponds and dugouts on the Lower Brule Indian Reservation from aerial photographs taken in August and September 1991

6. Locations of stock dams that were breached or possibly breached on the Lower Brule Indian Reservation as of 1993.

7. Ground-water availability from surficial deposits on the Lower Brule Indian Reservation.

8. Hydrogeology of the Dakota Sandstone on the Lower Brule Indian Reservation................. 19

9. Hydrogeology of the Inyan Kara Group on the Lower Brule Indian Reservation.................. 20

10. Hydrogeology of the Minnelusa Formation on the Lower Brule Indian Reservation ........... 21

11. Hydrogeology of the Madison Group on the Lower Brule Indian Reservation...................... 22

12. Hydrographs of water levels in wells completed in the Dakota Sandstone .................................... 22

13. Map showing water types in the Dakota Sandstone on the Lower Brule Indian Reservation as of 1972

14. Map showing locations of usable wells on the Lower Brule Indian Reservation as of 1992 


\section{TABLES}

Table 1. Generalized stratigraphic chart of geologic units present in the Lower Brule

Indian Reservation

2. Storage capacities of Missouri River reservoirs adjacent to or upstream from the Lower Brule Indian Reservation.

3. Annual Missouri River discharge at Oahe and Big Bend Dams, 1968-92.

4. Estimated average annual streamflow and peak flows for given recurrence intervals for selected streams on the Lower Brule Indian Reservation

5. Summary of chemical analyses of water from the Missouri River at Pierre (station 06440000) before filling of Lake Oahe.

6. Summary of chemical, biological, and suspended-sediment analyses of water from the Missouri River at Pierre (station 06440000) after filling of Lake Oahe

7. U.S. Environmental Protection Agency drinking-water regulations

8. Chemical analyses of water samples collected from Brule and Medicine Creeks near Lower Brule, April 1, 1971

9. Qualitative ranking of stock ponds and dugouts on the Lower Brule Indian Reservation used in the 1979 Bureau of Indian Affairs inventory.

10. Field analyses of water samples collected from stock ponds and dugouts on the Lower Brule Indian Reservation during February 1993

11. Physical and chemical data for water samples collected at seven shallow wells on the Lower Brule Indian Reservation, 1969.

12. Usable wells on the Lower Brule Indian Reservation, 1992.

13. Selected field analyses of ground-water samples collected in 1992 from wells on the Lower Brule Indian Reservation.

14. Surface-water use for irrigation on the Lower Brule Indian Reservation

15. Daily discharge for the Missouri River at Pierre (station 06440000) for water years 1934-65.

16. Daily discharge for the Missouri River at Chamberlain (station 06443000) for the periods September 1928 to September 1929 and April 1945 to September 1954

17. Ponds, stock ponds, and dugouts on the Lower Brule Indian Reservation 


\begin{tabular}{rcl}
\hline \multicolumn{1}{r}{ Multiply } & \multicolumn{1}{c}{ By } & \multicolumn{1}{c}{ To Obtain } \\
\hline acre & 0.4047 & hectare \\
acre-foot & 1,233 & cubic meter \\
acre-foot & 0.001233 & cubic hectometer \\
cubic meter per year \\
acre-foot per year & 1,233 & cubic meter per square kilo- \\
acre-foot per square mile & 476 & meter \\
& & cubic meter per second \\
cubic foot per second & 0.028317 & meter \\
foot & 0.3048 & meter per year \\
foot per year & 0.3048 & cubic meter per day \\
gallon per day & 0.003785 & liter per second \\
gallon per minute & 0.06309 & kilometer \\
mile & 1.609 & square kilometer \\
square mile & 2.590 &
\end{tabular}

Temperature can be converted to degree Fahrenheit $\left({ }^{\circ} \mathrm{F}\right)$ or degree Celsius $\left({ }^{\circ} \mathrm{C}\right)$ by the following equations:

$$
\begin{aligned}
& { }^{\circ} \mathrm{F}=9 / 5\left({ }^{\circ} \mathrm{C}\right)+32 \\
& { }^{\circ} \mathrm{C}=5 / 9\left({ }^{\circ} \mathrm{F}-32\right)
\end{aligned}
$$

Sea level: In this report, "sea level" refers to the National Geodetic Vertical Datum of 1929--a geodetic datum derived from general adjustment of the first-order level nets of the United States and Canada, formerly called Sea Level Datum of 1929.

The following terms and abbreviations also are used in this report:

$\mu \mathrm{S} / \mathrm{cm}$, microsiemens per centimeter at 25 degrees Celsius

$\mathrm{mg} / \mathrm{L}$, milligram per liter

$\mu \mathrm{g} / \mathrm{L}$, microgram per liter

col/100 mL, colonies per 100 milliliter

mm, millimeter

NTU, nephelometric turbidity units

$\mu \mathrm{m}$, micrometer 


\section{WELL-NUMBERING SYSTEM}

The well-numbering system used in this report is based on the Federal land-survey system for subdivisions of public land. Each "number" (actually number-letter designation) indicates the location of the well with respect to township (T.), range (R.), and section (sec.). Number 107N75W29CCDB indicates a well in T. 107 N., R. 75 W., sec. 29. The last four letters show the location within the section; the first letter (C) identifies the quarter section (160 acres); the second letter (C), the quarter-quarter section (40 acres); the third letter (D) the quarter-quarterquarter section (10 acres); and the fourth letter (B) the quarter-quarter-quarter-quarter section (2 1/2 acres). Well 107 N75W29CCDB is in the northwest quarter of the southeast quarter of the southwest quarter of the southwest quarter of section 29, township 107 north, range 75 west (see figure below). Where more than one well is located within a $21 / 2$-acre tract, a number is added following the letter sequence to distinguish them.

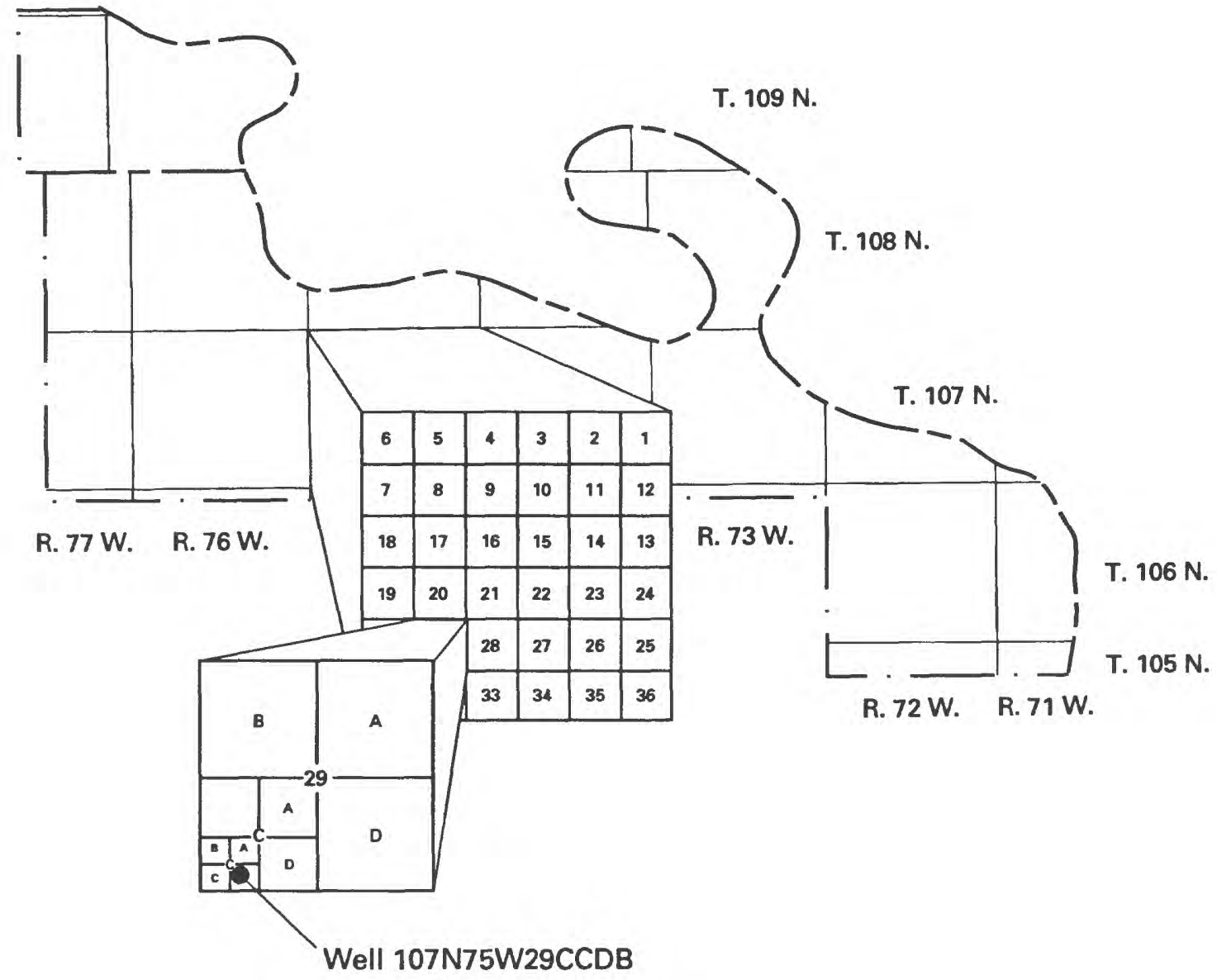




\title{
WATER-RESOURCES APPRAISAL OF THE LOWER BRULE INDIAN RESERVATION
} IN CENTRAL SOUTH DAKOTA

\author{
By Kimberly A. Ogle
}

\section{ABSTRACT}

The Lower Brule Indian Reservation covers an area of about 404 square miles. Agriculture, primarily livestock and crop production, is the major industry. In 1992, about 5,900 acres were irrigated by the Tribe and about 3,800 other acres either were being irrigated or had State permits for irrigation.

Precipitation averages about 17.4 inches per year. Diversions to the reservation from the Missouri River average about 17,000 acre-feet per year. Evapotranspiration from the land surface of the reservation averages about 17.5 inches per year.

Missouri River reservoirs adjacent to the reservation normally store about 5 million acre-feet of water. Inflow of the Missouri River to the reservation is estimated to be about 18.3 million acre-feet per year. The dissolved-solids concentration of Missouri River reservoir water adjacent to the reservation averages slightly less than $\mathbf{5 0 0}$ milligrams per liter.

All streams on the reservation other than the Missouri River are ephemeral. In 1993, 593 stock ponds and dugouts were located on ephemeral streams. Based on visits to about 10 percent of the stock ponds and dugouts, an estimated 75 percent of the ponds and dugouts were dry, overgrown with vegetation, silted in, or had breached or leaky dams.

Ground-water supplies from surficial deposits are small and are present only along major streams and at some places along the Missouri River. Water suitable for livestock and some domestic use can be obtained throughout the reservation from artesian wells that tap the Dakota Sandstone or other deeper bedrock aquifers.

The major water use on the reservation is for irrigation. In 1990 , slightly more than 17,000 acrefeet was used to irrigate land within the reservation.

\section{INTRODUCTION}

The water-resources appraisal of the Lower Brule Indian Reservation was a 3-year study, beginning in 1992, to provide a current inventory of hydrologic information to support development of a comprehensive water plan on the reservation. Specific objectives were to document and describe the existence and status of surface-water and ground-water resources, the status of water quality, and Indian water rights including historical and present water use. This information is needed by tribal leaders to develop a water plan for effective management of the reservation's water resources.

The Lower Brule Indian Reservation covers an area of about 404 square miles within Lyman and Stanley Counties in central South Dakota (fig. 1); of this, about 35 square miles are covered by major reservoirs on the Missouri River and about 201 square miles are owned in trust by the Lower Brule Sioux Tribe or by tribal members (Bureau of Indian Affairs, written commun., Mar. 23, 1994). The reservation is bounded on the north and east by Lakes Sharpe and Francis Case, which are large Missouri River impoundments formed by main-stem dams. Agriculture is the primary industry, consisting mostly of cattle ranching and crop production. In 1992, there 


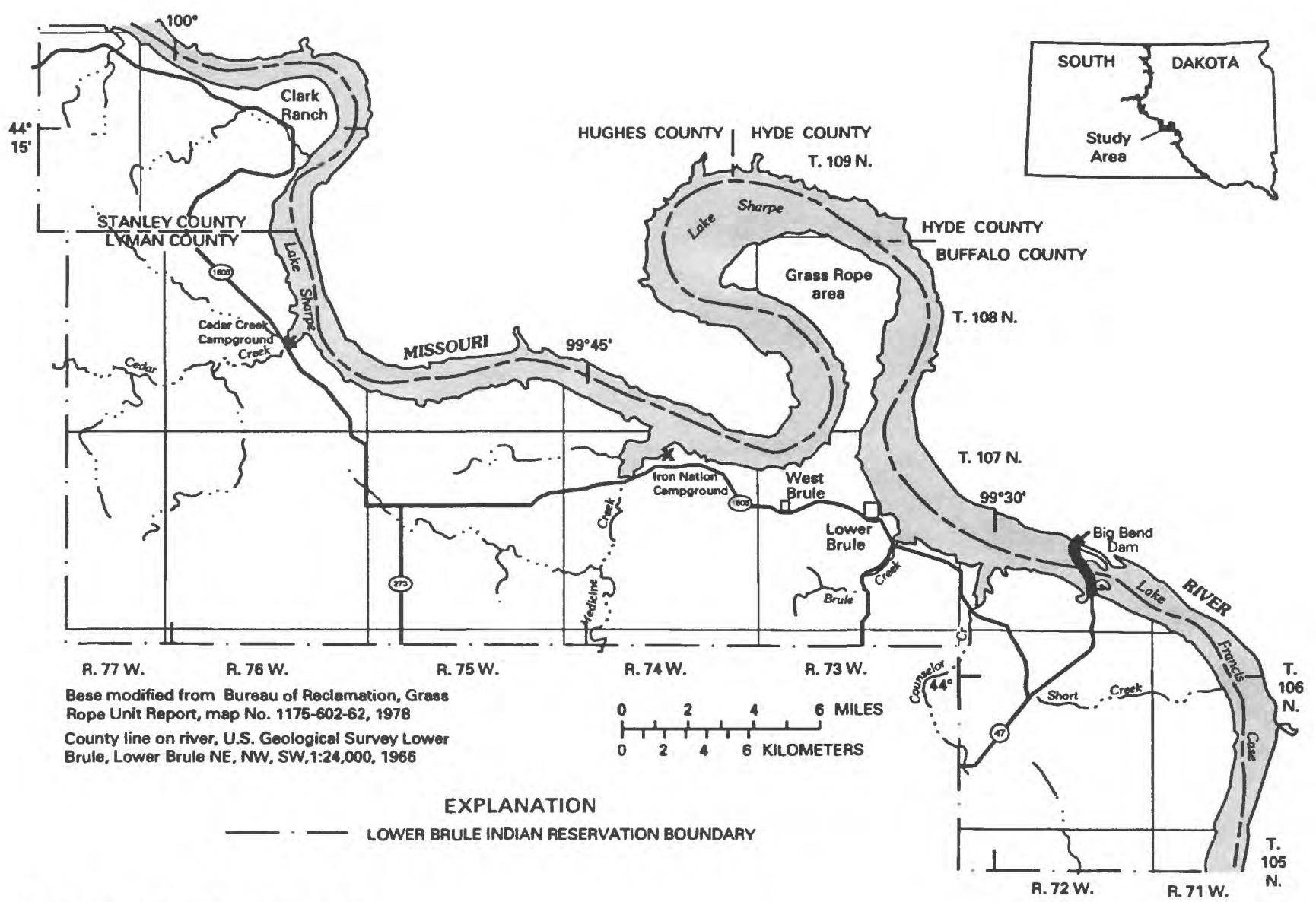

Figure 1. Location of study area.

were 5,901 acres of tribal or other Indian-owned land under irrigation (Tom Young, Bureau of Indian Affairs, oral commun., August 1993) and 3,763 acres of other land within the reservation boundary either under irrigation or for which State permits for irrigation had been issued. Other industries include hunting, fishing, a tribal propane plant, a tribal construction company, and a tribal-owned gaming casino.

\section{Purpose and Scope}

This report presents the results of: (1) compilation and analysis of existing hydrologic data; (2) an inventory of surface-water resources using 1991 aerial photographs; (3) an inventory of water wells; (4) a literature search and review, including Indian waterrights issues; and (5) compilation and analysis of water-use data for the Lower Brule Indian Reservation.

\section{Previous Investigations}

G.A. La Rocque, Jr. (1966) evaluated the general availability and depth to ground water in the Missouri River Basin. Although no site-specific data were provided, the Lower Brule Indian Reservation does lie within that study area. That report serves as a convenient guide to the approximate depth to ground water and its general availability for domestic, municipal, industrial, and irrigation use.

Howells (1974) studied the geohydrology of the Crow Creek and Lower Brule Indian Reservations of South Dakota. His investigation included the evaluation of surface-water resources (lakes, reservoirs, streams, and stock ponds); surficial geology; shallow ground-water resources; geohydrology of the bedrock aquifers; a generalized stratigraphic column describing the bedrock formations, their hydrologic characteristics, and quality of the water; and selected 
water-quality analyses for surface- and ground-water sources on the Crow Creek and Lower Brule Reservations.

Francis-Meador-Gellhaus, Inc., Consulting Engineers, of Rapid City, S. Dak., studied the quantity and quality of the water resources within the boundaries of the Lower Brule Reservation. The resulting "Phase I" report (Francis-Meador-Gellhaus, Inc., Consulting Engineers, 1978) discussed the general physiography of the reservation; water-rights issues; analyses of major drainage basins; climate; surfaceand ground-water quality; water resources; the relationship between surface water and ground water; the establishment of a hydrologic monitoring network; and future sources of water. The "Phase II" report (Francis-Meador-Gellhaus, Inc., Consulting Engineers, 1980) discussed tribal goals and objectives; economic base and projections for the reservation; land and agricultural resources; fish and wildlife resources; recreation and tourism; municipal, rural, and industrial water needs; energy aspects; watershed management; and pollution control and water quality.

\section{DESCRIPTION OF THE STUDY AREA}

The topography and surficial geology of much of the study area were shaped by the effects of continental glaciation on a land surface underlain by soft shale of marine origin. The Lower Brule Reservation is located on what was the western margin of the last continental glacial ice sheet. The ice sheet scoured the old land surface as it advanced and left a new surface as it retreated. Glaciation changed the courses of many rivers and created the Missouri River.

The formation of the Missouri River caused modification of old drainage patterns and created new ones. Huge volumes of water from melting continental ice sheets enabled the newly created Missouri River to cut its channel several hundred feet into the land surface. As the Missouri River cut its trench deeper, remnants of its valley were left behind as terraces, and tributary streams rapidly cut their channels deeper. Large volumes of shale and glacial drift were carried away by erosion, and the present "breaks" topography began to develop (Howells, 1974).

\section{Surficial Geology}

Melting glaciers left behind deposits of material, called drift, that were eroded from the surface over which the ice had moved. If the drift is an unsorted mixture of all sizes from clay to boulders, it is called till. The till on the Lower Brule Reservation generally is silty and sandy clay. Sorted drift, material that was separated by water or wind into different sizes, includes outwash, stream and lake deposits, and loess. The locations of surficial deposits within the reservation are shown in figure 2.

Because the study area is on the border of the glaciated region, much of the land surface is the Pierre Shale; only a small area is now mantled with glacial deposits. Due to the high relief and softness of the Pierre Shale, slumping is very common, particularly in the "breaks" area. Deposits of slumped material and other gravity-caused detritus are called colluvium; however, the colluvium is so mixed with other waterlaid deposits that they are mapped together in figure 2. In the trench of the Missouri River, the Pierre Shale is absent and the underlying Niobrara Formation is exposed at the surface or lies beneath the flood-plain deposits.

Traces of ancient streams or rivers are preserved as alluvial deposits (Howells, 1974). A channel across the La Roche terrace (Clark Ranch) may be a former location of the Missouri River valley, a remnant of a preglacial drainage tributary to the Bad River (which enters the Missouri River about 15 miles to the northwest of the study area near Fort Pierre), or an ancient drainage valley of Medicine Creek. A portion of a buried valley, possibly deposits from an ancient channel of Medicine Creek, crosses the Grass Rope area. Such deposits typically contain shallow ground water and form fertile soils for farming.

\section{Bedrock Geology}

The Lower Brule Reservation lies on the northern slope of the Sioux uplift near its western end. The reservation extends from near the crest of the uplift down onto the shelf and the transition zone between the shelf and the Williston Basin to the northwest. The geologic units contained in the bedrock sequence, 


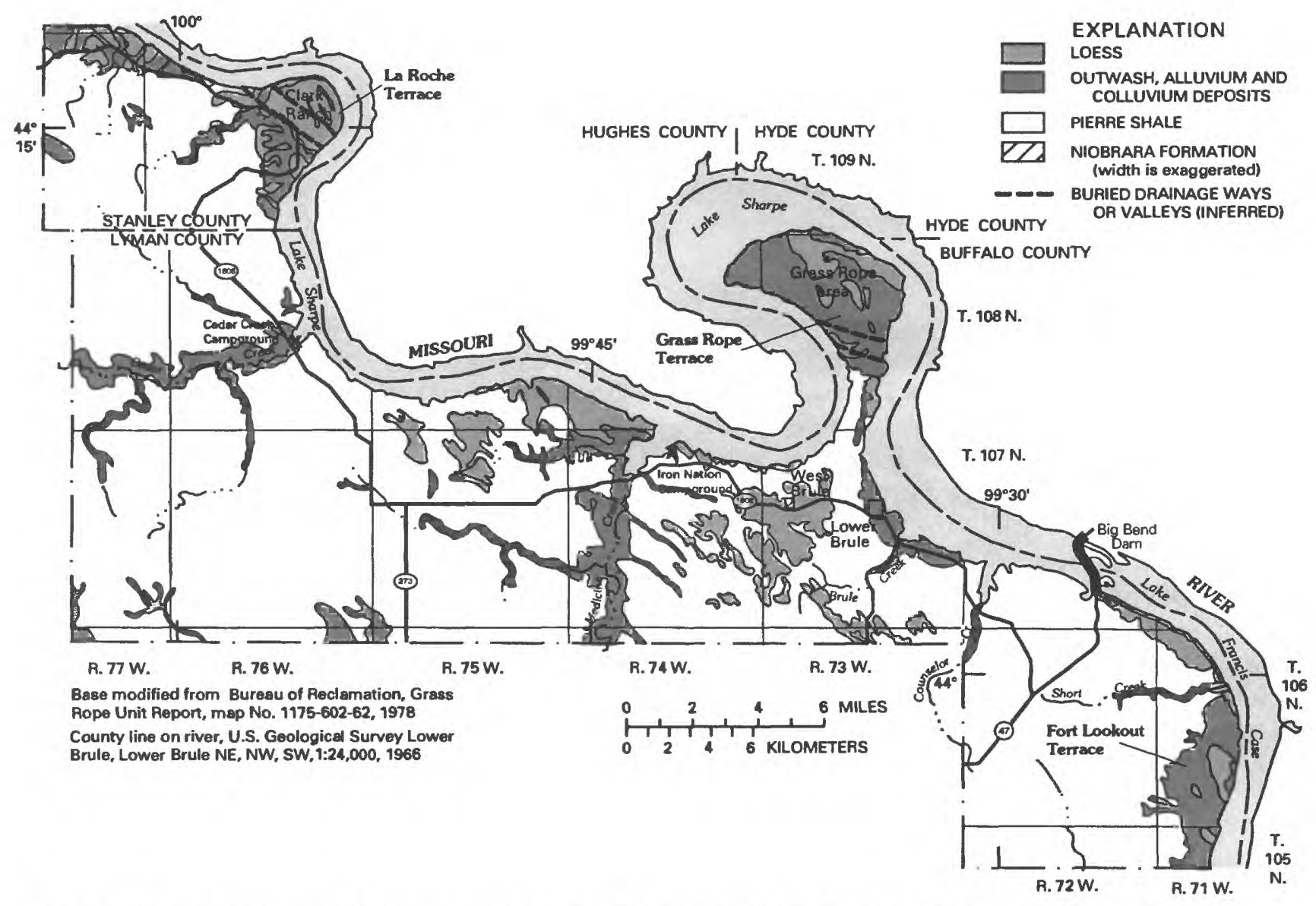

Figure 2. Generalized surficial geology on the Lower Brule Indian Reservation (modified from Howells, 1974).

and their hydrologic significance, are described in the generalized stratigraphic column (table 1).

\section{WATER RESOURCES}

Both surface water and ground water are important components of the water resources of the Lower Brule Reservation. However, surface water vastly overshadows ground water in terms of volume and potential for development.

\section{Surface-Water Availability and Quality}

The Missouri River is the major surface-water resource available to the reservation. Main-stem reservoirs adjacent to the reservation (Lakes Sharpe and Francis Case) provide a vast supply of good quality water.

\section{Rivers and Streams}

The Missouri River, as it enters the reservation, is regulated by three main-stem dams located upstream. The three dams, listed in upstream order, are: Oahe Dam, near Pierre, S. Dak.; Garrison Dam, near Bismarck, N. Dak.; and Fort Peck Dam, near Fort Peck, Mont. These upstream dams, which collectively store an average volume of 51.9 million acre-feet, regulate the year-round flow of the Missouri River and stabilize year-round variations in the quality of water in the river. Big Bend Dam, which forms Lake Sharpe, is adjacent to the Lower Brule Reservation. A fifth dam, Fort Randall Dam, is about 107 miles downstream from Big Bend Dam, but it backs water in its reservoir (Lake Francis Case) to Big Bend Dam. The storage capacities of the five reservoirs mentioned are listed in table 2.

A generalized hydrologic budget was developed for the reservation to quantify the overall surface- 
Table 1. Generalized stratigraphic chart of geologic units present in the Lower Brule Indian Reservation

[Modified from Howells (1974). --, not applicable]

\begin{tabular}{|c|c|c|c|c|}
\hline System & $\begin{array}{l}\text { Geologic } \\
\text { unit }\end{array}$ & $\begin{array}{l}\text { Thickness } \\
\text { (feet) }\end{array}$ & $\begin{array}{l}\text { General description and } \\
\text { hydrologic significance }\end{array}$ & Water quality \\
\hline \multirow[t]{8}{*}{ Cretaceous } & Pierre Shale & $0-550$ & $\begin{array}{l}\text { Dark-grey to black friable shale, marine; } \\
\text { relatively impermeable. }\end{array}$ & $\begin{array}{l}\text { Very saline, may contain selenium } \\
\text { above the drinking- water regulation } \\
\text { of the U.S. Environmental } \\
\text { Protection Agency (1993). }\end{array}$ \\
\hline & Niobrara Formation & $70-160$ & Chalky shale; relatively impermeable. & \\
\hline & Carlile Shale & $140-325$ & $\begin{array}{l}\text { Grey shale and silty sandstone; } \\
\text { relatively impermeable. }\end{array}$ & $\begin{array}{l}\text { Very saline, sodium chloride type } \\
\text { water. }\end{array}$ \\
\hline & Greenhorn Limestone & $50-100$ & $\begin{array}{l}\text { Blue to grey limestone and shale; can } \\
\text { yield muddy saline water in some } \\
\text { areas. }\end{array}$ & $\begin{array}{l}\text { Very muddy, sodium chloride type } \\
\text { water. }\end{array}$ \\
\hline & Graneros Shale & $150-320$ & $\begin{array}{l}\text { Dark-grey shale, marine; relatively } \\
\text { impermeable. May contain a sandy or } \\
\text { sandstone facies at its base in the } \\
\text { northeastern two-thirds of the } \\
\text { reservation. }\end{array}$ & $\begin{array}{l}\text { Muddy, saline, ranges from calcium } \\
\text { magnesium sulfate to sodium } \\
\text { chloride type water. Gassy. }\end{array}$ \\
\hline & $\begin{array}{l}\text { Dakota (Newcastle) } \\
\text { Sandstone }\end{array}$ & $280-350$ & $\begin{array}{l}\text { Sandstone and silty sandstone, deltaic or } \\
\text { near shore; major aquifer. }\end{array}$ & $\begin{array}{l}\text { Ranges from calcium magnesium } \\
\text { sulfate to sodium chloride type } \\
\text { water. Can be gassy in the upper } \\
\text { part of the formation. }\end{array}$ \\
\hline & Skull Creek Shale & $0-130$ & $\begin{array}{l}\text { Dark-grey shale; relatively } \\
\text { impermeable. }\end{array}$ & -- \\
\hline & Inyan Kara Group & $0-80$ & $\begin{array}{l}\text { Sandstone and siltstone, continental to } \\
\text { near shore; major aquifer. }\end{array}$ & $\begin{array}{l}\text { Calcium magnesium sulfate type } \\
\text { water. }\end{array}$ \\
\hline Jurassic & Sundance Formation & $0-90$ & $\begin{array}{l}\text { White to red sandstone and green and } \\
\text { grey shale, marine; major aquifer. }\end{array}$ & $\begin{array}{l}\text { Calcium magnesium sulfate type } \\
\text { water. }\end{array}$ \\
\hline $\begin{array}{l}\text { Permian and } \\
\text { Pennsylvanian }\end{array}$ & Minnelusa Formation & ${ }^{\mathrm{I}} 0-250$ & $\begin{array}{l}\text { Sandstone, dolomite, limestone, } \\
\text { anhydrite, and shale; continental to } \\
\text { marine; major aquifer. }\end{array}$ & $\begin{array}{l}\text { Calcium magnesium sulfate type } \\
\text { water. }\end{array}$ \\
\hline Mississippian & Madison Group & ${ }^{1} 0-100$ & $\begin{array}{l}\text { Limestone and dolomite and } \\
\text { interbedded shales and anhydrite; } \\
\text { major aquifer. }\end{array}$ & $\begin{array}{l}\text { Calcium magnesium sulfate type } \\
\text { water. }\end{array}$ \\
\hline Devonian & Undifferentiated & ${ }^{1} 0-50$ & $\begin{array}{l}\text { Shales interbedded with anhydrite, } \\
\text { sandstones and siltstones, and } \\
\text { limestones and dolomite, marine; } \\
\text { hydrologic potential unknown. }\end{array}$ & No data available. \\
\hline $\begin{array}{l}\text { Mississippian(?) } \\
\text { to Cretaceous(?) }\end{array}$ & $\begin{array}{l}\text { Transgressive facies } \\
\text { of clastic rocks; } \\
\text { locally called } \\
\text { "basal wash" }\end{array}$ & $0-50$ & $\begin{array}{l}\text { Primarily coarse-grained sandstone, } \\
\text { derived from underlying or nearby } \\
\text { Precambrian rocks; major aquifer. }\end{array}$ & $\begin{array}{l}\text { Calcium magnesium sulfate type } \\
\text { water. }\end{array}$ \\
\hline \multirow[t]{2}{*}{ Precambrian } & Sioux Quartzite & - & $\begin{array}{l}\text { Pink to purple orthoquartzite; } \\
\text { impermeable except where fractured. }\end{array}$ & $\begin{array}{l}\text { Calcium magnesium sulfate type } \\
\text { water. }\end{array}$ \\
\hline & $\begin{array}{l}\text { Igneous and } \\
\text { metamorphic rocks }\end{array}$ & -- & $\begin{array}{l}\text { Variety of granite, gneiss, schist, and } \\
\text { metamorphic rocks. Impermeable } \\
\text { except where fractured. }\end{array}$ & -- \\
\hline
\end{tabular}

\footnotetext{
${ }^{1}$ Maximum thickness unknown. Thickness given is speculative.
} 
Table 2. Storage capacities of Missouri River reservoirs adjacent to or upstream from the Lower Brule Indian Reservation [Source: U.S. Army Corps of Engineers (1993). --, not available]

\begin{tabular}{llccccc}
\hline \multirow{2}{*}{ Dam } & \multicolumn{1}{c}{ Reservoir } & Drainage area & \multicolumn{3}{c}{ Storage, in thousands of acre-feet } \\
\cline { 5 - 7 } & & & $\begin{array}{c}\text { Top of } \\
\text { (square miles) }\end{array}$ & $\begin{array}{c}\text { Top of flood } \\
\text { exclusive } \\
\text { control and } \\
\text { multiple use }\end{array}$ & $\begin{array}{c}\text { Top of } \\
\text { carryover } \\
\text { multiple use }\end{array}$ & $\begin{array}{c}\text { Top of } \\
\text { permanent } \\
\text { pool }\end{array}$ \\
\hline Fort Randall & Lake Francis Case & 263,480 & 5,494 & 4,508 & 3,193 & 1,545 \\
Big Bend & Lake Sharpe & 249,330 & 1,859 & 1,799 & - & 1,682 \\
Oahe & Lake Oahe & 243,490 & 23,137 & 22,035 & 18,834 & 5,373 \\
Garrison & Lake Sakakawea, N. Dak. & 181,400 & 23,821 & 22,332 & 18,110 & 4,980 \\
Fort Peck & Fort Peck Lake, Mont. & 57,500 & 18,688 & 17,713 & 14,996 & 4,211 \\
\hline
\end{tabular}

water system and to show the relative significance of the Missouri River as a water source (fig. 3). Because interaction between surface and ground water on the reservation is small and localized, the hydrologic budget as shown does not include the ground-water components. The budget is considered to represent the period 1968-92, although data for that period were not available for all components of the system.
The discharge of the Missouri River at Oahe Dam (located near Pierre) averaged 18,188,000 acre-feet during 1968-92 (table 3). Inflows to and diversions from the Missouri River between Oahe Dam and the Lower Brule Reservation were used to determine the Missouri River inflow to the reservation. The average inflow (rounded to $18,290,000$ acre-feet per year) was calculated by adding the discharge at Oahe Dam

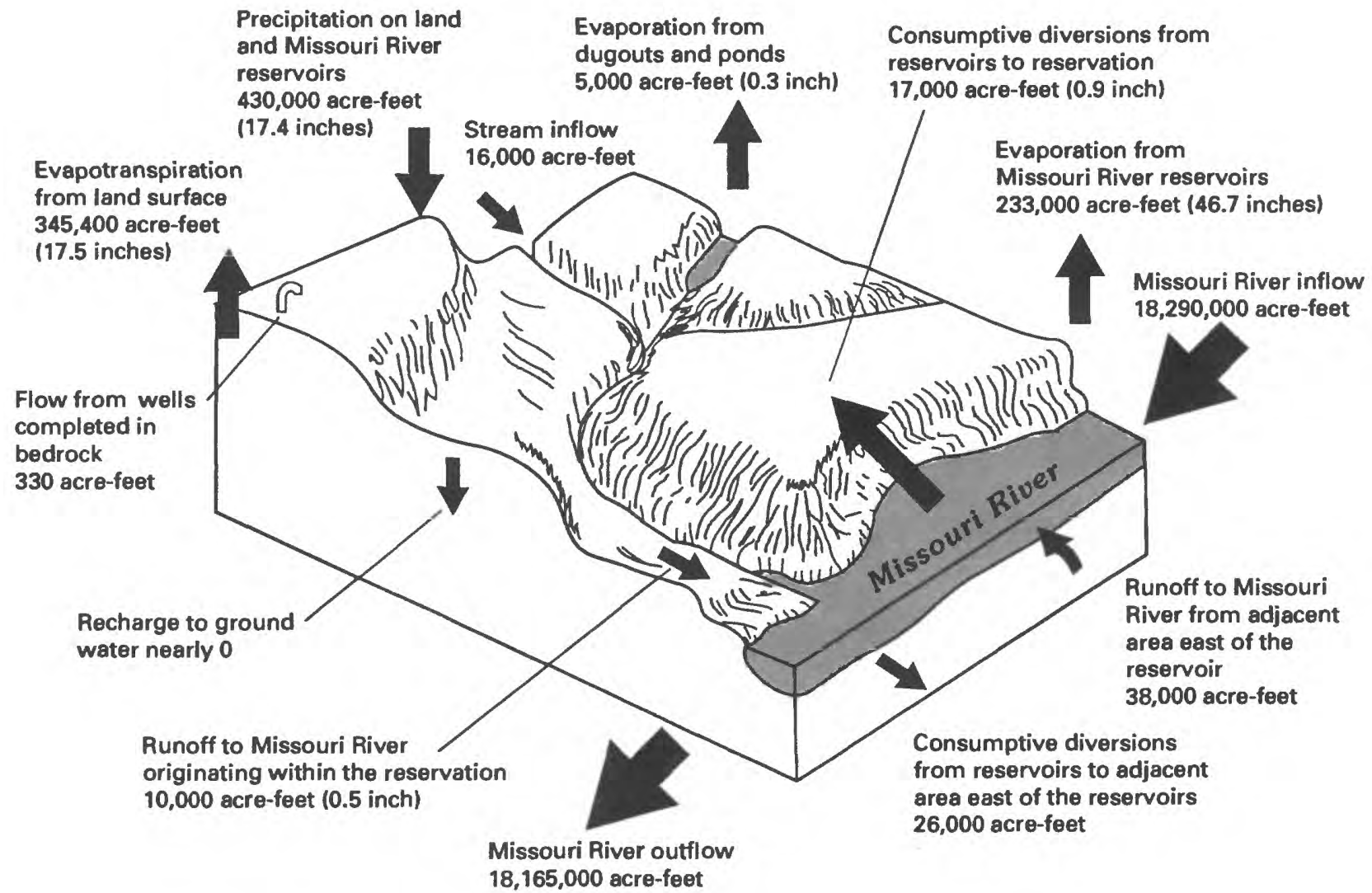

Figure 3. Generalized average annual hydrologic budget for the Lower Brule Indian Reservation. 
Table 3. Annual Missouri River discharge at Oahe and Big Bend Dams, 1968-92

\begin{tabular}{|c|c|c|}
\hline \multirow{2}{*}{ Year } & \multicolumn{2}{|c|}{ Discharge, in millions of acre-feet' } \\
\hline & Oahe Dam & Big Bend Dam \\
\hline 1968 & 17.8 & 17.6 \\
\hline 1969 & 20.3 & 20.2 \\
\hline 1970 & 21.4 & 21.0 \\
\hline 1971 & 25.2 & 25.1 \\
\hline 1972 & 23.7 & 23.9 \\
\hline 1973 & 15.2 & 15.1 \\
\hline 1974 & 18.1 & 17.1 \\
\hline 1975 & 26.6 & 26.6 \\
\hline 1976 & 22.5 & 22.4 \\
\hline 1977 & 16.5 & 16.8 \\
\hline 1978 & 21.6 & 22.1 \\
\hline 1979 & 19.5 & 19.7 \\
\hline 1980 & 18.6 & 18.4 \\
\hline 1981 & 17.7 & 17.1 \\
\hline 1982 & 17.3 & 16.9 \\
\hline 1983 & 16.8 & 16.6 \\
\hline 1984 & 17.5 & 18.4 \\
\hline 1985 & 16.4 & 16.2 \\
\hline 1986 & 17.1 & 18.5 \\
\hline 1987 & 16.1 & 16.9 \\
\hline 1988 & 16.6 & 16.6 \\
\hline 1989 & 15.2 & 14.9 \\
\hline 1990 & 13.1 & 12.7 \\
\hline 1991 & 12.4 & 12.7 \\
\hline 1992 & 11.5 & 11.6 \\
\hline Average & 18.2 & 18.2 \\
\hline
\end{tabular}

'Data supplied by Kevin Grode, U.S. Army Corps of Engineers, oral commun., March 1994.

(18,188,000 acre-feet) to inflows from the Bad River (90,750 acre-feet during 1968-92, calculated from U.S. Geological Survey (USGS) gaging-station records), inflows from Medicine Knoll Creek (4,360 acre-feet, calculated using Larimer's (1970) method), and inflows from Antelope Creek and a few ephemeral streams that drain land adjacent to the reservoir $(14,400$ acre-feet, calculated using Larimer's (1970) method), and by subtracting 1990 irrigation diversions (9,644 acre-feet, based on irrigation data stored in the USGS Water-Use Data System). Runoff to the Missouri River in the reach adjacent to the reservation was determined to be 26,000 acre-feet per year from the west side (the part of the reservation in Lyman and Stanley Counties) and 38,000 acre-feet per year from the east side (Hughes, Hyde, and Buffalo Counties) (Larimer, 1970). Diversions from the reach of the Missouri River adjacent to the reservation were virtually all for irrigation use and were determined to be 17,000 acre-feet per year to the west side and 26,000 acre-feet per year to the east side (based on 1990 usage from the USGS Water-Use Data System). Precipitation falling on the reservoir surfaces was estimated to be about 87,000 acre-feet per year, which was calculated by multiplying 17.4 inches of precipitation (determined using a "polygon-of-influence" method and 1951-80 normal annual precipitation for local weather stations) times 59,900 surface acres. Evaporation from the reservoir surfaces was calculated to be about 233,000 acre-feet per year, which was calculated by multiplying 46.7 inches of freewater-surface evaporation (Farnsworth and others, 1982 ) times 59,900 acres. Using the $18,290,000$ acrefeet of Missouri River inflow to the reservation, adding inflows of 64,000 acre-feet, subtracting diversions of 43,000 acre-feet, adding precipitation $(87,000$ acre-feet), and subtracting evaporation (233,000 acre-feet), the average outflow of the Missouri River from the Lower Brule Reservation was calculated to be $18,165,000$ acre-feet per year.

Precipitation falling on the land surface of the reservation was calculated to be about 343,070 acrefeet per year (17.4 inches falling on 236,600 acres). Using this value for precipitation in conjunction with runoff originating within the reservation $(10,000$ acrefeet per year), diversions from the Missouri River to the reservation that are consumptively used (17,000 acre-feet per year), flow from wells completed in bedrock (330 acre-feet per year, determined during USGS well inventory), and evaporation from ponds and dugouts (5,000 acre-feet per year), evapotranspiration from the land surface of the reservation was calculated to be 345,400 acre-feet per year (17.5 inches).

The USGS operated continuous streamflow-gaging stations on the Missouri River at Pierre (station 
06440000) during water years 1934-65 and on the Missouri River at Chamberlain (station 06443000) from September 1928 to September 1929 and from April 1945 to September 1954. Daily values for these two stations are presented in tables 15 and 16 in the Supplemental Information section at the end of this report.

Compared to the annual flow of the Missouri River at Big Bend Dam and the storage of the reservoirs, the amount of surface water used for public water supply and irrigation on the reservation is negligible (less than one-tenth of one percent). Within the boundaries of the reservation, 146 to 157 acre-feet per year is used for public supplies at the towns of Lower Brule and West Brule (Scott Jones, Lower Brule Sioux Tribe, oral commun., Aug. 30, 1993). Irrigation use by the Lower Brule Tribe in the Clark Ranch and Grass Rope areas (fig. 1) was 2,629 and 8,075 acrefeet, respectively, in 1990. Nontribal irrigation use on the reservation in 1990 was 6,414 acre-feet. The reservoirs, particularly Lake Sharpe and the tailwater of Big Bend Dam on Lake Francis Case that normally store about 5 million acre-feet of water, also provide recreational opportunities such as fishing, boating, waterfowl hunting, and camping along their shorelines.

The Missouri is the only river or stream within the reservation boundaries that flows continuously throughout the year. Most local streamflow reaching the Missouri River is from snowmelt and spring rainfall. Runoff from all but the most intense summer storms usually infiltrates into the subsurface or evaporates before it reaches the Missouri River.

The estimated average annual streamflow and peak flows for selected streams on the Lower Brule Reservation are shown in table 4 . The peak-flow data in table 4 are statistical calculations of the magnitudes of peaks that could be expected to be equaled or exceeded an average of every $2,5,10,25,50$, and 100 years.

The peak flows for Cedar Creek on the Lower Brule Reservation were estimated using techniques developed by Becker (1974), which use drainage area (164 square miles) and mean basin elevation ( 1,837 feet). The peak flows for Medicine Creek were estimated by taking the square root of the ratio of the drainage area of Medicine Creek at the mouth

Table 4. Estimated average annual streamflow and peak flows for given recurrence intervals for selected streams on the Lower Brule Indian Reservation

$\left[\mathrm{mi}^{2}\right.$, square mile; $\mathrm{ft}^{3} / \mathrm{s}$, cubic foot per second; --, not calculated]

\begin{tabular}{|c|c|c|c|c|c|c|c|c|}
\hline \multirow{2}{*}{ Drainage basin } & \multirow{2}{*}{$\begin{array}{l}\text { Drainage } \\
\text { area } \\
\left(\mathbf{m i}^{2}\right)\end{array}$} & \multirow{2}{*}{$\begin{array}{c}\text { Average } \\
\text { annual } \\
\text { streamflow } \\
\left(\mathrm{ft}^{3} / \mathrm{s}\right)\end{array}$} & \multicolumn{6}{|c|}{$\begin{array}{l}\text { Peak flow }{ }^{2.3,4} \text {, in cubic feet per second, } \\
\text { for the given recurrence interval, in years }\end{array}$} \\
\hline & & & 2 & 5 & 10 & 25 & 50 & 100 \\
\hline Cedar Creek & 164 & 4.7 & 853 & 2,340 & 3,780 & 6,780 & 10,300 & 13,200 \\
\hline $\begin{array}{l}\text { Cedar Creek flow } \\
\text { originating within the } \\
\text { reservation }\end{array}$ & 81.5 & 2.3 & - & - & -- & -- & - & -- \\
\hline Medicine Creek & 689 & 19.9 & 789 & 3,690 & 7,700 & 16,100 & 25,000 & 36,700 \\
\hline $\begin{array}{l}\text { Medicine Creek flow } \\
\text { originating within the } \\
\text { reservation }\end{array}$ & 80.5 & 2.4 & - & -- & -- & -- & - & -- \\
\hline Brule Creek & 12.7 & .4 & 100 & 280 & 500 & 910 & 1,400 & 1,900 \\
\hline Counselor Creek & 29.3 & .9 & 130 & 380 & 690 & 1,300 & 2,000 & 2,800 \\
\hline
\end{tabular}

${ }^{1}$ From Howells (1974). Estimated using the method described by Larimer (1970).

${ }^{2}$ Based on technique developed by Becker (1974) for Cedar Creek.

${ }^{3}$ Based on Log-Pearson Type III analysis for period of record 1955-91 for Medicine Creek.

${ }^{4}$ Based on technique developed by Becker (1980) for Brule Creek and Counselor Creek. 
(689 square miles) to the drainage area at the streamflow-gaging station on Medicine Creek at Kennebec (station 06442500, located upstream from the reservation) (465 square miles) times the respective peak flows calculated for the Medicine Creek gaging station. The peak flows at the Medicine Creek gaging station were determined by using LogPearson Type III analysis for the period of record (1955-91).

The peak flows for Brule Creek and Counselor Creek were estimated using techniques developed by Becker (1980). The equations for peak flows are based on regression relations that are considered applicable (contributing drainage area, main channel slope, and a soil-infiltration index) for estimating flood peaks at sites on basins that have less than 100 square miles of drainage area.

The system of large reservoirs on the Missouri River has stabilized the quality of the river water entering the reservation to a year-round average of slightly less than $500 \mathrm{mg} / \mathrm{L}$ of dissolved solids. The water can be characterized as a calcium sodium bicarbonate sulfate type water. The process of water- quality stabilization began in 1937 with the closure of Fort Peck Dam in Montana, continued with the closures of Garrison Dam in North Dakota in 1953 and Oahe Dam near Pierre, South Dakota, in 1958, and reached its present level in 1964 with the filling of Lake Sharpe, created by the closure of Big Bend Dam in 1963. A summary of chemical analyses of Missouri River water collected at station 06440000 at Pierre before filling of Lake Oahe is given in table 5, and after filling of Lake Oahe is given in table 6. It should be noted that trace-element data presented in this report were not collected or processed using ultraclean procedures and values could reflect contamination introduced during some phase of collection and processing.

U.S. Environmental Protection Agency (EPA) drinking-water regulations are listed in table 7 for comparison with the water-quality data in tables 5 and 6, as well as other water-quality data presented later in the report. The excellent quality of water in the Missouri River at Pierre after filling of Lake Oahe is evident from comparison of the data in table 6 with the drinking-water regulations in table 7.

Table 5. Summary of chemical analyses of water from the Missouri River at Pierre (station 06440000) before filling of Lake Oahe

[Samples were collected between October 1, 1953, and September 30, 1958. All analyses by U.S. Geological Survey. Reported in milligrams per liter except as indicated. ${ }^{\circ} \mathrm{C}$, degree Celsius; $\mu \mathrm{S} / \mathrm{cm}$, microsiemens per centimeter at $25^{\circ} \mathrm{C}$; - , not determined]

\begin{tabular}{lccccc}
\hline \multicolumn{1}{c}{ Parameter } & $\begin{array}{c}\text { Number } \\
\text { of samples }\end{array}$ & Minimum & Maximum & Mean & Median \\
\hline Specific conductance $(\mu \mathrm{S} / \mathrm{cm})$ & 78 & 469 & 997 & 725 & 718 \\
$\mathrm{pH}$, field (standard units) & 79 & 7.0 & 8.3 & -- & 7.8 \\
Hardness, total (as $\mathrm{CaCO}_{3}$ ) & 79 & 145 & 354 & 232 & 228 \\
Dissolved solids, residue on evaporation at $180^{\circ} \mathrm{C}$ & 79 & 307 & 872 & 487 & 476 \\
Calcium, dissolved & 16 & 46 & 75 & 56 & 56 \\
Magnesium, dissolved & 16 & 16 & 26 & 20 & 20 \\
Sodium, dissolved & 79 & 41 & 124 & 68 & 66 \\
Sodium, percent & 79 & 34 & 44 & 39 & 38 \\
Sodium-adsorption ratio (units) & 79 & 1.4 & 2.9 & 1.9 & 1.9 \\
Potassium, dissolved & 16 & 3.2 & 7.0 & 4.7 & 4.6 \\
Sulfate, dissolved & 48 & 113 & 290 & 208 & 208 \\
Chloride, dissolved & 23 & 5.5 & 12.0 & 8.8 & 8.7 \\
Fluoride, dissolved & 16 & 0.4 & 0.7 & 0.5 & 0.5 \\
Silica, dissolved & 16 & 7.9 & 20 & 12 & 11 \\
\hline
\end{tabular}


Table 6. Summary of chemical, biological, and suspended-sediment analyses of water from the Missouri River at Pierre (station 06440000) after filling of Lake Oahe

[Samples collected between July 1, 1971, and August 19, 1986. All analyses by U.S. Geological Survey. Reported in milligrams per liter except as indicated. $\mu \mathrm{S} / \mathrm{cm}$, microsiemens per centimeter at $25^{\circ} \mathrm{C}$; NTU, nephelometric turbidity units; $\mu \mathrm{g} /$, microgram per liter; col/100 mL, colonies per $100 \mathrm{milliliters;} \mathrm{mm}$, millimeter; K, Results based on colony count outside the acceptance range (nonideal colony count); --, not determined; $<$, less than]

\begin{tabular}{|c|c|c|c|c|c|}
\hline Parameter & $\begin{array}{l}\text { Number of } \\
\text { samples }\end{array}$ & Minimum & Maximum & Mean $^{1}$ & Median $^{1}$ \\
\hline Specific conductance $(\mu \mathrm{S} / \mathrm{cm})$ & 199 & 600 & 910 & 744 & 736 \\
\hline pH, field, (standard units) & 199 & 7.4 & 10.2 & -- & 8.2 \\
\hline Turbidity (NTU) & 68 & 0.1 & 26 & 3.3 & 2.0 \\
\hline Oxygen, dissolved & 106 & 6.6 & 17.4 & 10.5 & 9.9 \\
\hline Oxygen, dissolved (percent of saturation) & 19 & 86 & 108 & 97 & 96 \\
\hline Hardness, total (as $\mathrm{CaCO}_{3}$ ) & 179 & 200 & 270 & 230 & 230 \\
\hline Alkalinity, total (as $\mathrm{CaCO}_{3}$ ) & 152 & 126 & 190 & 157 & 157 \\
\hline Dissolved solids, residue on evaporation at $180^{\circ} \mathrm{C}$, dissolved & 122 & 396 & 617 & 495 & 491 \\
\hline Calcium, dissolved & 179 & 49 & 65 & 57 & 57 \\
\hline Magnesium, dissolved & 179 & 18 & 27 & 22 & 22 \\
\hline Sodium, dissolved & 179 & 56 & 89 & 69 & 68 \\
\hline Sodium, percent & 179 & 34 & 43 & 38 & 38 \\
\hline Sodium-adsorption ratio (units) & 179 & 2 & 2 & 2 & 2 \\
\hline Potassium, dissolved & 179 & 3.8 & 12 & 4.8 & 4.7 \\
\hline Sulfate, dissolved & 178 & 170 & 280 & 210 & 210 \\
\hline Chloride, dissolved & 179 & 7.6 & 17 & 10 & 10 \\
\hline Fluoride, dissolved & 176 & 0.3 & 0.9 & 0.5 & 0.5 \\
\hline Silica, dissolved & 176 & 2.4 & 9.7 & 6.2 & 6.2 \\
\hline Nitrogen, nitrite plus nitrate, dissolved (as $\mathrm{N}$ ) & 134 & $<0.1$ & 5.0 & 0.20 & 0.15 \\
\hline Nitrogen, ammonia, dissolved (as $\mathrm{N}$ ) & 58 & $<0.1$ & 0.25 & 0.06 & 0.04 \\
\hline Nitrogen, ammonia plus organic, total (as $\mathrm{N}$ ) & 117 & 0.05 & 4.2 & 0.5 & 0.3 \\
\hline Phosphorus, total (as P) & 177 & $<0.01$ & 3.8 & 0.06 & 0.02 \\
\hline Phosphorus, dissolved (as P) & 133 & $<0.01$ & 1.5 & 0.03 & 0.01 \\
\hline Phosphorus, orthophosphate, dissolved (as P) & 105 & $<0.01$ & 1.2 & 0.02 & 0.01 \\
\hline Arsenic, dissolved $(\mu \mathrm{g} / \mathrm{L})$ & 47 & 1 & 3 & 2 & 2 \\
\hline Barium, dissolved $(\mu \mathrm{g} / \mathrm{L})$ & 36 & 36 & 200 & 55 & 46 \\
\hline Beryllium, dissolved $(\mu \mathrm{g} / \mathrm{L})$ & 16 & $<0.5$ & 2 & 0.5 & 0.4 \\
\hline Cadmium, dissolved $(\mu \mathrm{g} / \mathrm{L})$ & 47 & $<1$ & 8 & 1 & 0.4 \\
\hline Copper, dissolved $(\mu \mathrm{g} / \mathrm{L})$ & 47 & $<1$ & 17 & 4 & 3 \\
\hline Iron, dissolved $(\mu \mathrm{g} / \mathrm{L})$ & 47 & 3 & 300 & 25 & 9 \\
\hline Lead, dissolved $(\mu \mathrm{g} / \mathrm{L})$ & 47 & $<1$ & 21 & 3 & 2 \\
\hline Lithium, dissolved $(\mu \mathrm{g} / \mathrm{L})$ & 16 & 49 & 67 & 55 & 54 \\
\hline Manganese, dissolved $(\mu \mathrm{g} / \mathrm{L})$ & 47 & $<1$ & 30 & 8 & 7 \\
\hline Mercury, dissolved $(\mu \mathrm{g} / \mathrm{L})$ & 47 & $<0.1$ & 0.5 & 0.1 & 0.1 \\
\hline Selenium, dissolved $(\mu \mathrm{g} / \mathrm{L})$ & 48 & $<1$ & 2 & 1 & 1 \\
\hline Strontium, dissolved $(\mu \mathrm{g} / \mathrm{L})$ & 16 & 540 & 600 & 570 & 560 \\
\hline Fecal coliform ${ }^{2}(\mathrm{col} / 100 \mathrm{~mL})$ & 84 & $<1$ & K140 & 12 & 2 \\
\hline Fecal streptococci ${ }^{2}(\mathrm{col} / 100 \mathrm{~mL})$ & 86 & $<1$ & K290 & 30 & 12 \\
\hline Sediment, suspended concentration & 82 & 2 & 162 & 45 & 41 \\
\hline Sediment, suspended, sieve diameter, percent finer than $0.062 \mathrm{~mm}$ & 57 & 9.4 & 100 & 83 & 92 \\
\hline
\end{tabular}

\footnotetext{
${ }^{1} \mathrm{~A}$ log probability regression method (Helsel and Gilliom, 1985) was used to estimate means and medians for constituents that had analytical concentrations below the reporting limit.

${ }^{2}$ Summary statistics for bacterial parameters were calculated using nonideal colony counts.
} 
Table 7. U.S. Environmental Protection Agency drinkingwater regulations

[Contaminants regulated under the Safe Drinking Water Act of 1986. --, no established regulation; NTU, nephelometric turbidity units; $\mathrm{mg} / \mathrm{L}$, milligram per liter; $\mu \mathrm{g} / \mathrm{L}$, microgram per liter]

\begin{tabular}{|c|c|c|}
\hline & $\begin{array}{l}\text { Primary } \\
\text { drinking-water } \\
\text { regulation }\end{array}$ & $\begin{array}{l}\text { Secondary } \\
\text { drinking-water } \\
\text { regulation }^{1}\end{array}$ \\
\hline & $\mathrm{MCL}^{2}$ & $\mathrm{SMCL}^{3}$ \\
\hline $\mathrm{pH}$ (standard units) & -- & $6.5-8.5$ \\
\hline Turbidity (NTU) & $<5$ & -- \\
\hline Total dissolved solids (mg/L) & - & 500 \\
\hline Sulfate $(\mathrm{mg} / \mathrm{L})$ & -- & 250 \\
\hline Chloride (mg/L) & - & 250 \\
\hline Fluoride (mg/L) & 4.0 & 2.0 \\
\hline Nitrate, as N (mg/L) & 10 & - \\
\hline Nitrate + nitrite, as $\mathrm{N}(\mathrm{mg} / \mathrm{L})$ & 10 & - \\
\hline Arsenic $(\mu \mathrm{g} / \mathrm{L})$ & 50 & - \\
\hline Barium $(\mu \mathrm{g} / \mathrm{L})$ & 2,000 & - \\
\hline Beryllium $(\mu \mathrm{g} / \mathrm{L})$ & 4 & - \\
\hline Cadmium $(\mu \mathrm{g} / \mathrm{L})$ & 5 & - \\
\hline Copper $(\mu \mathrm{g} / \mathrm{L})$ & -- & 1,000 \\
\hline Iron $(\mu \mathrm{g} / \mathrm{L})$ & - & 300 \\
\hline Lead $(\mu \mathrm{g} / \mathrm{L})$ & 50 & - \\
\hline Manganese $(\mu \mathrm{g} / \mathrm{L})$ & -- & 50 \\
\hline Mercury $(\mu \mathrm{g} / \mathrm{L})$ & 2 & - \\
\hline Selenium $(\mu \mathrm{g} / \mathrm{L})$ & 50 & -- \\
\hline
\end{tabular}

${ }^{1}$ U.S. Environmental Protection Agency (1993).

${ }^{2} \mathrm{MCL}=$ Maximum Contaminant Level: Enforceable, health-based regulation.

${ }^{3} \mathrm{SMCL}=$ Secondary Maximum Contaminant Level: Contaminants that affect the esthetic quality of drinking water. At high concentrations or values, health implications as well as esthetic degradation may exist. SMCL's are not federally enforceable but are intended as guidelines for the States.

Little water-quality data are available for streams on the reservation. The USGS water-quality data base contains only one analysis each for Brule Creek and Medicine Creek. These data are presented in table 8. The 1971 samples exceeded EPA drinking-water regulations (U.S. Environmental Protection Agency, 1993) for chloride, sulfate, and dissolved solids in Brule Creek and for sulfate, cadmium, and dissolved solids in Medicine Creek.

It is not possible to characterize the quality of water in Brule and Medicine Creeks based on only one sample taken more than 20 years ago. However, specific-conductance measurements for Medicine Creek at Kennebec may provide some information on general water-quality variability for ephemeral tributaries within the reservation. Fifty specific-conductance measurements made by the USGS at this site during the period 1978-92 ranged from 284 to $2,500 \mu \mathrm{S} / \mathrm{cm}$, with a median of $762 \mu \mathrm{S} / \mathrm{cm}$. Specific conductance generally was lower during larger flows and higher as flow approached zero. Generally, major-ion chemistry of ephemeral tributaries on the reservation would be expected to vary widely from year to year and during individual years, depending largely on flow conditions.

\section{Stock Ponds and Dugouts}

Except for the terraces and parts of the rolling uplands on the Lower Brule Reservation, much of the land is unsuitable for large-scale crop production because of rugged topography and/or poor soils. The Bureau of Indian Affairs (1964) soil and range studies revealed that only 16 percent of the land on the Lower Brule Reservation is suitable for production of cultivated crops. Thus, much of the area is rangeland or hayland used for ranching. For many ranchers, the least expensive way to provide dispersed water supplies is to capture and hold spring runoff and occasional summer stormflow in stock ponds (formed by a dam) or dugouts constructed in ephemeral streambeds and drainageways.

Several surveys have been made to inventory the stock ponds and dugouts on the Lower Brule Reservation. A survey conducted by Howells (1974) indicated that there were 526 stock ponds and dugouts on the reservation in 1970 . Of these, 343 (65 percent) were less than 1 acre in size; 158 ( 30 percent) were between 1 acre and 5 acres in size; and 25 ( 5 percent) were greater than 5 acres but less than 20 acres in size.

In a 1979 stock-dam inventory of the reservation made by the Bureau of Indian Affairs (Division of Natural Resources, written commun., undated), 160 stock ponds and dugouts were located and mapped, and 109 were measured for depth and size. The condition of each pond or dugout was ranked qualitatively as shown in table 9. The survey indicated that in most shallow ponds (less than 8 feet deep), the 
Table 8. Chemical analyses of water samples collected from Brule and Medicine Creeks near Lower Brule, April 1, 1971

[Analyses by U.S. Geological Survey. Reported in milligrams per liter (mg/L) except as indicated. ${ }^{\circ} \mathrm{C}$. degree Celsius; $\mu \mathrm{S} / \mathrm{crn}$, microsiemens per centimeter at $25^{\circ} \mathrm{C} ; \mu \mathrm{g} /$, micrograms per liter; ND, analyzed for but not detected; --, no data]

\begin{tabular}{|c|c|c|c|c|c|}
\hline Parameter & $\begin{array}{l}\text { Brule Creek near } \\
\text { Lower Brule } \\
\text { (Site ID } \\
440334099340500)^{1}\end{array}$ & $\begin{array}{c}\text { Medicine Creek } \\
\text { near Lower Brule } \\
\text { (Site ID } \\
\mathbf{4 4 0 3 5 7 0 9 9 4 4 1 1 0 0 )}^{1}\end{array}$ & Parameter & $\begin{array}{c}\text { Brule Creek near } \\
\text { Lower Brule } \\
\text { (Site ID } \\
440334099340500)^{1}\end{array}$ & $\begin{array}{c}\text { Medicine Creek } \\
\text { near Lower Brule } \\
\text { (Site ID } \\
\text { 440357099441100) }\end{array}$ \\
\hline \multirow{2}{*}{$\begin{array}{l}\text { Specific conductance } \\
(\mu \mathrm{S} / \mathrm{cm})\end{array}$} & \multirow[t]{2}{*}{3,470} & \multirow[t]{2}{*}{1,090} & Bicarbonate, field & 300 & 130 \\
\hline & & & Carbonate, field & 0 & 0 \\
\hline pH (standard units) & 8.1 & 7.9 & Sulfate, dissolved & 1,400 & 440 \\
\hline \multirow{2}{*}{$\begin{array}{l}\text { Hardness, total (as } \\
\mathrm{CaCO}_{3} \text { ) }\end{array}$} & \multirow[t]{2}{*}{1,100} & \multirow{2}{*}{390} & Chloride, dissolved & 300 & 28 \\
\hline & & & Fluoride, dissolved & 1.0 & 0.50 \\
\hline \multirow{2}{*}{$\begin{array}{l}\text { Hardness, } \\
\text { noncarbonate (as } \\
\mathrm{CaCO}_{3} \text { ) }\end{array}$} & \multirow[t]{2}{*}{810} & \multirow[t]{2}{*}{270} & Silica, dissolved & 17 & 12 \\
\hline & & & $\begin{array}{l}\text { Nitrogen, nitrate, } \\
\text { dissolved (as N) }\end{array}$ & 0.22 & \multirow[b]{2}{*}{0.40} \\
\hline $\begin{array}{l}\text { Alkalinity, field (as } \\
\mathrm{CaCO}_{3} \text { ) }\end{array}$ & 248 & 109 & $\begin{array}{l}\text { dissolved (as } \mathrm{N} \text { ) } \\
\text { Nitrogen, nitrate, } \\
\text { dissolved (as } \mathrm{NO}_{3} \text { ) }\end{array}$ & 1.0 & \\
\hline $\begin{array}{l}\text { Solids, dissolved, sum } \\
\text { of constituents }\end{array}$ & 2,700 & 781 & $\begin{array}{l}\text { dissolved (as } \mathrm{NO}_{3} \text { ) } \\
\text { Phosphorus, total (as P) }\end{array}$ & 0.07 & 0.09 \\
\hline $\begin{array}{l}\text { Solids, dissolved, } \\
\text { residue at } 180^{\circ} \mathrm{C}\end{array}$ & 2,890 & 825 & $\begin{array}{l}\text { Arsenic, dissolved } \\
(\mu \mathrm{g} / \mathrm{L})\end{array}$ & ND & ND \\
\hline $\begin{array}{l}\text { Solids, dissolved (tons } \\
\text { per day) }\end{array}$ & 0.55 & - & $\begin{array}{l}\text { Boron, dissolved } \\
(\mu \mathrm{g} / \mathrm{L})\end{array}$ & 780 & 110 \\
\hline $\begin{array}{l}\text { Solids, dissolved (tons } \\
\text { per acre-foot) }\end{array}$ & 3.93 & 1.12 & $\begin{array}{l}\text { Cadmium, dissolved } \\
(\mu \mathrm{g} / \mathrm{L})\end{array}$ & 2.0 & 12 \\
\hline Calcium, dissolved & 280 & 110 & Lithium, dissolved & 210 & 90 \\
\hline Magnesium, dissolved & 85 & 27 & & & \\
\hline Sodium, dissolved & 440 & 87 & $\begin{array}{l}\text { Mercury, dissolved } \\
(\mu \mathrm{g} / \mathrm{L})\end{array}$ & ND & ND \\
\hline Sodium, percent & 47 & 32 & Selenium, dissolved & ND & ND \\
\hline Sodium adsorption & 6 & 2 & $(\mu \mathrm{g} / \mathrm{L})$ & & \\
\hline $\begin{array}{l}\text { ratio } \\
\text { Potassium, dissolved }\end{array}$ & 17 & 9.5 & $\begin{array}{l}\text { Strontium, dissolved } \\
(\mu \mathrm{g} / \mathrm{L})\end{array}$ & 5,800 & 1,100 \\
\hline
\end{tabular}

${ }^{1}$ The site-numbering system of the U.S. Geological Survey is based on the international system of latitude and longitude. The number contains 15 digits. The first six digits denote the degrees, minutes, and seconds of latitude north of the equator. The next seven digits denote degrees, minutes, and seconds of longitude west of the prime (Greenwich) meridian. The last two digits are sequential numbers within the same 1-second block. These systems provide the geographic location of the site and a unique number for each site.

storage capacity had been reduced because of siltation. Many of the dams were in suitable locations to capture surface runoff; however, their effectiveness was reduced because they were old, seepage through the dams was substantial, and the spillways were in poor condition.

For this study, an inventory of ponds was made from aerial photographs taken in August and September 1991; there were 593 stock ponds and dugouts (which had an estimated total area of 1,300 acres) and 29 other ponds (sewage ponds, sewage lagoon, and irrigation seepage or drainage ponds) within the reservation boundary. Of the 593 stock ponds and dugouts, 284 were less than 1 acre in size; 268 were from 1 to 5 acres in size; 34 were from 5 to 20 acres in size; and 4 were greater than 20 acres in size (size was not determined for three ponds).

During February of 1993, 52 stock ponds and dugouts were visited (fig. 4). The condition of all but two of the ponds that were visited was ranked qualitatively according to the system used for the 1979 survey. A tabulation of the ponds and dugouts visited and comments on their condition are included in table 17 in the Supplemental Information section. Of the 50 sites that were ranked, 33 were ranked as poor, 5 below average, and 12 average or better. 
Table 9.--Qualitative ranking of stock ponds and dugouts on the Lower Brule Indian Reservation used in the 1979 Bureau of Indian Affairs (Division of Natural Resources, written commun., undated) inventory

\begin{tabular}{|c|c|c|}
\hline Ranking criteria & $\begin{array}{c}\text { Qualitative } \\
\text { rank }\end{array}$ & $\begin{array}{l}\text { Number of } \\
\text { stock ponds } \\
\text { and dugouts }\end{array}$ \\
\hline $\begin{array}{l}\text { When full, depth is equal to or } \\
\text { greater than } 10 \text { feet and } \\
\text { surface area is greater than or } \\
\text { equal to } 2 \text { acres. }\end{array}$ & Average or better & 4 \\
\hline $\begin{array}{l}\text { When full, depth equals } 10 \text { feet } \\
\text { and surface area is less than } \\
2 \text { acres; or pond depth is } \\
\text { equal to or greater than } 6 \text { feet } \\
\text { and less than } 10 \text { feet, any } \\
\text { surface area. }\end{array}$ & Below average & 49 \\
\hline $\begin{array}{l}\text { When full, depth is less than } \\
6 \text { feet (silted in), any surface } \\
\text { area. }\end{array}$ & Poor & 56 \\
\hline
\end{tabular}

The locations of those stock ponds and dugouts in apparently usable condition that were inventoried from 1991 aerial photographs, but not visited, are shown in figure 5. These ponds also are listed by location of the dam or dugout, along with estimated size, in table 17. The locations of the 62 stock dams that were breached or possibly breached, as observed either by visit or on aerial photographs, are shown in figure 6. Information about the ponds formerly impounded by these dams also is given in table 17 .

The chemical quality of water in stock ponds and dugouts varies, depending upon the water source and time of the year. Water quality probably is best in spring following snowmelt and spring rains and probably is poorest in late summer, fall, or winter when water in ponds has been concentrated by evapotranspiration and/or ice formation and may have been stagnant for several months.

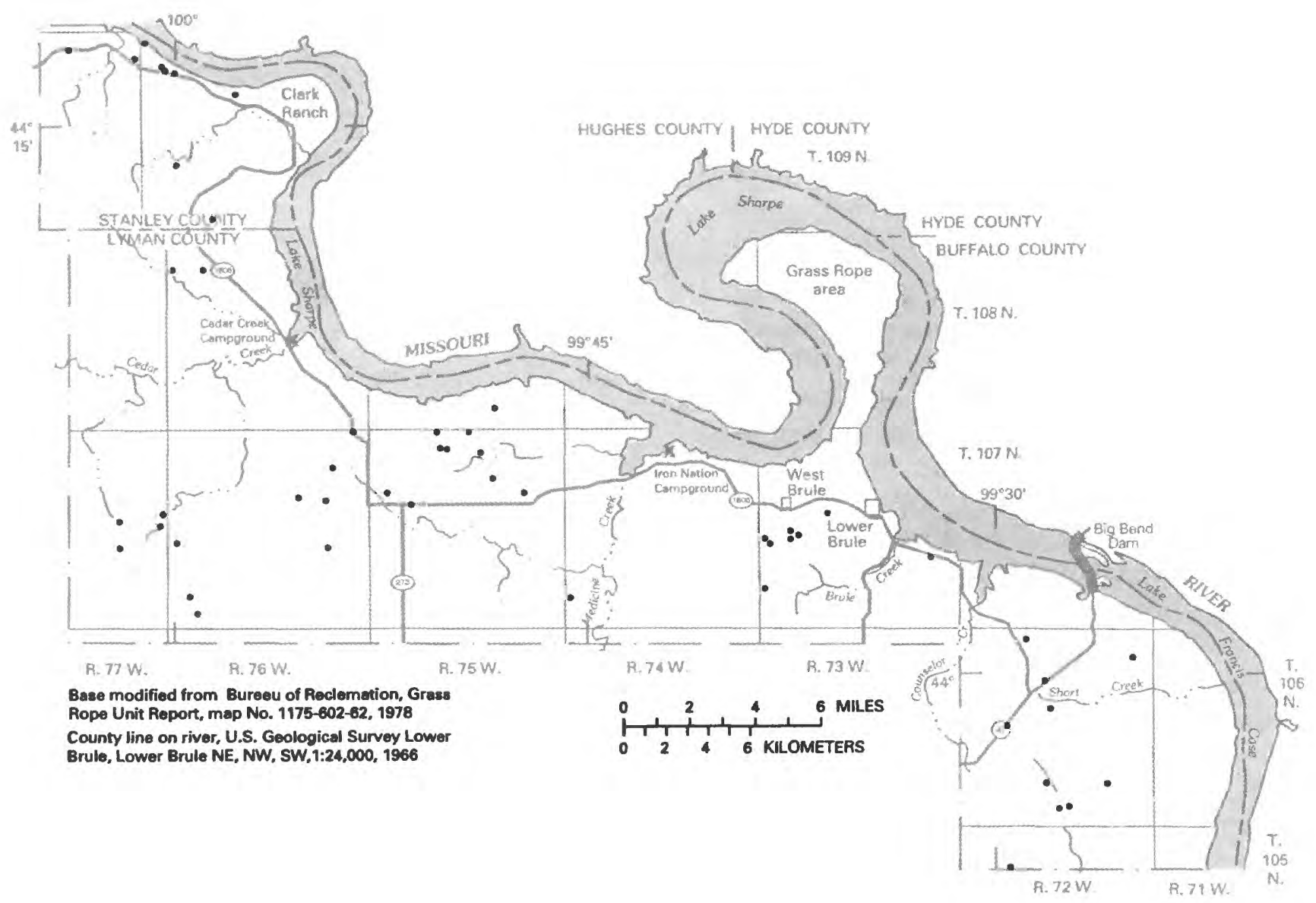

Flgure 4. Locations of stock ponds and dugouts on the Lower Brule Indian Reservation visited in February 1993. 


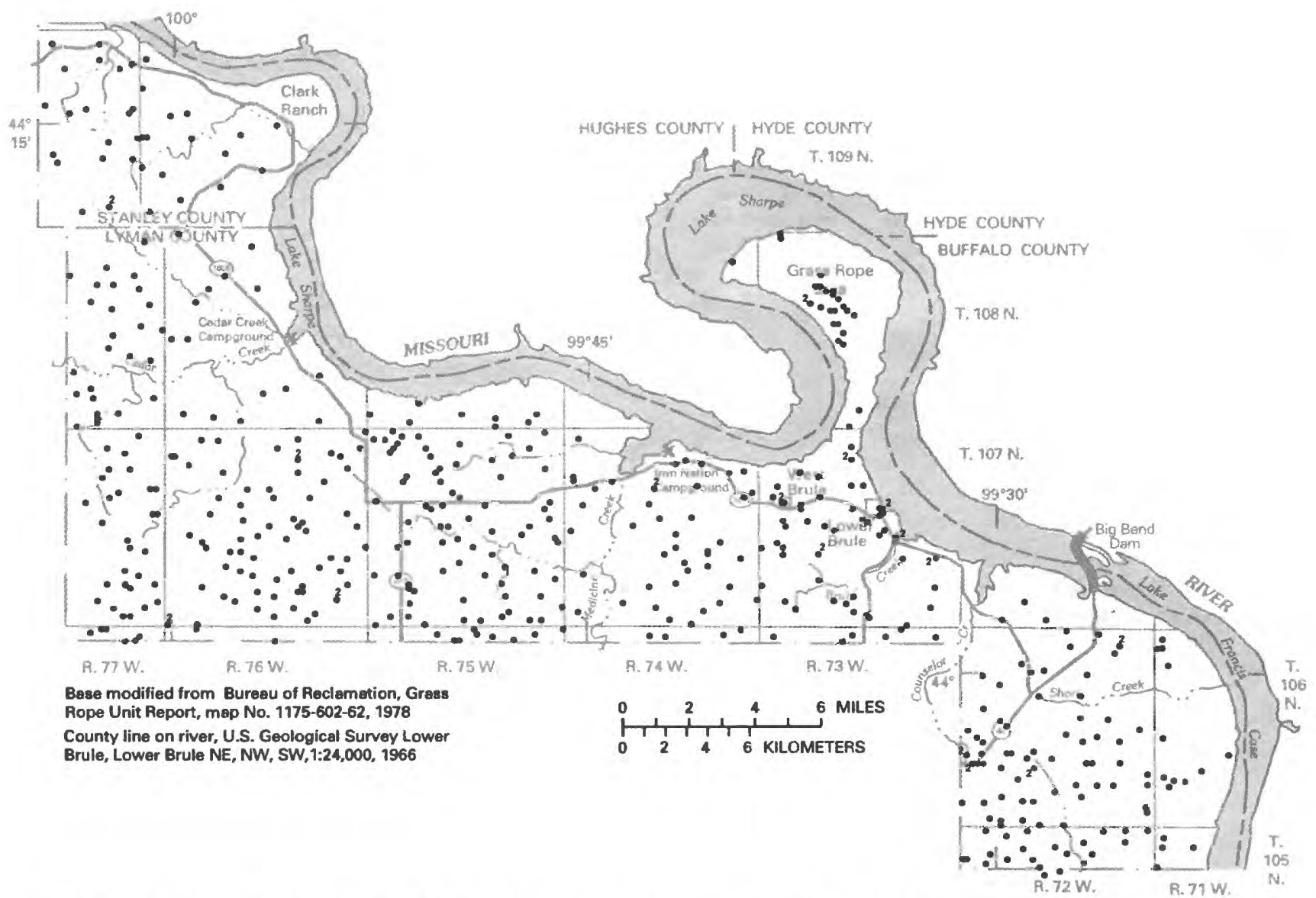

Figure 5. Locations of additional stock ponds and dugouts on the Lower Brule Indian Reservation from aerial photograph taken in August and September 1991.

Many of the stock ponds and dugouts visited in 1993 could not be sampled because they were dry, were overgrown with vegetation, or the water in them was completely frozen. Field analyses of the samples collected during that inventory are shown in table 10. These results may not be representative because, as stated previously, the quality of water in these impoundments probably varies greatly throughout the year, depending on dilution by runoff, concentration of salts by evaporation, and influshing of soluble minerals and organic matter.

\section{Ground-Water Availability and Water Quality}

Ground water on the Lower Brule Reservation can be obtained from surficial deposits at selected locations and from bedrock units that underlie the entire area. The quality of much of the ground water is less than desirable.

\section{Surficial Deposits}

The possibility of obtaining adequate supplies of good-quality water from surficial deposits is limited to parts of some terraces along the Missouri River (fig. 7). The sand and gravel deposits underlying the major terraces are good sources of water only where they are thick enough to extend below the water table (corresponding to the local water level in the Missouri River reservoirs). Thus, the ancient channels across the La Roche Terrace and Grass Rope area are potentially good sources of water. Locally, yields of 50 gallons per minute may be obtained from wells screened within the terrace deposits. The quality of the water varies, depending upon the source of the water and the permeability and local composition of 


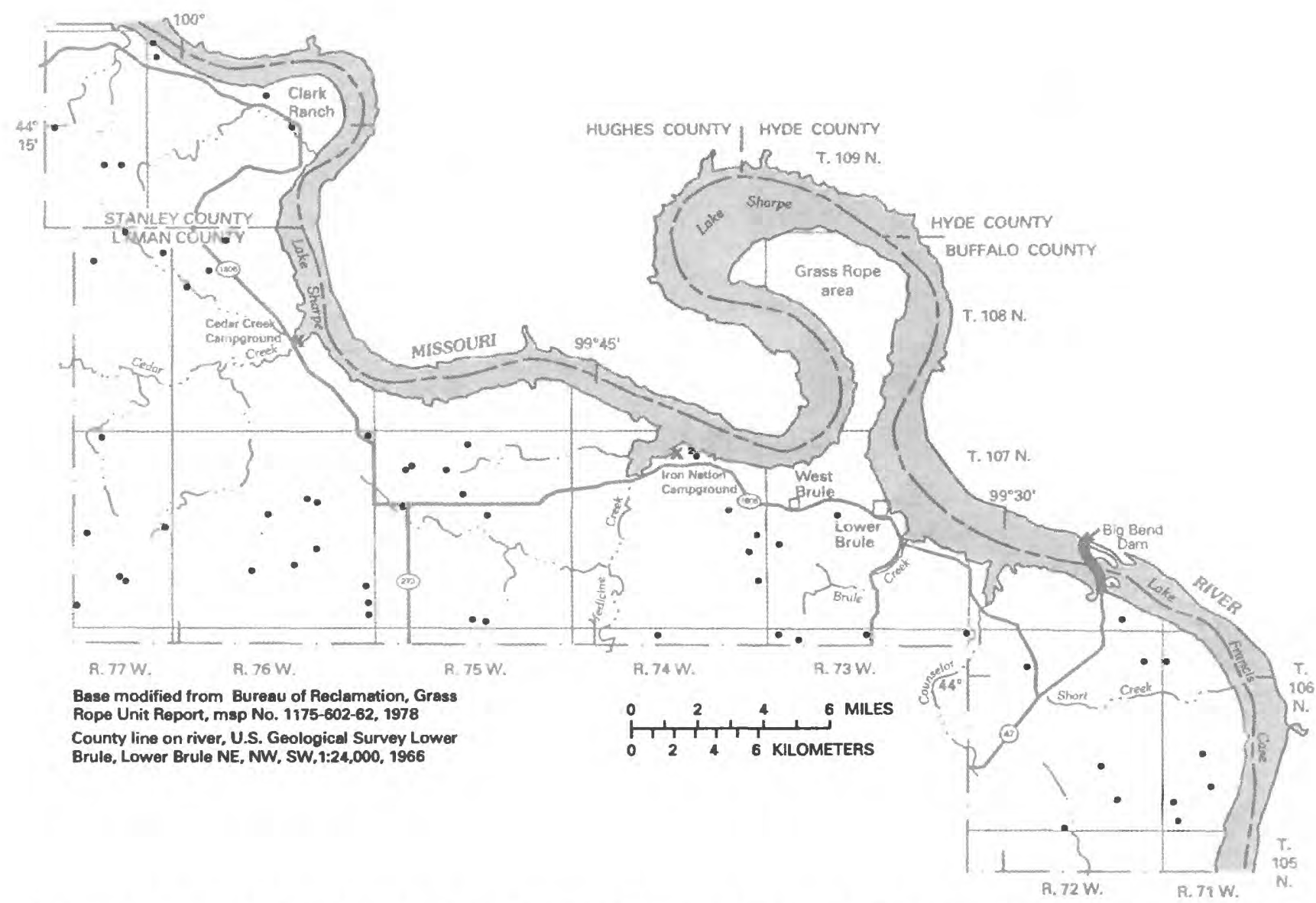

Figure 6. Locations of stock dams that were breached or possibly breached on the Lower Brule Indian Reservation as of 1993.

Table 10. Field analyses of water samples collected from stock ponds and dugouts on the Lower Brule Indian Reservation during February 1993

$[--$, not analyzed]

\begin{tabular}{|c|c|c|c|c|c|c|c|}
\hline Location & pH & $\begin{array}{c}\text { Alkalinity } \\
\text { (milligrams } \\
\text { per liter) }\end{array}$ & $\begin{array}{c}\text { Specific } \\
\text { conductance } \\
\text { (microsiemens } \\
\text { per centimeter } \\
\text { at } 25 \text { degrees } \\
\text { Celsius) }\end{array}$ & Location & $\mathrm{pH}$ & $\begin{array}{c}\text { Alkalinity } \\
\text { (milligrams } \\
\text { per liter) }\end{array}$ & $\begin{array}{c}\text { Specific } \\
\text { conductance } \\
\text { (microsiemens } \\
\text { per centimeter } \\
\text { at } 25 \text { degrees } \\
\text { Celsius) }\end{array}$ \\
\hline 106-72- 4BCBC & 7.06 & 51 & 700 & 107-76- $1 \mathrm{ABBC}$ & 7.02 & 154 & 700 \\
\hline $107-73-16 \mathrm{ACDC} / \mathrm{D}^{1}$ & 7.92 & 514 & 710 & 107-77-14DCBA & 9.13 & _. & 100 \\
\hline 107-73-19BCAC & 8.07 & -- & 200 & & & & \\
\hline 107-73-30CCAC & 7.82 & 51 & 600 & 107-77-23DBBA/B & 8.15 & -- & 600 \\
\hline 107-74-31BBAA & 7.04 & - & 1,320 & 109-76-29BBBB/C & 7.40 & 205 & 500 \\
\hline 107-75-3CADA/D & 7.43 & 223 & 300 & 109-76-33CBDD & 7.64 & 120 & 100 \\
\hline $\begin{array}{l}\text { 107-75-11DDCA } \\
\text { and DDDB }\end{array}$ & 7.22 & 240 & 270 & and CCAA & 1.04 & & \\
\hline
\end{tabular}

l" $\mu$ infers that dam is located on divide between quadrant ACDC and quadrant ACDD (see page vi for further explanation). 


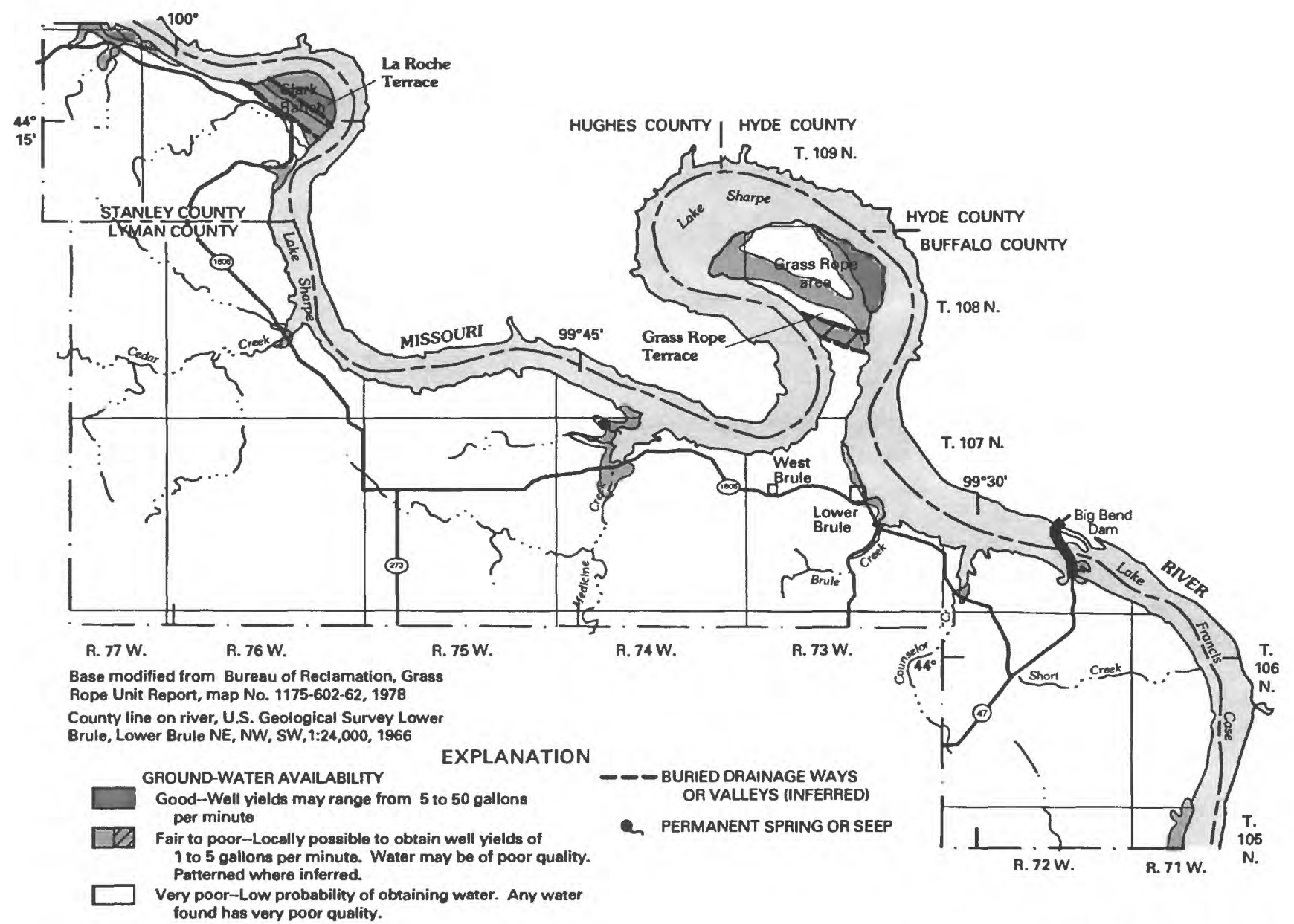

Figure 7. Ground-water availability from surficial deposits on the Lower Brule Indian Reservation (modified from Howells, 1974).

the deposits. The better quality water comes from Lake Sharpe through coarse, clean gravel; the poorer quality water probably infiltrates through clayey material and may occur on top of the Pierre Shale bedrock surface beneath the terraces.

Where the base of the terrace is above the reservoir water level, the high permeability of the sand and gravel and the steep slope of the underlying bedrock surface permit rapid discharge of infiltrating precipitation to the Missouri River. Thus, most of the areas within the Grass Rope and La Roche terraces, for example, do not contain large or dependable supplies of water although locally the sand and gravel may be more than 90 feet thick. If wells were completed in the deeper parts of these deposits near the bedrock surface, small to moderate supplies of water of varying quality might be obtained; but such wells might go dry in late summer or fall.
Pierre Shale, which is at or near the surface in much of the area, has almost no potential as a source of potable water. The Pierre Shale usually will not yield water to wells; but whẹre water can be obtained, generally it is of limited quantity and saline. The shale, which is relatively impermeable, acts as a barrier to the downward movement of ground water, causing the water to move laterally to points of discharge at contact springs. Many small springs and seeps occur along contacts between the Pierre Shale and overlying surficial deposits. The quality of water from these contact springs ranges from fairly good to very poor. Most springs and seeps are temporary and go dry by late summer. Colluvium and alluvium along streams draining areas where shale is at the surface commonly are composed of clay or silty clay and are relatively impermeable; the water in these deposits usually is of poor quality. 
Glacial till is not much better than the Pierre Shale as a potential source of water. Locally, thin beds or lenses of gravel, sand, or silt within the till may yield small quantities of water, typically of poor quality.

Alluvium along several streams, particularly at the mouths of Cedar, Medicine, Brule, and Counselor Creeks, potentially may yield small quantities of water. However, this water also commonly is of poor quality.

Very few permanent springs or seeps exist on the reservation. Most of the springs and seeps mapped by the Bureau of Indian Affairs (1964) soil and range surveys are temporary and go dry by late summer. Some are seepage from nearby stock ponds or dugouts. Howells (1974) states that all permanent springs on the Lower Brule Reservation have flows of less than 5 gallons per minute. The location of the one permanent spring on the Lower Brule Reservation that is listed in the USGS data base is shown in figure 7.

The quality of water in surficial deposits varies widely, often within short distances. For example, water from a well at one locality was of calcium bicarbonate type while water from a well a quarter mile away, apparently screened in the same water-bearing zone, was of sodium sulfate type. Where such water supplies have been in contact with the Pierre Shale, water quality may be poor, and dissolved-solids concentration can exceed $4,000 \mathrm{mg} / \mathrm{L}$. Such variations make it difficult to predict water quality reliably when selecting well sites. In general, the cleaner, coarser, and more permeable aquifer material tends to yield better quality water. The USGS water-quality data base contains only one analysis each for seven shallow wells located on the reservation. These analyses, from samples taken in 1969, are presented in table 11. Comparing the data in table 11 to the EPA drinking-water regulations in table 7 indicates that dissolved-solids concentration exceeded the Secondary Maximum Contaminant Level (SMCL) for all seven wells. The SMCL for sulfate was exceeded in six wells, the SMCL for iron and manganese was exceeded in two wells, the SMCL for chloride was exceeded in one well, and the Maximum Contaminant Level (MCL) for nitrate (as N) was exceeded in one well.

\section{Bedrock Units}

Artesian bedrock aquifers underlie the entire study area. These aquifers are part of a thick sequence of sedimentary units that extend across the State.

The most important aquifers are contained in the Dakota Sandstone, the Inyan Kara Group, the Minnelusa Formation, and the Madison Group. Hydraulic head commonly increases with stratigraphic depth in these major aquifers, which are shown in figures $8,9,10$, and 11 . Thus, at a given site, greater artesian pressure generally can be obtained by tapping a deeper aquifer (where present) rather than a shallower aquifer.

The Dakota Sandstone is the most intensively developed aquifer in the State. On the Lower Brule Reservation, about 40 percent of wells in use tap this aquifer. Hydraulic head in the Dakota Sandstone has been declining (fig. 12) during the past 100 years and will continue to do so as long as aquifer withdrawals exceed recharge. Intensive development of deeper aquifers and the consequent decline in artesian pressure in them will cause an additional drop in head in the Dakota because most recharge to the Dakota currently is from the deeper aquifers. The average rate of decline in the Dakota currently is less than 1 foot per year (Winter, 1994), which may indicate that artesian pressure is stabilizing.

Most well owners are aware of the value of conserving and maintaining artesian pressure of the aquifers and many have equipped their flowing wells with control valves. Even though flow is reduced below the maximum rate, many wells flow at larger rates than are actually needed. Most wells drilled within the last 30 years are cased with copper or plastic because black iron or steel casings tend to deteriorate rapidly and corrode in permeable beds containing more saline water. Thus, copper or plastic casings tend to reduce long-term well costs by lengthening useful well life, as well as conserving pressure and maintaining original water quality.

Wells completed in the Dakota Sandstone yield moderately saline (more than $3,000 \mathrm{mg} / \mathrm{L}$ dissolved solids) sodium chloride type water that commonly is gassy. Some stock wells yield enough gas to support a flame. 
Table 11.--Physical and chemical data for water samples collected at seven shallow wells on the Lower Brule Indian Reservation, 1969

[Reported in milligrams per liter (mg/L) except as indicated. ${ }^{\circ} \mathrm{C}$, degree Celsius; $\mu \mathrm{S} / \mathrm{cm}$, microsiemens per centimeter at $25^{\circ} \mathrm{C} ; \mu \mathrm{g} / \mathrm{L}$, micrograms per liter; $\mathrm{ND}$, analyzed for but not detected; --, no data; <, less than]

\begin{tabular}{|c|c|c|c|c|c|c|c|}
\hline Parameter & $\begin{array}{l}\text { 109N76W } \\
13 \mathrm{BBBB}^{1}\end{array}$ & $\begin{array}{l}109 N_{77 W} \\
2 A D D^{1}\end{array}$ & $\begin{array}{c}107-73- \\
22 A D C C^{2}, 3.4\end{array}$ & $\begin{array}{l}107-74- \\
3 \text { CBCB }^{5,6}\end{array}$ & $\begin{array}{c}107-74- \\
5 A B B C 1^{1,7}\end{array}$ & $\begin{array}{c}\text { 108-73- } \\
\text { 10BBBB1 }\end{array}$ & $\begin{array}{c}108-76- \\
22 \text { ACDB }^{5,8}\end{array}$ \\
\hline Sample date & $06-18-69$ & $06-19-69$ & $08-05-69$ & $09-24-69$ & $06-18-69$ & $06-18-69$ & $09-18-69$ \\
\hline Depth of well (feet) & 80 & 35 & 45 & 22 & 6 & 47 & 28 \\
\hline $\begin{array}{l}\text { Elevation of land-surface datum } \\
\text { (feet above sea level) }\end{array}$ & 1,456 & 1,475 & 1,445 & 1,425 & 1,460 & 1,483 & 1,428 \\
\hline Specific conductance $(\mu \mathrm{S} / \mathrm{cm})$ & 3,490 & 4,880 & 3,220 & -- & 821 & 3,600 & -- \\
\hline $\mathrm{pH}$ (standard units) & 7.6 & 7.7 & 7.9 & 7.9 & 8.0 & 8.0 & 7.5 \\
\hline Temperature $\left({ }^{\circ} \mathrm{C}\right)$ & 12.5 & 11.1 & -- & -- & 13.0 & 10.6 & -- \\
\hline Carbon dioxide, dissolved & 12 & 12 & -- & -- & -- & - & -- \\
\hline Hardness, total (as $\mathrm{CaCO}_{3}$ ) & 2,100 & 1,050 & 967 & 2,670 & 261 & 963 & 874 \\
\hline 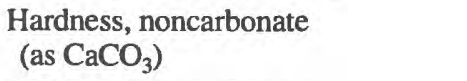 & 1,860 & 741 & 671 & -- & 0.0 & 592 & - \\
\hline Alkalinity (as $\mathrm{CaCO}_{3}$ ) & 242 & 311 & 296 & -- & 326 & 222 & - \\
\hline Solids, dissolved, residue at $180^{\circ} \mathrm{C}$ & 3,560 & 3,770 & 2,780 & 4,500 & 521 & 2,920 & 2,270 \\
\hline $\begin{array}{l}\text { Solids, dissolved, sum of } \\
\text { constituents }\end{array}$ & 3,170 & 3,510 & 2,560 & -- & 518 & 2,630 & - \\
\hline Calcium, dissolved & 510 & 280 & 228 & 337 & 38 & 155 & 83 \\
\hline Magnesium, dissolved & 202 & 86 & 92 & 445 & 41 & 140 & 162 \\
\hline Sodium, dissolved & 170 & 750 & 469 & - & 85 & 500 & -- \\
\hline Sodium, percent & 15 & 60 & 52 & -- & 41 & 53 & -- \\
\hline Sodium-adsorption ratio & 1.61 & 10.1 & 6.46 & -- & 2.29 & 7.01 & -- \\
\hline Potassium, dissolved & 12 & 15 & 6.3 & -- & 3.6 & 11 & -- \\
\hline Bicarbonate & 295 & 379 & 361 & -. & 398 & 452 & -- \\
\hline Carbonate & 0.0 & 0.0 & 0.0 & 0.0 & 0.0 & 0.0 & 0.0 \\
\hline Sulfate, dissolved & 2,010 & 1,540 & 1,510 & 2,600 & 112 & 1,300 & 1,130 \\
\hline Chloride, dissolved & 86 & 620 & 64 & 65 & 13 & 240 & 107 \\
\hline Fluoride, dissolved & 0.8 & 1.2 & .55 & -- & 1.0 & 1.2 & - \\
\hline Silica, dissolved & 19.4 & 26 & -- & -- & 25.8 & 27.7 & -- \\
\hline Nitrogen, nitrate, dissolved (as N) & 0.11 & 0.70 & ND & 27.1 & 0.68 & 7.1 & 0.25 \\
\hline $\begin{array}{l}\text { Nitrogen, nitrate, dissolved } \\
\left(\text { as } \mathrm{NO}_{3}\right)\end{array}$ & 0.5 & 3.1 & ND & 120 & 3.0 & 31.6 & 1.1 \\
\hline Arsenic $(\mu \mathrm{g} / \mathrm{L})$ & -- & -- & ${ }^{9}<10$ & -- & - & -- & -- \\
\hline Boron, dissolved $(\mu \mathrm{g} / \mathrm{L})$ & 260 & 1,830 & -- & -- & 180 & 840 & - \\
\hline Iron, dissolved $(\mu \mathrm{g} / \mathrm{L})$ & 5,460 & 30 & 8,400 & $<5$ & 40 & 190 & $<5$ \\
\hline Manganese, dissolved $(\mu \mathrm{g} / \mathrm{L})$ & 6,800 & 20 & 550 & $<5$ & $<5$ & $<5$ & $<5$ \\
\hline Selenium, dissolved $(\mu \mathrm{g} / \mathrm{L})$ & $<5$ & $<5$ & ${ }^{9} 4.6$ & -- & $<5$ & 20 & - \\
\hline $\begin{array}{l}\text { Sodium carbonate, residual } \\
\text { (as } \mathrm{CaCO}_{3} \text { ) }\end{array}$ & 0.00 & 0.00 & 0.00 & -- & 1.21 & 0.00 & - \\
\hline
\end{tabular}

${ }^{1}$ Analysis by U.S. Geological Survey laboratory.

${ }^{2}$ Analysis by South Dakota Department of Health, Pierre, except as indicated.

${ }^{3}$ Well can go dry for extended periods. Known to have heen dry in late summer and fall of 1967.

${ }^{4}$ Well in Lower Brule town park.

${ }^{5}$ Analysis by U.S. Corps of Engineers laboratory, Omaha, Nebr.

${ }^{6}$ Iron Nation campground well \#1.

${ }^{7}$ Developed spring.

${ }^{8}$ Cedar Creek campground.

${ }^{9}$ Analysis by South Dakota Water Resources Institute Laboratory, Brookings. 

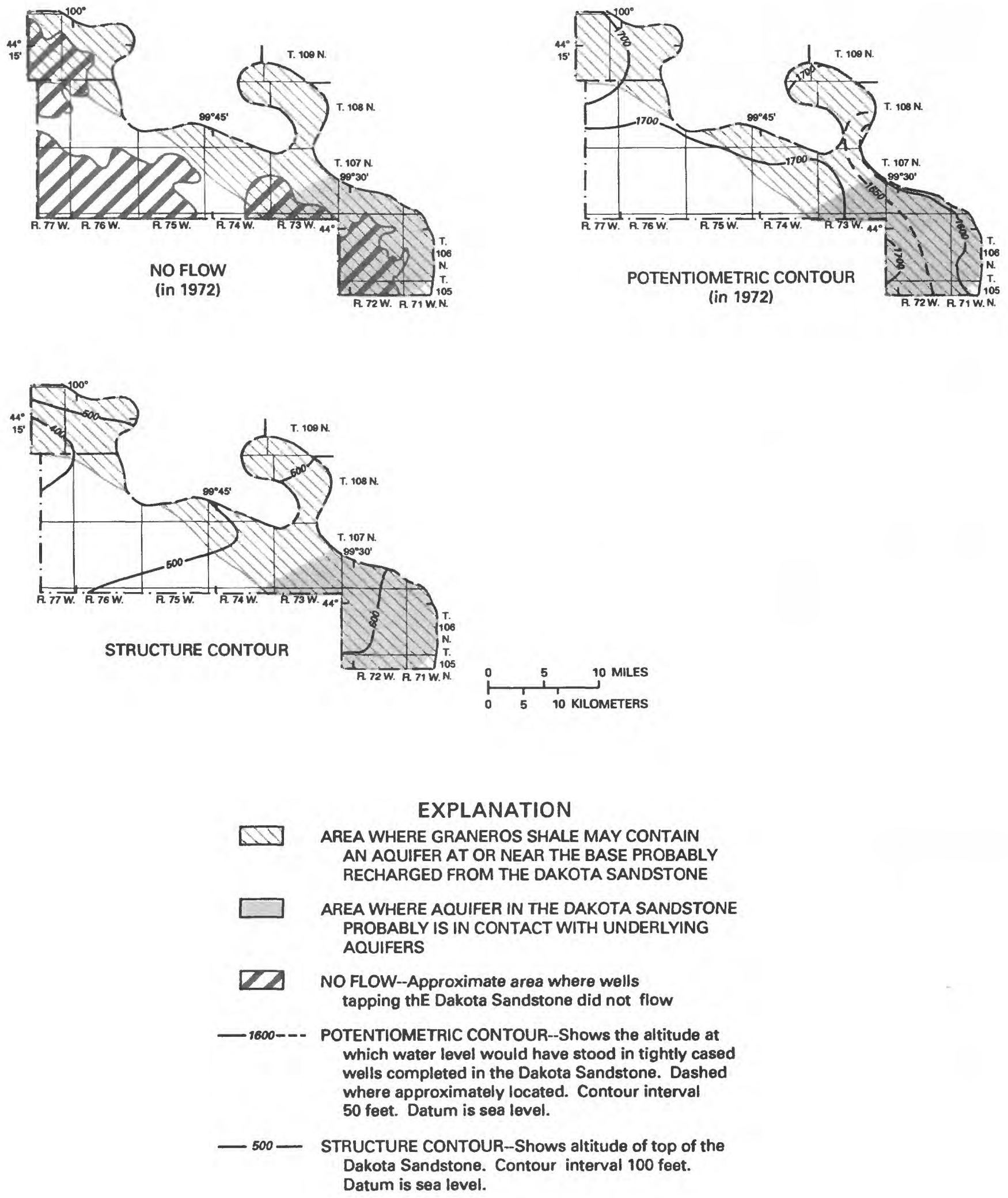

Figure 8. Hydrogeology of the Dakota Sandstone on the Lower Brule Indian Reservation (modified from Howells, 1974). 

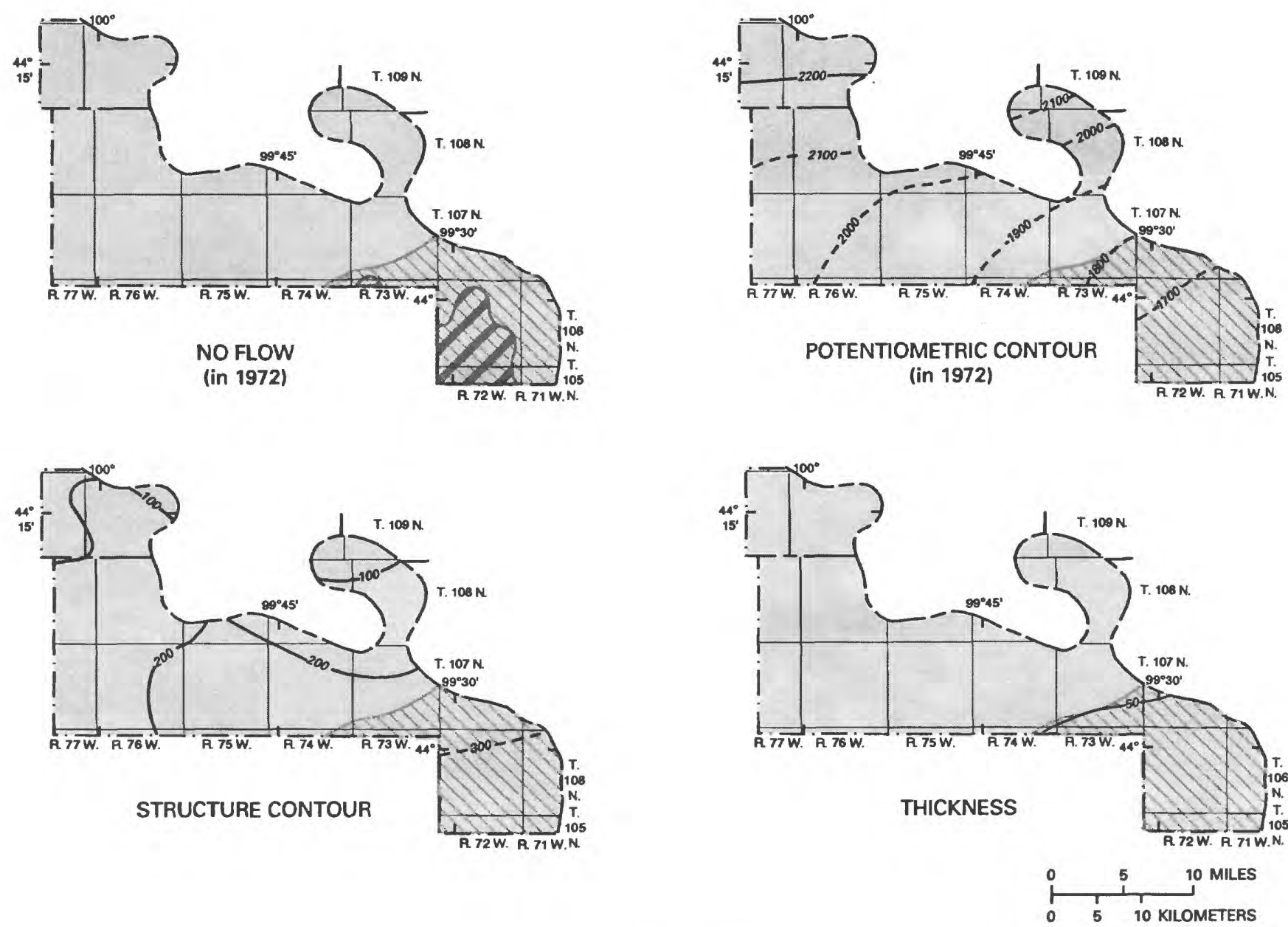

\section{EXPLANATION}

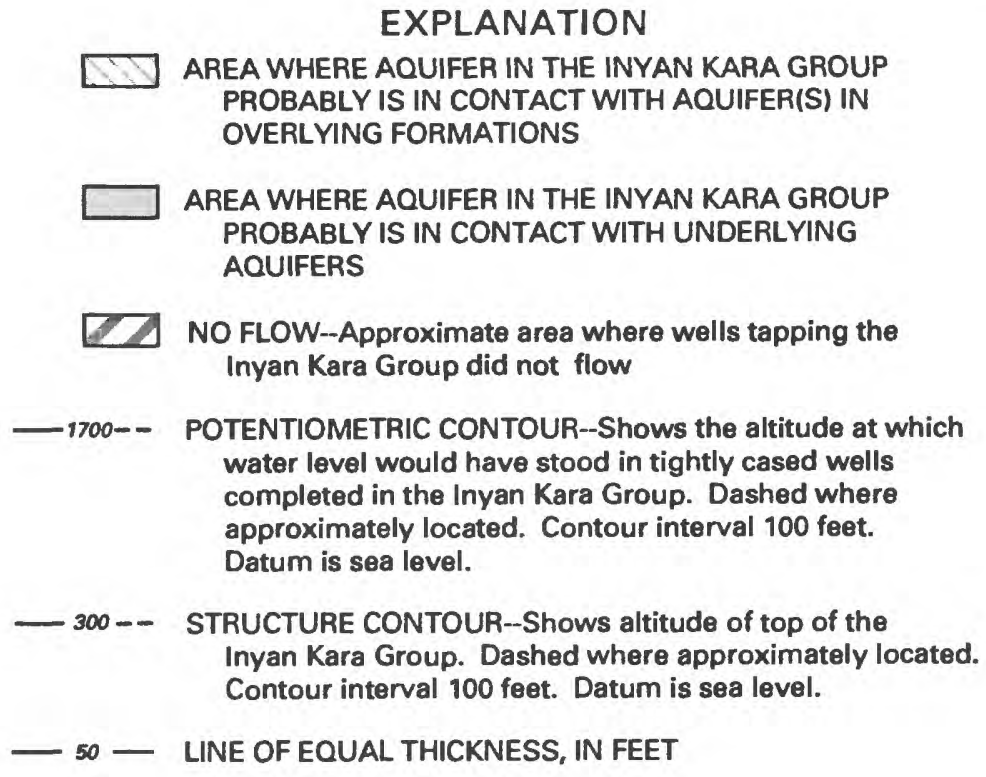

Figure 9. Hydrogeology of the Inyan Kara Group on the Lower Brule Indian Reservation (modified from Howells, 1974). 

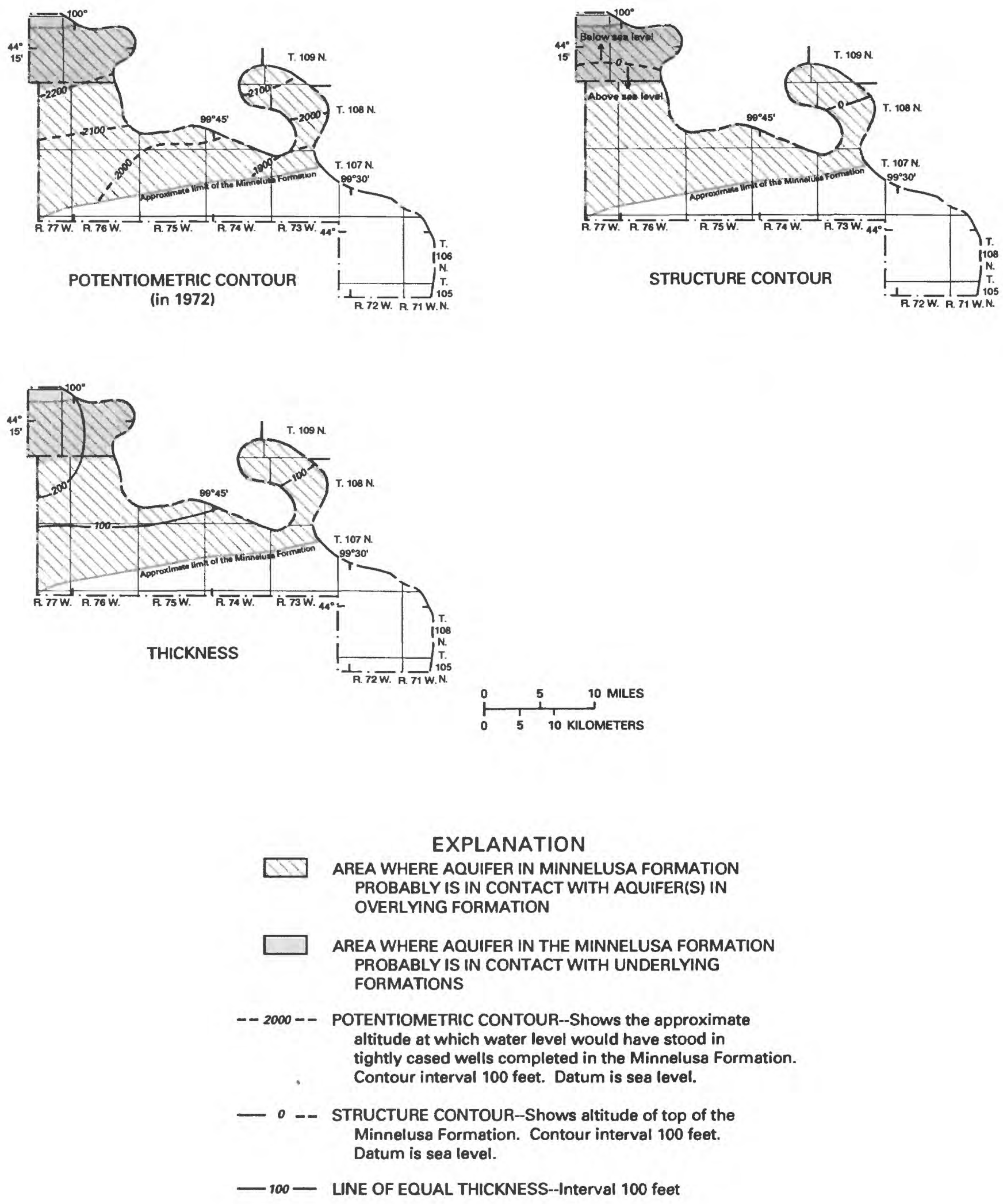

Figure 10. Hydrogeology of the Minnelusa Formation on the Lower Brule Indian Reservation (modified from Howells, 1974). 

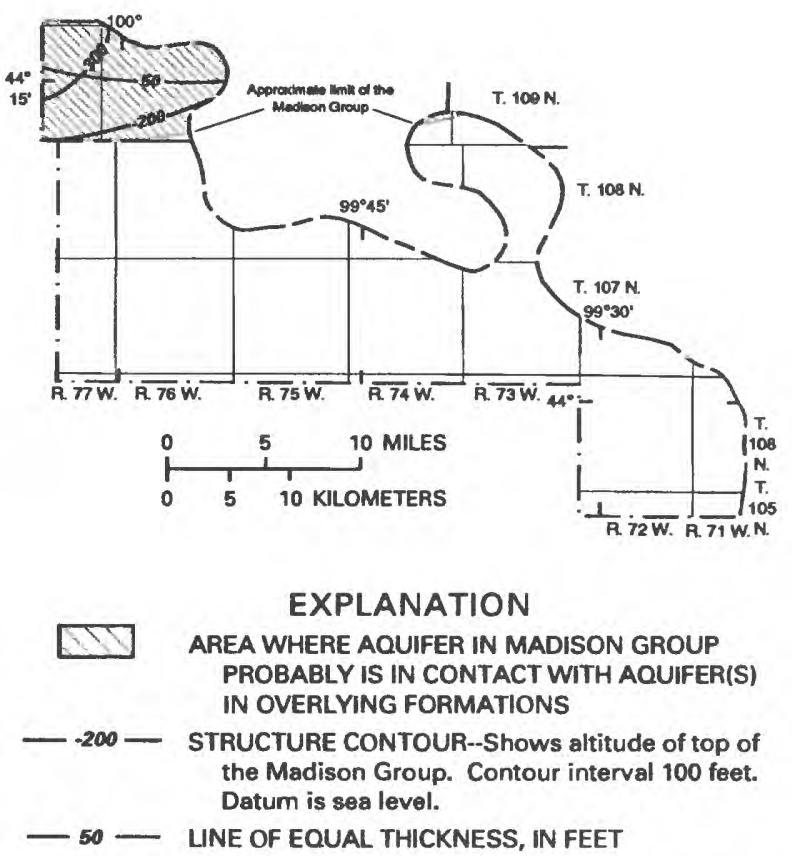

Figure 11. Hydrogeology of the Madison Group on the Lower Brule Indian Reservation (modified from Howells, 1974).

The geochemistry of water in the Dakota Sandstone appears to be complex. Within or near the reservation, wells tapping the Dakota may yield sodium chloride, sodium bicarbonate, or calcium magnesium sulfate type water. Between the regions where each of these types of water predominates are areas of mixed water types such as sodium sulfate or sodium chloride bicarbonate and others. The chemical data also indicate that the Dakota is receiving major recharge where it is in hydraulic contact with underlying aquifers. Calcium magnesium sulfate type water occupies a larger proportion of the basal one-third of the formation than in the top one-third, a natural consequence of its movement into the Dakota from below (fig. 13). Also, the pattern of major-ion distribution generally parallels the area where the aquifer in the Dakota is in contact with underlying aquifers. The source of predominantly sodium bicarbonate type water, present in the Dakota a few miles west of the Lower Brule Reservation, is unknown (L.W. Howells, USGS, oral commun., 1994). Sodium chloride type water probably is connate (trapped in the Dakota when the formation was deposited).
The proportion of the Dakota Sandstone that yields calcium magnesium sulfate type water probably is increasing. As sodium chloride or sodium bicarbonate type water is withdrawn by wells, recharge is increased from underlying aquifers (L.W. Howells, USGS, oral commun., 1994). Some wells in central South Dakota have shown large changes in water quality over the years. Commonly, these changes have been increases in the proportion of calcium, magnesium, and sulfate.

Aquifers deeper than the Dakota beneath the reservation generally contain extremely hard, moderately

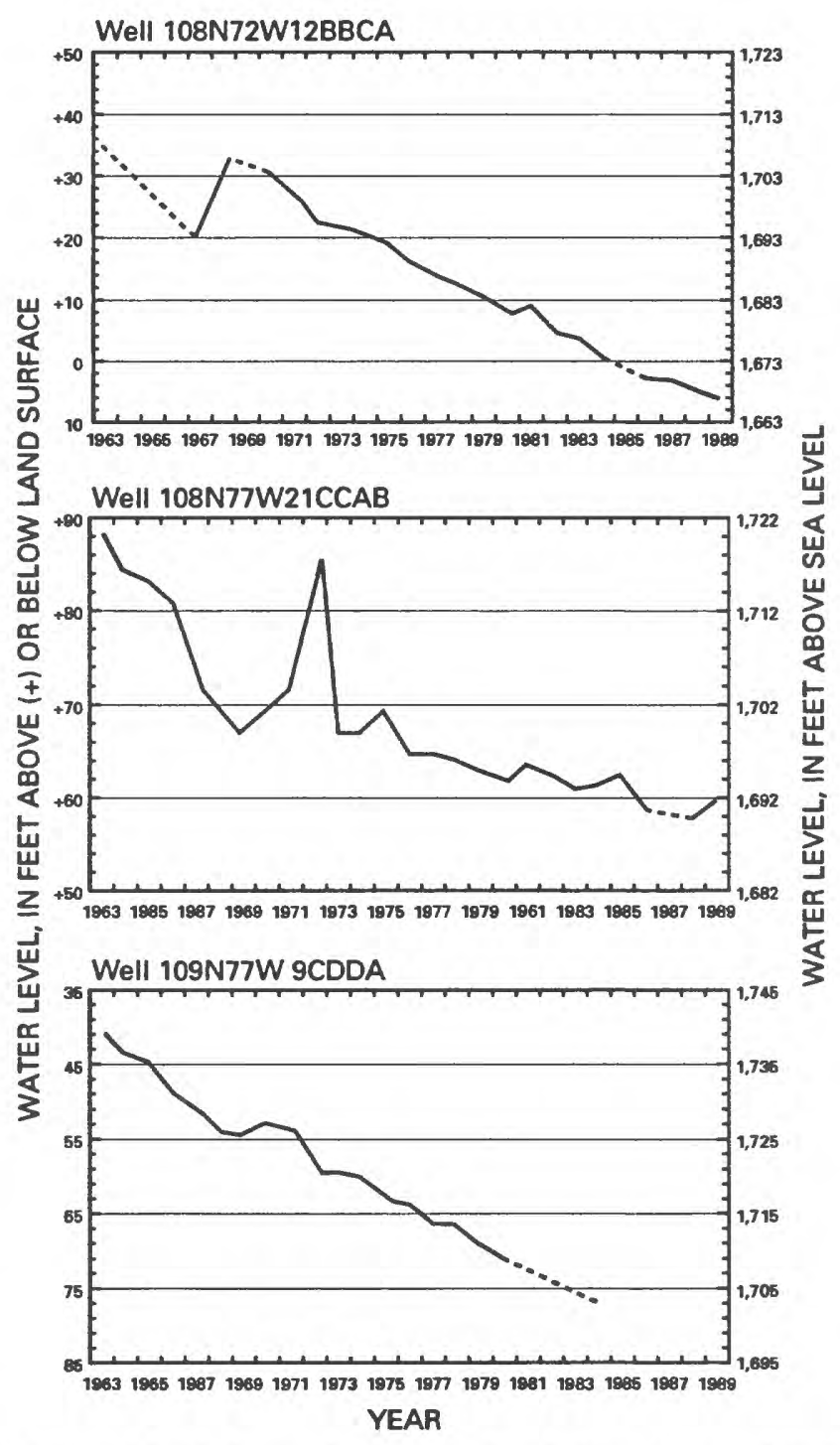

Figure 12. Hydrographs of water levels in wells completed in the Dakota Sandstone (from Winter, 1994; dashed where time between water-level measurements is greater than 1 year). 

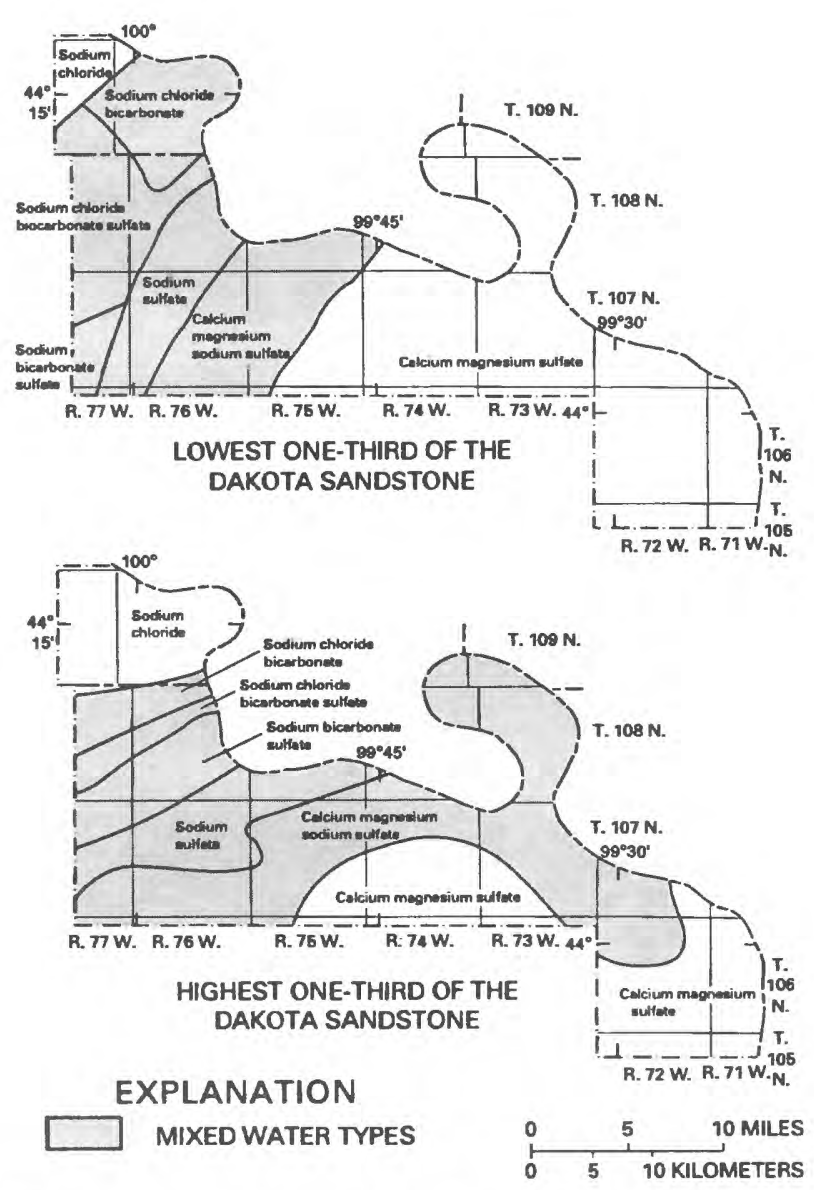

Figure 13. Water types in the Dakota Sandstone on the Lower Brule Indian Reservation as of 1972 (modified from Howells, 1974).

saline, calcium magnesium sulfate type water. Specific conductance ranges from about 2,200 to $2,600 \mu \mathrm{S} / \mathrm{cm}$, hardness ranges from about 1,200 to more than $1,400 \mathrm{mg} / \mathrm{L}$, sulfate concentration ranges from 1,200 to $1,500 \mathrm{mg} / \mathrm{L}$, fluoride concentration is more than $2.8 \mathrm{mg} / \mathrm{L}$, nitrate (as $\mathrm{N}$ ) concentration is less than $1 \mathrm{mg} / \mathrm{L}$, and boron concentration usually is less than $250 \mu \mathrm{g} / \mathrm{L}$ (U.S. Geological Survey, unpub. data).

\section{Well Inventory}

During the summer and fall of 1992, a well inventory was made on the Lower Brule Reservation. The purpose of the inventory was to document the location, condition, and use of water from wells located within the boundaries of the reservation. Of the 287 wells on the reservation listed in the USGS data base, 95 percent were visited by USGS personnel.
Well owners were asked for the age and depth of the well, the use of the water, the drilling company, a copy of the driller's log if available, permission to sample the water, and permission to measure the depth to water if the well was not flowing. Wells that had been destroyed, were unusable, or abandoned were noted. Forty-five of the 287 wells had been, or were reported to have been, destroyed or abandoned and another 103 were test holes that had never been completed as wells. Therefore, the USGS groundwater data base lists 139 wells in use, or in usable condition, on the reservation. Of these, 101 were in use in 1992.

The wells and test holes are numbered according to a system based on the Federal land survey for eastern South Dakota (see p. vi). The locations of the 139 usable wells on the Lower Brule Reservation in 1992 are shown in figure 14. The owner(s), locations, and other information for those wells are presented in table 12.

Ground-water-quality samples were taken where feasible. Field water-quality analyses for those wells are shown in table 13. Comparing the data in table 13 to the EPA drinking-water regulations in table 7 shows that one well (located in 108-76-8AABC) had a chloride concentration that exceeded the SMCL of $250 \mathrm{mg} / \mathrm{L}$, and one well (located in 106-72-26BCCA) had a $\mathrm{pH}$ value outside the SMCL range of 6.5 to 8.5 .

\section{INDIAN WATER RIGHTS}

Indian water rights have evolved from the Winters Doctrine (Winters v. United States, 207 U.S. 564, 1908) (Foster, 1978). In a historic Supreme court decision, it was held that, when Indian reservations were established, the Federal Government also reserved the water necessary to make the land productive. States could not appropriate such water to other users. The court held that the amount of water reserved for Indian use was that amount sufficient to irrigate all the practically arable reservation land. Although Winters v. United States was specifically applied to surface water, the Supreme Court also addressed the issue with regard to ground water (Cappaert v. United States, 426 U.S. 128, 1976). Water-resources management in the United States can be complicated, especially in the West, where water shortages are common. 


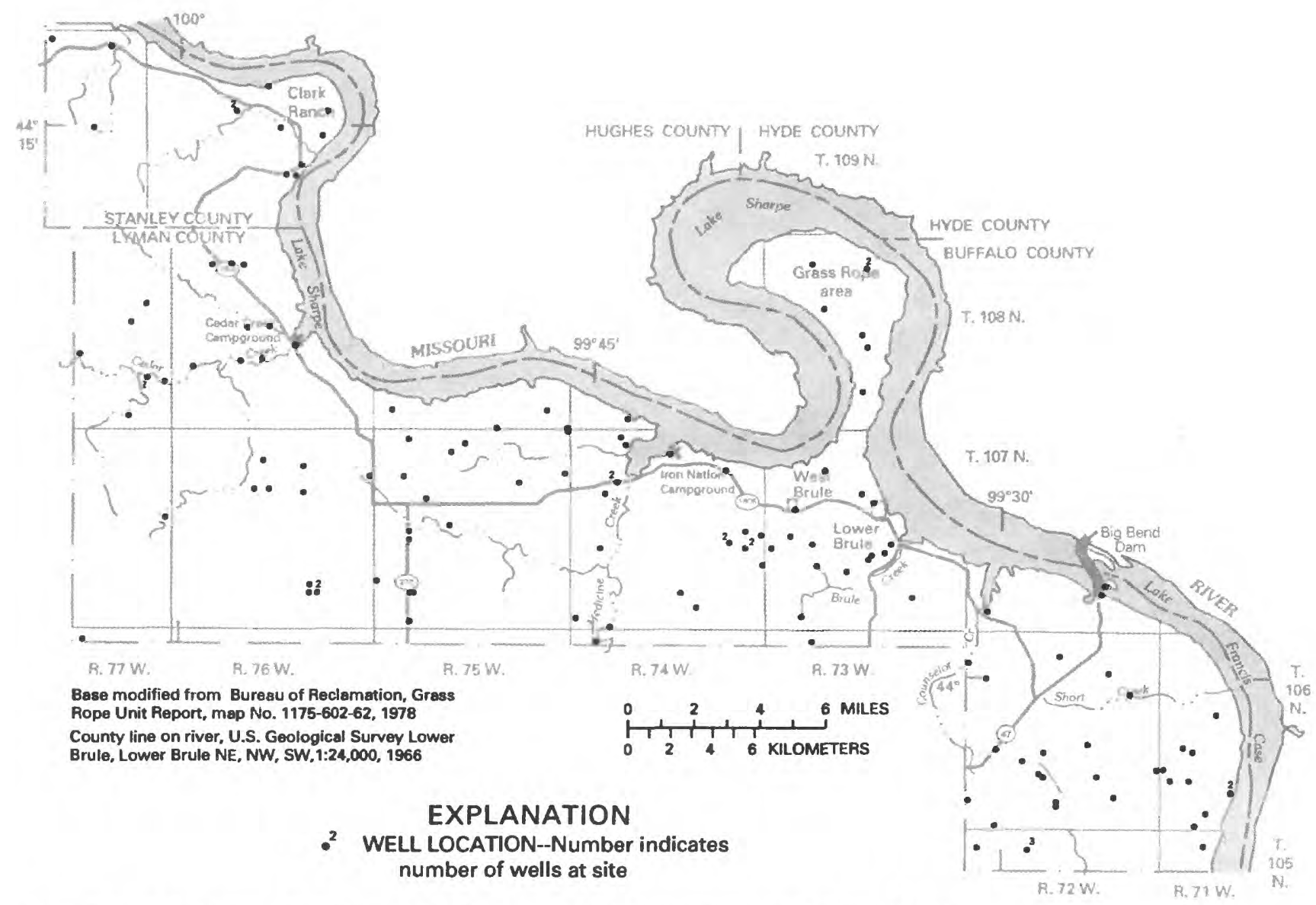

Figure 14. Locations of usable wells on the Lower Brule Indian Reservation as of 1992.

In January 1993, the Mni Sose Intertribal Water Rights Coalition was formed. The coalition is a board whose members are representatives of 22 of the 28 tribes in the Missouri River Basin, including the Lower Brule Sioux Tribe. The primary purpose of the Mni Sose Coalition is to educate its membership about the nature, character, and extent of water issues pertinent to American Indians. The Winters Doctrine reserved Indian rights to the use of water from the Missouri River and tributary streams, and ground water that underlies, borders, or traverses reservations. The coalition will assist member tribes in developing plans to protect, preserve, utilize, administer, manage, and develop their water resources for the economic and social benefit of all tribal members. Currently, the coalition is working to develop a policy statement that defines the Federal Government's role in Indian water issues.
South Dakota Codified Law (SDCL) 46-1-3 declares that all water within the State is the property of the people of the State, but the right to the use of water may be acquired by appropriation as provided by law. SDCL 46-2-11 vests the authority to regulate and control the development, conservation, and allotment of waters of the State with the Water Management Board. However, the question as to who (the State of South Dakota, Federal Government, or Indian Tribes) has the authority to issue and enforce water rights on Tribal and individual allotted trust lands in South Dakota remains unanswered. A water right, as defined by South Dakota Water Law, is not legal title to the water, but the legal right to use it in a manner specified by State law. Permits are issued according to the procedures established by the delegated permit authority. The Lower Brule Sioux Tribe does not necessarily concur with the State's 
Table 12. Usable wells on the Lower Brule Indian Reservation, 1992

[Not included are any wells found, or reported, to be unable to produce water. --, no data; >, greater than]

\begin{tabular}{|c|c|c|c|c|c|}
\hline Owner & Well number & $\begin{array}{l}\text { Latitude/ } \\
\text { longitude }\end{array}$ & $\begin{array}{c}\text { Primary use } \\
\text { of water } \\
\text { reported in } 1992\end{array}$ & $\begin{array}{l}\text { Depth } \\
\text { of well } \\
\text { (feet) }\end{array}$ & $\begin{array}{l}\text { Geologic unit(s) } \\
\text { in which well } \\
\text { is completed }\end{array}$ \\
\hline Bart Blum & 105-71- 5CABB & 4355240992252 & Domestic & 725 & Graneros and Dakota \\
\hline John Christenson & 105-72- 5DADA & 4355330993000 & Domestic & 23 & Pierre \\
\hline John Christenson & 105-72- 5DADB & 4355300992915 & Unused & -- & -- \\
\hline John Christenson & 105-72- 5DADD & 4355320992910 & Unused & 1,360 & Dakota \\
\hline Frank Woster & $105-72-6 \mathrm{CA}$ & 4355360993111 & Domestic & 1,280 & $\begin{array}{l}\text { Dakota (and } \\
\text { Graneros }{ }^{2} \text { ) }\end{array}$ \\
\hline Lawrence Thompson & 106-71-17DBAA & 4359030992217 & Domestic & 850 & $\begin{array}{l}\text { Graneros (and } \\
\text { Dakota }^{2} \text { ) }\end{array}$ \\
\hline L. Thompson & 106-71-19DABD & 4358080992320 & Unused & 916 & Dakota \\
\hline C. Baker & 106-71-20СВCВ & 4358070992307 & Domestic & -- & -- \\
\hline Brule Cattle Company & $106-71-28 \mathrm{CCDB}$ & 4356590992144 & Stock & 829 & Dakota \\
\hline City of Lower Brule & $106-71-28 C D$ & 4357020992132 & Unused & 760 & $\begin{array}{c}\text { Dakota (and } \\
\text { Graneros }{ }^{2} \text { ) }\end{array}$ \\
\hline Brule Cattle Company & 106-71-30BBCD & 4357350992405 & Unused & 973 & Dakota \\
\hline Theodore Bolman & 106-71-30СABA & 4357190992356 & Domestic & 946 & Dakota \\
\hline Keith Johnson & 106-71-30DAAB & 4357220992312 & Domestic & -- & Dakota $^{2}$ \\
\hline Lawrence Thompson & 106-71-32CAA & 4356240992236 & Domestic & 715 & $\begin{array}{l}\text { Graneros (and } \\
\text { Dakota }^{2} \text { ) }\end{array}$ \\
\hline Richard Menzie & 106-71-32CCCD & 4356070992300 & Domestic & -- & Dakota $^{2}$ \\
\hline R. Jandreau & 106-72- 4DDAD & 4400320992759 & Domestic & 1,310 & Dakota \\
\hline M. Karlen, Jr. & $106-72-6 \mathrm{CCCA}$ & 4400280993127 & Stock & 1,000 & Dakota \\
\hline Brule Cattle Company & 106-72- 7ACDB & 4400030993047 & Stock & 1,068 & Dakota \\
\hline C. Thompson & 106-72-11BDAA & 4400080992613 & Unused & 1,015 & Dakota \\
\hline C. Thompson & $106-72-12 \mathrm{CC}$ & 4359370992523 & Stock & 890 & Dakota \\
\hline Frank and Selma Bunker & 106-72-19DAAD & 4358130993029 & Unused & 1,275 & Dakota \\
\hline Frank and Selma Bunker & 106-72-20DDCB2 & 4357540992925 & Unused & 1,400 & Dakota \\
\hline Frank and Selma Bunker & 106-72-21CADC & 4358150992845 & Domestic & 1,270 & Dakota \\
\hline Tom Wilmot & 106-72-22ADCD & 4358160992657 & Stock & ${ }^{3} 1,000$ & -- \\
\hline Theodore Bolman & 106-72-25AAD & 4357380992425 & Stock & -- & Dakota $^{2}$ \\
\hline Tom Wilmot & $106-72-26 \mathrm{BCCA}$ & 4357260992640 & Stock & 1,360 & Dakota \\
\hline Lower Brule Cattle Association & 106-72-28BDBD & 4357260992839 & -- & 973 & Graneros \\
\hline John Bunker & 106-72-28BDDD2 & 4357250992807 & Domestic & 1,440 & Dakota \\
\hline Larry Larson & 106-72-31BBBB & 4356590993136 & Domestic & 1,300 & Dakota \\
\hline Clarence Thompson, Jr. & 106-72-31DDDC & 4356050992313 & Domestic & -- & Dakota $^{2}$ \\
\hline John Bunker & 106-72-33AACA & 4356470992808 & Unused & 1,350 & Dakota \\
\hline John Bunker & 106-72-33ADBC & 4356470992806 & Domestic & 1,275 & Dakota \\
\hline G. Williams & $106-72-35 \mathrm{ABB}$ & 4356520992604 & Unused & ${ }^{3} 1,000$ & $\begin{array}{l}\text { Dakota and/or } \\
\text { Graneros }\end{array}$ \\
\hline L.C. Knutsen & 106-73-5BCDA & 4400550993716 & Domestic & 19 & Pierre \\
\hline J. Ireland & 106-74- 6ACAD & 4400580994503 & Stock & 1,100 & Dakota \\
\hline C.F. Taylor & 106-77-3BBDD & 4401051000339 & Stock & 1,473 & Dakota \\
\hline U.S. Army Corps of Engineers & 107-72-26CADB & 4402260992618 & Public supply & 46 & Alluvium \\
\hline
\end{tabular}


Table 12. Usable wells on the Lower Brule Indian Reservation, 1992-Continued

\begin{tabular}{|c|c|c|c|c|c|}
\hline Owner & $\begin{array}{l}\text { Well number } \\
\text { (see page 10) }\end{array}$ & $\begin{array}{l}\text { Latitude/ } \\
\text { longitude }\end{array}$ & $\begin{array}{l}\text { Primary use } \\
\text { of water } \\
\text { reported in } 1992\end{array}$ & $\begin{array}{l}\text { Depth } \\
\text { of well } \\
\text { (feet) }\end{array}$ & $\begin{array}{l}\text { Geologic unit(s) } \\
\text { in which well } \\
\text { is completed }\end{array}$ \\
\hline U.S. Army Corps of Engineers & $107-72-26 \mathrm{CDCB}$ & 4402130992627 & Public supply & -- & Alluvium \\
\hline U.S. Army Corps of Engineers & $107-72-31 \mathrm{ACD}$ & 4401460993045 & Public supply & 36 & Alluvium \\
\hline Merrill Karlen & 107-73-8AACA & 4405330993637 & Stock & 1,220 & Dakota \\
\hline Ken Harmon & 107-73-9DDDB & 4404490993514 & Unused & -- & $\begin{array}{l}\text { Dakota and/or } \\
\text { Graneros }\end{array}$ \\
\hline Lower Brule Agency & 107-73-15BBD & 4404390993500 & -. & 894 & Dakota and Graneros \\
\hline Lower Brule (community well) & 107-73-18AD & 4404260993746 & Public supply & 1,410 & Dakota \\
\hline $\begin{array}{l}\text { Lower Brule Sioux Tribe Boy's } \\
\text { Ranch }\end{array}$ & 107-73-19AACC & 4403410993745 & -- & 1,474 & $\begin{array}{l}\text { Inyan Kara and } \\
\text { Dakota }\end{array}$ \\
\hline Hugh Wynia & 107-73-19CBAA2 & 4403250993830 & Domestic & 1,300 & Dakota \\
\hline Fred Reuer & 107-73-20BDDB & 4403380993702 & Stock & 1,236 & Dakota \\
\hline Lower Brule (city park) & 107-73-22ADCC2 & 4403280993410 & Public supply & 45 & Alluvium \\
\hline Boyd Gourneau & 107-73-22CCAC & 4403070993455 & Domestic & -- & -- \\
\hline Lower Brule Tribe & $107-73-22 \mathrm{CCCC}$ & 4403020993504 & Stock & -- & Dakota $^{2}$ \\
\hline Orville Langdeaux & 107-73-22DBCC & 4403150993428 & Domestic & 1,030 & Dakota \\
\hline Wesley Moore & 107-73-28BDAC & 4402430993550 & Unused & 1,240 & Dakota \\
\hline A. Zeigler & 107-73-29ABBC & 4402550993653 & Unused & 1,240 & Dakota \\
\hline Alfred Zeigler & 107-73-32CBCC & 4401310993730 & Domestic & 1,355 & Dakota \\
\hline Boyd Gourneau & 107-73-35BAAB & 4402070993326 & Stock & 1,385 & Dakota \\
\hline U.S. Army Corps of Engineers & 107-74- 3СВСВ & 4405550994217 & Public supply & 22 & Alluvium \\
\hline Lillian White & $107-74-5 \mathrm{ABCB}$ & 4406200994403 & Domestic & 6 & Alluvium \\
\hline George Christensen & 107-74-5ACDC & 4406040994356 & Domestic & 960 & Dakota \\
\hline Earl Christensen & 107-74-8CAAA & 4405100994410 & Domestic & 65 & Alluvium \\
\hline Merrill Karlen & $107-74-8 \mathrm{CA}$ & 4405040994416 & Unused & -- & -. \\
\hline Merrill Karlen & $107-74-8 \mathrm{CC}$ & 4404510994435 & Stock & 1,100 & Dakota \\
\hline Andrew Estes & 107-74-11AACB & 4405290994007 & Unused & 120 & Pierre \\
\hline Lower Brule Sioux Tribe & 107-74-19DAAA & 4403250994446 & Stock & -- & -- \\
\hline Berwin Svoboda & $107-74-23 \mathrm{AD}$ & 4403340994002 & -- & -- & -- \\
\hline B. Svoboda & 107-74-23ADDB & 4403320994002 & Stock & 1,270 & Dakota \\
\hline Frederick La Roche & 107-74-24AADC & 4403430993848 & Stock & 1,196 & $\begin{array}{c}\text { Dakota (and } \\
\text { Graneros }{ }^{2} \text { ) }\end{array}$ \\
\hline Jiggs Thompson & 107-74-24BA & 4403280993917 & Domestic & 1,180 & $\begin{array}{l}\text { Graneros (and } \\
\text { Dakota }^{2} \text { ) }\end{array}$ \\
\hline Jandreau brothers & 107-74-24CAAC & 4403220993925 & Stock & ${ }^{3} 1,100$ & $\begin{array}{l}\text { Dakota (and/or } \\
\text { Graneros }{ }^{2} \text { ) }\end{array}$ \\
\hline Jandreau brothers & 107-74-24CAAC2 & 4403220993925 & Stock & -- & Dakota \\
\hline Merrill Karlen & $107-74-25 \mathrm{AA}$ & 4402550993851 & Domestic & 1,147 & Graneros \\
\hline J.W. Thompson & 107-74-27CDDC & 4402100994150 & Stock & -- & Dakota $^{2}$ \\
\hline $\begin{array}{l}\text { Medicine Creek Grazing } \\
\text { Association }\end{array}$ & 107-74-31CBDC & 4401310994544 & Stock & ${ }^{3} 1,100$ & Dakota \\
\hline $\begin{array}{l}\text { Medicine Creek Grazing } \\
\text { Association }\end{array}$ & $107-74-32 C C D$ & 4401230994430 & Domestic & 1,100 & Dakota \\
\hline Jandreau brothers & $107-74-34 \mathrm{AD}$ & 4401490994116 & -- & 1,080 & Graneros \\
\hline $\begin{array}{l}\text { Medicine Creek Grazing } \\
\text { Association }\end{array}$ & 107-75-1AAAA & 4406260994557 & Stock & ${ }^{3} 1,000$ & $\begin{array}{l}\text { Dakota and/or } \\
\text { Graneros }\end{array}$ \\
\hline
\end{tabular}


Table 12. Usable wells on the Lower Brule Indian Reservation, 1992-Continued

\begin{tabular}{|c|c|c|c|c|c|}
\hline Owner & $\begin{array}{l}\text { Well number } \\
\text { (see page 10) }\end{array}$ & $\begin{array}{l}\text { Latitudel } \\
\text { longitude }\end{array}$ & $\begin{array}{l}\text { Primary use } \\
\text { of water } \\
\text { reported in } 1992\end{array}$ & $\begin{array}{l}\text { Depth } \\
\text { of well } \\
\text { (feet) }\end{array}$ & $\begin{array}{l}\text { Geologic unit(s) } \\
\text { in which well } \\
\text { is completed }\end{array}$ \\
\hline Larry Christian & 107-75- 4ADCA & 4406080994941 & Domestic & 1,326 & Dakota \\
\hline Larry Christian & 107-75- 4CACA & 4405550995019 & Stock & -- & Dakota or deeper \\
\hline $\begin{array}{l}\text { Alfred Andrew } \\
\text { Gus Allen }\end{array}$ & 107-75- 5ВСВA & 4406140995150 & Unused & 1,300 & Dakota \\
\hline Gus Allen & 107-75-7ADDB & 4405160995202 & Unused & 1,400 & Dakota \\
\hline Fay Jandreau & 107-75-11CAAA & 4405090994746 & Domestic & 1,400 & Dakota \\
\hline F. Jandreau & 107-75-12ADBB & 4405220994611 & Stock & 1,300 & Dakota \\
\hline Jerry Ireland & 107-75-16CDCA & 4403580995019 & -- & 1,500 & $\begin{array}{l}\text { Inyan Kara (and } \\
\text { Dakota }^{2} \text { ) }\end{array}$ \\
\hline Driving Hawk & $107-75-17 \mathrm{ABBC}$ & 4404410995110 & Domestic & 1,605 & Dakota \\
\hline Jim Schaefer & $107-75-20 \mathrm{BBBC}$ & 4403430995135 & Domestic & 1,260 & $\begin{array}{l}\text { Graneros (and } \\
\text { Dakota }^{2} \text { ) }\end{array}$ \\
\hline Jim Schaefer & 107-75-20ВСВ & 4403360995149 & Domestic & 1,600 & $\begin{array}{l}\text { Dakota and Inyan } \\
\text { Kara }\end{array}$ \\
\hline Smith and Smith & $107-75-29 \mathrm{CCCC}$ & 4402100995154 & Unused & 1,470 & Dakota \\
\hline Wayne Smith & 107-75-29CCDB & 4402150995200 & Domestic & -- & Dakota \\
\hline Keith Dorman & 107-75-30СBBD2 & 4402350995305 & Stock & 1,473 & Dakota \\
\hline Will Gorman & 107-75-32CCBD & 4401240995149 & -- & 1,430 & Dakota \\
\hline Mike Jandreau & 107-76- 4DCCD & 4405430995712 & Unused & 1,050 & Graneros \\
\hline Mike Jandreau & 107-76-9CDAC & 4404520995727 & Domestic & 1,367 & Dakota \\
\hline Mike Jandreau & 107-76- 9DDAB & 4405000995647 & Domestic & 1,335 & Dakota \\
\hline D. Gifford & 107-76-10AA & 4405300995540 & -- & 1,100 & $\begin{array}{l}\text { Dakota and/or } \\
\text { Graneros }\end{array}$ \\
\hline Sharon Brown & 107-76-10DD & 4404230995536 & Domestic & 1,410 & Dakota \\
\hline Dallas Gifford & $107-76-12 \mathrm{AD}$ & 4405170995316 & Unused & -- & Dakota $^{2}$ \\
\hline Roscoe Huston & $107-76-26 \mathrm{CBCB} 2$ & 4402330995530 & Domestic & 1,640 & Dakota \\
\hline R. Huston & $107-76-26 \mathrm{CCCC}$ & 4402090995529 & Stock & 1,440 & Dakota and Graneros \\
\hline R. Huston & 107-76-26CDCD & 4402090995506 & Unused & 1,400 & Graneros \\
\hline Huston brothers & $107-76-26 \mathrm{CDCD} 2$ & 4402100995507 & Domestic & 1,603 & Dakota \\
\hline Patricia Madsen & 107-77-13DAAC & 4404141000024 & Domestic & 1,400 & Dakota and Graneros \\
\hline Lower Brule & 108-73-5CDDD & 4411030993642 & Domestic & 90 & Alluvium \\
\hline J. Thompson & 108-73-10ВBВB & 4410470993503 & Domestic & 47 & Alluvium \\
\hline J.W. Thompson & 108-73-10ВBВB2 & 4410480993504 & -- & 1,030 & Dakota \\
\hline J. Thompson & 108-73-17ADBC & 4409390993638 & Stock & 980 & $\begin{array}{l}\text { Dakota and/or } \\
\text { Graneros }\end{array}$ \\
\hline Delmar Smith & 108-73-21AAAA & 4409030993510 & Irrigation & 1,200 & Dakota \\
\hline Lyle Peterson & 108-73-22BCCA & 4408440993500 & Domestic & 1,175 & Dakota \\
\hline U.S. Government & 108-73-28DDAD & 4407300993509 & Unused & -- & $\begin{array}{l}\text { Dakota and/or } \\
\text { Graneros }\end{array}$ \\
\hline Gillman Farms & 108-74-32DACD & 4406430994343 & Domestic & 47 & $\underset{\text { Pierre }^{2} \text { ) }}{\text { Alluvium² }}$ (or \\
\hline Lower Brule Sioux Tribe & $108-75-31 \mathrm{AC}$ & 4407020995223 & -- & -- & -- \\
\hline W.S. Christian & 108-75-34DCDD & 4406300994838 & Stock & 1,366 & Dakota \\
\hline Gillman Farms & 108-75-36BDCA & 4407000994644 & Stock & -- & -- \\
\hline
\end{tabular}


Table 12. Usable wells on the Lower Brule Indian Reservation, 1992-Continued

\begin{tabular}{|c|c|c|c|c|c|}
\hline Owner & $\begin{array}{l}\text { Well number } \\
\text { (see page 10) }\end{array}$ & $\begin{array}{l}\text { Latitude/ } \\
\text { longitude }\end{array}$ & $\begin{array}{l}\text { Primary use } \\
\text { of water } \\
\text { reported in } 1992\end{array}$ & $\begin{array}{l}\text { Depth } \\
\text { of well } \\
\text { (feet) }\end{array}$ & $\begin{array}{l}\text { Geologic unit(s) } \\
\text { in which well } \\
\text { is completed }^{1}\end{array}$ \\
\hline Gillman Farms & 108-75-36DDDD & 4406300994557 & Domestic & $>1,000$ & $\begin{array}{l}\text { Dakota (and/or } \\
\text { Graneros }{ }^{2} \text { ) }\end{array}$ \\
\hline Lloyd Langdeau & 108-76- 8AABC & 4410450995809 & Domestic & 1,550 & Dakota \\
\hline Abe Langdeau & 108-76- 8BBAD & 4410480995854 & Domestic & 1,430 & Dakota \\
\hline Lloyd Langdeau & 108-76- 9BBAB & 4410480995742 & Stock & -- & Dakota $^{2}$ \\
\hline Leslie Langdeau & 108-76-16CDCA & 4409110995729 & Domestic & ${ }^{3} 1,200$ & Dakota \\
\hline L. Langdeau & 108-76-16DD & 4409110995652 & Unused & 980 & $\begin{array}{l}\text { Graneros (and } \\
\text { Dakota }^{2} \text { ) }\end{array}$ \\
\hline Charles Langdeau & 108-76-21CCCD & 4408180995754 & Domestic & 1,200 & Dakota \\
\hline Vera Gandt & 108-76-21DCAD & 4408210995705 & Domestic & 1,100 & Dakota \\
\hline U.S. Army Corps of Engineers & 108-76-22ACDB & 4408440995557 & Public supply & 28 & Alluvium \\
\hline Pauline Middletent & 108-76-30ABAD & 4408060995930 & Domestic & -- & Dakota $^{2}$ \\
\hline J.B. Howe & $108-77-13 B$ & 4409451000114 & Unused & 1,450 & Dakota \\
\hline $\begin{array}{l}\text { Pat Durkin } \\
\text { Milton Ness }\end{array}$ & 108-77-14D & 4409141000143 & Stock & 1,890 & $\begin{array}{l}\text { Minnelusa, } \\
\text { Sundance, and } \\
\text { Inyan Kara }\end{array}$ \\
\hline A. Brakke & 108-77-22CBDD & 4408271000341 & Stock & 1,300 & Dakota \\
\hline Baunita and Bart Ness & 108-77-25BDCB & 4407531000114 & Stock & 1,300 & Dakota \\
\hline Andrew Brakke & 108-77-25BDCB2 & 4408231000332 & Stock & -- & $\begin{array}{l}\text { Graneros and/or } \\
\text { Dakota }^{2}\end{array}$ \\
\hline Baunita Ness & 108-77-25DABB & 4407461000036 & Domestic & 1,300 & Dakota \\
\hline Leslie N. Langdeau & 108-77-35DBAD & 4406501000153 & Unused & -- & Dakota $^{2}$ \\
\hline $\begin{array}{l}\text { Clark Ranch } \\
\text { Lower Brule Sioux Tribe }\end{array}$ & 109-76-10DBDA & 4415290995655 & Unused & -- & Dakota $^{2}$ \\
\hline $\begin{array}{l}\text { Clark Ranch } \\
\text { Lower Brule Sioux Tribe }\end{array}$ & 109-76-13ACCC & 4414460995444 & Unused & 80 & Alluvium \\
\hline Betty Durkin & $109-76-14 \mathrm{CCC}$ & 4413290995643 & Domestic & 1,093 & $\begin{array}{l}\text { Dakota (and/or } \\
\text { Graneros }{ }^{2} \text { ) }\end{array}$ \\
\hline $\begin{array}{l}\text { Clark Ranch } \\
\text { Lower Brule Sioux Tribe }\end{array}$ & 109-76-16ACDB & 4414490995810 & Unused & $>1,000$ & Dakota \\
\hline $\begin{array}{l}\text { Clark Ranch } \\
\text { Lower Brule Sioux Tribe }\end{array}$ & 109-76-16ACDB2 & 4414480995812 & Stock & $>1,000$ & Graneros \\
\hline Sally Durkin & 109-76-24BAD & 4414090995450 & Unused & 80 & Alluvium \\
\hline $\begin{array}{l}\text { Clark Ranch } \\
\text { Lower Brule Sioux Tribe }\end{array}$ & 109-76-26ABAC & 4413570995547 & Stock & 70 & Alluvium \\
\hline $\begin{array}{l}\text { Clark Ranch } \\
\text { Lower Brule Sioux Tribe }\end{array}$ & 109-76-26ACCB & 4413050995557 & Unused & 900 & Graneros \\
\hline $\begin{array}{l}\text { Clark Ranch } \\
\text { Lower Brule Sioux Tribe }\end{array}$ & 109-76-26BDBD & 4413080995611 & Stock & $>1,000$ & $\begin{array}{l}\text { Dakota (and } \\
\text { Graneros }^{2} \text { ) }\end{array}$ \\
\hline Bill Thompson & 109-77-2ADDD & 4416301000237 & Stock & 35 & Alluvium \\
\hline John Ketchen & $109-77-3 B$ & 4416391000513 & Stock & 1,655 & $\begin{array}{l}\text { Inyan Kara and } \\
\text { Sundance (and } \\
\text { Minnelusa } a^{2} \text { ) }\end{array}$ \\
\hline W. Thompson & 109-77-14CDDD & 4414201000315 & Stock & 1,400 & Dakota \\
\hline
\end{tabular}

${ }^{1}$ Determined from interpretation of well logs and/or structure-contour maps.

${ }^{2}$ Probable unit or possible alternative or additional unit.

${ }^{3}$ Depth estimated. 
Table 13.--Selected field analyses of ground-water samples collected in 1992 from wells on the Lower Brule Indian Reservation $\left[{ }^{\circ} \mathrm{C}\right.$, degree Celsius; $\mu \mathrm{S} / \mathrm{cm}$, microsiemens per centimeter at $25^{\circ} \mathrm{C} ; \mathrm{mg} / \mathrm{L}$, milligram per liter; --, not determined]

\begin{tabular}{|c|c|c|c|c|c|c|c|}
\hline Location & $\begin{array}{l}\text { Date } \\
\text { collected }\end{array}$ & $\begin{array}{c}\text { Geologic } \\
\text { unit }\end{array}$ & $\begin{array}{c}\text { Tempera- } \\
\text { ture } \\
\left({ }^{\circ} \mathrm{C}\right)\end{array}$ & $\begin{array}{c}\text { Specific } \\
\text { corductance } \\
(\mu \mathrm{S} / \mathrm{cm})\end{array}$ & pH & $\begin{array}{c}\text { Total } \\
\text { hardness } \\
\text { (mg/L) }\end{array}$ & $\begin{array}{c}\text { Chloride } \\
\text { (mg/L) }\end{array}$ \\
\hline 105-72- 5DADA & $9-16-92$ & Dakota & -- & 780 & 7.7 & 291 & 30 \\
\hline 105-72- 5DADD & $9-16-92$ & Dakota & -- & 2,350 & 7.9 & 1,215 & 91 \\
\hline 106-71-17DBAA & $9-16-92$ & Graneros (and Dakota ${ }^{1}$ ) & 7.0 & 2,360 & 7.6 & 130 & 152 \\
\hline 106-71-30СABA & $8-13-92$ & Dakota & 16.5 & 2,360 & 7.3 & 1,301 & 91 \\
\hline 106-71-32CCCD & $9-11-92$ & Dakotal & 15.5 & 2,630 & 7.7 & 1,507 & 91 \\
\hline 106-72-21CADC & $8-10-92$ & Dakota & 15.5 & 2,450 & 6.9 & 1,387 & 91 \\
\hline 106-72-26BCCA & $8-10-92$ & Dakota & 16.5 & 2,310 & 4.4 & 1,301 & 91 \\
\hline 106-72-28BDDD2 & $8-10-92$ & Dakota & 19.0 & 2,430 & 7.1 & 1,438 & 91 \\
\hline 106-72-33ADBC & $8-11-92$ & Dakota & 17 & 2,540 & 7.2 & 1,335 & 212 \\
\hline 107-73-22CCAC & $7-24-92$ & Unknown & 17.5 & 2,730 & 7.5 & 410 & 152 \\
\hline 107-73-22CCCC & $8-16-92$ & Dakota & -- & 2,550 & -- & -- & -- \\
\hline 107-73-22DBCC & $7-23-92$ & Dakota & 29.5 & 2,270 & 7.5 & 753 & 121 \\
\hline 107-74- 8CAAA & 8- $6-92$ & Alluvium & 16.5 & 1,730 & 7.4 & 685 & 61 \\
\hline 107-75- 4ADCA & $7-17-92$ & Dakota & 21.1 & 2,210 & 7.4 & 548 & 121 \\
\hline 107-75-20ВBВC & $7-20-92$ & Graneros (and Dakota') & 22.5 & 2,160 & 7.9 & 702 & 121 \\
\hline 107-75-29CCDB & $7-20-92$ & Dakota & 15.0 & 2,330 & 7.4 & 650 & 200 \\
\hline 107-76-9CDAC & $7-15-92$ & Dakota & 28.0 & 2,340 & 7.6 & 257 & 152 \\
\hline 108-76- 8АABC & $7-17-92$ & Dakota & 14.5 & 2,230 & 6.9 & -- & 334 \\
\hline 108-76-16CDCA & 7- 8-92 & Dakota & 26.2 & 2,260 & 7.4 & -- & 182 \\
\hline 108-76-21CCCD & 7- 8-92 & Dakota & 20.0 & 2,650 & 7.3 & 171 & 243 \\
\hline 108-76-21DCAD & 7- $2-92$ & Dakota & 19.5 & 2,450 & 7.5 & -- & 152 \\
\hline 108-76-30ABAD & $8-24-92$ & Dakota1 & 21.2 & 2,230 & 7.8 & 223 & 152 \\
\hline 108-77-25DABB & 7- $8-92$ & Dakota & 18.3 & 2,200 & 7.5 & -- & 182 \\
\hline
\end{tabular}

${ }^{1}$ Probable unit or possible alternative or additional unit.

declared ownership of ground water or surface water, nor with the State's authority to control the development or use of either ground-water or surface-water resources within the reservation (M.B. Jandreau, Tribal Chairman, Lower Brule Sioux Tribe, oral commun., February 1995).

The Water Rights Division of the South Dakota Department of Environment and Natural Resources (DENR) has issued several water rights (water use) permits (non-tribal) on deeded land within the Lower Brule Indian Reservation boundaries. When a State water right application is made, the Office of the Solicitor, U.S. Department of the Interior, on behalf of the Lower Brule Sioux Tribe, normally sends a letter of disclaimer to the DENR stating their objection to the issuance of a permit. The letter also states that by filing each objection, they do not waive their right to challenge State jurisdiction in the matter (J.A. Goodman, DENR, written commun., February 1995).

Permittees are required to report annually the amount of water used for the use specified in the permit. These annual-use values are maintained by the DENR in a data base. Because no permits are issued by the State for tribal entities, no values are reported to the DENR by the Lower Brule Sioux Tribe. 


\section{WATER USE}

A public water system supplies Missouri River water to the towns of Lower Brule and West Brule and to residences along the highway that connects these towns. The average usage during 1992 was 130,000 to 140,000 gallons per day, based on records maintained by the manager of the system. The primary uses of ground water on the reservation were given in table 12; however, the amount of use is not known. Irrigation constitutes most of the water use on the Lower Brule Reservation. In 1990, slightly more than 17,000 acre-feet was used to irrigate land within the reservation (table 14). Usage data for various water-use categories are maintained by the USGS in its Water-Use Data System (WUDS).

\section{SUMMARY}

The Lower Brule Indian Reservation covers an area of about 404 square miles, about 35 square miles of which are covered by major reservoirs on the Missouri River. Of the remaining 369 square miles of land, about 201 square miles are tribal and other Indian-owned land. Agriculture, primarily livestock production and crop production, is the major industry. In 1992, about 5,900 acres were irrigated by the Tribe. In addition, there were about 3,800 acres of other land within the reservation boundary either under irrigation or for which State permits for irrigation had been issued.

Inflow of the Missouri River to the Lower Brule Indian Reservation during 1968-92 is estimated to have averaged 18,290,000 acre-feet per year. Average runoff to the Missouri River in the reach adjacent to the reservation is estimated to be 64,000 acre feet per year. Diversions from the Missouri River within the reach are estimated to be 43,000 acre feet (based on 1990 usage). Net evaporation from the Missouri River reservoirs (Lakes Sharpe and Frances Case) in the reach adjacent to the reservation is estimated to average 146,000 acre-feet per year. From this, average outflow of the Missouri River from the reservation is estimated to be $18,165,000$ acre-feet per year. Lakes Sharpe and Francis Case normally store about 5 million acre-feet of water. Precipitation on the land area of the reservation averages about 17.4 inches per year. Evapotranspiration from the land surface of the reservation averages about 17.5 inches.

Upstream reservoirs on the Missouri River normally store about 51.9 million acre-feet and stabilize water quality. Dissolved-solids concentration of Missouri River reservoir water available to the reservation averages about $500 \mathrm{mg} / \mathrm{L}$. The total discharge to the Missouri River reservoirs from all streams on the reservation, which are ephemeral, averages about 26,000 acre-feet per year. The USGS water-quality data base contains data for two samples taken from streams in 1971.

Stock ponds and dugouts are a major source of water for livestock on the reservation. In 1993, 593 stock ponds and dugouts, with a total area of more than 1,300 acres, were in use or were in repairable condition. Almost all such impoundments go dry by late summer and during droughts. Based on visits to about 10 percent of the ponds, approximately 75 percent were dry, overgrown with vegetation, badly silted in, had breached or leaky dams, or were otherwise not in good condition. Like streamflow, the quality of water in stock ponds probably varies widely during the year, depending on dilution by runoff, concentration of salts by evaporation, and influshing of soluble minerals and organic matter.

Shallow ground-water supplies are limited to alluvial deposits along major streams and to terrace deposits where they extend below the water level of the Missouri River reservoirs. Where such water supplies have been in contact with the Pierre Shale, the dissolved-solids concentration can exceed $4,000 \mathrm{mg} / \mathrm{L}$.

Most ground water on the Lower Brule Reservation is obtained from deep, artesian aquifers in the bedrock, usually the Dakota Sandstone, although a few wells tap deeper aquifers. Dissolved-solids concentrations in water from the Dakota Sandstone commonly are from 2,000 to $3,000 \mathrm{mg} / \mathrm{L}$; the water is of sodium chloride, sodium sulfate, or calcium magnesium sulfate type. From the well inventory, 139 wells in usable condition were identified; of these, 101 were in use.

Federal law and court decisions support the concept that Indian reservations have reserved for 


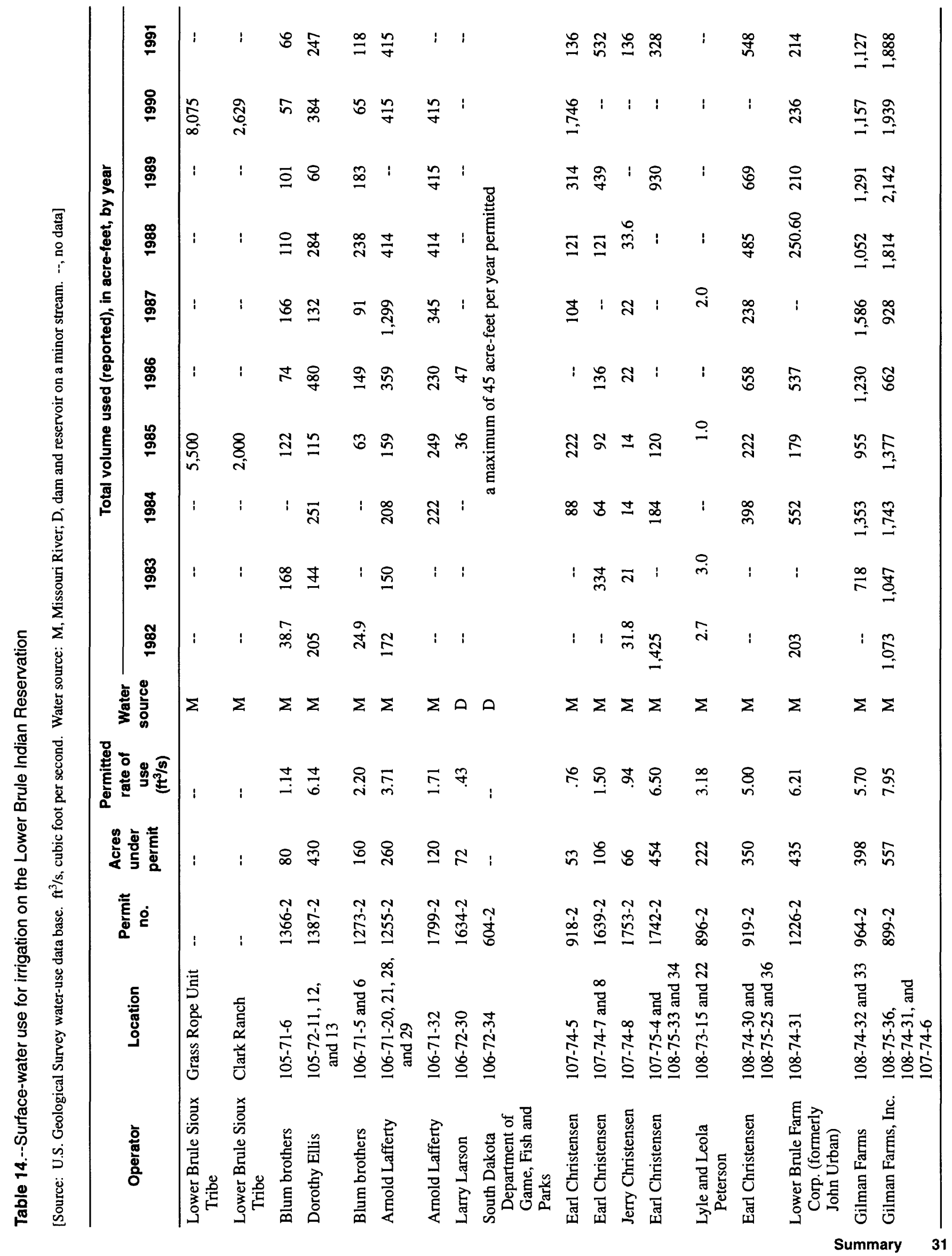


them all the water needed to make the land productive, including water sufficient to irrigate all potentially arable land. In South Dakota, the State claims ownership of the ground and surface water for the purpose of regulating the development and use of such water. Indian tribes, including the Lower Brule Sioux Tribe, do not necessarily concur that the State owns or controls water within or adjacent to reservation boundaries (in this case, the Missouri River).

Irrigation constitutes most of the water use on the Lower Brule Reservation. In 1990, slightly more than 17,000 acre-feet was used to irrigate land within the reservation.

\section{REFERENCES CITED}

Becker, L.D., 1974, A method for estimating magnitude and frequency of floods in South Dakota: U.S. Geological Survey Water-Resources Investigations Report 35-74, $78 \mathrm{p}$.

-1980, Techniques for estimating flood peaks, volumes, and hydrographs on small streams in South Dakota: U.S. Geological Survey Water-Resources Investigations Report 80-80, 82 p.

Bureau of Indian Affairs, 1964, Soil and range resources inventory, Lower Brule Indian Reservation, South Dakota: Missouri River Basin Investigation Project, Report 176, 67 p.

Bureau of Reclamation, 1979, Proposed feasibility report on Grass Rope Unit: Missouri-Oahe Projects Office, Huron, South Dakota, [about 110 pages].

Farnsworth, R.K., Thompson, E.S., and Peck, E.L., 1982, Evaporation atlas for the contiguous 48 United States: National Oceanic and Atmospheric Administration Technical Report NWS 33, 26 p.

Foster, K.E., 1978, The Winters Doctrine: Historical perspective and future applications of reserved water rights in Arizona: Ground Water, v. 16, no. 3, p. 186191.

Francis-Meador-Gellhaus, Inc., Consulting Engineers, 1978, Water resource inventory, Lower Brule Indian Reservation, Lower Brule, South Dakota: Phase I, The water resource base, $54 \mathrm{p}$.

-1980, A water resource inventory of the Lower Brule Indian Reservation: Phase II, 92 p.

Helsel, D.R., and Gilliom, R.J., 1985, Estimation of distributional parameters for censored trace-level waterquality data; II, Verification and applications: U.S. Geological Survey Open-File Report 85-86, 21 p.

Howells, L.W., 1974, Geohydrology of Crow Creek and Lower Brule Indian Reservations, South Dakota: U.S. Geological Survey Hydrologic Investigations Atlas HA-499, 2 sheets.

La Rocque, G.A., Jr., 1966, General availability of ground water and depth to water level in the Missouri River Basin: U.S. Geological Survey Hydrologic Investigations Atlas HA-217, 2 sheets, scale 1:2,500,000.

Larimer, O.J., 1970, A proposed streamflow data program for South Dakota: U.S. Geological Survey Open-file Report 70-194, 60 p.

U.S. Army Corps of Engineers, 1993, 1993-1994 Missouri River main stem reservoirs annual operating plan and summary of actual 1992-1993 operations: U.S. Army Corps of Engineers, Missouri River Division, Reservoir Control Center, Omaha, Nebr., 124 p.

U.S. Environmental Protection Agency, 1993, Summary of EPA finalized National primary drinking water regulations: U.S. Environmental Protection Agency Region VIII.

Winter, D.R., 1994, Water levels in bedrock aquifers in South Dakota: U.S. Geological Survey Open-File Report 94-320, 279 p. 
SUPPLEMENTAL INFORMATION 
Table 15. Daily discharge for the Missouri River at Pierre (station 06440000) for water years 1934-65

[---, no data; MAX, maximum value; MIN, minimum value; AC-FT, acre-feet $]$

\begin{tabular}{|c|c|c|c|c|c|c|c|c|c|c|c|c|}
\hline Day & Oct. & Nov. & Dec. & Jan. & Feb. & Mar. & April & May & June & July & Aug. & Sept. \\
\hline \multicolumn{13}{|c|}{$\begin{array}{c}\text { DISCHARGE, CUBIC FEET PER SECOND, WATER YEAR OCTOBER } 1933 \text { TO SEPTEMBER } 1934 \\
\text { DAILY MEAN VALUES }\end{array}$} \\
\hline 1 & 10,800 & 9,880 & 13,400 & 3,800 & 11,200 & 33,400 & 19,500 & 20,000 & 24,500 & 26,200 & 8,550 & 5,050 \\
\hline 2 & 10,900 & 9,920 & 13,500 & 4,200 & 10,800 & 23,700 & 18,400 & 20,000 & 25,300 & 24,500 & 8,150 & 4,800 \\
\hline 3 & 10,700 & 10,500 & 13,400 & 4,500 & 10,600 & 22,400 & 19,500 & 19,500 & 24,500 & 23,700 & 7,850 & 4,600 \\
\hline 4 & 10,500 & 11,000 & 13,000 & 4,600 & 10,600 & 21,200 & 20,000 & 20,000 & 23,700 & 22,400 & 7,530 & 4,500 \\
\hline 5 & 10,300 & 11,000 & $\mathrm{I} 2,500$ & 4,600 & 10,600 & 19,000 & 19,500 & 20,600 & 23,700 & 21,800 & 6,950 & 4,600 \\
\hline 6 & 10,000 & 11,100 & 12,200 & 4,800 & 10,600 & 19,500 & 18,400 & 23,000 & 23,000 & 21,200 & 6,950 & 4,500 \\
\hline 7 & 9,880 & 11,000 & 11,900 & 5,000 & 10,700 & 19,500 & 18,400 & 29,700 & 23,000 & 19,500 & 6,900 & 4,400 \\
\hline 8 & 9,800 & 10,300 & 12,800 & 5,200 & 10,700 & 16,100 & 26,200 & 31,500 & 21,800 & 18,400 & 6,900 & 4,400 \\
\hline 9 & 9,680 & 9,150 & 12,000 & 5,300 & 10,800 & 16,100 & 24,500 & 28,800 & 21,200 & 17,500 & 6,800 & 4,400 \\
\hline 10 & 9,680 & 7,220 & 10,000 & 5,400 & 11,700 & 17,500 & 20,000 & 26,200 & 21,800 & 17,000 & 7,050 & 4,400 \\
\hline 11 & 9,720 & 7,040 & 9,400 & 5,700 & 12,500 & 16,100 & 17,500 & 26,200 & 22,400 & 16,500 & 9,250 & 4,400 \\
\hline 12 & 9,680 & 6,380 & 8,000 & 6,000 & 13,000 & 17,500 & 16,100 & 25,300 & 23,000 & 15,600 & 9,950 & 4,500 \\
\hline 13 & 9,840 & 6,110 & 7,400 & 6,300 & 13,600 & 17,000 & 18,400 & 24,500 & 22,400 & 15,200 & 9,950 & 4,500 \\
\hline 14 & 9,680 & 6,520 & 7,000 & 6,700 & 14,300 & 17,500 & 21,200 & 23,000 & 21,200 & 13,800 & 9,250 & 4,500 \\
\hline 15 & 9,640 & 8,860 & 6,600 & 7,000 & 15,800 & 17,900 & 21,200 & 23,000 & 20,600 & 13,400 & 8,900 & 4,600 \\
\hline 16 & 9,640 & 10,700 & 6,400 & 7,400 & 19,300 & 18,400 & 19,500 & 23,000 & 21,200 & 12,900 & 9,250 & 4,600 \\
\hline 17 & 9,600 & 8,720 & 6,100 & 7,700 & 18,300 & 23,000 & 18,400 & 22,400 & 29,700 & 12,500 & 8,900 & 4,700 \\
\hline 18 & 9,640 & 20,800 & 5,900 & 8,000 & 17,800 & 21,200 & 17,500 & 26,200 & 34,400 & 12,100 & 8,250 & 4,900 \\
\hline 19 & 9,680 & 25,500 & 5,600 & 8,200 & 17,900 & 16,500 & 17,000 & 30,600 & 44,600 & 11,700 & 7,350 & 4,900 \\
\hline 20 & 9,680 & 20,900 & 5,400 & 8,300 & 17,000 & 17,500 & 17,000 & 31,500 & 47,600 & 11,200 & 7,050 & 4,900 \\
\hline 21 & 9,330 & 17,100 & 5,100 & 8,500 & 15,000 & 20,600 & 17,000 & 28,800 & 41,800 & 10,800 & 6,500 & 5,000 \\
\hline 22 & 9,260 & 15,400 & 4,900 & 8,600 & 13,800 & 37,700 & 17,000 & 27,000 & 32,400 & 10,800 & 6,250 & 5,100 \\
\hline 23 & 9,450 & 14,500 & 4,700 & 8,800 & 12,800 & 40,400 & 17,900 & 26,200 & 27,900 & 10,000 & 6,000 & 5,100 \\
\hline 24 & 9,220 & 14,400 & 4,500 & 8,900 & 10,600 & 27,900 & 17,900 & 26,200 & 27,900 & 9,620 & 5,950 & 5,000 \\
\hline 25 & 9,370 & 14,600 & 4,200 & 9,100 & 9,600 & 23,000 & 18,400 & 26,200 & 29,700 & 10,000 & 6,050 & 5,000 \\
\hline 26 & 9,410 & 14,700 & 4,000 & 9,300 & 8,700 & 21,800 & 18,400 & 24,500 & 30,600 & 9,620 & 5,700 & 4,900 \\
\hline 27 & 9,520 & 15,100 & 3,900 & 9,900 & 8,600 & 20,600 & 19,000 & 23,700 & 31,500 & 8,890 & 5,700 & 5,000 \\
\hline 28 & 9,760 & 14,600 & 3,800 & 10,200 & 8,700 & 17,900 & 19,500 & 23,000 & 30,600 & 8,890 & 5,500 & 5,000 \\
\hline 29 & 9,560 & 14,000 & 3,800 & 10,800 & --- & 19,000 & 19,500 & 23,000 & 28,800 & 9,620 & 5,500 & 5,100 \\
\hline 30 & 9,490 & 13,800 & 3,800 & 11,200 & -- & 17,900 & 20,000 & 23,000 & 27,000 & 10,000 & 5,250 & 5,200 \\
\hline 31 & 9,640 & -- & 3,800 & 11,300 & -- & 18,400 & --- & 23,700 & -- & 8,890 & 5,250 & -- \\
\hline TOTAL & 303,050 & 370,800 & 239,000 & 225,300 & 355,600 & 656,200 & 572,800 & 770,300 & 827,800 & 454,230 & 225,380 & 142,550 \\
\hline MEAN & 9,776 & 12,360 & 7,710 & 7,268 & 12,700 & 21,170 & 19,090 & 24,850 & 27,590 & 14,650 & 7,270 & 4,752 \\
\hline MAX & 10,900 & 25,500 & 13,500 & 11,300 & 19,300 & 40,400 & 26,200 & 31,500 & 47,600 & 26,200 & 9,950 & 5,200 \\
\hline MIN & 9,220 & 6,110 & 3,800 & 3,800 & 8,600 & 16,100 & 16,100 & 19,500 & 20,600 & 8,890 & 5,250 & 4,400 \\
\hline AC-FT & 601,100 & 735,500 & 474,100 & 446,900 & 705,300 & $1,302,000$ & $1,136,000$ & $1,528,000$ & $1,642,000$ & 901,000 & 447,000 & 282,700 \\
\hline
\end{tabular}


Table 15. Daily discharge for the Missouri River at Pierre (station 06440000) for water years 1934-65—Continued [---, no data; MAX, maximum value; MIN, minimum value; AC-FT, acre-feet $]$

\begin{tabular}{|c|c|c|c|c|c|c|c|c|c|c|c|c|}
\hline Day & Oct. & Nov. & Dec. & Jan. & Feb. & Mar. & April & May & June & July & Aug. & Sept. \\
\hline \multicolumn{13}{|c|}{$\begin{array}{c}\text { DISCHARGE, CUBIC FEET PER SECOND, WATER YEAR OCTOBER } 1934 \text { TO SEPTEMBER } 1935 \\
\text { DAILY MEAN VALUES }\end{array}$} \\
\hline 1 & 5,250 & 8,100 & 8,300 & 5,300 & 5,400 & 10,600 & 13,200 & 17,600 & 20,700 & 60,200 & 23,900 & 11,000 \\
\hline 2 & 5,250 & 8,340 & 8,000 & 5,600 & 5,800 & 10,400 & 13,500 & 16,300 & 38,900 & 61,200 & 24,200 & 10,600 \\
\hline 3 & 5,250 & 8,250 & 6,600 & 6,000 & 6,800 & 10,300 & 15,000 & 15,900 & 77,400 & 55,200 & 23,900 & 10,100 \\
\hline 4 & 5,500 & 8,370 & 5,400 & 6,000 & 7,000 & 10,100 & 15,600 & 16,500 & 65,800 & 48,000 & 23,900 & 9,600 \\
\hline 5 & 5,500 & 8,250 & 4,500 & 5,900 & 6,600 & 10,000 & 16,000 & 17,800 & 60,200 & 43,400 & 23,200 & 9,600 \\
\hline 6 & 5,500 & 8,550 & 3,600 & 5,800 & 7,100 & 9,800 & 17,700 & 18,400 & 48,800 & 43,400 & 22,500 & 9,580 \\
\hline 7 & 6,000 & 8,550 & 3,800 & 5,800 & 7,600 & 9,700 & 19,600 & 20,000 & 43,400 & 42,700 & 20,600 & 9,120 \\
\hline 8 & 6,000 & 9,250 & 4,000 & 5,800 & 8,400 & 9,700 & 20,200 & 19,700 & 40,000 & 45,600 & 19,400 & 8,790 \\
\hline 9 & 5,950 & 9,250 & 4,500 & 5,800 & 8,500 & 9,800 & 21,200 & 19,800 & 48,800 & 49,600 & 18,600 & 8,420 \\
\hline 10 & 6,000 & 9,250 & 5,200 & 5,800 & 7,500 & 10,000 & 23,200 & 20,100 & 50,500 & 48,800 & 17,000 & 8,350 \\
\hline 11 & 6,450 & 9,250 & 5,400 & 5,800 & 6,500 & 10,200 & 24,000 & 19,900 & 44,900 & 43,400 & 1,5600 & 8,100 \\
\hline 12 & 6,450 & 9,250 & 5,200 & 5,800 & 6,000 & 10,500 & 24,600 & 19,400 & 39,300 & 46,400 & 14,600 & 8,000 \\
\hline 13 & 6,950 & 9,250 & 4,400 & 5,500 & 5,400 & 10,700 & 25,600 & 18,900 & 35,200 & 79,800 & 13,600 & 7,750 \\
\hline 14 & 7,250 & 9,250 & 4,800 & 5,300 & 6,000 & 11,100 & 26,600 & 18,400 & 33,300 & 127,000 & 12,400 & 7,600 \\
\hline 15 & 7,550 & 9,250 & 4,900 & 5,000 & 6,500 & 11,500 & 34,300 & 17,900 & 35,800 & 100,000 & 11,800 & 7,800 \\
\hline 16 & 8,150 & 9,250 & 5,100 & 4,800 & 7,500 & 11,800 & 38,400 & 16,800 & 37,000 & 62,300 & 11,400 & 7,800 \\
\hline 17 & 8,450 & 9,250 & 5,300 & 4,500 & 9,000 & 12,300 & 37,400 & 17,500 & 54,200 & 49,600 & 11,000 & 7,950 \\
\hline 18 & 8,450 & 9.250 & 5,400 & 4,300 & 10,300 & 12,700 & 30,000 & 17,300 & 67,800 & 42,000 & 10,600 & 8,100 \\
\hline 19 & 8,880 & 9,250 & 5,500 & 4,100 & 11,000 & 13,700 & 19,900 & 16,700 & 82,400 & 34,300 & 10,400 & 8,400 \\
\hline 20 & 10,200 & 9,250 & 6,000 & 3,900 & 11,800 & 14,000 & 15,800 & 16,300 & 89,000 & 30,600 & 10,300 & 8,400 \\
\hline 21 & 10,600 & 9,250 & 5,500 & 3,900 & 11,600 & 14,500 & 14,700 & 18,100 & 82,400 & 28,000 & 10,100 & 8,400 \\
\hline 22 & 9,950 & 9,250 & 5,500 & 3,900 & 11,200 & 14,300 & 14,100 & 22,300 & 79,800 & 26,400 & 9,950 & 8,100 \\
\hline 23 & 9,900 & 9,250 & 5,000 & 4,000 & 10,400 & 13,900 & 13,700 & 18,700 & 82,100 & 25,000 & 9,420 & 7,950 \\
\hline 24 & 9,700 & 9,250 & 4,400 & 4,000 & 9,800 & 13,300 & 13,700 & 18,400 & 78,600 & 24,600 & 8,900 & 7,950 \\
\hline 25 & 9,340 & 9,250 & 4,300 & 4,000 & 10,000 & 12,600 & 14,500 & 17,700 & 73,700 & 24,600 & 8,720 & 7,950 \\
\hline 26 & 8,970 & 8,900 & 4,200 & 4,000 & 10,200 & 12,800 & 18,200 & 16,700 & 64,600 & 26,000 & 8,550 & 7,750 \\
\hline 27 & 8,970 & 8,900 & 4,000 & 4,000 & 10,400 & 13,900 & 19,300 & 16,100 & 59,200 & 25,000 & 8,550 & 7,590 \\
\hline 28 & 8,340 & 8,900 & 4,200 & 4,100 & 10,500 & 13,700 & 20,400 & 15,700 & 57,200 & 23,600 & 8,400 & 7,500 \\
\hline 29 & 8,340 & 8,800 & 4,400 & 4,400 & --- & 13,400 & 18,200 & 15,500 & 58,200 & 23,200 & 8,550 & 7,350 \\
\hline 30 & 8,340 & 8,700 & 4,600 & 4,600 & -- & 13,400 & 18,200 & 15,000 & 57,200 & 22,500 & 8,900 & 7,200 \\
\hline 31 & 8,340 & -- & 4,900 & 5,000 & --- & 13,300 & --- & 14,800 & -.. & 22,800 & 10,300 & --- \\
\hline TOTAL & 235,770 & 269,110 & 156,900 & 152,700 & 234,800 & 368,000 & 616,800 & 550,200 & $1,706,400$ & $1,385,200$ & 439,240 & 252,800 \\
\hline MEAN & 7,605 & 8,970 & 5,061 & 4,926 & 8,386 & 11,870 & 20,560 & 17,750 & 56,880 & 44,680 & 14,170 & 8,427 \\
\hline MAX & 10,600 & 9,250 & 8,300 & 6,000 & 11,800 & 14,500 & 38,400 & 22,300 & 89,000 & 127,000 & 24,200 & 11,000 \\
\hline MIN & 5,250 & 8,100 & 3,600 & 3,900 & 5,400 & 9,700 & 13,200 & 14,800 & 20,700 & 22,500 & 8,400 & 7,200 \\
\hline AC-FT & 467,600 & 533,800 & 311,200 & 302,900 & 465,700 & 729,900 & $1,223,000$ & $1,091,000$ & $3,385,000$ & $2,748,000$ & 871,200 & 501,400 \\
\hline
\end{tabular}


Table 15. Daily discharge for the Missouri River at Pierre (station 06440000) for water years 1934-65—Continued [---, no data; MAX, maximum value; MIN, minimum value; AC-FT, acre-feet $]$

\begin{tabular}{|c|c|c|c|c|c|c|c|c|c|c|c|c|}
\hline Day & Oct. & Nov. & Dec. & Jan. & Feb. & Mar. & April & May & June & Juiy & Aug. & Sept. \\
\hline \multicolumn{13}{|c|}{$\begin{array}{c}\text { DISCHARGE, CUBIC FEET PER SECOND, WATER YEAR OCTOBER } 1935 \text { TO SEPTEMBER } 1936 \\
\text { DAILY MEAN VALUES }\end{array}$} \\
\hline 1 & 7,500 & 8,840 & 6,900 & 6,800 & 6,200 & 5,000 & 19,400 & 23,200 & 33,600 & 29,600 & 13,600 & 9,950 \\
\hline 2 & 7,400 & 8,720 & 7,100 & 6,600 & 6,300 & 5,500 & 18,800 & 22,700 & 32,400 & 28,800 & 13,000 & 9,950 \\
\hline 3 & 7,400 & 8,700 & 7,200 & 6,300 & 6,500 & 6,500 & 17,800 & 22,200 & 32,000 & 28,000 & 12,400 & 9,950 \\
\hline 4 & 7,400 & 8,500 & 7,300 & 6,000 & 6,400 & 7,500 & 17,300 & 21,500 & 31,600 & 27,600 & 11,800 & 10,200 \\
\hline 5 & 7,500 & 8,300 & 7,400 & 5,700 & 6,300 & 8,500 & 16,200 & 21,800 & 30,200 & 26,000 & 11,000 & 9,680 \\
\hline 6 & 7,480 & 7,700 & 7,500 & 5,600 & 6,200 & 10,000 & 15,200 & 22,800 & 31,000 & 25,300 & 10,400 & 9,600 \\
\hline 7 & 7,330 & 6,900 & 7,600 & 5,500 & 6,200 & 13,000 & 14,600 & 23,200 & 36,400 & 24,200 & 10,100 & 9,420 \\
\hline 8 & 7,350 & 5,900 & 7,700 & 5,300 & 6,000 & 18,000 & 13,800 & 22,500 & 45,600 & 23,200 & 9,780 & 9,080 \\
\hline 9 & 7,350 & 5,200 & 7,800 & 5,200 & 5,900 & 22,200 & 13,400 & 21,800 & 67,700 & 23,200 & 9,600 & 8,720 \\
\hline 10 & 7,400 & 4,800 & 7,900 & 5,200 & 5,900 & 23,000 & 13,400 & 21,200 & 63,800 & 22,500 & 9,600 & 8,250 \\
\hline 11 & 7,200 & 4,400 & 8,000 & 5,200 & 5,900 & 24,000 & 14,400 & 20,300 & 48,700 & 20,900 & 9,250 & 8,100 \\
\hline 12 & 7,400 & 4,200 & 8,200 & 5,200 & 5,900 & 23,000 & 16,200 & 19,400 & 42,100 & 20,600 & 9,080 & 7,950 \\
\hline 13 & 7,550 & 3,800 & 8,400 & 5,200 & 5,900 & 23,600 & 16,200 & 18,800 & 37,300 & 18,800 & 8,900 & 7,800 \\
\hline 14 & 7,710 & 3,700 & 8,500 & 5,200 & 5,900 & 23,600 & 18,800 & 18,800 & 38,200 & 18,300 & 8,900 & 7,500 \\
\hline 15 & 7,870 & 3,700 & 8,600 & 5,300 & 5,900 & 23,500 & 37,600 & 18,800 & 47,200 & 17,600 & 8,720 & 7,200 \\
\hline 16 & 7,870 & 3,700 & 8,700 & 5,400 & 6,000 & 22,700 & 75,000 & 21,800 & 47,200 & 16,600 & 8,550 & 7,200 \\
\hline 17 & 8,030 & 3,700 & 8,700 & 5,500 & 6,100 & 22,600 & 82,400 & 28,000 & 44,100 & 16,200 & 8,900 & 7,250 \\
\hline 18 & 8,190 & 3,800 & 8,600 & 5,600 & 6,200 & 24,600 & 51,400 & 29,600 & 40,600 & 15,400 & 9,950 & 7,240 \\
\hline 19 & 7,830 & 4,000 & 8,400 & 5,700 & 6,300 & 86,300 & 41,300 & 29,200 & 37,100 & 15,000 & 11,400 & 7,560 \\
\hline 20 & 8,030 & 4,300 & 8,200 & 6,000 & 6,300 & 54,200 & 35,800 & 28,400 & 35,100 & 14,800 & 12,400 & 7,650 \\
\hline 21 & 7,870 & 4,500 & 8,000 & 6,100 & 6,200 & 43,400 & 32,800 & 27,600 & 34,800 & 15,000 & 12,400 & 7,750 \\
\hline 22 & 8,190 & 4,800 & 7,800 & 6,200 & 6,000 & 37,600 & 27,200 & 35,800 & 34,800 & 15,600 & 12,000 & 7,750 \\
\hline 23 & 8,350 & 5,100 & 7,600 & 6,200 & 5,800 & 54,200 & 23,600 & 59,200 & 38,200 & 16,400 & 11,600 & 7,600 \\
\hline 24 & 8,350 & 5,300 & 7,400 & 6,100 & 5,700 & 49,600 & 21,500 & 52,300 & 40,600 & 15,900 & 11,000 & 7,650 \\
\hline 25 & 8,350 & 5,500 & 7,200 & 5,900 & 5,400 & 44,100 & 21,400 & 45,000 & 40,600 & 15,200 & 10,600 & 7,500 \\
\hline 26 & 8,350 & 5,700 & 7,000 & 5,700 & 5,200 & 31,900 & 21,900 & 38,600 & 40,000 & 15,200 & 10,400 & 7,350 \\
\hline 27 & 8,350 & 5,900 & 6,800 & 5,500 & 4,900 & 27,600 & 21,300 & 35,200 & 38,200 & 15,900 & 10,600 & 7,350 \\
\hline 28 & 8,110 & 6,200 & 6,700 & 5,400 & 4,600 & 27,200 & 21,800 & 33,600 & 35,800 & 16,400 & 11,600 & 7,200 \\
\hline 29 & 8,500 & 6,400 & 6,700 & 5,500 & 4,500 & 26,800 & 22,800 & 34,800 & 32,800 & 16,600 & 11,600 & 7,200 \\
\hline 30 & 8,660 & 6,700 & 6,900 & 5,700 & --- & 22,200 & 23,800 & 35,300 & 31,000 & 15,900 & 10,800 & 7,350 \\
\hline 31 & 8,760 & --- & 6,900 & 5,900 & $\ldots$ & 19,700 & -- & 35,300 & -.. & 14,400 & 10,400 & -- \\
\hline TOTAL & 243,630 & 168,960 & 237,700 & 176,700 & 170,600 & 831,600 & 787,100 & 888,700 & $1,188,700$ & 605,100 & 330,330 & 244,950 \\
\hline MEAN & 7,859 & 5,632 & 7,668 & 5,700 & 5,883 & 26,830 & 26,240 & 28,670 & 39,620 & 19,520 & 10,660 & 8,165 \\
\hline MAX & 8,760 & 8,840 & 8,700 & 6,800 & 6,500 & 86,300 & 82,400 & 59,200 & 67,700 & 29,600 & 13,600 & 10,200 \\
\hline MIN & 7,200 & 3,700 & 6,700 & 5,200 & 4,500 & 5,000 & 13,400 & 18,800 & 30,200 & 14,400 & 8,550 & 7,200 \\
\hline AC-FT & 483,200 & 335,100 & 471,500 & 350,500 & 338,400 & $1,649,000$ & $1,561,000$ & $1,763,000$ & $2,358,000$ & $1,200,000$ & 655,200 & 485,900 \\
\hline
\end{tabular}


Table 15. Daily discharge for the Missouri River at Pierre (station 06440000) for water years 1934-65-Continued [--, no data; MAX, maximum value; MIN, minimum value; AC-FT, acre-feet $]$

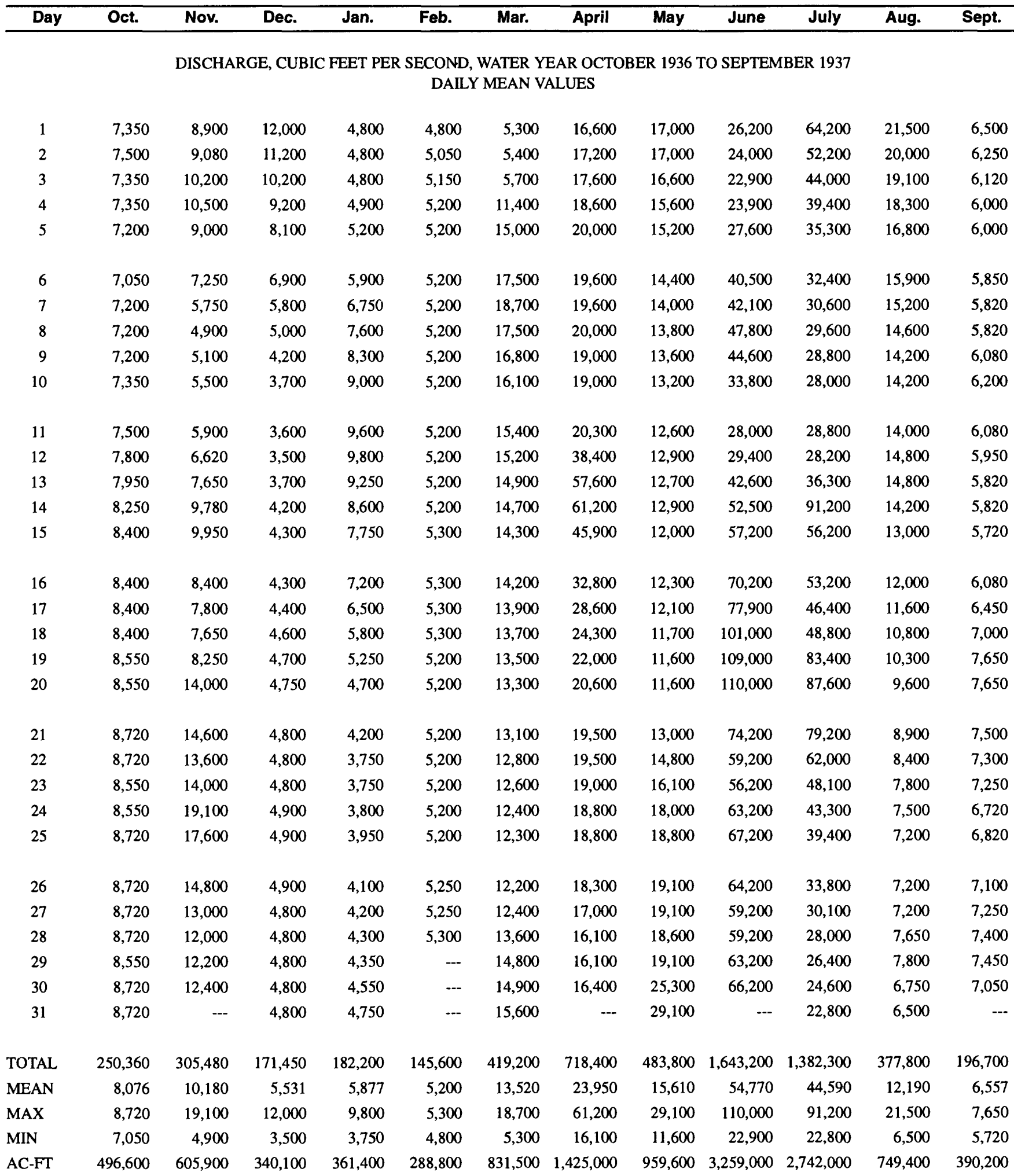


Table 15. Daily discharge for the Missouri River at Pierre (station 06440000) for water years 1934-65—Continued [---, no data; MAX, maximum value; MIN, minimum value; AC-FT, acre-feet]

\begin{tabular}{|c|c|c|c|c|c|c|c|c|c|c|c|c|}
\hline Day & Oct. & Nov. & Dec. & Jan. & Feb. & Mar. & April & May & June & July & Aug. & Sept. \\
\hline \multicolumn{13}{|c|}{$\begin{array}{c}\text { DISCHARGE, CUBIC FEET PER SECOND, WATER YEAR OCTOBER } 1937 \text { TO SEPTEMBER } 1938 \\
\text { DAILY MEAN VALUES }\end{array}$} \\
\hline 1 & 6,750 & 10,200 & 5,380 & 5,200 & 6,700 & 7,600 & 25,300 & 11,900 & 23,200 & 96,100 & 36,400 & 19,700 \\
\hline 2 & 6,620 & 10,000 & 4,600 & 5,300 & 6,700 & 7,700 & 23,200 & 12,400 & 22,800 & 96,100 & 39,400 & 19,400 \\
\hline 3 & 6,380 & 9,980 & 3,600 & 5,300 & 6,700 & 7,700 & 22,200 & 11,600 & 22,200 & 101,000 & 38,300 & 18,800 \\
\hline 4 & 6,120 & 9,800 & 3,600 & 5,400 & 6,700 & 7,600 & 20,600 & 11,800 & 22,500 & 112,000 & 32,800 & 18,300 \\
\hline 5 & 5,880 & 9,620 & 3,600 & 5,500 & 6,700 & 7,400 & 19,100 & 12,600 & 38,200 & 122,000 & 30,100 & 18,000 \\
\hline 6 & 6,000 & 9,800 & 3,600 & 5,600 & 6,700 & 6,800 & 17,300 & 13,600 & 52,300 & 113,000 & 28,400 & 18,600 \\
\hline 7 & 6,500 & 9,620 & 3,650 & 5,700 & 6,700 & 5,700 & 15,200 & 13,500 & 48,800 & 105,000 & 27,700 & 23,700 \\
\hline 8 & 6,750 & 9,620 & 3,700 & 5,800 & 6,600 & 5,600 & 13,600 & 13,300 & 44,800 & 108,000 & 27,800 & 32,800 \\
\hline 9 & 7,050 & 9,670 & 3,800 & 5,850 & 6,100 & 6,000 & 12,200 & 13,000 & 41,300 & 114,000 & 28,100 & 22,800 \\
\hline 10 & 7,350 & 9,620 & 3,850 & 5,950 & 5,600 & 9,000 & 11,400 & 12,800 & 37,000 & 123,000 & 28,000 & 22,800 \\
\hline 11 & 7,950 & 9,620 & 3,900 & 6,000 & 5,400 & 11,000 & 10,800 & 12,600 & 34,300 & 102,000 & 27,100 & 23,200 \\
\hline 12 & 8,400 & 9,450 & 3,950 & 6,100 & 5,100 & 12,600 & 11,900 & 12,600 & 34,800 & 78,900 & 24,800 & 21,300 \\
\hline 13 & 8,900 & 9,280 & 3,950 & 6,200 & 4,750 & 13,600 & 13,000 & 12,600 & 48,800 & 65,300 & 25,100 & 21,900 \\
\hline 14 & 9,600 & 9,200 & 4,000 & 6,250 & 4,600 & 13,900 & 14,000 & 12,600 & 53,200 & 56,900 & 24,600 & 22,800 \\
\hline 15 & 9,950 & 9,280 & 4,100 & 6,300 & 4,500 & 14,200 & 13,700 & 14,500 & 52,300 & 52,100 & 25,000 & 27,900 \\
\hline 16 & 10,800 & 9,100 & 4,150 & 6,350 & 4,400 & 14,700 & 13,400 & 16,000 & 51,400 & 50,300 & 24,600 & 29,700 \\
\hline 17 & 14,000 & 9,420 & 4,200 & 6,400 & 4,400 & 17,000 & 13,400 & 16,500 & 48,600 & 52,400 & 25,000 & 26,400 \\
\hline 18 & 15,600 & 9,080 & 4,250 & 6,450 & 4,350 & 34,000 & 13,000 & 16,400 & 46,000 & 54,200 & 24,600 & 24,600 \\
\hline 19 & 15,400 & 6,120 & 4,350 & 6,500 & 4,250 & 52,300 & 13,000 & 15,800 & 44,600 & 51,400 & 22,800 & 22,500 \\
\hline 20 & 14,800 & 4,600 & 4,400 & 6,600 & 4,200 & 83,400 & 12,400 & 15,800 & 40,000 & 47,200 & 22,500 & 21,500 \\
\hline 21 & 14,100 & 3,720 & 4,500 & 6,600 & 4,200 & 130,000 & 12,000 & 15,900 & 43,800 & 44,100 & 22,200 & 20,900 \\
\hline 22 & 13,200 & 4,250 & 4,600 & 6,650 & 4,250 & 162,000 & 11,800 & 15,400 & 53,400 & 42,100 & 22,200 & 20,600 \\
\hline 23 & 12,600 & 3,150 & 4,650 & 6,700 & 4,300 & 85,000 & 11,800 & 15,100 & 52,500 & 40,400 & 22,800 & 20,900 \\
\hline 24 & 12,200 & 3,730 & 4,700 & 6,700 & 4,300 & 54,200 & 11,600 & 15,200 & 62,900 & 39,200 & 22,800 & 20,600 \\
\hline 25 & 11,600 & 4,200 & 4,750 & 6,700 & 4,400 & 48,000 & 11,800 & 15,000 & 77,700 & 38,100 & 22,800 & 20,100 \\
\hline 26 & 11,200 & 4,200 & 4,800 & 6,700 & 4,700 & 46,400 & 12,000 & 14,900 & 70,200 & 35,800 & 22,200 & 19,000 \\
\hline 27 & 10,800 & 4,300 & 4,900 & 6,700 & 5,400 & 43,400 & 11,800 & 14,600 & 67,000 & 32,400 & 21,200 & 19,000 \\
\hline 28 & 10,500 & 4,000 & 4,900 & 6,700 & 6,200 & 40,000 & 11,800 & 21,800 & 81,300 & 30,600 & 21,000 & 18,600 \\
\hline 29 & 10,200 & 4,000 & 5,000 & 6,700 & -.. & 35,300 & 11,400 & 29,100 & 95,900 & 31,600 & 20,200 & 18,800 \\
\hline 30 & 10,000 & 4,300 & 5,100 & 6,700 & -- & 31,900 & 11,600 & 27,000 & 115,000 & 33,000 & 21,000 & 18,600 \\
\hline 31 & 10,200 & --- & 5,100 & 6,700 & $\cdots$ & 26,800 & --. & 24,800 & -- & 34,400 & 20,600 & -.. \\
\hline TOTAL & 307,400 & 222,930 & 133,630 & 192,300 & 148,900 & $1,040,800$ & 426,300 & 480,700 & $1,526,800$ & $2,102,600$ & 802,100 & 653,800 \\
\hline MEAN & 9,916 & 7,431 & 4,311 & 6,203 & 5,318 & 33,570 & 14,210 & 15,510 & 50,890 & 67,830 & 25,870 & 21,790 \\
\hline MAX & 15,600 & 10,200 & 5,380 & 6,700 & 6,700 & 162,000 & 25,300 & 29,100 & 115,000 & 123,000 & 39,400 & 32,800 \\
\hline MIN & 5,880 & 3,150 & 3,600 & 5,200 & 4,200 & 5,600 & 10,800 & 11,600 & 22,200 & 30,600 & 20,200 & 18,000 \\
\hline AC-FT & 609,700 & 442,200 & 265,100 & 381,400 & 295,300 & $2,064,000$ & 845,600 & 953,500 & $3,028,000$ & $4,171,000$ & $1,591,000$ & $1,297,000$ \\
\hline
\end{tabular}


Table 15. Daily discharge for the Missouri River at Pierre (station 06440000) for water years 1934-65—Continued

[--, no data; MAX, maximum value; MIN, minimum value; AC-FT, acre-feet]

\begin{tabular}{|c|c|c|c|c|c|c|c|c|c|c|c|c|}
\hline Day & Oct. & Nov. & Dec. & Jan. & Feb. & Mar. & April & May & June & July & Aug. & Sept. \\
\hline \multicolumn{13}{|c|}{$\begin{array}{l}\text { DISCHARGE, CUBIC FEET PER SECOND, WATER YEAR OCTOBER } 1938 \text { TO SEPTEMBER } 1939 \\
\text { DAILY MEAN VALUES }\end{array}$} \\
\hline 1 & 18,400 & 14,600 & 8,700 & 8,200 & 10,600 & 6,400 & 167,000 & 20,300 & 42,500 & 46,500 & 16,400 & 12,200 \\
\hline 2 & 18,200 & 15,400 & 9,200 & 8,400 & 10,000 & 6,600 & 146,000 & 20,300 & 48,200 & 45,600 & 15,900 & 12,200 \\
\hline 3 & 18,400 & 14,900 & 9,500 & 8,900 & 9,400 & 6,800 & 92,800 & 20,300 & 49,300 & 51,500 & 15,500 & 12,200 \\
\hline 4 & 18,800 & 14,400 & 11,000 & 9,200 & 9,200 & 6,800 & 68,200 & 19,500 & 44,900 & 50,500 & 15,600 & 11,600 \\
\hline 5 & 19,200 & 14,400 & 11,700 & 9,900 & 9,000 & 6,800 & 58,800 & 19,300 & 41,700 & 47,200 & 15,100 & 10,900 \\
\hline 6 & 19,800 & 14,200 & 11,400 & 10,500 & 8,800 & 7,000 & 53,000 & 19,300 & 40,400 & 43,700 & 15,400 & 10,500 \\
\hline 7 & 20,200 & 14,000 & 11,800 & 10,700 & 8,600 & 7,200 & 49,800 & 19,800 & 50,000 & 42,500 & 15,400 & 10,100 \\
\hline 8 & 20,200 & 13,300 & 10,700 & 10,900 & 8,400 & 7,700 & 47,500 & 20,000 & 71,400 & 42,000 & 14,000 & 9,760 \\
\hline 9 & 20,800 & 12,900 & 9,580 & 10,800 & 8,200 & 8,200 & 43,800 & 20,300 & 72,300 & 41,400 & 13,300 & 9,580 \\
\hline 10 & 22,000 & 13,300 & 9,930 & 10,700 & 7,800 & 8,400 & 40,700 & 20,000 & 62,500 & 43,300 & 12,600 & 9,080 \\
\hline 11 & 23,100 & 14,900 & 10,300 & 11,200 & 7,500 & 7,700 & 37,400 & 24,300 & 46,900 & 42,300 & 12,200 & 8,910 \\
\hline 12 & 22,200 & 20,300 & 10,500 & 11,800 & 7,200 & 7,800 & 33,900 & 30,700 & 39,700 & 41,000 & 12,400 & 8,750 \\
\hline 13 & 20,900 & 20,300 & 10,000 & 11,900 & 6,900 & 8,000 & 31,200 & 31,500 & 42,000 & 38,500 & 12,400 & 8,590 \\
\hline 14 & 18,200 & 19,500 & 10,000 & 12,100 & 6,900 & 8,100 & 29,900 & 32,900 & 46,500 & 38,200 & 12,400 & 8,590 \\
\hline 15 & 16,400 & 18,700 & 10,000 & 12,200 & 7,000 & 8,200 & 29,900 & 34,700 & 46,500 & 36,200 & 12,600 & 8,440 \\
\hline 16 & 14,700 & 18,400 & 9,900 & 12,300 & 7,200 & 8,300 & 29,300 & 33,300 & 45,800 & 33,000 & 13,300 & 8,280 \\
\hline 17 & 13,300 & 17,900 & 9,800 & 12,400 & 7,500 & 9,600 & 29,800 & 32,000 & 44,000 & 29,400 & 13,900 & 8,130 \\
\hline 18 & 12,200 & 20,000 & 10,100 & 12,200 & 7,800 & 11,600 & 30,400 & 30,200 & 42,700 & 27,000 & 14,200 & 7,980 \\
\hline 19 & 11,600 & 19,800 & 9,800 & 12,100 & 7,600 & 13,400 & 29,200 & 29,400 & 40,600 & 26,200 & 14,000 & 7,980 \\
\hline 20 & 11,200 & 18,400 & 9,600 & 12,300 & 7,400 & 17,200 & 28,500 & 30,200 & 43,600 & 22,900 & 13,600 & 7,840 \\
\hline 21 & 11,000 & 17,400 & 9,600 & 12,200 & 7,100 & 17,700 & 28,700 & 33,800 & 46,500 & 21,700 & 12,500 & 7,840 \\
\hline 22 & 10,900 & 16,100 & 9,700 & 12,000 & 6,600 & 20,000 & 28,400 & 36,100 & 46,500 & 21,400 & 13,000 & 7,840 \\
\hline 23 & 11,300 & 14,900 & 8,900 & 11,800 & 6,300 & 25,000 & 27,600 & 37,100 & 48,600 & 20,800 & 13,700 & 7,840 \\
\hline 24 & 11,400 & 10,200 & 8,800 & 11,500 & 5,900 & 31,000 & 26,600 & 35,600 & 60,800 & 19,800 & 14,000 & 7,740 \\
\hline 25 & 11,600 & 9,410 & 9,100 & 11,100 & 5,900 & 36,000 & 25,600 & 35,200 & 59,900 & 19,000 & 14,400 & 7,980 \\
\hline 26 & 11,600 & 8,750 & 8,700 & 11,700 & 6,000 & 39,700 & 23,800 & 35,200 & 53,000 & 18,400 & 14,400 & 7,880 \\
\hline 27 & 11,700 & 8,280 & 8,100 & 12,300 & 6,100 & 43,500 & 22,700 & 46,900 & 47,900 & 18,400 & 13,800 & 8,080 \\
\hline 28 & 11,800 & 8,500 & 7,700 & 12,400 & 6,200 & 84,400 & 21,300 & 48,900 & 49,200 & 19,500 & 15,100 & 8,040 \\
\hline 29 & 12,000 & 8,500 & 7,900 & 12,000 & -- & 146,000 & 20,400 & 45,000 & 53,000 & 19,800 & 13,800 & 8,140 \\
\hline 30 & 13,000 & 8,500 & 7,800 & 12,000 & $\ldots$ & 167,000 & 20,500 & 42,000 & 52,200 & 19,000 & 13,000 & 8,040 \\
\hline 31 & 13,900 & --- & 8.000 & 11,400 & -- & 193,000 & --. & 40,300 & -.. & 17,700 & 12,700 & -- \\
\hline TOTAL & 490,000 & 440,140 & 297,810 & 349,100 & 213,100 & 975,100 & $1,322,700$ & 944,400 & $1,479,100$ & $1,004,400$ & 430,600 & 271,030 \\
\hline MEAN & 15,810 & 14,670 & 9,607 & 11,260 & 7,611 & 31,450 & 44,090 & 30,460 & 49,300 & 32,400 & 13,890 & 9,034 \\
\hline MAX & 23,100 & 20,300 & 11,800 & 12,400 & 10,600 & 193,000 & 167,000 & 48,900 & 72,300 & 51,500 & 16,400 & 12,200 \\
\hline MIN & 10,900 & 8,280 & 7,700 & 8,200 & 5,900 & 6,400 & 20,400 & 19,300 & 39,700 & 17,700 & 12,200 & 7,740 \\
\hline AC-FT & 971,900 & 873,000 & 590,700 & 692,400 & 422,700 & $1,934,000$ & $2,624,000$ & $1,873,000$ & $2,934,000$ & $1,992,000$ & 854,100 & 537,600 \\
\hline
\end{tabular}


Table 15. Daily discharge for the Missouri River at Pierre (station 06440000) for water years 1934-65-Continued $[--$, no data; MAX, maximum value; MIN, minimum value; AC-FT, acre-feet $]$

\begin{tabular}{|c|c|c|c|c|c|c|c|c|c|c|c|c|}
\hline Day & Oct. & Nov. & Dec. & Jan. & Feb. & Mar. & April & May & June & July & Aug. & Sept. \\
\hline \multicolumn{13}{|c|}{$\begin{array}{c}\text { DISCHARGE, CUBIC FEET PER SECOND, WATER YEAR OCTOBER } 1939 \text { TO SEPTEMBER } 1940 \\
\text { DAILY MEAN VALUES }\end{array}$} \\
\hline 1 & 7,980 & 8,790 & 10,500 & 3,200 & 4,400 & 5,300 & 11,700 & 37,800 & 19,300 & 30,700 & 19,800 & 18,300 \\
\hline 2 & 8,180 & 8,710 & 10,100 & 3,200 & 4,500 & 5,200 & 13,400 & 31,900 & 18,600 & 29,600 & 22,400 & 17,800 \\
\hline 3 & 8,180 & 8,750 & 10,300 & 3,000 & 4,400 & 5,200 & 15,500 & 26,000 & 18,000 & 27,400 & 21,400 & 17,800 \\
\hline 4 & 8,180 & 8,950 & 10,000 & 2,800 & 4,100 & 5,200 & 17,000 & 25,200 & 18,000 & 25,200 & 20,600 & 17,600 \\
\hline 5 & 8,340 & 9,210 & 9,880 & 2,800 & 3,800 & 5,200 & 15,500 & 26,000 & 17,600 & 23,700 & 20,600 & 16,800 \\
\hline 6 & 8,490 & 9,440 & 9,810 & 2,900 & 3,700 & 5,400 & 13,300 & 24,800 & 17,800 & 22,000 & 22,400 & 18,800 \\
\hline 7 & 8,490 & 9,510 & 9,640 & 3,000 & 3,600 & 5,600 & 14,600 & 22,700 & 20,900 & 20,300 & 26,500 & 18,000 \\
\hline 8 & 8,590 & 9,760 & 9,590 & 3,000 & 3,500 & 5,800 & 15,200 & 21,200 & 23,700 & 19,300 & 27,000 & 18,000 \\
\hline 9 & 8,750 & 9,760 & 9,780 & 2,800 & 3,400 & 6,100 & 15,000 & 20,100 & 25,600 & 18,800 & 26,500 & 18,000 \\
\hline 10 & 8,750 & 9,680 & 9,650 & 2,500 & 3,400 & 6,200 & 13,300 & 19,600 & 26,000 & 17,800 & 24,800 & 17,800 \\
\hline 11 & 8,750 & 9,880 & 9,880 & 2,600 & 3,400 & 6,200 & 12,700 & 19,100 & 29,600 & 16,800 & 22,700 & 17,300 \\
\hline 12 & 8,710 & 9,980 & 9,880 & 2,600 & 3,400 & 6,100 & 12,700 & 18,600 & 39,000 & 16,100 & 21,200 & 17,300 \\
\hline 13 & 8,840 & 10,200 & 9,700 & 2,600 & 3,400 & 5,900 & 12,700 & 18,300 & 55,700 & 15,700 & 20,100 & 15,700 \\
\hline 14 & 8,980 & 10,500 & 9,700 & 2,600 & 3,500 & 5,800 & 12,700 & 17,300 & 42,600 & 16,400 & 19,800 & 14,600 \\
\hline 15 & 8,980 & 10,500 & 9,350 & 2,700 & 3,700 & 5,600 & 15,400 & 16,800 & 37,200 & 19,600 & 19,300 & 13,100 \\
\hline 16 & 9,010 & 10,700 & 9,530 & 2,800 & 4,000 & 5,600 & 34,200 & 16,600 & 38,400 & 21,800 & 19,100 & 11,700 \\
\hline 17 & 8,940 & 10,800 & 9,700 & 2,900 & 4,300 & 5,800 & 34,800 & 16,600 & 40,800 & 21,200 & 19,100 & 10,400 \\
\hline 18 & 8,940 & 11,200 & 9,700 & 3,100 & 4,700 & 7,200 & 28,500 & 17,100 & 36,000 & 19,100 & 19,100 & 9,400 \\
\hline 19 & 8,960 & 11,200 & 9,500 & 3,100 & 5,000 & 7,800 & 25,200 & 17,300 & 29,600 & 17,300 & 18,800 & 8,910 \\
\hline 20 & 9,120 & 11,000 & 9,080 & 3,100 & 5,300 & 8,400 & 20,300 & 16,600 & 27,000 & 16,600 & 18,600 & 8,280 \\
\hline 21 & 9,570 & 11,000 & 8,910 & 3,300 & 5,500 & 8,200 & 16,600 & 15,700 & 24,800 & 16,600 & 18,300 & 7,840 \\
\hline 22 & 9,120 & 11,000 & 9,080 & 3,500 & 5,600 & 7,300 & 14,800 & 15,000 & 23,700 & 17,300 & 18,300 & 7,550 \\
\hline 23 & 9,210 & 11,000 & 8,440 & 3,600 & 5,700 & 6,900 & 16,600 & 16,400 & 25,600 & 17,800 & 18,300 & 7,280 \\
\hline 24 & 9,310 & 11,000 & 7,600 & 3,800 & 5,600 & 6,800 & 19,600 & 21,400 & 33,600 & 17,300 & 18,000 & 7,140 \\
\hline 25 & 9,240 & 11,000 & 5,700 & 4,000 & 5,600 & 6,800 & 20,300 & 24,000 & 34,800 & 17,100 & 18,300 & 7,140 \\
\hline 26 & 9,380 & 11,000 & 5,000 & 4,100 & 5,500 & 6,700 & 19,600 & 23,300 & 36,000 & 17,300 & 18,300 & 7,140 \\
\hline 27 & 9,300 & 11,000 & 4,700 & 4,200 & 5,400 & 6,600 & 18,800 & 22,000 & 33,600 & 17,600 & 18,000 & 7,410 \\
\hline 28 & 9,450 & 10,800 & 4,000 & 4,300 & 5,400 & 6,800 & 18,600 & 21,400 & 30,700 & 18,000 & 17,800 & 7,690 \\
\hline 29 & 9,450 & 10,700 & 3,800 & 4,300 & 5,300 & 7,000 & 23,300 & 21,200 & 29,600 & 18,000 & 17,800 & 7,840 \\
\hline 30 & 9,290 & 10,700 & 3,600 & 4,300 & -.. & 7,300 & 40,800 & 20,600 & 29,600 & 18,300 & 17,800 & 7,980 \\
\hline 31 & 9,390 & --- & 3,400 & 4,400 & -- & 8,400 & --- & 20,100 & --- & 18,300 & 18,000 & --- \\
\hline TOTAL & 275,870 & 307,720 & 259,500 & 101,100 & 129,100 & 198,400 & 562,700 & 650,700 & 883,400 & 609,000 & 628,700 & 380,600 \\
\hline MEAN & 8,899 & 10,260 & 8,371 & 3,261 & 4,452 & 6,400 & 18,760 & 20,990 & 29,450 & 19,650 & 20,280 & 12,690 \\
\hline MAX & 9,570 & 11,200 & 10,500 & 4,400 & 5,700 & 8,400 & 40,800 & 37,800 & 55,700 & 30,700 & 27,000 & 18,800 \\
\hline MIN & 7,980 & 8,710 & 3,400 & 2,500 & 3,400 & 5,200 & 11,700 & 15,000 & 17,600 & 15,700 & 17,800 & 7,140 \\
\hline AC-FT & 547,200 & 610,400 & 514,700 & 200,500 & 256,100 & 393,500 & $1,116,000$ & $1,291,000$ & $1,752,000$ & $1,208,000$ & $1,247,000$ & 754,900 \\
\hline
\end{tabular}


Table 15. Daily discharge for the Missouri River at Pierre (station 06440000) for water years 1934-65-Continued [---, no data; MAX, maximum value; MIN, minimum value; AC-FT, acre-feet]

\begin{tabular}{|c|c|c|c|c|c|c|c|c|c|c|c|c|}
\hline Day & Oct. & Nov. & Dec. & Jan. & Feb. & Mar. & April & May & June & July & Aug. & Sept. \\
\hline \multicolumn{13}{|c|}{$\begin{array}{c}\text { DISCHARGE, CUBIC FEET PER SECOND, WATER YEAR OCTOBER } 1940 \text { TO SEPTEMBER } 1941 \\
\text { DAILY MEAN VALUES }\end{array}$} \\
\hline 1 & 8,900 & 10,700 & 4,600 & 5,300 & 5,800 & 6,300 & 27,600 & 18,600 & 23,600 & 32,300 & 19,500 & 19,200 \\
\hline 2 & 9,540 & 10,700 & 4,300 & 5,200 & 5,900 & 6,300 & 22,500 & 18,600 & 23,200 & 30,400 & 19,200 & 19,500 \\
\hline 3 & 9,700 & 10,900 & 4,200 & 5,000 & 6,000 & 6,300 & 20,800 & 18,600 & 30,400 & 28,600 & 19,500 & 20,100 \\
\hline 4 & 9,540 & 11,000 & 4,600 & 5,000 & 6,200 & 6,300 & 20,800 & 19,200 & 39,100 & 27,600 & 19,500 & 19,800 \\
\hline 5 & 9,380 & 11,200 & 5,300 & 5,200 & 6,100 & 6,300 & 19,200 & 19,200 & 40,800 & 29,000 & 20,100 & 19,200 \\
\hline 6 & 10,000 & 11,200 & 6,300 & 5,400 & 5,900 & 6,200 & 21,100 & 18,900 & 50,900 & 29,400 & 20,800 & 19,500 \\
\hline 7 & 10,400 & 11,000 & 7,200 & 5,700 & 5,800 & 6,200 & 23,200 & 18,900 & 46,300 & 27,200 & 23,600 & 20,100 \\
\hline 8 & 10,500 & 11,000 & 7,800 & 5,900 & 5,600 & 6,200 & 38,000 & 18,600 & 46,300 & 25,100 & 27,600 & 20,400 \\
\hline 9 & 10,900 & 10,900 & 8,100 & 6,200 & 5,400 & 6,200 & 36,900 & 19,500 & 60,400 & 23,600 & 26,800 & 22,500 \\
\hline 10 & 10,900 & 10,000 & 8,300 & 6,500 & 5,400 & 6,200 & 30,800 & 20,100 & 74,500 & 22,800 & 23,600 & 24,000 \\
\hline 11 & 17,000 & 9,700 & 8,300 & 6,800 & 5,300 & 6,300 & 29,900 & 20,100 & 101,000 & 22,200 & 21,800 & 25,500 \\
\hline 12 & 26,400 & 8,150 & 8,200 & 6,900 & 5,300 & 6,500 & 32,300 & 20,100 & 125,000 & 21,800 & 21,100 & 31,300 \\
\hline 13 & 25,100 & 3,520 & 8,000 & 6,900 & 5,400 & 7,000 & 33,300 & 20,100 & 127,000 & 21,400 & 21,100 & 45,000 \\
\hline 14 & 21,400 & 2,300 & 7,800 & 6,800 & 5,600 & 7,500 & 47,000 & 19,800 & 106,000 & 21,100 & 21,400 & 45,700 \\
\hline 15 & 19,500 & 2,300 & 7,800 & 6,700 & 5,700 & 8,000 & 39,100 & 19,800 & 82,800 & 21,100 & 21,400 & 46,300 \\
\hline 16 & 17,500 & 2,300 & 7,700 & 6,300 & 5,900 & 8,700 & 29,900 & 19,800 & 65,900 & 22,500 & 21,400 & 34,800 \\
\hline 17 & 15,500 & 2,400 & 7,700 & 6,000 & 6,100 & 9,300 & 28,100 & 20,400 & 58,000 & 25,100 & 24,000 & 26,800 \\
\hline 18 & 14,200 & 2,500 & 7,800 & 5,700 & 6,200 & 9,700 & 24,000 & 21,100 & 55,800 & 23,200 & 26,800 & 21,100 \\
\hline 19 & 13,200 & 2,600 & 7,900 & 5,300 & 6,300 & 9,900 & 22,500 & 21,100 & 48,900 & 21,100 & 26,400 & 18,900 \\
\hline 20 & 12,600 & 2,800 & 8,200 & 5,000 & 6,300 & 10,100 & 21,400 & 20,100 & 40,200 & 19,800 & 24,000 & 18,100 \\
\hline 21 & 12,300 & 3,100 & 8,600 & 4,800 & 6,400 & 10,200 & 20,100 & 18,900 & 36,400 & 18,600 & 22,200 & 17,800 \\
\hline 22 & 11,900 & 3,400 & 9,100 & 4,700 & 6,400 & 10,200 & 19,500 & 18,300 & 34,800 & 18,300 & 21,100 & 17,500 \\
\hline 23 & 11,700 & 3,800 & 9,600 & 4,400 & 6,400 & 10,300 & 19,500 & 18,100 & 35,300 & 18,300 & 20,800 & 17,500 \\
\hline 24 & 11,700 & 4,200 & 9,700 & 4,300 & 6,400 & 10,500 & 18,900 & 24,700 & 39,100 & 18,600 & 20,400 & 18,300 \\
\hline 25 & 11,500 & 4,400 & 9,700 & 4,400 & 6,400 & 10,700 & 18,600 & 29,400 & 43,200 & 20,100 & 21,400 & 17,800 \\
\hline 26 & 11,700 & 4,700 & 9,200 & 4,500 & 6,400 & 11,100 & 19,500 & 28,100 & 39,700 & 20,400 & 21,400 & 16,800 \\
\hline 27 & 11,700 & 4,800 & 8,000 & 4,700 & 6,300 & 12,300 & 20,100 & 26,400 & 36,400 & 20,400 & 19,500 & 16,000 \\
\hline 28 & 11,500 & 4,800 & 7,200 & 5,000 & 6,300 & 12,400 & 20,400 & 24,700 & 35,300 & 20,400 & 18,100 & 15,800 \\
\hline 29 & 11,200 & 4,900 & 6,600 & 5,200 & --- & 12,300 & 19,800 & 24,000 & 34,300 & 19,800 & 17,500 & 15,100 \\
\hline 30 & 10,900 & 4,800 & 6,200 & 5,400 & --- & 12,600 & 18,900 & 23,600 & 33,300 & 19,500 & 17,800 & 14,900 \\
\hline 31 & 10,700 & --- & 5,700 & 5,700 & $\cdots$ & 17,300 & -. & 23,200 & -- & 19,800 & 18,900 & -.- \\
\hline TOTAL & 408,960 & 190,070 & 229,700 & 170,900 & 167,200 & 275,400 & 763,700 & 652,000 & $1,613,900$ & 709,500 & 668,700 & 685,300 \\
\hline MEAN & 13,190 & 6,336 & 7,410 & 5,513 & 5,971 & 8,884 & 25,460 & 21,030 & 53,800 & 22,890 & 21,570 & 22,840 \\
\hline MAX & 26,400 & 11,200 & 9,700 & 6,900 & 6,400 & 17,300 & 47,000 & 29,400 & 127,000 & 32,300 & 27,600 & 46,300 \\
\hline MIN & 8,900 & 2,300 & 4,200 & 4,300 & 5,300 & 6,200 & 18,600 & 18,100 & 23,200 & 18,300 & 17,500 & 14,900 \\
\hline AC-FT & 811,200 & 377,000 & 455,600 & 339,000 & 331,600 & 546,300 & $1,515,000$ & $1,293,000$ & $3,201,000$ & $1,407,000$ & $1,326,000$ & $1,359,000$ \\
\hline
\end{tabular}


Table 15. Daily discharge for the Missouri River at Pierre (station 06440000) for water years 1934-65-Continued [---, no data; MAX, maximum value; MIN, minimum value; AC-FT, acre-feet]

\begin{tabular}{|c|c|c|c|c|c|c|c|c|c|c|c|c|}
\hline Day & Oct. & Nov. & Dec. & Jan. & Feb. & Mar. & April & May & June & July & Aug. & Sept. \\
\hline \multicolumn{13}{|c|}{$\begin{array}{l}\text { DISCHARGE, CUBIC FEET PER SECOND, WATER YEAR OCTOBER } 1941 \text { TO SEPTEMBER } 1942 \\
\text { DAILY MEAN VALUES }\end{array}$} \\
\hline 1 & 14,900 & 15,100 & 7,120 & 7,800 & 5,900 & 7,000 & 8,300 & 50,200 & 47,600 & 38,600 & 25,100 & 18,300 \\
\hline 2 & 15,100 & 14,600 & 6,840 & 7,600 & 6,300 & 7,100 & 13,800 & 57,300 & 48,900 & 38,600 & 22,200 & 18,900 \\
\hline 3 & 15,100 & 14,400 & 6,980 & 7,800 & 6,700 & 7,200 & 33,800 & 48,200 & 55,100 & 39,100 & 21,100 & 19,200 \\
\hline 4 & 15,500 & 14,200 & 7,700 & 7,900 & 7,100 & 7,300 & 25,500 & 40,800 & 71,800 & 40,800 & 20,800 & 18,900 \\
\hline 5 & 15,800 & 14,200 & 8,450 & 8,000 & 7,500 & 7,400 & 32,800 & 36,900 & 75,400 & 42,600 & 20,100 & 18,600 \\
\hline 6 & 16,500 & 14,400 & 8,300 & 8,000 & 8,000 & 7,500 & 46,300 & 33,800 & 82,800 & 42,600 & 19,800 & 18,900 \\
\hline 7 & 18,100 & 14,200 & 8,150 & 7,500 & 8,600 & 7,600 & 62,700 & 30,800 & 83,800 & 39,100 & 20,800 & 18,900 \\
\hline 8 & 20,800 & 14,200 & 8,200 & 7,000 & 9,200 & 7,700 & 47,600 & 30,800 & 71,000 & 35,800 & 21,800 & 19,200 \\
\hline 9 & 21,400 & 13,600 & 8,500 & 6,700 & 9,300 & 7,800 & 31,800 & 30,400 & 63,500 & 33,800 & 23,200 & 19,500 \\
\hline 10 & 20,800 & 13,400 & 7,000 & 6,300 & 9,200 & 7,900 & 25,100 & 27,200 & 71,800 & 32,800 & 24,000 & 19,500 \\
\hline 11 & 20,100 & 13,200 & 6,500 & 6,000 & 8,900 & 8,000 & 21,800 & 26,800 & 90,800 & 33,300 & 23,200 & 19,500 \\
\hline 12 & 19,500 & 13,000 & 4,300 & 5,800 & 8,600 & 8,100 & 19,500 & 28,600 & 86,800 & 34,300 & 22,800 & 19,500 \\
\hline 13 & 22,800 & 12,800 & 4,200 & 5,400 & 8,500 & 8,200 & 17,800 & 39,100 & 77,200 & 35,800 & 22,200 & 19,200 \\
\hline 14 & 27,200 & 13,000 & 4,000 & 5,000 & 8,600 & 8,300 & 17,300 & 40,200 & 68,400 & 36,900 & 21,100 & 18,900 \\
\hline 15 & 26,800 & 13,000 & 4,000 & 4,800 & 8,700 & 8,400 & 16,800 & 42,600 & 68,400 & 37,400 & 20,400 & 18,600 \\
\hline 16 & 24,300 & 13,000 & 4,200 & 4,600 & 8,800 & 8,500 & 17,000 & 38,000 & 71,000 & 38,000 & 20,100 & 18,300 \\
\hline 17 & 21,400 & 13,000 & 4,500 & 4,500 & 8,800 & 8,000 & 17,500 & 59,600 & 76,300 & 38,000 & 19,800 & 18,300 \\
\hline 18 & 19,800 & 13,000 & 5,000 & 4,400 & 8,800 & 8,150 & 17,800 & 64,300 & 75,400 & 38,600 & 19,500 & 18,300 \\
\hline 19 & 18,900 & 12,800 & 5,500 & 4,400 & 8,900 & 8,600 & 17,500 & 81,900 & 68,400 & 37,400 & 19,200 & 18,100 \\
\hline 20 & 17,800 & 12,400 & 6,000 & 4,400 & 9,000 & 10,200 & 17,800 & 68,400 & 60,400 & 34,800 & 18,900 & 18,100 \\
\hline 21 & 16,800 & 12,300 & 6,700 & 4,400 & 8,500 & 18,600 & 18,100 & 55,800 & 52,300 & 31,800 & 18,900 & 18,300 \\
\hline 22 & 16,300 & 11,900 & 7,500 & 4,400 & 8,100 & 55,800 & 17,800 & 45,000 & 45,700 & 28,600 & 18,900 & 18,600 \\
\hline 23 & 15,800 & 11,700 & 8,100 & 4,400 & 7,800 & 48,900 & 17,800 & 36,900 & 44,400 & 27,600 & 18,600 & 19,200 \\
\hline 24 & 15,300 & 11,200 & 8,200 & 4,400 & 7,500 & 58,000 & 18,300 & 33,800 & 45,000 & 25,900 & 18,300 & 19,800 \\
\hline 25 & 15,300 & 10,500 & 8,200 & 4,400 & 7,300 & 53,000 & 18,900 & 31,800 & 46,300 & 24,300 & 17,800 & 20,100 \\
\hline 26 & 16,000 & 9,860 & 8,300 & 4,500 & 7,300 & 48,900 & 19,500 & 30,400 & 38,600 & 22,800 & 17,500 & 19,800 \\
\hline 27 & 16,000 & 8,300 & 8,300 & 4,600 & 7,300 & 37,400 & 21,100 & 29,000 & 39,100 & 22,800 & 17,500 & 20,400 \\
\hline 28 & 15,500 & 7,550 & 8,400 & 4,800 & 7,200 & 25,500 & 25,100 & 26,800 & 41,400 & 25,900 & 17,500 & 20,800 \\
\hline 29 & 16,000 & 7,400 & 8,300 & 5,000 & ... & 15,800 & 22,800 & 27,600 & 39,100 & 25,500 & 17,500 & 21,100 \\
\hline 30 & 16,000 & 7,550 & 8,200 & 5,200 & -- & 10,900 & 25,900 & 27,200 & 38,000 & 25,100 & 18,100 & 21,800 \\
\hline 31 & 15,500 &.- & 8,000 & 5,500 & --- & 8,000 & --- & 28,600 & --- & 26,800 & 18,300 & - \\
\hline TOTAL & 567,100 & 369,760 & 213,640 & 175,500 & 226,400 & 539,750 & 713,800 & $1,248,800$ & $1,844,700$ & $1,035,400$ & 625,000 & 576,600 \\
\hline MEAN & 18,290 & 12,330 & 6,892 & 5,661 & 8,086 & 17,410 & 23,790 & 40,280 & 61,490 & 33,400 & 20,160 & 19,220 \\
\hline MAX & 27,200 & 15,100 & 8,500 & 8,000 & 9,300 & 58,000 & 62,700 & 81,900 & 90,800 & 42,600 & 25,100 & 21,800 \\
\hline MIN & 14,900 & 7,400 & 4,000 & 4,400 & 5,900 & 7,000 & 8,300 & 26,800 & 38,000 & 22,800 & 17,500 & 18,100 \\
\hline AC-FT & $1,125,000$ & 733,400 & 423,800 & 348,100 & 449,100 & $1,071,000$ & $1,416,000$ & $2,477,000$ & $3,659,000$ & $2,054,000$ & $1,240,000$ & $1,144,000$ \\
\hline
\end{tabular}


Table 15. Daily discharge for the Missouri River at Pierre (station 06440000) for water years 1934-65—Continued

[---, no data; MAX, maximum value; MIN, minimum value; AC-FT, acre-feet]

\begin{tabular}{|c|c|c|c|c|c|c|c|c|c|c|c|c|}
\hline Day & Oct. & Nov. & Dec. & Jan. & Feb. & Mar. & April & May & June & July & Aug. & Sept. \\
\hline \multicolumn{13}{|c|}{$\begin{array}{l}\text { DISCHARGE, CUBIC FEET PER SECOND, WATER YEAR OCTOBER } 1942 \text { TO SEPTEMBER } 1943 \\
\text { DAILY MEAN VALUES }\end{array}$} \\
\hline 1 & 22,200 & 19,800 & 7,400 & 7,600 & 6,400 & 30,000 & 197,000 & 25,600 & 22,100 & 87,200 & 30,000 & 32,200 \\
\hline 2 & 22,200 & 19,500 & 7,260 & 8,400 & 6,400 & 25,000 & 202,000 & 25,900 & 24,400 & 85,600 & 28,900 & 34,200 \\
\hline 3 & 22,200 & 19,500 & 6,400 & 9,000 & 6,200 & 24,000 & 211,000 & 26,900 & 24,400 & 84,000 & 28,600 & 35,000 \\
\hline 4 & 21,800 & 19,800 & 6,000 & 9,400 & 6,000 & 23,000 & 245,000 & 26,900 & 24,400 & 84,000 & 28,600 & 35,000 \\
\hline 5 & 21,100 & 20,100 & 5,600 & 9,600 & 5,800 & 25,000 & 263,000 & 26,600 & 45,600 & 89,600 & 28,200 & 33,000 \\
\hline 6 & 21,400 & 20,800 & 5,300 & 9,600 & 5,600 & 26,000 & 278,000 & 26,900 & 71,000 & 80,900 & 27,500 & 30,700 \\
\hline 7 & 21,100 & 21,100 & 5,100 & 9,400 & 5,400 & 25,000 & 239,000 & 26,900 & 80,900 & 78,600 & 26,800 & 29,300 \\
\hline 8 & 21,100 & 21,800 & 5,000 & 9,200 & 5,300 & 25,000 & 122,000 & 25,800 & 85,600 & 81,700 & 27,200 & 27,800 \\
\hline 9 & 20,400 & 22,200 & 4,900 & 8,800 & 5,200 & 25,000 & 63,400 & 24,100 & 81,700 & 83,200 & 25,400 & 27,200 \\
\hline 10 & 20,100 & 22,500 & 4,800 & 8,400 & 5,200 & 25,000 & 52,800 & 23,100 & 74,700 & 78,600 & 25,100 & 27,500 \\
\hline 11 & 19,800 & 22,200 & 4,700 & 8,000 & 5,100 & 30,000 & 44,700 & 22,800 & 66,000 & 71,700 & 24,400 & 28,600 \\
\hline 12 & 19,500 & 21,800 & 4,600 & 7,500 & 5,200 & 36,000 & 40,000 & 22,500 & 63,300 & 67,400 & 24,800 & 28,900 \\
\hline 13 & 18,900 & 21,400 & 4,700 & 7,200 & 5,300 & 32,000 & 37,900 & 22,800 & 51,800 & 66,700 & 25,100 & 28,900 \\
\hline 14 & 18,900 & 21,800 & 4,800 & 7,000 & 5,600 & 29,000 & 37,200 & 23,400 & 80,100 & 68,100 & 24,800 & 28,600 \\
\hline 15 & 18,900 & 21,800 & 4,800 & 6,800 & 6,200 & 27,000 & 35,800 & 24,400 & 77,800 & 64,600 & 24,400 & 28,200 \\
\hline 16 & 18,600 & 22,200 & 4,800 & 6,600 & 7,000 & 25,000 & 34,500 & 25,400 & 80,100 & 63,300 & 23,400 & 28,200 \\
\hline 17 & 18,300 & 21,800 & 4,800 & 6,400 & 7,300 & 20,000 & 34,100 & 25,800 & 68,100 & 62,000 & 22,100 & 27,500 \\
\hline 18 & 18,600 & 20,400 & 4,800 & 6,200 & 7,600 & 21,000 & 33,100 & 25,400 & 61,300 & 59,400 & 21,500 & 27,500 \\
\hline 19 & 18,300 & 18,600 & 4,900 & 6,100 & 7,800 & 20,500 & 31,500 & 24,400 & 71,700 & 56,100 & 21,500 & 27,500 \\
\hline 20 & 18,600 & 17,300 & 5,000 & 6,000 & 8,500 & 19,500 & 31,100 & 23,800 & 74,700 & 52,400 & 21,800 & 27,200 \\
\hline 21 & 18,600 & 16,000 & 5,000 & 5,900 & 9,500 & 19,000 & 31,100 & 23,400 & 74,000 & 47,800 & 22,100 & 27,200 \\
\hline 22 & 18,900 & 15,100 & 5,000 & 5,800 & 10,500 & 18,500 & 32,100 & 23,400 & 68,100 & 44,000 & 22,500 & 27,200 \\
\hline 23 & 18,900 & 14,400 & 4,900 & 5,800 & 11,500 & 18,500 & 33,100 & 23,100 & 58,000 & 41,000 & 24,100 & 26,800 \\
\hline 24 & 19,200 & 13,800 & 4,900 & 5,800 & 13,000 & 19,000 & 29,800 & 23,400 & 57,400 & 39,200 & 28,200 & 26,800 \\
\hline 25 & 19,200 & 13,600 & 4,800 & 5,900 & 17,000 & 19,500 & 27,500 & 22,100 & 60,000 & 37,900 & 30,400 & 26,800 \\
\hline 26 & 19,500 & 13,000 & 4,700 & 6,100 & 25,000 & 30,000 & 26,200 & 21,200 & 66,700 & 37,100 & 30,400 & 27,200 \\
\hline 27 & 19,800 & 12,300 & 4,700 & 6,300 & 30,000 & 35,000 & 24,000 & 20,800 & 77,800 & 35,000 & 30,400 & 27,200 \\
\hline 28 & 20,400 & 11,700 & 4,700 & 6,400 & 35,000 & 50,000 & 24,600 & 19,900 & 84,000 & 33,400 & 31,100 & 27,500 \\
\hline 29 & 20,400 & 10,900 & 5,000 & 6,400 & -- & 100,000 & 24,000 & 20,500 & 91,200 & 31,900 & 31,100 & 27,800 \\
\hline 30 & 20,400 & 8,600 & 5,800 & 6,400 & --- & 150,000 & 24,000 & 22,100 & 90,400 & 31,100 & 31,100 & 27,500 \\
\hline 31 & 20,100 & --- & 6,800 & 6,400 & --- & 179,000 & -- & 21,800 & --- & 30,700 & 31,100 & --- \\
\hline TOTAL & 617,400 & 545,800 & 161,960 & 224,400 & 274,600 & $1,151,500$ & $2,509,500$ & 741,100 & $1,957,300$ & $1,874,200$ & 822,600 & 865,000 \\
\hline MEAN & 19,920 & 18,190 & 5,225 & 7,239 & 9,807 & 37,150 & 83,650 & 23,910 & 65,240 & 60,460 & 26,540 & 28,830 \\
\hline MAX & 22,200 & 22,500 & 7,400 & 9,600 & 35,000 & 179,000 & 278,000 & 26,900 & 91,200 & 89,600 & 31,100 & 35,000 \\
\hline MIN & 18,300 & 8,600 & 4,600 & 5,800 & 5,100 & 18,500 & 24,000 & 19,900 & 22,100 & 30,700 & 21,500 & 26,800 \\
\hline AC-FT & $1,225,000$ & $1,083,000$ & 321,200 & 445,100 & 544,700 & $2,284,000$ & $4,978,000$ & $1,470,000$ & $3,882,000$ & $3,717,000$ & $1,632,000$ & $1,716,000$ \\
\hline
\end{tabular}


Table 15. Daily discharge for the Missouri River at Pierre (station 06440000) for water years 1934-65 - Continued [---, no data; MAX, maximum value; MIN, minimum value; AC-FT, acre-feet]

\begin{tabular}{|c|c|c|c|c|c|c|c|c|c|c|c|c|}
\hline Day & Oct. & Nov. & Dec. & Jan. & Feb. & Mar. & April & May & June & July & Aug. & Sept. \\
\hline \multicolumn{13}{|c|}{$\begin{array}{l}\text { DISCHARGE, CUBIC FEET PER SECOND, WATER YEAR OCTOBER } 1943 \text { TO SEPTEMBER } 1944 \\
\text { DAILY MEAN VALUES }\end{array}$} \\
\hline 1 & 27,200 & 26,800 & 23,800 & 11,000 & 15,400 & 9,200 & 22,700 & 16,800 & 33,800 & 104,000 & 22,800 & 26,100 \\
\hline 2 & 26,500 & 26,800 & 23,400 & 11,700 & 16,300 & 9,100 & 26,000 & 16,200 & 33,100 & 92,000 & 22,800 & 26,100 \\
\hline 3 & 26,100 & 26,800 & 24,100 & 12,800 & 16,800 & 9,100 & 40,000 & 16,000 & 32,100 & 90,100 & 23,100 & 25,800 \\
\hline 4 & 26,100 & 27,200 & 23,400 & 13,800 & 16,500 & 9,000 & 102,000 & 15,200 & 33,100 & 92,000 & 23,800 & 25,400 \\
\hline 5 & 26,500 & 27,500 & 24,100 & 14,800 & 16,200 & 8,900 & 102,000 & 14,400 & 32,800 & 93,000 & 25,400 & 25,100 \\
\hline 6 & 26,800 & 28,200 & 24,100 & 15,600 & 16,000 & 8,800 & 121,000 & 14,400 & 42,400 & 82,700 & 25,400 & 24,400 \\
\hline 7 & 27,500 & 28,900 & 23,400 & 16,300 & 15,900 & 8,800 & 175,000 & 13,200 & 62,000 & 69,200 & 25,100 & 24,100 \\
\hline 8 & 28,200 & 28,900 & 22,800 & 16,700 & 15,700 & 8,800 & 179,000 & 12,700 & 67,000 & 62,000 & 24,400 & 24,100 \\
\hline 9 & 28,200 & 28,900 & 22,100 & 17,200 & 15,800 & 8,800 & 186,000 & 13,200 & 67,700 & 60,600 & 25,400 & 23,400 \\
\hline 10 & 28,600 & 28,600 & 21,800 & 17,700 & 16,000 & 8,700 & 166,000 & 13,400 & 71,600 & 62,000 & 27,200 & 22,500 \\
\hline 11 & 28,200 & 28,200 & 21,500 & 17,900 & 16,400 & 8,700 & 149,000 & 13,700 & 74,800 & 59,900 & 28,600 & 21,800 \\
\hline 12 & 27,800 & 27,500 & 20,800 & 17,900 & 16,500 & 8,700 & 149,000 & 14,700 & 78,200 & 59,900 & 30,000 & 21,200 \\
\hline 13 & 28,200 & 26,800 & 19,000 & 17,800 & 16,300 & 8,700 & 113,000 & 14,700 & 74,000 & 56,000 & 30,700 & 20,800 \\
\hline 14 & 27,800 & 26,100 & 17,000 & 17,400 & 15,800 & 8,800 & 73,200 & 16,200 & 71,600 & 56,600 & 30,400 & 20,800 \\
\hline 15 & 27,800 & 26,100 & 15,000 & 16,900 & 15,000 & 8,800 & 60,600 & 17,000 & 67,700 & 52,800 & 29,300 & 21,500 \\
\hline 16 & 28,200 & 26,100 & 14,000 & 16,000 & 14,300 & 8,900 & 53,400 & 16,000 & 68,400 & 50,400 & 28,200 & 21,500 \\
\hline 17 & 27,800 & 25,800 & 13,000 & 15,000 & 13,500 & 9,000 & 46,600 & 15,200 & 80,000 & 48,800 & 27,200 & 20,500 \\
\hline 18 & 27,800 & 25,400 & 13,000 & 14,000 & 12,800 & 9,200 & 40,400 & 14,400 & 84,500 & 47,100 & 25,800 & 19,000 \\
\hline 19 & 27,200 & 24,800 & 12,500 & 12,700 & 12,000 & 9,300 & 33,800 & 13,400 & 101,000 & 45,600 & 26,100 & 18,600 \\
\hline 20 & 25,800 & 23,800 & 12,500 & 11,300 & 11,400 & 9,400 & 29,500 & 12,700 & 114,000 & 42,800 & 26,100 & 18,600 \\
\hline 21 & 25,100 & 23,100 & 12,000 & 10,500 & 11,000 & 9,600 & 26,900 & 13,000 & 127,000 & 39,600 & 26,500 & 18,600 \\
\hline 22 & 25,100 & 22,800 & 12,000 & 9,600 & 10,400 & 9,800 & 25,200 & 13,400 & 125,000 & 37,200 & 26,500 & 19,000 \\
\hline 23 & 25,100 & 22,800 & 11,700 & 9,000 & 10,100 & 10,000 & 23,300 & 15,500 & 124,000 & 34,800 & 25,800 & 19,000 \\
\hline 24 & 25,800 & 23,100 & 11,400 & 8,900 & 9,900 & 11,000 & 22,700 & 17,600 & 131,000 & 33,100 & 25,800 & 19,000 \\
\hline 25 & 26,100 & 23,100 & 10,800 & 9,000 & 9,700 & 13,500 & 21,400 & 36,800 & 136,000 & 31,500 & 26,500 & 19,300 \\
\hline 26 & 26,800 & 23,400 & 10,500 & 9,200 & 9,500 & 14,000 & 20,800 & 53,400 & 127,000 & 29,500 & 25,800 & 19,300 \\
\hline 27 & 27,500 & 23,400 & 10,300 & 9,800 & 9,400 & 15,500 & 20,000 & 55,300 & 115,000 & 28,200 & 26,100 & 19,300 \\
\hline 28 & 27,500 & 23,400 & 10,000 & 10,700 & 9,300 & 17,000 & 18,900 & 50,400 & 127,000 & 26,200 & 26,100 & 19,000 \\
\hline 29 & 27,500 & 23,100 & 10,100 & 12,000 & 9,200 & 18,100 & 17,800 & 41,600 & 134,000 & 25,200 & 26,500 & 18,600 \\
\hline 30 & 27,200 & 23,800 & 10,200 & 13,000 & -. & 19,200 & 17,300 & 37,200 & 122,000 & 24,600 & 26,500 & 18,600 \\
\hline 31 & 27,200 & --- & 10,400 & 14,300 & --- & 21,000 & -- & 35,200 & -.- & 24,000 & 26,500 & -. \\
\hline TOTAL & 839,200 & 771,200 & 510,700 & 420,500 & 393,100 & 337,400 & $2,082,500$ & 662,900 & $2,557,800$ & $1,661,400$ & 816,400 & 641,000 \\
\hline MEAN & 27,070 & 25,710 & 16,470 & 13,560 & 13,560 & 10,880 & 69,420 & 21,380 & 85,260 & 53,590 & 26,340 & 21,370 \\
\hline MAX & 28,600 & 28,900 & 24,100 & 17,900 & 16,800 & 21,000 & 186,000 & 55,300 & 136,000 & 104,000 & 30,700 & 26,100 \\
\hline MIN & 25,100 & 22,800 & 10,000 & 8,900 & 9,200 & 8,700 & 17,300 & 12,700 & 32,100 & 24,000 & 22,800 & 18,600 \\
\hline AC-FT & $1,665,000$ & $1,530,000$ & $1,013,000$ & 834,100 & 779,700 & 669,200 & $4,131,000$ & $1,315,000$ & $5,073,000$ & $3,295,000$ & $1,619,000$ & $1,271,000$ \\
\hline
\end{tabular}


Table 15. Daily discharge for the Missouri River at Pierre (station 06440000) for water years 1934-65—Continued

[---, no data; MAX, maximum value; MIN, minimum value; AC-FT, acre-feet]

\begin{tabular}{|c|c|c|c|c|c|c|c|c|c|c|c|c|}
\hline Day & Oct. & Nov. & Dec. & Jan. & Feb. & Mar. & April & May & June & July & Aug. & Sept. \\
\hline \multicolumn{13}{|c|}{$\begin{array}{l}\text { DISCHARGE, CUBIC FEET PER SECOND, WATER YEAR OCTOBER } 1944 \text { TO SEPTEMBER } 1945 \\
\text { DAILY MEAN VALUES }\end{array}$} \\
\hline 1 & 19,000 & 25,400 & 18,300 & 12,500 & 20,800 & 13,100 & 39,600 & 11,200 & 18,600 & 76,200 & 32,200 & 18,300 \\
\hline 2 & 19,600 & 23,800 & 15,800 & 12,500 & 20,500 & 13,700 & 36,600 & 11,200 & 19,600 & 76,200 & 31,100 & 18,600 \\
\hline 3 & 19,600 & 22,500 & 14,900 & 11,600 & 19,900 & 13,400 & 33,000 & 11,200 & 20,500 & 74,000 & 28,900 & 19,300 \\
\hline 4 & 20,200 & 21,800 & 13,700 & 12,500 & 19,600 & 13,100 & 30,400 & 11,200 & 20,500 & 71,700 & 27,500 & 19,600 \\
\hline 5 & 20,500 & 21,500 & 13,400 & 12,200 & 19,600 & 12,200 & 28,900 & 10,900 & 23,100 & 67,400 & 26,500 & 19,300 \\
\hline 6 & 21,200 & 21,200 & 12,500 & 12,200 & 19,000 & 10,600 & 26,100 & 10,900 & 25,400 & 54,900 & 25,800 & 18,600 \\
\hline 7 & 21,800 & 21,200 & 12,500 & 11,600 & 19,300 & 9,400 & 23,400 & 11,600 & 33,800 & 44,000 & 25,400 & 18,300 \\
\hline 8 & 22,500 & 21,500 & 14,300 & 10,900 & 18,300 & 9,400 & 21,200 & 12,500 & 47,200 & 37,900 & 23,100 & 18,600 \\
\hline 9 & 22,100 & 21,200 & 14,000 & 10,600 & 18,300 & 10,300 & 20,500 & 13,100 & 48,900 & 36,200 & 21,500 & 19,000 \\
\hline 10 & 21,800 & 21,200 & 11,900 & 9,700 & 17,700 & 12,500 & 20,200 & 13,400 & 51,800 & 34,600 & 20,200 & 20,200 \\
\hline 11 & 21,200 & 21,200 & 11,200 & 9,700 & 16,800 & 19,300 & 19,300 & 13,400 & 58,700 & 33,400 & 19,300 & 21,800 \\
\hline 12 & 20,500 & 21,800 & 11,600 & 10,300 & 15,800 & 23,100 & 18,000 & 12,800 & 60,000 & 33,400 & 19,300 & 22,500 \\
\hline 13 & 19,600 & 22,800 & 12,500 & 11,200 & 15,500 & 29,300 & 17,400 & 12,500 & 60,600 & 34,600 & 19,600 & 22,500 \\
\hline 14 & 19,600 & 24,100 & 14,900 & 12,200 & 15,200 & 40,100 & 17,100 & 11,900 & 63,300 & 36,600 & 20,800 & 22,800 \\
\hline 15 & 19,300 & 25,100 & 14,600 & 11,900 & 16,800 & 51,800 & 16,800 & 10,900 & 56,800 & 37,100 & 21,200 & 23,100 \\
\hline 16 & 19,000 & 25,400 & 15,200 & 12,500 & 19,300 & 47,200 & 16,800 & 10,600 & 49,500 & 37,500 & 24,100 & 24,100 \\
\hline 17 & 19,000 & 25,800 & 15,500 & 12,500 & 19,300 & 66,000 & 16,200 & 10,300 & 44,000 & 36,600 & 23,100 & 25,800 \\
\hline 18 & 19,000 & 25,800 & 14,900 & 12,800 & 17,700 & 80,100 & 15,500 & 10,300 & 42,000 & 36,200 & 22,800 & 27,200 \\
\hline 19 & 19,900 & 25,400 & 15,500 & 14,000 & 17,400 & 86,400 & 15,200 & 10,000 & 41,500 & 37,500 & 22,800 & 27,500 \\
\hline 20 & 20,500 & 25,400 & 16,500 & 15,200 & 14,900 & 98,600 & 14,300 & 10,600 & 40,500 & 37,100 & 22,100 & 26,800 \\
\hline 21 & 21,500 & 24,800 & 17,400 & 16,500 & 14,900 & 84,800 & 13,700 & 11,900 & 39,600 & 37,500 & 22,100 & 27,200 \\
\hline 22 & 24,800 & 25,400 & 17,400 & 17,400 & 14,900 & 74,700 & 13,700 & 13,100 & 41,000 & 37,900 & 22,100 & 27,200 \\
\hline 23 & 28,900 & 25,400 & 18,000 & 18,000 & 14,600 & 73,200 & 13,100 & 14,600 & 41,000 & 39,200 & 22,500 & 27,500 \\
\hline 24 & 30,000 & 24,800 & 17,400 & 19,000 & 15,200 & 74,000 & 12,800 & 15,500 & 38,300 & 41,000 & 21,500 & 27,800 \\
\hline 25 & 29,600 & 24,800 & 15,800 & 19,600 & 16,200 & 79,400 & 12,500 & 17,400 & 35,800 & 41,000 & 21,200 & 28,600 \\
\hline 26 & 29,300 & 22,800 & 15,800 & 19,300 & 15,200 & 78,600 & 12,200 & 18,600 & 33,400 & 40,100 & 20,800 & 29,600 \\
\hline 27 & 28,600 & 21,800 & 16,200 & 19,600 & 14,600 & 73,200 & 12,200 & 19,900 & 31,100 & 38,800 & 20,200 & 30,400 \\
\hline 28 & 28,200 & 21,500 & 17,100 & 21,200 & 14,000 & 58,000 & 11,900 & 19,900 & 28,900 & 37,500 & 19,900 & 30,400 \\
\hline 29 & 27,800 & 20,800 & 14,900 & 22,800 & --- & 54,900 & 11,600 & 20,200 & 33,400 & 36,200 & 19,000 & 30,000 \\
\hline 30 & 27,200 & 19,900 & 14,600 & 22,500 & --. & 55,500 & 11,600 & 19,300 & 66,000 & 34,600 & 18,600 & 28,900 \\
\hline 31 & 26,500 & -.. & 14,000 & 21,800 & --- & 45,100 & -. & 19,000 & --- & 33,400 & 18,300 & -- \\
\hline TOTAL & 708,300 & 696,100 & 462,300 & 456,300 & 481,300 & $1,411,000$ & 571,800 & 419,900 & $1,214,800$ & $1,350,300$ & 703,500 & 721,500 \\
\hline MEAN & 22,850 & 23,200 & 14,910 & 14,720 & 17,190 & 45,520 & 19,060 & 13,550 & 40,490 & 43,560 & 22,690 & 24,050 \\
\hline MAX & 30,000 & 25,800 & 18,300 & 22,800 & 20,800 & 98,600 & 39,600 & 20,200 & 66,000 & 76,200 & 32,200 & 30,400 \\
\hline MIN & 19,000 & 19,900 & 11,200 & 9,700 & 14,000 & 9,400 & 11,600 & 10,000 & 18,600 & 33,400 & 18,300 & 18,300 \\
\hline AC-FT & $1,405,000$ & $1,381,000$ & 917,000 & 905,100 & 954,700 & $2,799,000$ & $1,134,000$ & 832,900 & $2,410,000$ & $2,678,000$ & $1,395,000$ & $1,431,000$ \\
\hline
\end{tabular}


Table 15. Daily discharge for the Missouri River at Pierre (station 06440000) for water years 1934-65—Continued

[---, no data; MAX, maximum value; MIN, minimum value; AC-FT, acre-feet]

\begin{tabular}{|c|c|c|c|c|c|c|c|c|c|c|c|c|}
\hline Day & Oct. & Nov. & Dec. & Jan. & Feb. & Mar. & April & May & June & July & Aug. & Sept. \\
\hline \multicolumn{13}{|c|}{$\begin{array}{c}\text { DISCHARGE, CUBIC FEET PER SECOND, WATER YEAR OCTOBER } 1945 \text { TO SEPTEMBER } 1946 \\
\text { DAILY MEAN VALUES }\end{array}$} \\
\hline 1 & 28,200 & 27,800 & 14,600 & 4,600 & 8,700 & 8,100 & 24,300 & 13,200 & 24,300 & 45,900 & 20,500 & 24,300 \\
\hline 2 & 28,500 & 26,500 & 9,900 & 4,600 & 8,300 & 8,500 & 18,600 & 14,600 & 34,100 & 44,600 & 19,100 & 24,000 \\
\hline 3 & 29,300 & 25,800 & 10,300 & 4,700 & 8,300 & 9,500 & 17,100 & 16,800 & 32,000 & 49,100 & 18,600 & 24,000 \\
\hline 4 & 28,500 & 24,600 & 9,100 & 4,600 & 8,500 & 9,500 & 16,100 & 32,000 & 28,900 & 51,100 & 18,100 & 24,300 \\
\hline 5 & 28,200 & 23,700 & 7,900 & 4,700 & 8,300 & 9,700 & 16,600 & 26,100 & 32,400 & 44,600 & 17,800 & 24,000 \\
\hline 6 & 27,100 & 23,100 & 7,200 & 4,400 & 8,300 & 9,700 & 17,100 & 23,400 & 37,200 & 39,600 & 17,300 & 24,000 \\
\hline 7 & 26,100 & 22,200 & 7,000 & 4,400 & 8,100 & 10,600 & 17,600 & 21,900 & 36,300 & 36,700 & 16,300 & 24,300 \\
\hline 8 & 25,200 & 21,900 & 6,600 & 4,900 & 8,100 & 12,100 & 16,800 & 19,700 & 34,500 & 35,400 & 15,600 & 24,300 \\
\hline 9 & 25,200 & 21,100 & 5,500 & 5,300 & 8,100 & 12,800 & 16,600 & 18,100 & 32,000 & 35,400 & 14,900 & 24,000 \\
\hline 10 & 25,200 & 20,500 & 5,800 & 5,800 & 7,900 & 13,700 & 15,800 & 18,100 & 29,600 & 34,500 & 14,200 & 23,700 \\
\hline 11 & 25,500 & 20,000 & 6,800 & 6,100 & 7,500 & 15,100 & 15,400 & 19,700 & 27,800 & 34,500 & 13,700 & 24,300 \\
\hline 12 & 26,100 & 17,800 & 7,300 & 6,400 & 7,300 & 16,100 & 15,100 & 21,600 & 26,800 & 33,600 & 12,800 & 24,900 \\
\hline 13 & 26,100 & 15,400 & 7,900 & 6,600 & 7,200 & 21,900 & 15,100 & 21,900 & 26,100 & 34,100 & 12,100 & 25,200 \\
\hline 14 & 26,100 & 12,800 & 8,500 & 7,000 & 6,800 & 24,600 & 14,900 & 21,900 & 30,400 & 36,300 & 12,100 & 25,800 \\
\hline 15 & 26,500 & 11,700 & 7,700 & 8,100 & 6,400 & 29,300 & 14,600 & 20,500 & 46,500 & 37,700 & 13,200 & 25,500 \\
\hline 16 & 26,100 & 11,200 & 6,000 & 8,700 & 6,600 & 23,100 & 14,400 & 19,400 & 49,800 & 38,600 & 16,600 & 28,200 \\
\hline 17 & 25,800 & 11,200 & 6,800 & 9,500 & 6,600 & 22,500 & 13,900 & 18,600 & 49,800 & 39,100 & 20,200 & 36,700 \\
\hline 18 & 25,800 & 11,200 & 8,300 & 10,100 & 6,800 & 21,900 & 13,200 & 18,400 & 56,300 & 41,200 & 21,900 & 37,200 \\
\hline 19 & 25,200 & 11,000 & 9,300 & 11,200 & 7,000 & 21,400 & 13,200 & 18,600 & 69,200 & 42,900 & 22,500 & 33,600 \\
\hline 20 & 25,500 & 10,600 & 9,100 & 11,900 & 7,200 & 26,800 & 12,800 & 19,100 & 79,900 & 37,700 & 22,200 & 28,900 \\
\hline 21 & 25,200 & 10,100 & 8,100 & 11,900 & 7,300 & 34,900 & 12,300 & 19,400 & 72,000 & 33,200 & 22,200 & 26,800 \\
\hline 22 & 24,900 & 9,500 & 7,300 & 11,700 & 7,500 & 37,700 & 11,900 & 19,100 & 63,000 & 30,400 & 22,500 & 26,100 \\
\hline 23 & 24,600 & 9,700 & 6,800 & 11,200 & 7,700 & 31,200 & 11,700 & 19,400 & 54,000 & 29,300 & 23,700 & 25,200 \\
\hline 24 & 24,900 & 9,500 & 6,300 & 10,800 & 7,700 & 26,500 & 11,700 & 20,000 & 51,100 & 29,600 & 24,900 & 25,500 \\
\hline 25 & 25,500 & 9,900 & 6,300 & 10,800 & 7,700 & 26,500 & 11,400 & 27,500 & 54,800 & 32,000 & 25,500 & 26,500 \\
\hline 26 & 27,200 & 11,200 & 6,100 & 10,600 & 8,100 & 27,100 & 11,200 & 32,000 & 54,000 & 33,600 & 24,900 & 27,500 \\
\hline 27 & 28,200 & 12,800 & 6,100 & 10,300 & 8,100 & 26,500 & 11,000 & 24,900 & 52,600 & 31,600 & 24,600 & 28,200 \\
\hline 28 & 29,300 & 15,800 & 5,800 & 9,300 & 7,900 & 25,200 & 10,600 & 22,200 & 49,800 & 28,200 & 25,200 & 28,200 \\
\hline 29 & 30,000 & 17,100 & 5,200 & 8,900 & -. & 34,100 & 11,000 & 21,600 & 48,400 & 25,500 & 25,200 & 27,500 \\
\hline 30 & 30,000 & 14,900 & 4,900 & 8,900 & ... & 46,500 & 12,100 & 21,900 & 49,100 & 23,700 & 24,900 & 26,500 \\
\hline 31 & 29,300 & -.- & 4,700 & 8,900 & --. & 35,800 & --- & 23,400 & -.. & 21,900 & 24,900 & -- \\
\hline TOTAL & 829,300 & 490,600 & 229,200 & 246,900 & 214,000 & 678,900 & 434,100 & 655,000 & $1,332,700$ & $1,111,600$ & 608,200 & 799,200 \\
\hline MEAN & 26,750 & 16,350 & 7,394 & 7,965 & 7,643 & 21,900 & 14,470 & 21,130 & 44,420 & 35,860 & 19,620 & 26,640 \\
\hline MAX & 30,000 & 27,800 & 14,600 & 11,900 & 8,700 & 46,500 & 24,300 & 32,000 & 79,900 & 51,100 & 25,500 & 37,200 \\
\hline MIN & 24,600 & 9,500 & 4,700 & 4,400 & 6,400 & 8,100 & 10,600 & 13,200 & 24,300 & 21,900 & 12,100 & 23,700 \\
\hline AC-FT & $1,645,000$ & 973,100 & 454,600 & 489,700 & 424,500 & $1,347,000$ & 861,000 & $1,299,000$ & $2,643,000$ & $2,205,000$ & $1,206,000$ & $1,585,000$ \\
\hline
\end{tabular}


Table 15. Daily discharge for the Missouri River at Pierre (station 06440000) for water years 1934-65-Continued [--, no data; MAX, maximum value; MIN, minimum value; AC-FT, acre-feet]

\begin{tabular}{|c|c|c|c|c|c|c|c|c|c|c|c|c|}
\hline Day & Oct. & Nov. & Dec. & Jan. & Feb. & Mar. & April & May & June & July & Aug. & Sept. \\
\hline \multicolumn{13}{|c|}{$\begin{array}{l}\text { DISCHARGE, CUBIC FEET PER SECOND, WATER YEAR OCTOBER } 1946 \text { TO SEPTEMBER } 1947 \\
\text { DAILY MEAN VALUES }\end{array}$} \\
\hline 1 & 26,400 & 20,500 & 4,180 & 7,200 & 9,700 & 15,000 & 230,000 & 33,200 & 38,200 & 84,000 & 38,200 & 29,300 \\
\hline 2 & 25,400 & 20,800 & 3,910 & 7,800 & 9,700 & 15,000 & 138,000 & 35,000 & 36,800 & 84,000 & 38,200 & 28,900 \\
\hline 3 & 24,400 & 20,800 & 5,400 & 8,400 & 9,700 & 15,300 & 89,700 & 34,500 & 35,000 & 74,400 & 38,200 & 28,900 \\
\hline 4 & 24,100 & 20,500 & 4,580 & 8,800 & 9,100 & 15,700 & 63,900 & 35,400 & 34,500 & 72,700 & 37,700 & 28,900 \\
\hline 5 & 24,100 & 21,100 & 5,400 & 9,400 & 9,100 & 16,400 & 60,200 & 35,000 & 40,200 & 71,900 & 35,900 & 28,200 \\
\hline 6 & 23,800 & 21,400 & 5,530 & 9,800 & 8,800 & 16,800 & 59,400 & 33,200 & 47,700 & 66,300 & 34,500 & 27,500 \\
\hline 7 & 24,100 & 21,600 & 6,100 & 10,100 & 9,400 & 17,100 & 62,400 & 32,800 & 48,300 & 58,700 & 33,200 & 27,100 \\
\hline 8 & 24,400 & 20,500 & 6,500 & 10,600 & 9,800 & 17,100 & 54,400 & 31,600 & 41,300 & 51,600 & 32,800 & 27,100 \\
\hline 9 & 28,200 & 18,800 & 6,600 & 10,700 & 10,600 & 16,400 & 47,700 & 32,800 & 40,200 & 47,100 & 34,500 & 28,600 \\
\hline 10 & 28,600 & 17,800 & 6,700 & 10,900 & 10,900 & 14,600 & 44,100 & 34,100 & 46,500 & 45,200 & 36,800 & 30,400 \\
\hline 11 & 28,900 & 16,800 & 6,700 & 10,900 & 11,300 & 13,800 & 40,700 & 34,500 & 63,200 & 45,900 & 38,200 & 31,600 \\
\hline 12 & 37,700 & 16,400 & 6,500 & 10,700 & 11,200 & 12,300 & 40,700 & 36,300 & 63,200 & 47,700 & 38,700 & 32,000 \\
\hline 13 & 40,700 & 15,700 & 6,500 & 10,300 & 10,900 & 10,700 & 46,500 & 52,300 & 51,600 & 53,000 & 42,400 & 32,400 \\
\hline 14 & 35,900 & 15,000 & 6,600 & 9,700 & 10,400 & 9,400 & 57,900 & 53,700 & 47,700 & 57,200 & 42,400 & 32,000 \\
\hline 15 & 32,000 & 14,600 & 6,900 & 8,800 & 10,300 & 9,100 & 55,100 & 54,400 & 46,500 & 57,200 & 40,200 & 32,400 \\
\hline 16 & 30,400 & 14,400 & 7,400 & 8,700 & 11,200 & 9,200 & 55,800 & 60,200 & 47,700 & 55,100 & 39,700 & 32,400 \\
\hline 17 & 29,300 & 14,200 & 8,100 & 8,500 & 12,200 & 9,400 & 56,500 & 64,700 & 55,800 & 53,000 & 39,200 & 32,400 \\
\hline 18 & 28,200 & 13,600 & 9,000 & 8,100 & 13,400 & 9,500 & 57,900 & 64,700 & 57,900 & 52,300 & 37,700 & 32,000 \\
\hline 19 & 26,400 & 13,200 & 10,000 & 7,800 & 12,900 & 9,800 & 55,100 & 62,400 & 56,500 & 53,700 & 37,200 & 31,600 \\
\hline 20 & 24,800 & 12,700 & 12,000 & 7,400 & 13,200 & 10,100 & 48,300 & 55,100 & 53,000 & 53,000 & 37,700 & 31,200 \\
\hline 21 & 24,100 & 12,300 & 12,900 & 7,300 & 14,200 & 12,700 & 43,500 & 50,300 & 54,400 & 50,900 & 38,700 & 31,600 \\
\hline 22 & 24,400 & 10,100 & 12,900 & 7,200 & 15,300 & 17,800 & 41,300 & 45,900 & 67,800 & 47,100 & 37,700 & 32,800 \\
\hline 23 & 25,100 & 7,560 & 13,200 & 7,300 & 15,500 & 19,100 & 37,200 & 44,100 & 96,800 & 44,100 & 36,300 & 33,700 \\
\hline 24 & 24,800 & 6,340 & 12,700 & 7,700 & 15,000 & 22,200 & 36,300 & 42,400 & 137,000 & 41,800 & 35,400 & 34,100 \\
\hline 25 & 24,100 & 4,990 & 12,300 & 8,400 & 14,200 & 27,100 & 36,800 & 42,400 & 156,000 & 39,700 & 34,500 & 34,500 \\
\hline 26 & 23,500 & 5,120 & 11,000 & 8,500 & 13,800 & 27,500 & 36,300 & 44,700 & 141,000 & 39,200 & 33,200 & 34,100 \\
\hline 27 & 22,800 & 4,180 & 10,300 & 8,700 & 14,400 & 35,000 & 35,400 & 45,200 & 131,000 & 39,200 & 32,400 & 34,100 \\
\hline 28 & 22,200 & 4,180 & 9,200 & 8,800 & 14,400 & 86,800 & 34,100 & 43,500 & 121,000 & 38,700 & 31,600 & 33,700 \\
\hline 29 & 21,900 & 4,320 & 5,500 & 8,700 & --- & 51,600 & 33,700 & 40,200 & 107,000 & 38,200 & 30,800 & 34,500 \\
\hline 30 & 21,100 & 4,580 & 3,900 & 8,800 & --- & 45,200 & 33,700 & 38,700 & 92,600 & 37,700 & 30,400 & 34,500 \\
\hline 31 & 20,800 & --- & 6,900 & 9,200 & --- & 159,000 & --- & 38,700 & -.. & 38,200 & 29,700 & --. \\
\hline TOTAL & 822,600 & 414,070 & 245,400 & 275,200 & 330,600 & 766,700 & $1,732,600$ & $1,352,000$ & $2,056,400$ & $1,638,800$ & $1,124,100$ & 942,500 \\
\hline MEAN & 26,540 & 13,800 & 7,916 & 8,877 & 11,810 & 24,730 & 57,750 & 43,610 & 68,550 & 52,860 & 36,260 & 31,420 \\
\hline MAX & 40,700 & 21,600 & 13,200 & 10,900 & 15,500 & 159,000 & 230,000 & 64,700 & 156,000 & 84,000 & 42,400 & 34,500 \\
\hline MIN & 20,800 & 4,180 & 3,900 & 7,200 & 8,800 & 9,100 & 33,700 & 31,600 & 34,500 & 37,700 & 29,700 & 27,100 \\
\hline AC-FT & $1,632,000$ & 821,300 & 486,800 & 545,900 & 655,700 & $1,521,000$ & $3,437,000$ & $2,682,000$ & $4,079,000$ & $3,251,000$ & $2,230,000$ & $1,869,000$ \\
\hline
\end{tabular}


Table 15. Daily discharge for the Missouri River at Pierre (station 06440000) for water years 1934-65—Continued

[---, no data; MAX, maximum value; MIN, minimum value; AC-FT, acre-feet $]$

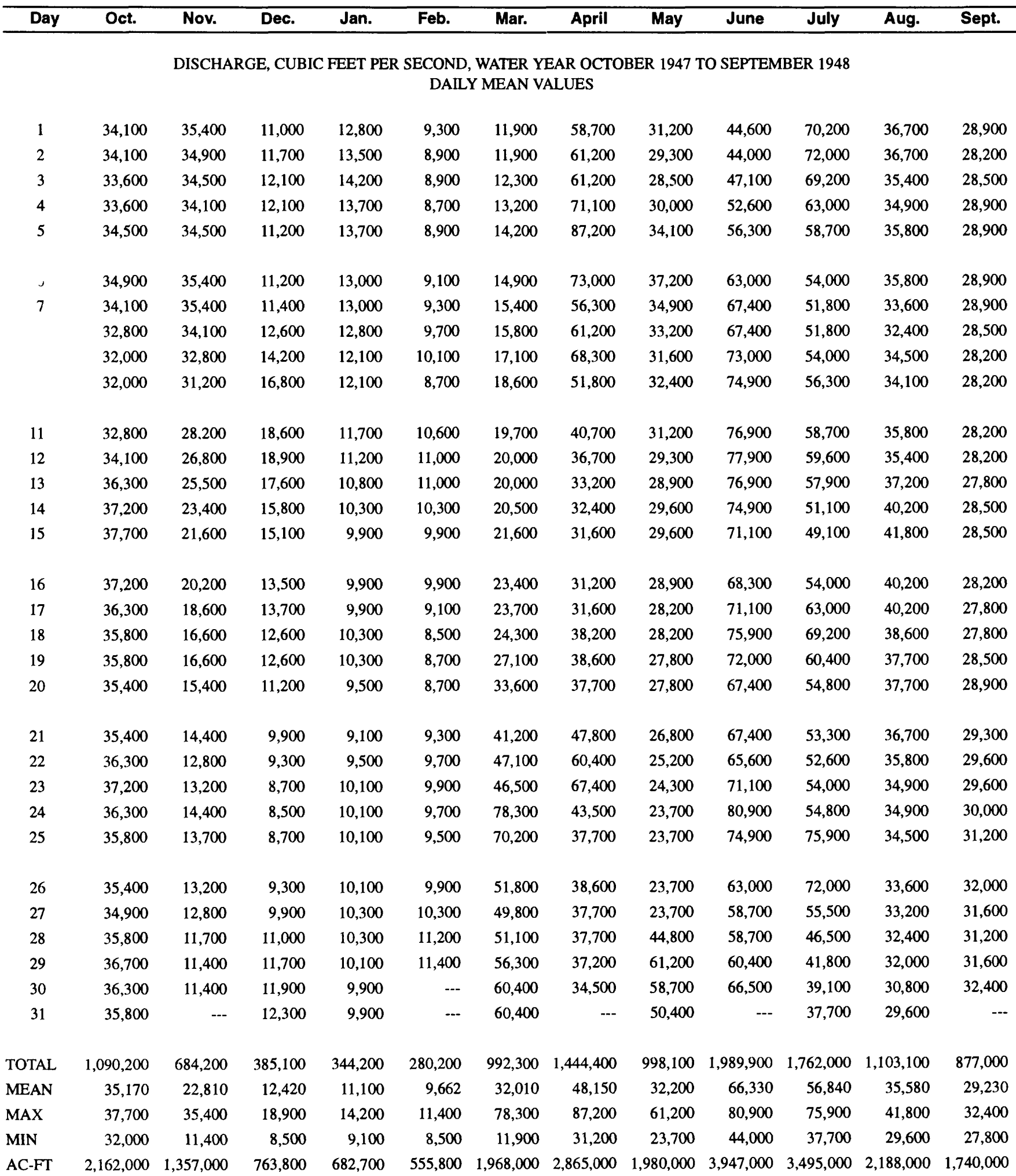


Table 15. Daily discharge for the Missouri River at Pierre (station 06440000) for water years 1934-65-Continued [---, no data; MAX, maximum value; MIN, mininum value; AC-FT, acre-feet]

\begin{tabular}{|c|c|c|c|c|c|c|c|c|c|c|c|c|}
\hline Day & Oct. & Nov. & Dec. & Jan. & Feb. & Mar. & April & May & June & July & Aug. & Sept. \\
\hline \multicolumn{13}{|c|}{$\begin{array}{c}\text { DISCHARGE, CUBIC FEET PER SECOND, WATER YEAR OCTOBER } 1948 \text { TO SEPTEMBER } 1949 \\
\text { DÄILY MEAN VALUES }\end{array}$} \\
\hline 1 & 36,300 & 36,300 & 17,800 & 9,500 & 12,300 & 12,600 & 64,700 & 28,900 & 34,500 & 36,700 & 27,500 & 25,800 \\
\hline 2 & 40,200 & 37,200 & 17,800 & 10,800 & 12,300 & 12,600 & 83,000 & 28,600 & 36,300 & 36,300 & 27,800 & 25,500 \\
\hline 3 & 39,100 & 37,200 & 17,800 & 11,400 & 12,500 & 12,300 & 121,000 & 28,600 & 32,800 & 35,400 & 26,800 & 25,500 \\
\hline 4 & 37,200 & 35,400 & 17,600 & 11,400 & 12,300 & 12,300 & 142,000 & 29,300 & 31,200 & 34,100 & 25,800 & 25,500 \\
\hline 5 & 36,300 & 34,500 & 16,000 & 11,400 & 12,300 & 14,600 & 180,000 & 29,700 & 30,000 & 33,200 & 25,200 & 25,500 \\
\hline 6 & 36,300 & 34,900 & 14,000 & 11,700 & 12,600 & 18,900 & 171,000 & 28,200 & 29,600 & 32,400 & 24,900 & 25,800 \\
\hline 7 & 38,200 & 35,400 & 12,000 & 11,900 & 13,000 & 23,100 & 170,000 & 27,100 & 35,400 & 32,400 & 24,300 & 25,800 \\
\hline 8 & 40,200 & 35,800 & 10,500 & 11,400 & 13,000 & 28,200 & 167,000 & 26,800 & 41,200 & 31,600 & 24,300 & 25,500 \\
\hline 9 & 39,600 & 35,400 & 10,000 & 11,000 & 13,200 & 30,400 & 108,000 & 27,100 & 40,200 & 30,000 & 25,200 & 25,500 \\
\hline 10 & 37,700 & 34,100 & 9,000 & 11,400 & 13,500 & 31,600 & 77,800 & 27,800 & 38,200 & 28,900 & 25,800 & 25,800 \\
\hline 11 & 36,300 & 32,400 & 7,500 & 11,200 & 12,800 & 32,000 & 64,700 & 27,800 & 36,300 & 28,500 & 25,800 & 25,800 \\
\hline 12 & 35,800 & 30,800 & 6,500 & 11,900 & 12,800 & 33,200 & 55,800 & 27,800 & 35,400 & 27,800 & 25,500 & 25,800 \\
\hline 13 & 35,400 & 29,300 & 5,500 & 12,600 & 12,600 & 34,100 & 47,700 & 27,100 & 35,400 & 27,800 & 25,200 & 25,800 \\
\hline 14 & 35,400 & 27,500 & 4,500 & 13,000 & 12,800 & 31,200 & 39,700 & 26,500 & 32,800 & 27,500 & 25,500 & 25,800 \\
\hline 15 & 35,400 & 26,100 & 3,500 & 12,300 & 12,600 & 26,800 & 35,000 & 26,100 & 31,600 & 28,500 & 26,100 & 25,800 \\
\hline 16 & 35,400 & 24,900 & 3,500 & 12,300 & 12,300 & 24,000 & 31,600 & 26,100 & 33,200 & 30,000 & 26,800 & 26,100 \\
\hline 17 & 36,300 & 23,700 & 3,000 & 12,600 & 12,800 & 22,200 & 29,700 & 25,200 & 44,600 & 30,400 & 27,500 & 26,500 \\
\hline 18 & 36,300 & 22,500 & 3,000 & 12,100 & 12,300 & 20,500 & 28,900 & 24,000 & 47,100 & 30,400 & 28,200 & 26,800 \\
\hline 19 & 36,300 & 21,400 & 4,300 & 12.200 & 12,100 & 19,700 & 27,800 & 24,300 & 51,100 & 29,300 & 27,500 & 27,100 \\
\hline 20 & 36,700 & 20,000 & 4,600 & 12,200 & 12,100 & 19,400 & 27,100 & 24,900 & 57,100 & 28,200 & 27,100 & 27,800 \\
\hline 21 & 37,700 & 19,100 & 5,200 & 12,700 & 11,900 & 27,000 & 26,400 & 25,500 & 54,000 & 27,500 & 26,800 & 27,800 \\
\hline 22 & 37,700 & 18,400 & 5,300 & 12,700 & 11,700 & 37,000 & 27,500 & 26,800 & 50,400 & 27,500 & 26,100 & 27,500 \\
\hline 23 & 36,300 & 18,600 & 5,500 & 12,700 & 12,100 & 48,000 & 30,400 & 28,200 & 46,500 & 27,100 & 25,200 & 27,100 \\
\hline 24 & 34,900 & 18,600 & 5,500 & 11,800 & 12,300 & 60,000 & 32,400 & 41,200 & 42,900 & 27,100 & 25,200 & 26,100 \\
\hline 25 & 34,100 & 18,400 & 6,000 & 12,000 & 12,600 & 56,300 & 31,200 & 42,900 & 40,700 & 27,500 & 25,500 & 25,500 \\
\hline 26 & 33,600 & 18,600 & 6,500 & 12,000 & 12,600 & 65,600 & 29,700 & 43,500 & 39,600 & 26,800 & 25,500 & 25,200 \\
\hline 27 & 33,600 & 18,600 & 7,000 & 12,100 & 12,800 & 71,100 & 28,200 & 40,700 & 38,200 & 25,800 & 26,100 & 24,900 \\
\hline 28 & 33,600 & 18,400 & 7,500 & 11,900 & 12,100 & 67,800 & 27,500 & 36,700 & 37,200 & 24,900 & 26,800 & 24,900 \\
\hline 29 & 34,900 & 18,100 & 8,000 & 13,000 & --- & 67,100 & 27,800 & 33,600 & 36,300 & 25,200 & 27,100 & 24,600 \\
\hline 30 & 34,900 & 18,100 & 8,300 & 12,600 & --- & 78,600 & 28,900 & 32,000 & 36,700 & 26,800 & 26,800 & 24,300 \\
\hline 31 & 35,400 & -.. & 8,900 & 12,600 & --- & 67,100 & -- & 32,800 & --- & 27,500 & 26,100 & -- \\
\hline TOTAL & $1,127,100$ & 799,700 & 262,100 & 370,400 & 350,300 & $1,105,300$ & $1,962,500$ & 925,800 & $1,176,500$ & 913,100 & 810,000 & 777,400 \\
\hline MEAN & 36,360 & 26,660 & 8,455 & 11,950 & 12,510 & 35,650 & 65,420 & 29,860 & 39,220 & 29,450 & 26,130 & 25,910 \\
\hline MAX & 40,200 & 37,200 & 17,800 & 13,000 & 13,500 & 78,600 & 180,000 & 43,500 & 57,100 & 36,700 & 28,200 & 27,800 \\
\hline MIN & 33,600 & 18,100 & 3,000 & 9,500 & 11,700 & 12,300 & 26,400 & 24,000 & 29,600 & 24,900 & 24,300 & 24,300 \\
\hline AC-FT & $2,236,000$ & $1,586,000$ & 519,900 & 734,700 & 694,800 & $2,192,000$ & $3,893,000$ & $1,836,000$ & $2,334,000$ & $1,811,000$ & $1,607,000$ & $1,542,000$ \\
\hline
\end{tabular}


Table 15. Daily discharge for the Missouri River at Pierre (station 06440000) for water years 1934-65—Continued $[-\cdots$, no data; MAX, maximum value; MIN, minimum value; AC-FT, acre-feet $]$

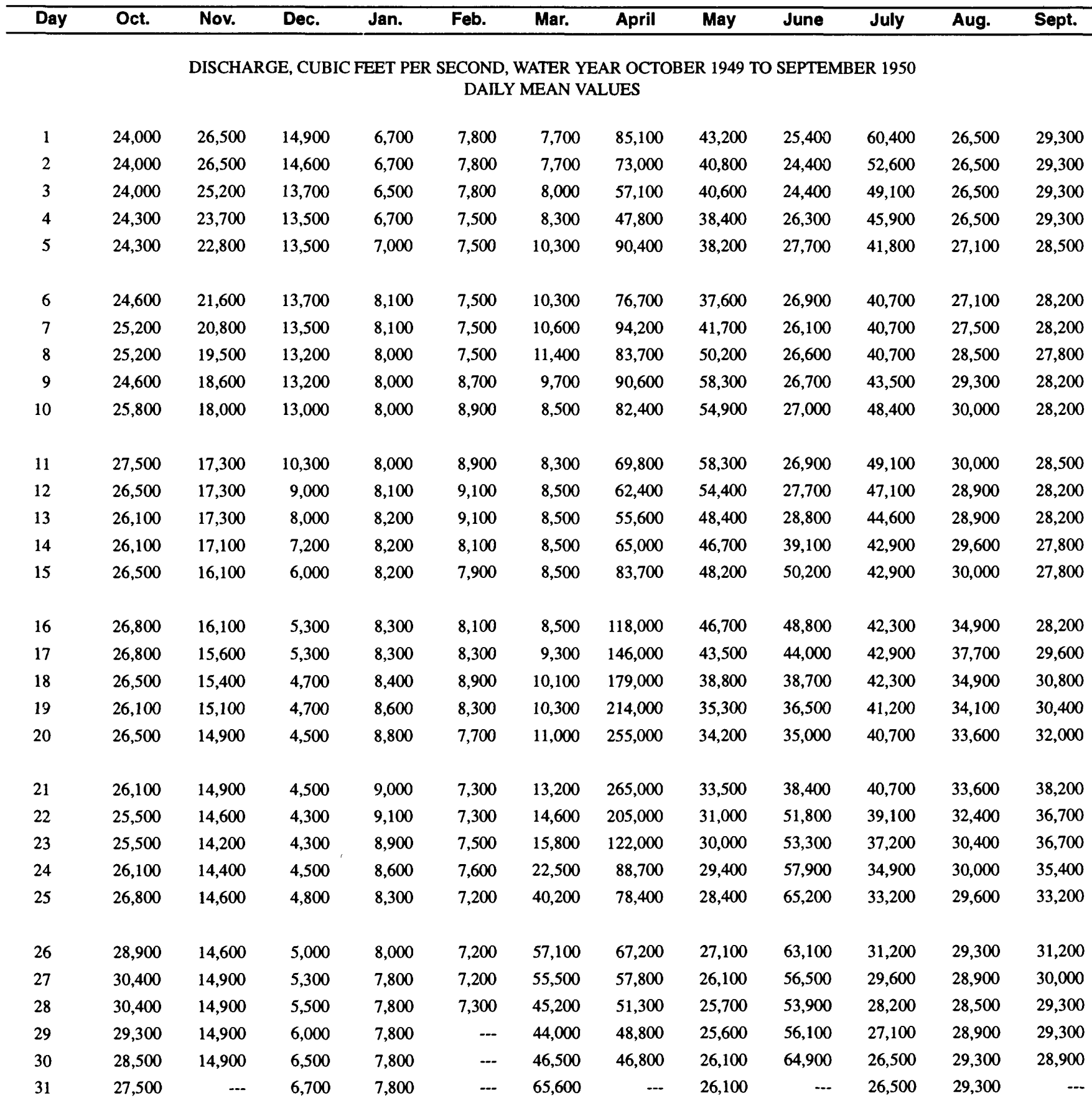

\begin{tabular}{|c|c|c|c|c|c|c|c|c|c|c|c|c|}
\hline TOTAL & 816,400 & 532,300 & 259,200 & 247,800 & 221,500 & 604,200 & $3,060,500$ & $1,207,400$ & $1,198,300$ & $1,254,000$ & 928,300 & 906,700 \\
\hline MEAN & 26,340 & 17,740 & 8,361 & 7,994 & 7,911 & 19,490 & 102,000 & 38,950 & 39,940 & 40,450 & 29,950 & 30,220 \\
\hline MAX & 30,400 & 26,500 & 14,900 & 9,100 & 9,100 & 65,600 & 265,000 & 58,300 & 65,200 & 60,400 & 37,700 & 38,200 \\
\hline MIN & 24,000 & 14,200 & 4,300 & 6,500 & 7,200 & 7,700 & 46,800 & 25,600 & 24,400 & 26,500 & 26,500 & 27,800 \\
\hline AC-FT & $1,619,000$ & $1,056,000$ & 514,100 & 491,500 & 439,300 & $1,198,000$ & $6,071,000$ & $2,395,000$ & $2,377,000$ & $2,487,000$ & $1,841,000$ & $1,798,000$ \\
\hline
\end{tabular}


Table 15. Daily discharge for the Missouri River at Pierre (station 06440000) for water years 1934-65—Continued

$[---$, no data; MAX, maximum value; MIN, minimum value; AC-FT, acre-feet $]$

\begin{tabular}{|c|c|c|c|c|c|c|c|c|c|c|c|c|}
\hline Day & Oct. & Nov. & Dec. & Jan. & Feb. & Mar. & April & May & June & July & Aug. & Sept. \\
\hline \multicolumn{13}{|c|}{$\begin{array}{c}\text { DISCHARGE, CUBIC FEET PER SECOND, WATER YEAR OCTOBER } 1950 \text { TO SEPTEMBER } 1951 \\
\text { DAILY MEAN VALUES }\end{array}$} \\
\hline 1 & 28,200 & 34,900 & 5,500 & 17,600 & 13,900 & 15,800 & 40,200 & 25,800 & 41,200 & 45,200 & 32,800 & 41,200 \\
\hline 2 & 27,100 & 35,400 & 5,500 & 17,600 & 14,200 & 15,800 & 39,600 & 25,200 & 51,800 & 41,200 & 33,600 & 53,300 \\
\hline 5 & 27,800 & 35,400 & 5,700 & 17,800 & 13,700 & 17,300 & 87,200 & 25,500 & 54,800 & 37,200 & 32,000 & 42,300 \\
\hline 6 & 29,300 & 35,400 & 6,300 & 17,600 & 13,500 & 17,300 & 109,000 & 26,800 & 57,100 & 35,800 & 31,600 & 43,500 \\
\hline 7 & 30,400 & 37,200 & 7,500 & 17,600 & 12,800 & 17,800 & 109,000 & 28,200 & 53,300 & 34,900 & 32,800 & 41,800 \\
\hline 11 & 32,400 & 33,500 & 12,500 & 16,100 & 11,900 & 16,300 & 99,600 & 31,600 & 44,600 & 36,300 & 38,200 & 43,500 \\
\hline 12 & 32,400 & 31,000 & 14,000 & 16,100 & 11,900 & 15,800 & 77,900 & 31,200 & 40,200 & 34,900 & 37,700 & 41,800 \\
\hline 13 & 32,400 & 28,500 & 15,000 & 16,300 & 11,700 & 15,100 & 74,000 & 30,000 & 36,300 & 33,600 & 36,700 & 38,600 \\
\hline 14 & 32,000 & 26,800 & 16,000 & 15,600 & 11,700 & 14,600 & 71,100 & 28,500 & 34,500 & 34,500 & 34,100 & 36,300 \\
\hline 15 & 31,600 & 25,500 & 17,500 & 15,400 & 11,700 & 13,700 & 68,300 & 27,500 & 33,600 & 37,200 & 36,700 & 35,800 \\
\hline 16 & 30,800 & 24,300 & 17,000 & 14,700 & 11,200 & 13,200 & 51,100 & 26,800 & 32,800 & 38,200 & 39,100 & 35,400 \\
\hline 17 & 30,400 & 22,500 & 16,600 & 13,700 & 11,200 & 13,000 & 40,200 & 25,800 & 33,200 & 37,200 & 41,800 & 34,900 \\
\hline 18 & 29,600 & 19,700 & 15,400 & 13,500 & 11,200 & 12,600 & 36,700 & 25,500 & 34,100 & 35,800 & 43,500 & 34,900 \\
\hline 24 & 33,600 & 5,000 & 13,200 & 12,800 & 12,800 & 10,300 & 31,600 & 31,200 & 59,600 & 34,900 & 35,800 & 32,800 \\
\hline 25 & 33,200 & 4,800 & 13,900 & 13,000 & 14,600 & 10,300 & 30,800 & 32,000 & 64,700 & 33,200 & 36,700 & 33,200 \\
\hline 26 & 32,400 & 5,000 & 14,600 & 13,200 & 14,200 & 12,000 & 30,000 & 30,400 & 61,200 & 32,800 & 37,200 & 34,500 \\
\hline 27 & 33,200 & 5,300 & 15,100 & 13,200 & 14,600 & 16,000 & 28,200 & 28,900 & 52,600 & 32,400 & 36,700 & 34,900 \\
\hline 28 & 33,200 & 5,300 & 16,100 & 13,500 & 15,400 & 20,000 & 26,800 & 28,900 & 47,800 & 32,400 & 36,300 & 36,300 \\
\hline 29 & 33,200 & 5,500 & 16,800 & 13,700 & --- & 25,000 & 26,500 & 31,600 & 47,800 & 32,400 & 35,400 & 37,200 \\
\hline 30 & 33,600 & 5,700 & 17,600 & 13,500 & --- & 40,200 & 26,500 & 37,200 & 47,800 & 32,400 & 34,500 & 41,200 \\
\hline 31 & 34,100 & -- & 17,800 & 13,700 & --- & 39,100 & -- & 39,100 & --- & 32,400 & 35,400 & $\cdots$ \\
\hline
\end{tabular}

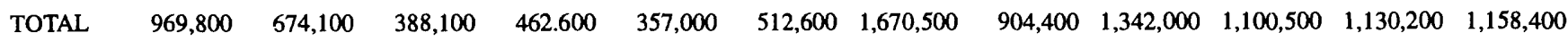

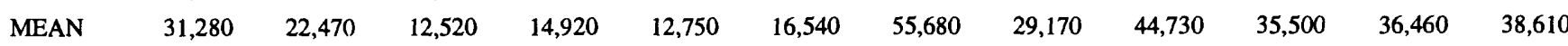
$\begin{array}{lllllllllllll}\operatorname{MAX} & 34,100 & 39,100 & 17,800 & 17,800 & 15,400 & 40,200 & 109,000 & 39,100 & 64,700 & 45,200 & 43,500 & 53,300\end{array}$

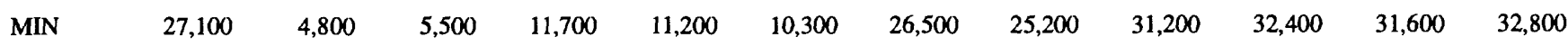

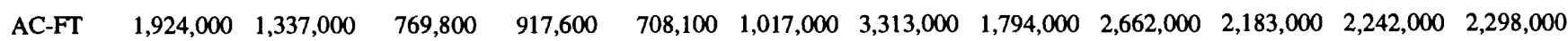


Table 15. Daily discharge for the Missouri River at Pierre (station 06440000) for water years 1934-65-Continued

[---, no data; MAX, maximum value; MIN, minimum value; AC-FT, acre-feet]

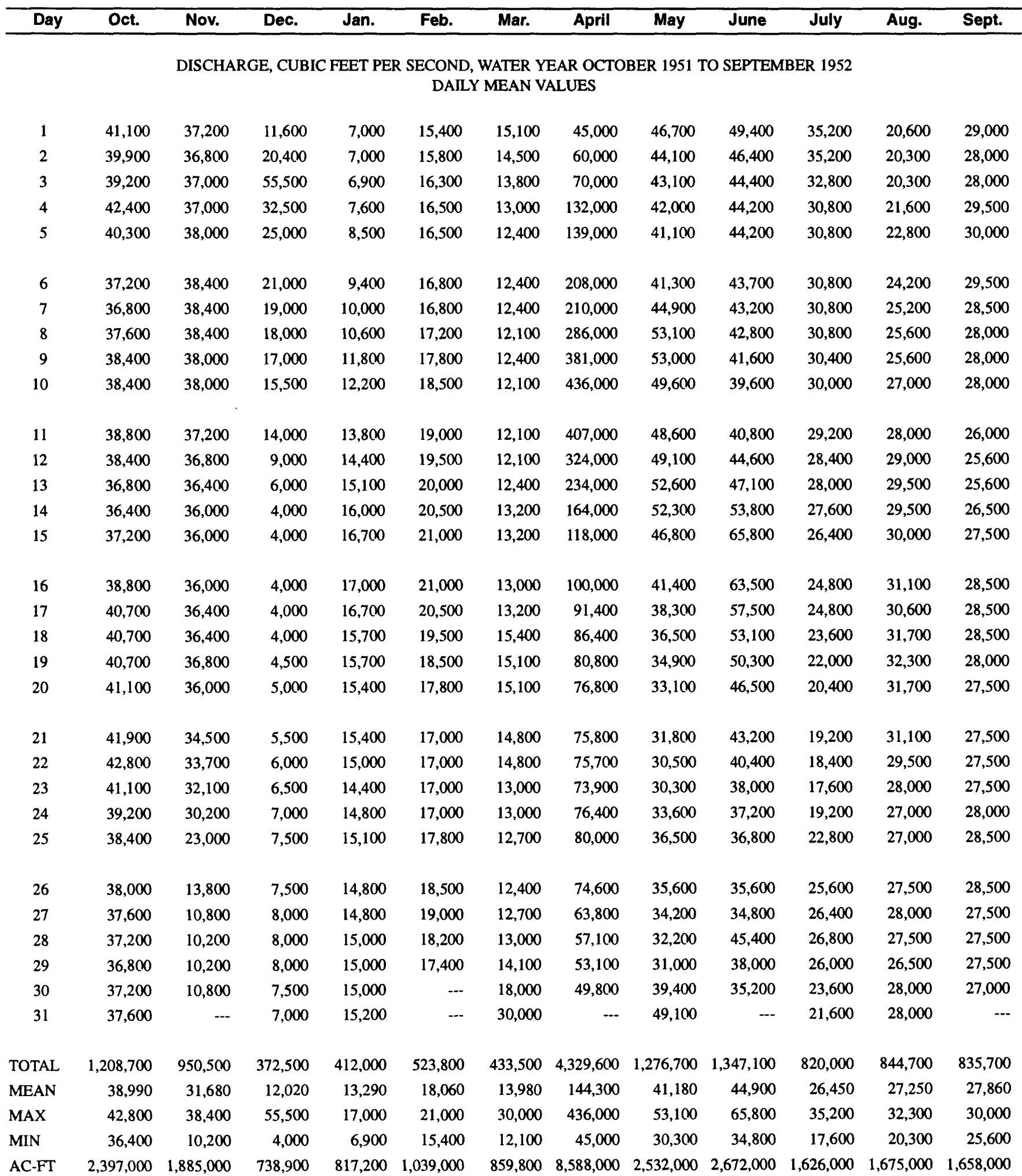


Table 15. Daily discharge for the Missouri River at Pierre (station 06440000) for water years 1934-65-Continued

[---, no data; MAX, maximum value; MIN, minimum value; AC-FT, acre-feet]

\begin{tabular}{|c|c|c|c|c|c|c|c|c|c|c|c|c|}
\hline Day & Oct. & Nov. & Dec. & Jan. & Feb. & Mar. & April & May & June & July & Aug. & Sept. \\
\hline \multicolumn{13}{|c|}{ DISCHARGE, CUBIC FEET PER SECOND, WATER YEAR OCTOBER 1952 TO SEPTEMBER 1953} \\
\hline \multicolumn{13}{|c|}{ DAILY MEAN VALUES } \\
\hline 1 & 26,700 & 25,900 & 7,000 & 12,000 & 13,000 & 11,000 & 25,200 & 29,600 & 34,700 & 54,500 & 27,900 & 27,200 \\
\hline 2 & 26,500 & 26,100 & 7,500 & 12,000 & 13,000 & 11,000 & 21,700 & 38,400 & 39,100 & 48,500 & 28,100 & 27,700 \\
\hline 3 & 26,600 & 26,400 & 8,000 & 12,000 & 13,000 & 11,000 & 21,000 & 45,000 & 33,500 & 45,600 & 31,400 & 26,500 \\
\hline 4 & 26,700 & 26,400 & 8,500 & 11,800 & 13,000 & 10,500 & 19,800 & 45,500 & 31,700 & 44,000 & 29,200 & 25,800 \\
\hline 5 & 26,900 & 26,400 & 8,500 & 11,800 & 13,000 & 10,500 & 19,700 & 40,100 & 29,800 & 42,800 & 31,400 & 26,000 \\
\hline 6 & 27,000 & 26,300 & 8,000 & 11,600 & 13,000 & 10,500 & 19,400 & 34,100 & 27,700 & 40,500 & 30,800 & 26,400 \\
\hline 7 & 27,800 & 26,100 & 8,000 & 11,200 & 13,000 & 10,500 & 24,000 & 29,900 & 43,600 & 38,300 & 30,800 & 26,700 \\
\hline 8 & 28,600 & 26,500 & 8,000 & 11,000 & 13,000 & 10,000 & 45,200 & 27,900 & 61,800 & 37,300 & 29,900 & 26,800 \\
\hline 9 & 28,900 & 27,000 & 8,500 & 10,600 & 13,000 & 10,000 & 44,600 & 28,600 & 63,900 & 36,500 & 30,000 & 27,400 \\
\hline 10 & 28,700 & 27,300 & 9,000 & 10,800 & 13,000 & 10,000 & 38,300 & 28,200 & 63,000 & 35,900 & 29,700 & 28,300 \\
\hline 11 & 28,600 & 27,300 & 8,500 & 10,800 & 12,500 & 11,000 & 27,000 & 27,900 & 60,400 & 35,400 & 29,800 & 29,100 \\
\hline 12 & 29,000 & 25,000 & 8,000 & 10,800 & 12,500 & 11,500 & 20,500 & 25,600 & 53,000 & 35,600 & 30,100 & 29,600 \\
\hline 13 & 28,900 & 23,400 & 7,000 & 11,000 & 12,500 & 12,000 & 17,400 & 24,200 & 49,300 & 35,300 & 30,100 & 30,000 \\
\hline 14 & 28,900 & 22,100 & 6,500 & 11,300 & 12,000 & 14,000 & 16,400 & 23,600 & 48,900 & 32,900 & 30,300 & 30,500 \\
\hline 15 & 29,200 & 20,700 & 6,000 & 11,200 & 12,000 & 20,000 & 15,500 & 23,500 & 56,700 & 30,500 & 30,400 & 30,600 \\
\hline 16 & 29,500 & 20,000 & 5,800 & 11,200 & 12,000 & 15,700 & 15,400 & 23,600 & 88,500 & 29,500 & 29,800 & 30,600 \\
\hline 17 & 29,400 & 19,700 & 6,000 & 11,200 & 11,500 & 16,200 & 15,500 & 24,300 & 101,000 & 29,300 & 28,600 & 29,700 \\
\hline 18 & 28,600 & 19,500 & 8,000 & 11,600 & 11,500 & 16,800 & 15,300 & 28,100 & 106,000 & 30,100 & 28,000 & 29,100 \\
\hline 19 & 28,000 & 18,700 & 10,000 & 11,600 & 12,000 & 17,500 & 14,700 & 32,300 & 79,200 & 29,600 & 27,400 & 29,100 \\
\hline 20 & 27,800 & 18,000 & 11,000 & 12,300 & 12,000 & 25,000 & 14,700 & 30,200 & 76,900 & 28,800 & 26,700 & 29,800 \\
\hline 21 & 28,000 & 17,100 & 11,500 & 12,800 & 12,000 & 32,000 & 14,400 & 26,700 & 94,800 & 27,600 & 26,700 & 31,000 \\
\hline 22 & 28,800 & 17,100 & 13,000 & 12,600 & 12,000 & 40,000 & 14,100 & 24,700 & 111,000 & 26,700 & 25,900 & 30,400 \\
\hline 23 & 29,100 & 16,900 & 14,000 & 12,300 & 11,500 & 43,400 & 13,900 & 22,700 & 102,000 & 25,800 & 26,000 & 29,500 \\
\hline 24 & 29,500 & 16,800 & 14,500 & 12,300 & 11,500 & 39,000 & 13,600 & 21,600 & 90,600 & 24,700 & 26,400 & 30,000 \\
\hline 25 & 29,400 & 16,100 & 14,500 & 12,900 & 12,000 & 34,300 & 13,000 & 20,900 & 85,200 & 24,800 & 27,300 & 30,500 \\
\hline 26 & 29,100 & 15,800 & 14,000 & 13,000 & 11,500 & 28,800 & 13,400 & 21,900 & 82,800 & 27,200 & 27,400 & 30,400 \\
\hline 27 & 28,200 & 12,000 & 13,000 & 13,600 & 11,000 & 25,500 & 14,400 & 23,500 & 80,600 & 28,200 & 28,400 & 30,300 \\
\hline 28 & 27,500 & 9,000 & 12,000 & 13,800 & 11,000 & 24,600 & 15,300 & 23,500 & 73,200 & 28,900 & 29,600 & 30,200 \\
\hline 29 & 26,200 & 8,000 & 11,500 & 13,500 & -- & 24,600 & 16,600 & 24,000 & 66,000 & 28,400 & 28,800 & 30,200 \\
\hline 30 & 25,700 & 7,000 & 11,200 & 13,200 & --. & 26,700 & 20,200 & 23,200 & 59,900 & 29,500 & 27,600 & 30,400 \\
\hline 31 & 25,600 & --- & 11,000 & 13,000 &..- & 38,500 & --. & 23,500 & $\ldots$ & 30,100 & 26,900 & $\ldots$ \\
\hline
\end{tabular}

\begin{tabular}{|c|c|c|c|c|c|c|c|c|c|c|c|c|}
\hline TOTAL & 869,400 & 614,600 & 298,000 & 370,800 & 343,000 & 622,100 & 600,200 & 866,800 & $1,994,800$ & $1,042,800$ & 891,400 & 869,800 \\
\hline MEAN & 28,050 & 20,490 & 9,613 & 11,960 & 12,250 & 20,070 & 20,010 & 27,960 & 66,490 & 33,640 & 28,750 & 28,990 \\
\hline MAX & 29,500 & 27,300 & 14,500 & 13,800 & 13,000 & 43,400 & 45,200 & 45,500 & 111,000 & 54,500 & 31,400 & 31,000 \\
\hline MIN & 25,600 & 7,000 & 5,800 & 10,600 & 11,000 & 10,000 & 13,000 & 20,900 & 27,700 & 24,700 & 25,900 & 25,800 \\
\hline AC-FT & $1,724,000$ & $1,219,000$ & 591,100 & 735,500 & 680,300 & $1,234,000$ & $1,190,000$ & $1,719,000$ & $3,957,000$ & $2,068,000$ & $1,768,000$ & $1,725,000$ \\
\hline
\end{tabular}


Table 15. Daily discharge for the Missouri River at Pierre (station 06440000) for water years 1934-65-Continued [---, no data; MAX, maximum value; MIN, minimum value; AC-FT, acre-feet]

\begin{tabular}{|c|c|c|c|c|c|c|c|c|c|c|c|c|}
\hline Day & Oct. & Nov. & Dec. & Jan. & Feb. & Mar. & April & May & June & July & Aug. & Sept. \\
\hline \multicolumn{13}{|c|}{$\begin{array}{l}\text { DISCHARGE, CUBIC FEET PER SECOND, WATER YEAR OCTOBER } 1953 \text { TO SEPTEMBER } 1954 \\
\text { DAILY MEAN VALUES }\end{array}$} \\
\hline 1 & 30,400 & 37,500 & 16,400 & 8,500 & 9,000 & 18,600 & 14,700 & 28,200 & 28,000 & 13,700 & 39,100 & 39,000 \\
\hline 2 & 30,800 & 34,600 & 16,000 & 8,500 & 9,000 & 17,000 & 13,800 & 28,200 & 28,700 & 14,000 & 39,600 & 38,800 \\
\hline 3 & 31,100 & 32,900 & 15,900 & 8,500 & 9,000 & 15,000 & 13,300 & 27,600 & 27,000 & 14,200 & 39,500 & 38,800 \\
\hline 4 & 31,100 & 32,200 & 15,500 & 9,000 & 9,500 & 13,000 & 12,800 & 27,400 & 25,900 & 14,400 & 39,200 & 39,100 \\
\hline 5 & 31,100 & 31,800 & 15,300 & 9,000 & 9,800 & 13,000 & 12,500 & 27,600 & 25,300 & 14,500 & 38,500 & 39,700 \\
\hline 6 & 31,200 & 31,600 & 15,200 & 9,000 & 10,000 & 22,000 & 13,600 & 27,300 & 25,600 & 14,300 & 38,000 & 40,400 \\
\hline 7 & 31,600 & 31,300 & 13,500 & 8,000 & 10,200 & 20,000 & 13,900 & 26,500 & 25,600 & 14,700 & 37,600 & 40,200 \\
\hline 8 & 31,500 & 30,800 & 12,900 & 7,500 & 10,300 & 20,400 & 17,000 & 26,100 & 25,500 & 16,500 & 38,000 & 40,400 \\
\hline 9 & 31,300 & 30,200 & 12,600 & 7,500 & 10,500 & 19,700 & 27,400 & 25,700 & 26,200 & 20,300 & 38,300 & 39,300 \\
\hline 10 & 31,200 & 29,700 & 12,100 & 6,000 & 10,600 & 20,500 & 27,400 & 25,700 & 28,500 & 23,300 & 38,800 & 39,600 \\
\hline 11 & 31,200 & 29,500 & 10,600 & 6,500 & 10,600 & 20,100 & 25,000 & 25,600 & 29,600 & 24,600 & 39,600 & 39,600 \\
\hline 12 & 31,800 & 29,000 & 10,400 & 7,000 & 11,000 & 18,000 & 25,500 & 25,300 & 35,200 & 24,200 & 39,400 & 39,800 \\
\hline 13 & 32,000 & 28,000 & 9,800 & 7,500 & 11,000 & 17,000 & 29,100 & 24,600 & 33,200 & 24,200 & 39,300 & 39,200 \\
\hline 14 & 32,100 & 26,400 & 10,300 & 7,800 & 10,500 & 15,000 & 30,800 & 23,400 & 31,200 & 24,300 & 39,600 & 38,700 \\
\hline 15 & 31,800 & 24,500 & 10,500 & 9,500 & 10,400 & 14,000 & 29,800 & 23,400 & 28,800 & 25,700 & 40,300 & 38,500 \\
\hline 16 & 31,500 & 23,400 & 10,000 & 9,600 & 10,200 & 14,000 & 28,200 & 23,400 & 25,200 & 27,800 & 40,600 & 38,800 \\
\hline 17 & 31,200 & 22,400 & 6,500 & 10,000 & 10,000 & 14,900 & 26,500 & 23,600 & 21,100 & 29,000 & 40,000 & 38,900 \\
\hline 18 & 31,500 & 21,800 & 6,000 & 10,500 & 10,000 & 15,200 & 25,700 & 24,000 & 18,100 & 28,900 & 39,000 & 39,200 \\
\hline 19 & 32,000 & 20,900 & 6,000 & 10,000 & 10,000 & 18,000 & 26,400 & 24,100 & 16,200 & 29,000 & 38,800 & 39,100 \\
\hline 20 & 32,800 & 19,100 & 5,500 & 9,300 & 12,600 & 22,200 & 27,000 & 23,700 & 14,800 & 31,000 & 39,700 & 39,200 \\
\hline 21 & 34,100 & 19,200 & 5,500 & 9,000 & 12,800 & 24,800 & 27,200 & 23,400 & 14,500 & 34,000 & 40,000 & 38,900 \\
\hline 22 & 33,500 & 17,900 & 5,500 & 9,000 & 12,900 & 24,700 & 27,100 & 23,700 & 14,000 & 34,700 & 40,500 & 38,800 \\
\hline 23 & 32,300 & 17,400 & 5,500 & 9,000 & 11,400 & 25,200 & 27,100 & 24,100 & 13,600 & 34,500 & 39,200 & 39,200 \\
\hline 24 & 31,300 & 17,300 & 5,500 & 8,800 & 14,400 & 26,000 & 27,100 & 24,300 & 13,300 & 34,600 & 38,200 & 39,100 \\
\hline 25 & 31,700 & 17,000 & 7,500 & 8,500 & 14,800 & 26,900 & 27,300 & 24,800 & 13,600 & 35,000 & 38,900 & 39,000 \\
\hline 26 & 32,700 & 17,100 & 6,500 & 8,800 & 14,000 & 24,800 & 26,800 & 26,300 & 13,200 & 35,400 & 39,300 & 39,000 \\
\hline 27 & 33,700 & 17,400 & 6,000 & 8,800 & 13,700 & 21,100 & 26,400 & 26,900 & 12,600 & 35,600 & 39,000 & 38,800 \\
\hline 28 & 37,100 & 16,900 & 7,500 & 9,000 & 14,700 & 19,300 & 26,700 & 26,500 & 12,400 & 36,700 & 38,900 & 38,600 \\
\hline 29 & 46,200 & 16,800 & 8,500 & 9,000 & --. & 18,000 & 27,100 & 26,500 & 13,000 & 38,300 & 38,800 & 38,200 \\
\hline 30 & 43,800 & 16,500 & 8,200 & 9,000 & --- & 16,800 & 28,100 & 26,700 & 13,600 & 38,600 & 38,800 & 38,400 \\
\hline 31 & 41,000 & -.- & 7,800 & 9,000 & --- & 16,000 & -.. & 27,400 & -. & 38,400 & 38,700 & -.. \\
\hline TOTAL & $1,026,600$ & 741,100 & 305,000 & 267,100 & 312,900 & 591,200 & 711,300 & 792,000 & 653,500 & 824,400 & $1,213,200$ & $1,174,300$ \\
\hline MEAN & 33,120 & 24,700 & 9,839 & 8,616 & 11,170 & 19,070 & 23,710 & 25,550 & 21,780 & 26,590 & 39,140 & 39,140 \\
\hline MAX & 46,200 & 37,500 & 16,400 & 10,500 & 14,800 & 26,900 & 30,800 & 28,200 & 35,200 & 38,600 & 40,600 & 40,400 \\
\hline MIN & 30,400 & 16,500 & 5,500 & 6,000 & 9,000 & 13,000 & 12,500 & 23,400 & 12,400 & 13,700 & 37,600 & 38,200 \\
\hline AC-FT & $2,036,000$ & $1,470,000$ & 605,000 & 529,800 & 620,600 & $1,173,000$ & $1,411,000$ & $1,571,000$ & $1,296,000$ & $1,635,000$ & $2,406,000$ & $2,329,000$ \\
\hline
\end{tabular}


Table 15. Daily discharge for the Missouri River at Pierre (station 06440000) for water years 1934-65—Continued

[---, no data; MAX, maximum value; MIN, minimum value; AC-FT, acre-feet]

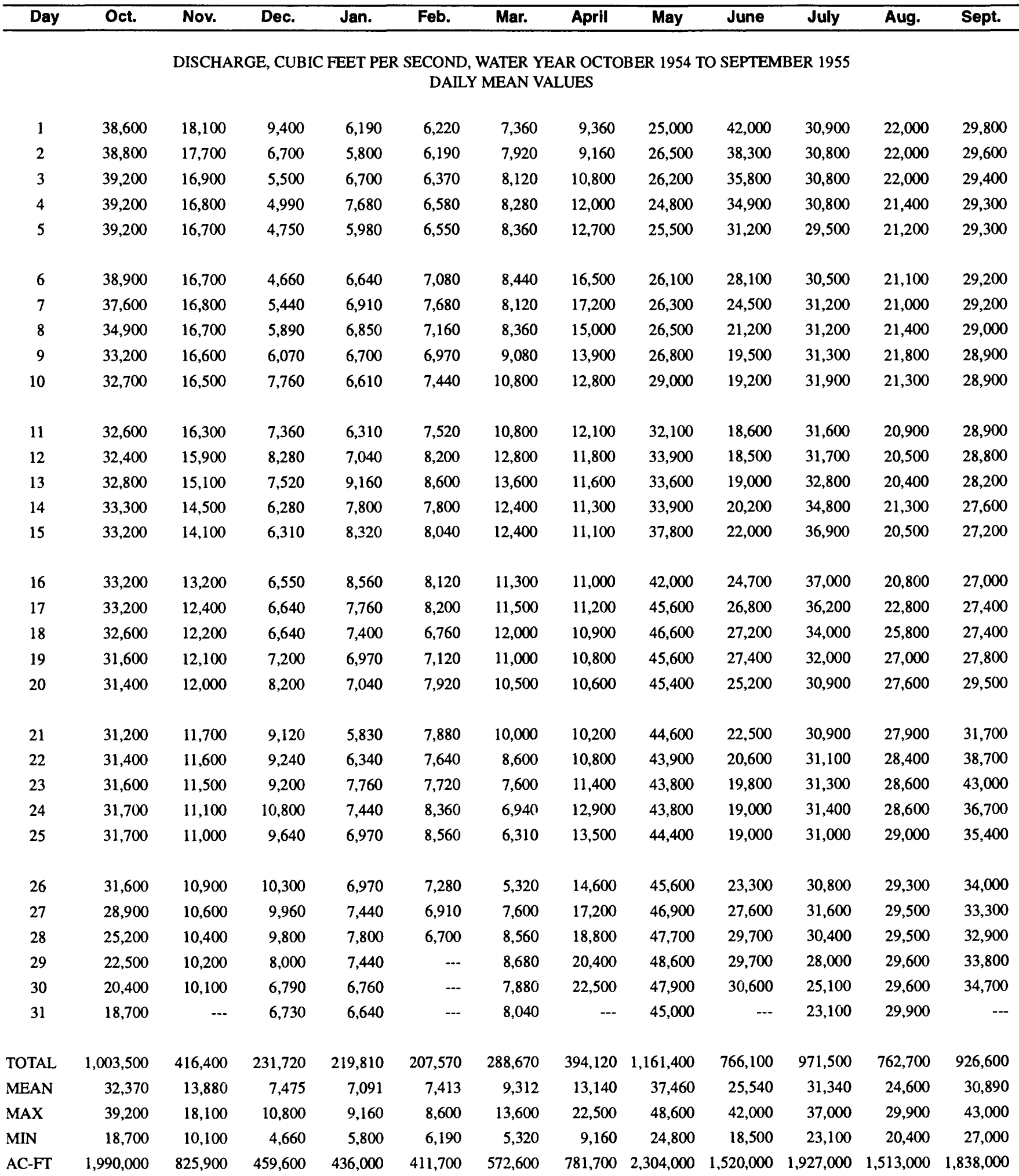


Table 15. Daily discharge for the Missouri River at Pierre (station 06440000) for water years 1934-65-Continued [---, no data; MAX, maximum value; MIN, minimum value; AC-FT, acre-feet]

\begin{tabular}{|c|c|c|c|c|c|c|c|c|c|c|c|c|}
\hline Day & Oct. & Nov. & Dec. & Jan. & Feb. & Mar. & April & May & June & July & Aug. & Sept. \\
\hline \multicolumn{13}{|c|}{$\begin{array}{c}\text { DISCHARGE, CUBIC FEET PER SECOND, WATER YEAR OCTOBER } 1955 \text { TO SEPTEMBER } 1956 \\
\text { DAILY MEAN VALUES }\end{array}$} \\
\hline 1 & 33,600 & 32,500 & 8,800 & 10,500 & 8,000 & 6,300 & 25,300 & 25,600 & 31,800 & 35,600 & 27,600 & 24,200 \\
\hline 2 & 32,900 & 33,100 & 6,500 & 10,400 & 7,400 & 6,200 & 29,600 & 25,600 & 31,700 & 34,400 & 28,300 & 23,600 \\
\hline 3 & 31,900 & 32,800 & 7,000 & 10,300 & 7,600 & 6,300 & 31,200 & 26,200 & 31,500 & 33,100 & 30,600 & 22,500 \\
\hline 4 & 31,700 & 32,600 & 7,400 & 10,300 & 7,600 & 6,800 & 26,500 & 28,200 & 30,800 & 31,900 & 31,200 & 20,400 \\
\hline 5 & 31,900 & 32,400 & 7,200 & 10,500 & 7,500 & 6,900 & 27,400 & 29,500 & 30,500 & 32,200 & 30,900 & 20,300 \\
\hline 6 & 31,800 & 32,000 & 7,000 & 10,400 & 7,500 & 6,600 & 34,400 & 29,100 & 31,000 & 33,100 & 30,700 & 20,300 \\
\hline 7 & 31,700 & 31,700 & 6,800 & 9,900 & 8,000 & 6,600 & 36,800 & 28,100 & 30,900 & 35,700 & 31,400 & 20,700 \\
\hline & 31,600 & 31,400 & 7,000 & 9,900 & 7,800 & 7,000 & 32,800 & 27,800 & 31,000 & 34,800 & 31,600 & 21,300 \\
\hline \multirow[t]{2}{*}{. } & 31,400 & 28,700 & 6,900 & 9,800 & 8,000 & 7,200 & 34,100 & 27,400 & 32,700 & 32,800 & 31,900 & 21,800 \\
\hline & 31,400 & 26,700 & 7,400 & 9,700 & 7,200 & 7,400 & 35,600 & 27,300 & 33,000 & 32,400 & 32,400 & 22,000 \\
\hline 11 & 32,000 & 25.000 & 8,100 & 9,400 & 6,600 & 7,600 & 41,700 & 27,700 & 31,700 & 33,400 & 31,100 & 22,800 \\
\hline 12 & 32,200 & 24,200 & 8,200 & 9,300 & 6,400 & 7,500 & 35,000 & 27,900 & 31,500 & 32,600 & 31,900 & 22,500 \\
\hline 13 & 32,300 & 20,400 & 7,900 & 9,200 & 6,600 & 7,400 & 26,500 & 29,200 & 32,000 & 31,700 & 31,200 & 22,400 \\
\hline 14 & 31,400 & 20,000 & 7,800 & 8,900 & 6,000 & 7,300 & 25,800 & 30,000 & 32,000 & 30,900 & 31,000 & 22,700 \\
\hline 15 & 31,500 & 17,100 & 7,800 & 8,400 & 5,500 & 7,400 & 26,900 & 30,200 & 32,900 & 30,700 & 31,000 & 22,400 \\
\hline 16 & 31,600 & 13,400 & 8,000 & 7,900 & 5,600 & 7,600 & 26,700 & 30,300 & 34,200 & 30,800 & 30,800 & 22,200 \\
\hline 17 & 31,600 & 12,600 & 8,300 & 7,800 & 5,300 & 8,800 & 26,400 & 29,400 & 35,000 & 31,700 & 30,600 & 22,100 \\
\hline 18 & 31,800 & 10,900 & 8,900 & 8,300 & 5,400 & 10,500 & 26,300 & 28,600 & 34,500 & 32,200 & 30,200 & 21,900 \\
\hline 19 & 32,000 & 11,000 & 9,500 & 8,400 & 5,100 & 12,000 & 25,900 & 28,400 & 34,000 & 31,400 & 30,000 & 21,900 \\
\hline 20 & 32,000 & 11,000 & 10,000 & 8,100 & 4,800 & 22,000 & 25,600 & 27,900 & 35,000 & 30,800 & 29,600 & 22,500 \\
\hline 21 & 31,900 & 12,900 & 10,000 & 7,900 & 5,000 & 27,000 & 25,600 & 27,000 & 37,100 & 30,600 & 28,000 & 22,400 \\
\hline 22 & 31,800 & 12,800 & 10,000 & 7,800 & 5,400 & 32,000 & 25,300 & 26,800 & 36,700 & 30,500 & 26,000 & 21,500 \\
\hline 23 & 32,100 & 11,600 & 10,000 & 7,700 & 5,800 & 38,000 & 24,800 & 27,100 & 36,300 & 30,300 & 25,500 & 19,900 \\
\hline 24 & 32,300 & 11,000 & 9,900 & 7,700 & 6,000 & 46,000 & 24,900 & 27,600 & 36,400 & 30,000 & 25,800 & 18,600 \\
\hline 25 & 32,400 & 10,500 & 9,900 & 7,700 & 6,400 & 58,700 & 24,900 & 27,900 & 37,000 & 29,800 & 25,400 & 17,900 \\
\hline 26 & 32,700 & 10,000 & 10,100 & 7,800 & 6,400 & 45,700 & 25,200 & 27,900 & 37,800 & 29,400 & 24,900 & 17,900 \\
\hline 27 & 33,000 & 9,700 & 10,400 & 8,000 & 6,800 & 39,000 & 26,400 & 28,100 & 38,200 & 29,100 & 24,500 & 17,800 \\
\hline 28 & 33,200 & 9,500 & 10,300 & 8,000 & 6,300 & 35,700 & 26,200 & 29,300 & 37,600 & 29,500 & 24,800 & 17,400 \\
\hline 29 & 33,100 & 9,000 & 10,300 & 8,000 & 6,400 & 25,500 & 26,200 & 30,700 & 36,500 & 29,600 & 24,900 & 17,400 \\
\hline 30 & 32,800 & 9,000 & 10,400 & 8,000 & --- & 21,600 & 25,600 & 31,900 & 35,800 & 30,300 & 25,100 & 17,500 \\
\hline 31 & 32,300 & --- & 10,500 & 8,000 & --- & 21,800 & -- & 32,200 & --- & 28,700 & 24,800 & --- \\
\hline TOTAL & 995,900 & 585,500 & 268,300 & 274,000 & 188,400 & 556,400 & 855,600 & 880,900 & $1,017,100$ & 980,000 & 893,700 & 628,800 \\
\hline MEAN & 32,130 & 19,520 & 8,655 & 8,839 & 6,497 & 17,950 & 28,520 & 28,420 & 33,900 & 31,610 & 28,830 & 20,960 \\
\hline MAX & 33,600 & 33,100 & 10,500 & 10,500 & 8,000 & 58,700 & 41,700 & 32,200 & 38,200 & 35,700 & 32,400 & 24,200 \\
\hline MIN & 31,400 & 9,000 & 6,500 & 7,700 & 4,800 & 6,200 & 24,800 & 25,600 & 30,500 & 28,700 & 24,500 & 17,400 \\
\hline AC-FT & $1,975,000$ & $1,161,000$ & 532,200 & 543,500 & 373,700 & $1,104,000$ & $1,697,000$ & $1,747,000$ & $2,017,000$ & $1,944,000$ & $1,773,000$ & $1,247,000$ \\
\hline
\end{tabular}


Table 15. Daily discharge for the Missouri River at Pierre (station 06440000) for water years 1934-65-Continued [---, no data; MAX, maximum value; MIN, minimum value; AC-FT, acre-feet]

\begin{tabular}{|c|c|c|c|c|c|c|c|c|c|c|c|c|}
\hline Day & Oct. & Nov. & Dec. & Jan. & Feb. & Mar. & April & May & June & July & Aug. & Sept. \\
\hline \multicolumn{13}{|c|}{$\begin{array}{l}\text { DISCHARGE, CUBIC FEET PER SECOND, WATER YEAR OCTOBER } 1956 \text { TO SEPTEMBER } 1957 \\
\text { DAILY MEAN VALUES }\end{array}$} \\
\hline 1 & 17,900 & 16,500 & 13,000 & 11,500 & 12,000 & 13,500 & 16,500 & 17,100 & 25,600 & 21,000 & 15,700 & 24,400 \\
\hline 2 & 17,700 & 16,800 & 13,300 & 11,000 & 12,000 & 14,000 & 17,600 & 16,000 & 22,900 & 19,500 & 16,000 & 21,500 \\
\hline 3 & 17,300 & 16,600 & 13,700 & 11,000 & 12,500 & 14,000 & 17,600 & 14,200 & 20,600 & 19,400 & 16,000 & 20,800 \\
\hline 4 & 16,800 & 16,800 & 14,400 & 11,000 & 12,500 & 14,000 & 17,300 & 13,200 & 17,700 & 21,200 & 15,500 & 18,900 \\
\hline 5 & 15,900 & 17,000 & 13,200 & 11,000 & 13,000 & 14,000 & 16,900 & 14,600 & 15,000 & 20,700 & 15,400 & 18,300 \\
\hline 6 & 15,800 & 16,700 & 10,300 & 11,000 & 13,000 & 14,000 & 18,000 & 16,800 & 13,600 & 18,100 & 15,800 & 17,900 \\
\hline 7 & 15,600 & 16,400 & 6,460 & 10,500 & 13,500 & 14,000 & 17,800 & 17,600 & 13,100 & 17,200 & 17,600 & 16,600 \\
\hline 8 & 15,200 & 16,400 & 7,000 & 10,000 & 13,500 & 14,500 & 19,400 & 17,300 & 12,400 & 17,900 & 19,200 & 16,200 \\
\hline 9 & 14,700 & 16,200 & 8,000 & 10,000 & 13,500 & 15,000 & 19,400 & 16,900 & 12,300 & 18,000 & 20,000 & 17,200 \\
\hline 10 & 14,500 & 16,300 & 7,000 & 10,000 & 13,500 & 15,000 & 19,500 & 16,200 & 14,300 & 18,000 & 20,000 & 18,000 \\
\hline 11 & 14,600 & 16,400 & 6,500 & 10,000 & 13,500 & 15,000 & 19,100 & 15,800 & 18,600 & 17,900 & 18,900 & 17,700 \\
\hline 12 & 14,600 & 16,400 & 6,500 & 10,000 & 13,500 & 15,000 & 19,100 & 15,800 & 23,400 & 17,800 & 19,100 & 17,400 \\
\hline 13 & 15,100 & 16,200 & 6,500 & 10,000 & 13,500 & 15,000 & 19,400 & 16,600 & 23,600 & 17,300 & 19,200 & 16,700 \\
\hline 14 & 15,600 & 15,200 & 7,500 & 10,000 & 14,000 & 15,000 & 19,100 & 17,700 & 19,400 & 16,700 & 18,500 & 15,400 \\
\hline 15 & 16,400 & 14,200 & 8,500 & 10,000 & 13,500 & 15,000 & 19,300 & 18,700 & 17,000 & 16,000 & 18,700 & 15,400 \\
\hline 16 & 16,600 & 13,200 & 9,500 & 10,000 & 13,000 & 16,000 & 19,200 & 21,700 & 19,800 & 15,700 & 21,300 & 15,800 \\
\hline 17 & 16,600 & 12,500 & 11,000 & 10,000 & 13,000 & 15,500 & 19,100 & 25,700 & 23,100 & 15,800 & 23,800 & 14,900 \\
\hline 18 & 16,400 & 12,700 & 12,000 & 10,000 & 13,000 & 15,000 & 20,100 & 26,300 & 22,200 & 15,700 & 24,800 & 14,400 \\
\hline 19 & 16,200 & 12,700 & 11,000 & 10,000 & 13,000 & 15,000 & 21,300 & 25,400 & 21,900 & 16,800 & 24,300 & 15,500 \\
\hline 20 & 16,300 & 13,000 & 10,500 & 9,500 & 13,000 & 15,000 & 24,300 & 26,400 & 21,300 & 17,600 & 23,900 & 17,600 \\
\hline 21 & 16,300 & 13,200 & 10,000 & 9,500 & 13,500 & 15,500 & 23,300 & 31,300 & 20,200 & 17,900 & 23,700 & 18,300 \\
\hline 22 & 16,100 & 13,400 & 10,500 & 9,500 & 13,500 & 15,500 & 21,100 & 32,400 & 18,600 & 18,800 & 24,000 & 18,900 \\
\hline 23 & 15,900 & 13,800 & 12,000 & 9,500 & 13,500 & 15,000 & 21,200 & 32,100 & 18,300 & 18,200 & 24,400 & 19,700 \\
\hline 24 & 15,400 & 14,000 & 12,000 & 10,000 & 13,500 & 13,800 & 19,700 & 31,200 & 19,200 & 16,600 & 24,500 & 19,900 \\
\hline 25 & 15,100 & 14,400 & 11,000 & 10,500 & 13,500 & 13,000 & 17,000 & 33,700 & 20,300 & 15,900 & 24,800 & 20,000 \\
\hline 26 & 15,800 & 14,600 & 11,000 & 10,500 & 13,500 & 13,200 & 15,100 & 52,500 & 21,600 & 16,800 & 25,000 & 19,800 \\
\hline 27 & 16,100 & 14,200 & 11,000 & 10,500 & 13,500 & 14,000 & 14,200 & 61,400 & 21,900 & 18,500 & 24,900 & 19,300 \\
\hline 28 & 16,400 & 14,100 & 10,500 & 10,500 & 13,500 & 14,600 & 15,400 & 44,900 & 20,700 & 19,500 & 24,200 & 17,700 \\
\hline 29 & 16,800 & 14,000 & 10,500 & 10,500 & -.. & 15,500 & 17,000 & 34,100 & 20,100 & 20,100 & 24,200 & 16,000 \\
\hline 30 & 17,000 & 13,200 & 11,000 & 11,000 & -.. & 16,000 & 17,900 & 33,000 & 21,000 & 19,200 & 24,800 & 14,200 \\
\hline 31 & 16,800 & --. & 12,000 & 11,500 & -.- & 15,600 & -.. & 29,200 & -.- & 16,400 & 25,800 & ... \\
\hline TOTAL & 497,500 & 447,100 & 317,360 & 320,000 & 370,000 & 455,200 & 561,900 & 785,800 & 579,700 & 556,200 & 654,000 & 534,400 \\
\hline MEAN & 16,050 & 14,900 & 10,240 & 10,320 & 13,210 & 14,680 & 18,730 & 25,350 & 19,320 & 17,940 & 21,100 & 17,810 \\
\hline MAX & 17,900 & 17,000 & 14,400 & 11,500 & 14,000 & 16,000 & 24,300 & 61,400 & 25,600 & 21,200 & 25,800 & 24,400 \\
\hline MIN & 14,500 & 12,500 & 6,460 & 9,500 & 12,000 & 13,000 & 14,200 & 13,200 & 12,300 & 15,700 & 15,400 & 14,200 \\
\hline AC-FT & 986,800 & 886,800 & 629,500 & 634,700 & 733,900 & 902,900 & $1,115,000$ & $1,559,000$ & $1,150,000$ & $1,103,000$ & $1,297,000$ & $1,060,000$ \\
\hline
\end{tabular}


Table 15. Daily discharge for the Missouri River at Pierre (station 06440000) for water years 1934-65—Continued [---, no data; MAX, maximum value; MIN, minimum value; AC-FT, acre-feet $]$

\begin{tabular}{|c|c|c|c|c|c|c|c|c|c|c|c|c|}
\hline Day & Oct. & Nov. & Dec. & Jan. & Feb. & Mar. & April & May & June & July & Aug. & Sept. \\
\hline \multicolumn{13}{|c|}{$\begin{array}{l}\text { DISCHARGE, CUBIC FEET PER SECOND, WATER YEAR OCTOBER } 1957 \text { TO SEPTEMBER } 1958 \\
\text { DAILY MEAN VALUES }\end{array}$} \\
\hline 1 & 13,300 & 16,600 & 10,800 & 4,500 & 15,200 & 16,600 & 32,800 & 21,000 & 18,600 & 30,400 & 9,760 & 22,500 \\
\hline 2 & 13,100 & 16,300 & 11,200 & 4,500 & 15,200 & 15,500 & 41,600 & 21,100 & 18,800 & 30,800 & 7,930 & 23,200 \\
\hline 3 & 12,800 & 15,100 & 11,200 & 4,800 & 14,500 & 15,800 & 38,100 & 21,200 & 19,400 & 31,800 & 1,230 & 24,000 \\
\hline 4 & 12,200 & 12,100 & 10,500 & 5,500 & 13,500 & 13,000 & 35,700 & 21,600 & 20,000 & 32,300 & 2,170 & 24,600 \\
\hline 5 & 11,600 & 10,600 & 10,700 & 6,700 & 13,500 & 11,600 & 36,300 & 21,300 & 20,600 & 31,500 & 2,860 & 25,200 \\
\hline 6 & 11,700 & 10,200 & 11,200 & 6,500 & 14,000 & 11,000 & 31,100 & 21,200 & 23,600 & 31,100 & 3,540 & 25,800 \\
\hline 7 & 11,800 & 9,880 & 11,500 & 7,000 & 15,000 & 13,000 & 29,500 & 21,400 & 23,900 & 28,600 & 4,120 & 26,200 \\
\hline 8 & 12,100 & 9,790 & 11,000 & 8,000 & 15,000 & 14,000 & 27,700 & 21,600 & 24,100 & 26,300 & 4,560 & 27,000 \\
\hline 9 & 12,800 & 9,730 & 10,700 & 9,000 & 14,700 & 14,500 & 25,800 & 21,500 & 25,800 & 22,600 & 4,900 & 27,300 \\
\hline 10 & 13,600 & 9,400 & 11,100 & 10,000 & 14,200 & 15,000 & 24,100 & 21,300 & 29,700 & 19,800 & 5,360 & 26,800 \\
\hline 11 & 13,900 & 9,250 & 11,600 & 12,000 & 14,500 & 15,200 & 22,200 & 21,400 & 34,500 & 18,100 & 5,680 & 27,800 \\
\hline 12 & 13,800 & 9,310 & 11,200 & 13,500 & 13,500 & 15,300 & 19,600 & 21,300 & 34,300 & 16,800 & 5,820 & 26,800 \\
\hline 13 & 14,100 & 9,280 & 10,300 & 13,000 & 12,500 & 15,500 & 17,200 & 21,800 & 34,200 & 16,100 & 5,930 & 28,100 \\
\hline 14 & 15,100 & 9,160 & 8,410 & 12,500 & 12,000 & 15,500 & 16,200 & 22,900 & 34,000 & 15,400 & 6,090 & 28,100 \\
\hline 15 & 16,000 & 9,130 & 7,840 & 14,000 & 11,700 & 15,700 & 16,400 & 23,700 & 34,700 & 15,400 & 6,170 & 28,100 \\
\hline 16 & 16,200 & 9,010 & 7,630 & 16,000 & 11,500 & 16,000 & 16,900 & 24,000 & 34,900 & 15,100 & 6,280 & 28,200 \\
\hline 17 & 15,500 & 8,830 & 9,250 & 17,100 & 11,800 & 15,700 & 17,200 & 22,900 & 33,800 & 14,900 & 6,510 & 28,500 \\
\hline 18 & 14,200 & 8,560 & 11,100 & 17,500 & 12,000 & 15,500 & 16,900 & 22,000 & 36,000 & 14,800 & 7,190 & 28,500 \\
\hline 19 & 12,900 & 8,590 & 12,500 & 17,500 & 12,200 & 15,500 & 16,300 & 21,600 & 34,800 & 14,900 & 8,230 & 28,700 \\
\hline 20 & 12,900 & 8,620 & 12,600 & 16,500 & 12,600 & 15,500 & 16,200 & 21,400 & 33,400 & 14,900 & 9,490 & 28,600 \\
\hline 21 & 13,500 & 8,620 & 11,800 & 16,000 & 12,800 & 15,300 & 16,500 & 21,400 & 33,400 & 14,700 & 10,700 & 28,700 \\
\hline 22 & 14,100 & 8,500 & 11,000 & 15,500 & 13,200 & 15,000 & 17,400 & 21,200 & 32,900 & 15,100 & 11,900 & 28,900 \\
\hline 23 & 14,600 & 8,590 & 10,500 & 15,300 & 13,700 & 15,300 & 17,700 & 21,000 & 32,400 & 15,900 & 12,900 & 29,100 \\
\hline 24 & 15,100 & 10,000 & 11,000 & 15,500 & 14,200 & 16,000 & 18,500 & 20,700 & 32,700 & 14,800 & 13,700 & 29,100 \\
\hline 25 & 15,400 & 11,300 & 10,500 & 16,000 & 14,500 & 16,400 & 20,000 & 20,800 & 32,000 & 14,500 & 14,800 & 29,200 \\
\hline 26 & 15,500 & 11,900 & 10,500 & 15,800 & 14,800 & 17,500 & 20,900 & 20,800 & 31,100 & 14,400 & 16,000 & 29,400 \\
\hline 27 & 15,100 & 12,300 & 10,500 & 15,500 & 16,000 & 18,500 & 21,500 & 20,400 & 31,500 & 14,500 & 17,300 & 29,600 \\
\hline 28 & 15,300 & 12,400 & 10,000 & 15,000 & 17,000 & 22,000 & 22,700 & 20,600 & 31,300 & 13,500 & 18,600 & 29,900 \\
\hline 29 & 15,200 & 11,900 & 9.000 & 14,300 & -.. & 35,000 & 22,400 & 20,600 & 30,600 & 12,800 & 19,800 & 29,900 \\
\hline 30 & 15,100 & 11,400 & 7,000 & 14,300 & --- & 49,100 & 21,600 & 19,800 & 30,600 & 12,200 & 20,000 & 29,900 \\
\hline 31 & 16,000 & -- & 5,000 & 14,800 & -- & 28,900 & -- & 19,400 & $\ldots$ & 11,200 & 21,700 & --- \\
\hline TOTAL & 434,500 & 316,350 & 319,130 & 384,100 & 385,300 & 544,400 & 697,000 & 662,900 & 887,600 & 595,200 & 291,220 & 827,700 \\
\hline MEAN & 14,020 & 10,540 & 10,290 & 12,390 & 13,760 & 17,560 & 23,230 & 21,380 & 29,590 & 19,200 & 9,394 & 27,590 \\
\hline MAX & 16,200 & 16,600 & 12,600 & 17,500 & 17,000 & 49,100 & 41,600 & 24,000 & 36,000 & 32,300 & 21,700 & 29,900 \\
\hline MIN & 11,600 & 8,500 & 5,000 & 4,500 & 11,500 & 11,000 & 16,200 & 19,400 & 18,600 & 11,200 & 1,230 & 22,500 \\
\hline AC-FT & 861,800 & 627,500 & 633,000 & 761,900 & 764,200 & $1,080,000$ & $1,382,000$ & $1,315,000$ & $1,761,000$ & $1,181,000$ & 577,600 & $1,642,000$ \\
\hline
\end{tabular}


Table 15. Daily discharge for the Missouri River at Pierre (station 06440000) for water years 1934-65—Continued

[---, no data; MAX, maximum value; MIN, minimum value; AC-FT, acre-feet]

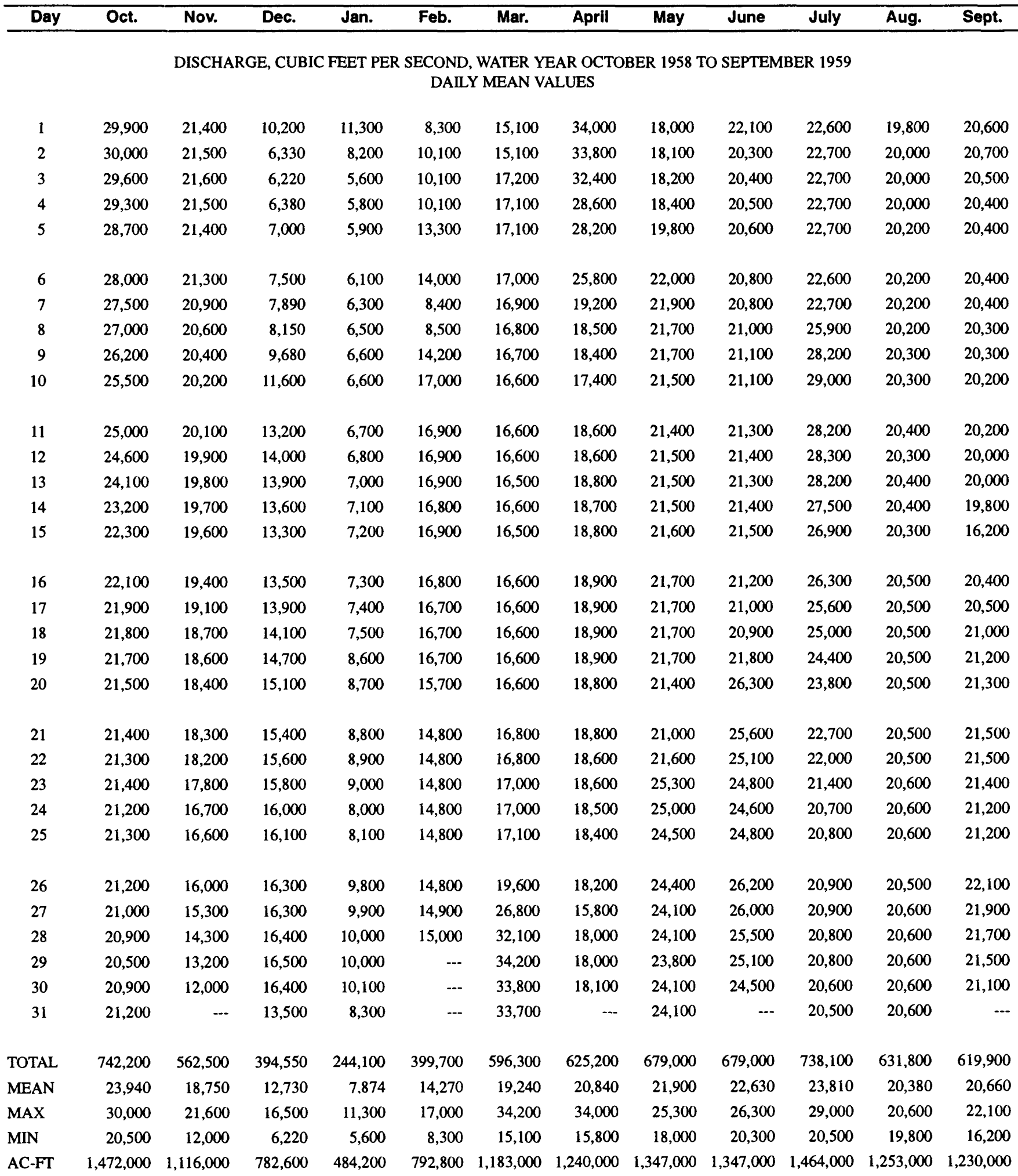


Table 15. Daily discharge for the Missouri River at Pierre (station 06440000) for water years 1934-65-Continued [---, no data; MAX, maximum value; MIN, minimum value; AC-FT, acre-feet]

\begin{tabular}{|c|c|c|c|c|c|c|c|c|c|c|c|c|}
\hline Day & Oct. & Nov. & Dec. & Jan. & Feb. & Mar. & April & May & June & July & Aug. & Sept. \\
\hline \multicolumn{13}{|c|}{$\begin{array}{c}\text { DISCHARGE, CUBIC FEET PER SECOND, WATER YEAR OCTOBER } 1959 \text { TO SEPTEMBER } 1960 \\
\text { DAILY MEAN VALUES }\end{array}$} \\
\hline 1 & 21,000 & 14,400 & 18,200 & 15,100 & 6,500 & 5,800 & 5,400 & 4,720 & 4,800 & 17,600 & 29,900 & 16,300 \\
\hline 2 & 20,900 & 14,100 & 18,100 & 14,900 & 6,500 & 5,500 & 5,400 & 4,770 & 4,800 & 17,500 & 29,900 & 19,500 \\
\hline 3 & 20,800 & 13,800 & 17,900 & 14,500 & 6,500 & 5,100 & 5,500 & 4,720 & 4,770 & 17,400 & 29,900 & 27,900 \\
\hline 4 & 20,800 & 13,100 & 17,600 & 13,900 & 6,400 & 5,200 & 5,500 & 4,720 & 4,830 & 17,300 & 29,900 & 28,100 \\
\hline 5 & 20,700 & 12,100 & 17,300 & 12,900 & 6,100 & 5,200 & 5,400 & 4,750 & 4,860 & 17,300 & 29,800 & 28,000 \\
\hline 6 & 20,800 & 11,700 & 17,300 & 11,500 & 5,300 & 5,300 & 5,700 & 4,690 & 4,940 & 18,200 & 29,900 & 27,900 \\
\hline 7 & 20,900 & 8,700 & 17,300 & 13,000 & 5,400 & 5,300 & 5,700 & 4,690 & 5,600 & 25,200 & 29,800 & 27,900 \\
\hline 8 & 21,000 & 8,490 & 14,500 & 14,000 & 5,500 & 5,100 & 5,700 & 4,720 & 12,000 & 27,700 & 27,800 & 28,000 \\
\hline 9 & 20,800 & 11,100 & 16,400 & 12,000 & 5,900 & 4,900 & 5,000 & 4,660 & 21,000 & 27,800 & 29,400 & 27,200 \\
\hline 10 & 21,000 & 13,900 & 18,300 & 10,000 & 5,500 & 5,000 & 4,270 & 4,660 & 25,800 & 27,900 & 23,000 & 25,400 \\
\hline 11 & 20,900 & 14,600 & 18,500 & 9,700 & 4,500 & 4,900 & 1,630 & 4,640 & 19,600 & 28,100 & 22,600 & 25,300 \\
\hline 12 & 20,800 & 15,200 & 18,500 & 10,000 & 4,800 & 4,800 & 1,560 & 4,660 & 15,700 & 27,800 & 28,600 & 26,700 \\
\hline 13 & 20,400 & 15,400 & 18,500 & 11,300 & 5,100 & 4,700 & 1,360 & 4,720 & 14,900 & 25,600 & 28,700 & 28,900 \\
\hline 14 & 19,900 & 15,000 & 18,400 & 13,500 & 5,400 & 4,700 & 2,140 & 4,610 & 11,800 & 25,700 & 28,400 & 18,700 \\
\hline 15 & 19,400 & 14,100 & 18,300 & 14,500 & 5,600 & 4,700 & 1,490 & 4,640 & 15,600 & 26,100 & 28,600 & 13,500 \\
\hline 16 & 18,800 & 12,500 & 18,100 & 14,700 & 5,800 & 4,700 & 1,740 & 4,660 & 15,500 & 27,600 & 29,600 & 9,490 \\
\hline 17 & 18,300 & 10,300 & 17,900 & 14,700 & 6,100 & 4,700 & 1,700 & 4,690 & 15,500 & 27,700 & 29,300 & 8,490 \\
\hline 18 & 17,900 & 9,060 & 17,700 & 14,600 & 6,400 & 4,700 & 1,750 & 4,770 & 15,500 & 27,700 & 29,200 & 8,320 \\
\hline 19 & 17,600 & 7,000 & 17,600 & 14,500 & 5,800 & 4,800 & 1,780 & 4,800 & 15,500 & 27,600 & 29,000 & 13,000 \\
\hline 20 & 17,300 & 5,580 & 17,500 & 14,600 & 5,400 & 4,800 & 1,750 & 4,750 & 15,500 & 27,400 & 28,900 & 25,600 \\
\hline 21 & 17,100 & 5,540 & 17,600 & 14,600 & 5,300 & 5,600 & 1,770 & 4,750 & 14,000 & 27,100 & 28,700 & 28,700 \\
\hline 22 & 16,900 & 6,350 & 17,300 & 14,700 & 5,400 & 6,500 & 1,850 & 4,800 & 13,500 & 27,000 & 28,900 & 29,000 \\
\hline 23 & 16,700 & 7,650 & 17,300 & 14,800 & 5,600 & 5,500 & 1,850 & 4,830 & 16,800 & 27,100 & 29,200 & 29,300 \\
\hline 24 & 16,300 & 7,790 & 17,000 & 14,800 & 5,800 & 5,300 & 1,890 & 4,750 & 19,600 & 26,900 & 29,200 & 29,900 \\
\hline 25 & 16,100 & 8,100 & 16,700 & 12,400 & 5,800 & 5,200 & 1,930 & 4,660 & 22,500 & 26,700 & 27,000 & 29,700 \\
\hline 26 & 16,000 & 8,630 & 16,100 & 6,300 & 5,600 & 5,100 & 1,870 & 4,940 & 22,300 & 26,800 & 24,500 & 29,600 \\
\hline 27 & 15,800 & 9,890 & 15,900 & 5,900 & 5,300 & 5,200 & 1,910 & 4,860 & 22,200 & 27,400 & 25,900 & 29,400 \\
\hline 28 & 15,700 & 13,200 & 15,600 & 6,000 & 5,500 & 5,300 & 1,960 & 8,770 & 22,100 & 29,400 & 25,600 & 29,600 \\
\hline 29 & 15,600 & 16,700 & 15,600 & 6,200 & 5,700 & 5,300 & 2,790 & 15,800 & 21,100 & 29,400 & 21,900 & 29,200 \\
\hline 30 & 15,100 & 18,500 & 15,500 & 6,400 & -- & 5,300 & 4,690 & 15,300 & 17,700 & 29,800 & 16,300 & 29,000 \\
\hline 31 & 14,600 & --- & 15,500 & 6,600 & $\cdots$ & 5,400 & -- & 5,390 & --- & 29,900 & 16,300 & --- \\
\hline TOTAL & 575,900 & 342,480 & 534,000 & 372,600 & 164,500 & 159,600 & 92,980 & 172,890 & 440,300 & 792,700 & 845,700 & 727,600 \\
\hline MEAN & 18,580 & 11,420 & 17,230 & 12,020 & 5,672 & 5,148 & 3,099 & 5,577 & 14,680 & 25,570 & 27,280 & 24,250 \\
\hline MAX & 21,000 & 18,500 & 18,500 & 15,100 & 6,500 & 6,500 & 5,700 & 15,800 & 25,800 & 29,900 & 29,900 & 29,900 \\
\hline MIN & 14,600 & 5,540 & 14,500 & 5,900 & 4,500 & 4,700 & 1,360 & 4,610 & 4,770 & 17,300 & 16,300 & 8,320 \\
\hline AC-FT & $1,142,000$ & 679,300 & $1,059,000$ & 739,100 & 326,300 & 316,600 & 184,400 & 342,900 & 873,300 & $1,572,000$ & $1,677,000$ & $1,443,000$ \\
\hline
\end{tabular}


Table 15. Daily discharge for the Missouri River at Pierre (station 06440000) for water years 1934-65-Continued [---, no data; MAX, maximum value; MIN, minimum value; AC-FT, acre-feet]

\begin{tabular}{|c|c|c|c|c|c|c|c|c|c|c|c|c|}
\hline Day & Oct. & Nov. & Dec. & Jan. & Feb. & Mar. & April & May & June & July & Aug. & Sept. \\
\hline \multicolumn{13}{|c|}{$\begin{array}{c}\text { DISCHARGE, CUBIC FEET PER SECOND, WATER YEAR OCTOBER } 1960 \text { TO SEPTEMBER } 1961 \\
\text { DAILY MEAN VALUES }\end{array}$} \\
\hline 1 & 29,500 & 8,840 & 12,500 & 12,800 & 6,000 & 4,900 & 15,000 & 28,200 & 18,600 & 26,800 & 29,800 & 27,400 \\
\hline 2 & 29,100 & 8,730 & 17,500 & 13,200 & 6,800 & 4,770 & 17,300 & 30,200 & 18,800 & 26,400 & 29,900 & 26,500 \\
\hline 3 & 28,700 & 9,900 & 18,500 & 12,700 & 7,600 & 4,640 & 19,500 & 30,300 & 19,100 & 26,200 & 29,000 & 26,600 \\
\hline 4 & 28,600 & 11,300 & 17,500 & 9,800 & 8,200 & 4,740 & 19,800 & 30,600 & 19,400 & 26,400 & 29,800 & 26,600 \\
\hline 5 & 28,700 & 11,300 & 16,500 & 7,400 & 8,400 & 4,800 & 21,700 & 30,600 & 19,600 & 27,200 & 29,800 & 25,600 \\
\hline 6 & 28,800 & 11,200 & 15,000 & 7,600 & 8,300 & 4,900 & 22,500 & 30,700 & 19,400 & 28,600 & 29,400 & 24,300 \\
\hline 7 & 28,900 & 10,800 & 14,000 & 7,600 & 8,200 & 4,900 & 25,000 & 30,800 & 19,200 & 28,800 & 29,200 & 22,500 \\
\hline 8 & 28,800 & 11,300 & 13,500 & 8,200 & 8,100 & 4,880 & 25,000 & 30,700 & 20,600 & 29,000 & 29,300 & 20,600 \\
\hline 9 & 29,400 & 11,000 & 13,200 & 7,800 & 8,200 & 4,930 & 25,000 & 30,500 & 22,900 & 28,900 & 29,300 & 18,800 \\
\hline 10 & 28,800 & 11,000 & 13,400 & 7,400 & 8,000 & 4,930 & 24,900 & 30,400 & 23,400 & 28,800 & 27,300 & 17,900 \\
\hline 11 & 27,500 & 10,900 & 13,500 & 6,200 & 7,400 & 4,930 & 24,900 & 30,200 & 23,300 & 28,700 & 25,000 & 16,800 \\
\hline 12 & 27,200 & 10,900 & 13,700 & 5,400 & 7,500 & 4,900 & 25,900 & 30,100 & 23,700 & 28,700 & 24,500 & 16,200 \\
\hline 13 & 27,400 & 10,800 & 13,700 & 5,600 & 7,300 & 4,510 & 29,400 & 30,300 & 25,300 & 29,000 & 24,500 & 15,500 \\
\hline 14 & 27,500 & 10,800 & 13,700 & 6,000 & 7,200 & 4,850 & 29,300 & 30,300 & 22,000 & 28,900 & 24,500 & 14,900 \\
\hline 15 & 26,200 & 11,000 & 13,600 & 6,000 & 6,600 & 4,960 & 29,300 & 26,600 & 13,400 & 29,200 & 26,000 & 14,400 \\
\hline 16 & 26,400 & 11,100 & 13,700 & 6,000 & 5,600 & 4,990 & 29,400 & 28,300 & 6,410 & 28,800 & 25,300 & 11,800 \\
\hline 17 & 26,300 & 8,770 & 13,700 & 5,800 & 5,600 & 5,100 & 29,300 & 27,900 & 8,580 & 29,200 & 26,500 & 9,510 \\
\hline 18 & 26,000 & 4,960 & 13,800 & 5,500 & 5,600 & 5,210 & 29,400 & 23,900 & 5,460 & 30,200 & 25,700 & 9,690 \\
\hline 19 & 24,000 & 4,510 & 14,100 & 5,200 & 5,700 & 5,240 & 29,500 & 23,200 & 5,070 & 30,200 & 24,400 & 9,830 \\
\hline 20 & 21,900 & 4,720 & 14,300 & 5,100 & 5,240 & 7,390 & 29,500 & 22,800 & 6,940 & 30,000 & 24,200 & 9,900 \\
\hline 21 & 20,200 & 5,040 & 14,400 & 5,100 & 4,720 & 11,000 & 29,300 & 22,700 & 11,700 & 30,200 & 24,400 & 9,900 \\
\hline 22 & 18,700 & 5,000 & 14,500 & 5,200 & 4,770 & 11,000 & 29,000 & 20,900 & 17,200 & 30,400 & 24,700 & 9,690 \\
\hline 23 & 17,300 & 5,200 & 14,600 & 5,000 & 4,770 & 11,000 & 29,000 & 17,900 & 22,800 & 30,100 & 25,400 & 8,380 \\
\hline 24 & 16,100 & 5,400 & 14,600 & 4,900 & 4,900 & 13,200 & 27,600 & 17,700 & 22,900 & 30,200 & 25,200 & 8,450 \\
\hline 25 & 15,200 & 5,600 & 14,500 & 4,900 & 4,960 & 17,100 & 22,700 & 17,500 & 22,900 & 30,000 & 26,000 & 8,480 \\
\hline 26 & 14,500 & 5,800 & 14,500 & 5,300 & 4,900 & 16,900 & 22,500 & 16,800 & 23,400 & 26,600 & 27,500 & 8,480 \\
\hline 27 & 14,000 & 6,000 & 14,400 & 5,600 & 4,930 & 16,800 & 22,400 & 16,800 & 24,800 & 21,600 & 27,100 & 8,420 \\
\hline 28 & 13,400 & 6,200 & 14,000 & 5,500 & 4,930 & 14,100 & 23,800 & 16,600 & 24,600 & 19,200 & 27,000 & 9,290 \\
\hline 29 & 12,900 & 6,400 & 13,800 & 5,600 & --- & 10,900 & 26,100 & 16,900 & 25,000 & 15,800 & 27,200 & 8,310 \\
\hline 30 & 12,400 & 7,600 & 13,300 & 5,600 & --. & 11,000 & 26,000 & 18,200 & 26,900 & 15,600 & 26,800 & 8,200 \\
\hline 31 & 11,400 & --- & 12,700 & 5,800 & -- & 11,600 & $\cdots$ & 18,300 & -- & 18,900 & 27,000 & $\cdots$ \\
\hline TOTAL & 715,800 & 252,070 & 444,700 & 209,800 & 180,420 & 245,070 & 760,000 & 776,900 & 563,360 & 838,600 & 831,700 & 452,930 \\
\hline MEAN & 23,090 & 8,402 & 14,350 & 6,768 & 6,444 & 7,905 & 25,330 & 25,060 & 18,780 & 27,050 & 26,830 & 15,100 \\
\hline MAX & 29,500 & 11,300 & 18,500 & 13,200 & 8,400 & 17,100 & 29,500 & 30,800 & 26,900 & 30,400 & 29,900 & 27,400 \\
\hline MIN & 11,400 & 4,510 & 12,500 & 4,900 & 4,720 & 4,510 & 15,000 & 16,600 & 5,070 & 15,600 & 24,200 & 8,200 \\
\hline AC-FT & $1,420,000$ & 500,000 & 882,100 & 416,100 & 357,900 & 486,100 & $1,507,000$ & $1,541,000$ & $1,117,000$ & $1,663,000$ & $1,650,000$ & 898,400 \\
\hline
\end{tabular}


Table 15. Daily discharge for the Missouri River at Pierre (station 06440000) for water years 1934-65-Continued [---, no data; MAX, maximum value; MIN, minimum value; AC-FT, acre-feet]

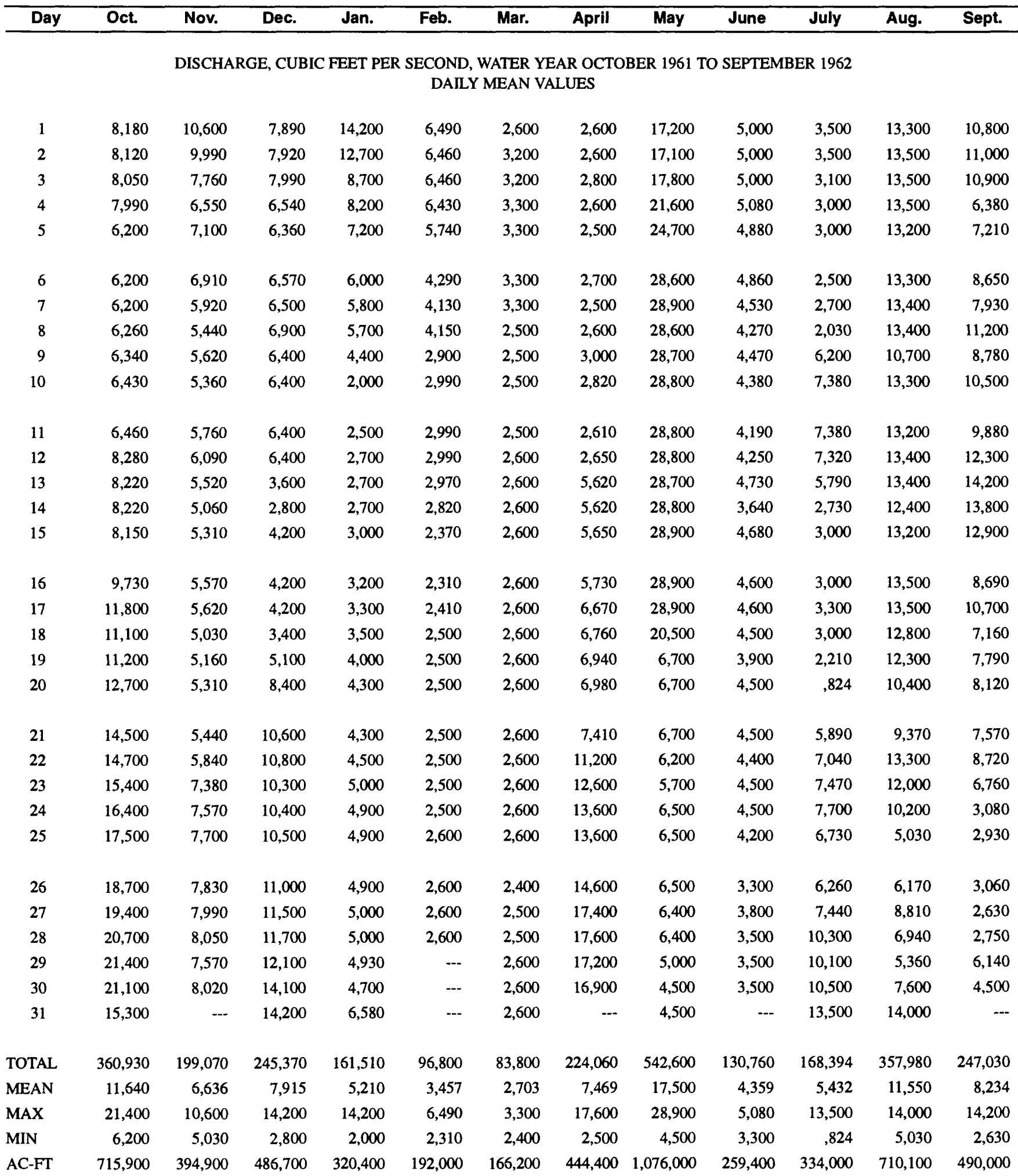


Table 15. Daily discharge for the Missoúri River at Pierre (station 06440000) for water years 1934-65—Continued [---, no data; MAX, maximum value; MIN, minimum value; AC-FT, acre-feet]

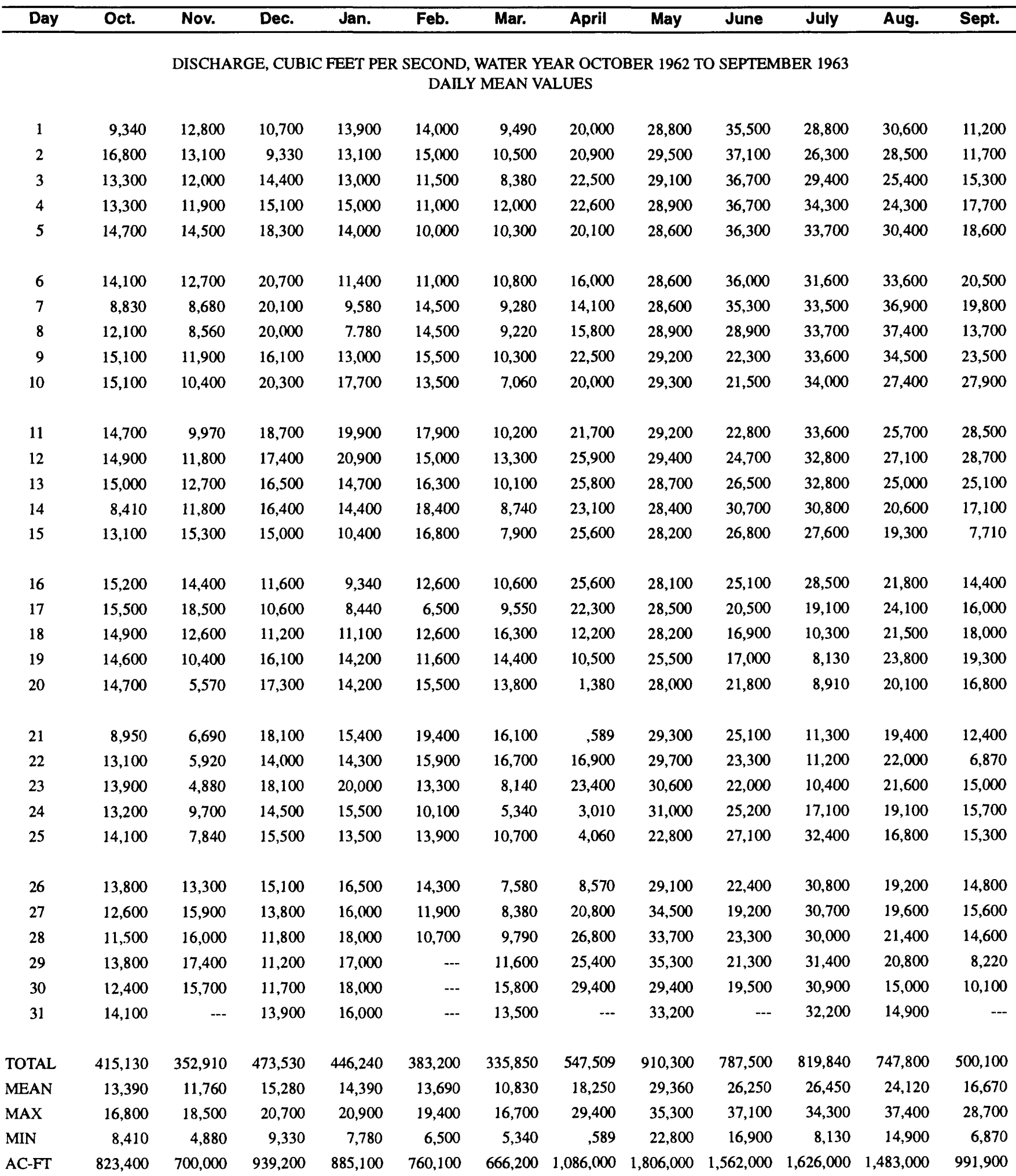


Table 15. Daily discharge for the Missouri River at Pierre (station 06440000) for water years 1934-65-Continued

[---, no data; MAX, maximum value; MIN, minimum value; AC-FT, acre-feet]

\begin{tabular}{|c|c|c|c|c|c|c|c|c|c|c|c|c|}
\hline Day & Oct. & Nov. & Dec. & Jan. & Feb. & Mar. & April & May & June & July & Aug. & Sept. \\
\hline \multicolumn{13}{|c|}{$\begin{array}{c}\text { DISCHARGE, CUBIC FEET PER SECOND, WATER YEAR OCTOBER } 1963 \text { TO SEPTEMBER } 1964 \\
\text { DAILY MEAN VALUES }\end{array}$} \\
\hline 1 & 10,600 & 16,700 & 16,500 & 18,400 & 16,300 & 9,290 & 20,000 & 21,400 & 20,400 & 22,500 & 35,000 & 38,300 \\
\hline 2 & 24,300 & 17,400 & 21,100 & 19,700 & 12,100 & 16,800 & 19,900 & 20,900 & 21,500 & 25,000 & 29,100 & 42,000 \\
\hline 3 & 11,800 & 10,400 & 19,400 & 23,000 & 16,600 & 23,400 & 22,800 & 17,000 & 19,400 & 27,900 & 35,600 & 41,500 \\
\hline 4 & 10,000 & 16,400 & 17,600 & 20,600 & 17,800 & 22,900 & 33,200 & 8,650 & 18,400 & 18,800 & 37,000 & 22,500 \\
\hline 5 & 9,560 & 18,500 & 17,000 & 17,600 & 17,400 & 23,900 & 30,300 & 6,950 & 29,400 & 18,000 & 37,900 & 15,600 \\
\hline u & 6,760 & 17,800 & 16,700 & 22,700 & 18,500 & 28,800 & 31,700 & 6,310 & 27,100 & 26,600 & 33,000 & 12,600 \\
\hline \multirow[t]{4}{*}{7} & 15,100 & 19,300 & 16,300 & 21,600 & 18,400 & 30,200 & 27,600 & 7,420 & 33,000 & 28,900 & 28,200 & 16,500 \\
\hline & 17,000 & 19,800 & 15,900 & 23,100 & 18,300 & 21,400 & 21,400 & 8,950 & 33,200 & 28,800 & 24,200 & 24,500 \\
\hline & 17,800 & 14,300 & 21,200 & 27,700 & 14,100 & 24,000 & 20,200 & 6,820 & 36,200 & 29,900 & 18,700 & 23,700 \\
\hline & 16,100 & 10,200 & 23,200 & 30,300 & 19,200 & 22,100 & 16,400 & 3,000 & 33,900 & 25,600 & 28,400 & 20,000 \\
\hline 11 & 16,400 & 14,200 & 23,400 & 30,800 & 21,800 & 21,400 & 14,400 & 5,000 & 30,100 & 19,800 & 27,700 & 14,400 \\
\hline 12 & 14,900 & 17,200 & 25,400 & 27,600 & 19,600 & 21,200 & 7,320 & 6,500 & 31,200 & 19,100 & 26,800 & 10,000 \\
\hline 13 & 6,220 & 16,300 & 26,400 & 30,100 & 16,600 & 20,700 & 16,100 & 8,000 & 23,500 & 24,400 & 29,100 & 6,000 \\
\hline 14 & 14,400 & 16,300 & 26,100 & 27,400 & 16,400 & 20,600 & 23,400 & 10,400 & 27,400 & 25,600 & 32,000 & 8,000 \\
\hline 15 & 17,200 & 14,900 & 24,900 & 23,100 & 18,300 & 14,200 & 21,400 & 13,100 & 24,700 & 28,000 & 29,800 & 10,000 \\
\hline 16 & 18,700 & 13,700 & 27,300 & 21,500 & 15,400 & 20,900 & 16,300 & 17,300 & 26,000 & 32,600 & 24,700 & 8,000 \\
\hline 17 & 18,700 & 7,740 & 27,300 & 20,200 & 17,600 & 23,200 & 16,300 & 18,600 & 24,500 & 34,800 & 29,700 & 6,500 \\
\hline 18 & 22,400 & 13,300 & 25,800 & 19,900 & 17,300 & 25,500 & 19,500 & 18,700 & 28,100 & 34,200 & 33,800 & 7,000 \\
\hline 19 & 25,000 & 11,300 & 26,800 & 17,500 & 18,500 & 25,000 & 3,000 & 17,700 & 32,000 & 29,900 & 35,400 & 8,500 \\
\hline 20 & 17,800 & 14,000 & 29,600 & 18,400 & 18,600 & 26,200 & 16,000 & 20,000 & 15,600 & 35,200 & 34,800 & 4,000 \\
\hline 21 & 23,500 & 13,700 & 28,000 & 17,200 & 19,200 & 3,610 & 23,000 & 23,200 & 6,000 & 37,600 & 31,500 & 6,000 \\
\hline 22 & 23,000 & 14,800 & 21,000 & 17,400 & 19,100 & 1,400 & 21,200 & 24,500 & 10,000 & 37,500 & 31,100 & 8,000 \\
\hline 23 & 21,500 & 11,400 & 28,500 & 20,900 & 18,900 & 13,000 & 21,600 & 21,600 & 13,600 & 36,800 & 23,400 & 9,500 \\
\hline 24 & 20,200 & 13,100 & 19,900 & 21,500 & 19,900 & 25,200 & 20,400 & 18,100 & 12,900 & 35,400 & 30,900 & 9,500 \\
\hline 25 & 21,400 & 14,000 & 13,900 & 19,600 & 21,200 & 26,400 & 19,400 & 22,800 & 14,200 & 24,800 & 34,200 & 7,500 \\
\hline 26 & 16,400 & 15,200 & 18,000 & 17,100 & 20,900 & 26,700 & 17,300 & 21,600 & 17,900 & 19,800 & 34,000 & 5,000 \\
\hline 27 & 3,610 & 16,100 & 22,400 & 19,800 & 20,400 & 20,800 & 21,000 & 22,300 & 13,100 & 30,500 & 34,400 & 2,500 \\
\hline 28 & 18,000 & 15,200 & 23,400 & 22,200 & 17,600 & 21,400 & 19,000 & 24,400 & 9,150 & 30,500 & 32,200 & 5,000 \\
\hline 29 & 22,500 & 17,900 & 22,500 & 17,800 & 14,600 & 17,800 & 17,700 & 20,400 & 16,100 & 22,400 & 28,500 & 7,000 \\
\hline 30 & 21,300 & 18,500 & 26,700 & 17,300 & --. & 20,100 & 16,700 & 15,300 & 17,800 & 20,900 & 20,700 & 11,500 \\
\hline 31 & 19,200 & --- & 25,900 & 17,300 & --- & 20,000 & --- & 10,800 & -.. & 29,400 & 31,300 & -.. \\
\hline TOTAL & 521,350 & 449,640 & 698,100 & 669,300 & 520,600 & 638,100 & 594,520 & 467,700 & 666,350 & 861,200 & 943,100 & 411,100 \\
\hline MEAN & 16,820 & 14,990 & 22,520 & 21,590 & 17,950 & 20,580 & 19,820 & 15,090 & 22,210 & 27,780 & 30,420 & 13,700 \\
\hline MAX & 25,000 & 19,800 & 29,600 & 30,800 & 21,800 & 30,200 & 33,200 & 24,500 & 36,200 & 37,600 & 37,900 & 42,000 \\
\hline MIN & 3,610 & 7,740 & 13,900 & 17,100 & 12,100 & 1,400 & 3,000 & 3,000 & 6,000 & 18,000 & 18,700 & 2,500 \\
\hline AC-FT & $1,034,000$ & 891,900 & $1,385,000$ & $1,328,000$ & $1,033,000$ & $1,266,000$ & $1,179,000$ & 927,700 & $1,322,000$ & $1,708,000$ & $1,871,000$ & 815,400 \\
\hline
\end{tabular}


Table 15. Daily discharge for the Missouri River at Pierre (station 06440000) for water years 1934-65-Continued [--, no data; MAX, maximum value; MIN, minimum value; AC-FT, acre-feet $]$

\begin{tabular}{|c|c|c|c|c|c|c|c|c|c|c|c|c|}
\hline Day & Oct. & Nov. & Dec. & Jan. & Feb. & Mar. & April & May & June & July & Aug. & Sept. \\
\hline \multicolumn{13}{|c|}{$\begin{array}{c}\text { DISCHARGE, CUBIC FEET PER SECOND, WATER YEAR OCTOBER } 1964 \text { TO SEPTEMBER } 1965 \\
\text { DAILY MEAN VALUES }\end{array}$} \\
\hline 1 & 14,300 & 11,300 & 27,900 & 14,900 & 19,900 & 17,200 & 14,200 & 25,200 & 16,000 & 13,500 & 24,200 & 23,700 \\
\hline 2 & 13,000 & 12,100 & 29,600 & 16,400 & 21,500 & 17,800 & 18,600 & 18,000 & 17,300 & 20,500 & 24,300 & 23,800 \\
\hline 3 & 7,380 & 11,900 & 31,200 & 16,000 & 21,800 & 18,400 & 22,600 & 21,600 & 19,100 & 20,000 & 26,300 & 26,400 \\
\hline 4 & 6,760 & 12,300 & 28,700 & 16,800 & 19,800 & 17,300 & 17,100 & 22,400 & 23,300 & 16,300 & 24,500 & 27,300 \\
\hline 5 & 11,100 & 14,500 & 26,500 & 16,100 & 16,200 & 15,600 & 22,800 & 23,200 & 22,100 & 16,700 & 24,800 & 21,100 \\
\hline 6 & 11,600 & 13,600 & 22,200 & 18,200 & 14,500 & 15,700 & 20,000 & 22,500 & 16,800 & 18,600 & 25,200 & 22,400 \\
\hline 7 & 14,600 & 12,000 & 23,200 & 16,900 & 12,700 & 11,800 & 16,000 & 23,000 & 18,200 & 20,000 & 22,700 & 29,000 \\
\hline 8 & 12,500 & 6,950 & 20,300 & 19,700 & 16,800 & 16,200 & 12,900 & 21,800 & 15,600 & 17,500 & 21,600 & 32,000 \\
\hline 9 & 12,900 & 17,100 & 18,300 & 21,800 & 14,700 & 16,300 & 18,300 & 15,400 & 15,200 & 16,500 & 26,600 & 33,000 \\
\hline 10 & 8,920 & 22,700 & 19,300 & 18,200 & 16,600 & 16,300 & 13,900 & 19,600 & 14,800 & 14,500 & 30,800 & 30,100 \\
\hline 11 & 5,000 & 19,700 & 18,700 & 20,800 & 18,500 & 15,100 & 8,490 & 21,900 & 16,900 & 16,900 & 33,400 & 30,100 \\
\hline 12 & 8,880 & 20,200 & 15,400 & 18,300 & 17,100 & 15,100 & 10,300 & 23,800 & 17,500 & 19,800 & 37,400 & 23,900 \\
\hline 13 & 9,000 & 26,100 & 16,400 & 20,600 & 17,100 & 13,300 & 13,500 & 24,500 & 12,900 & 21,200 & 39,000 & 27,300 \\
\hline 14 & 14,000 & 20,700 & 23,200 & 20,200 & 11,400 & 9,220 & 14,000 & 22,900 & 21,000 & 19,500 & 33,200 & 30,100 \\
\hline 15 & 18,000 & 14,600 & 22,900 & 19,100 & 15,600 & 14,300 & 16,400 & 18,900 & 21,600 & 23,000 & 24,900 & 26,900 \\
\hline 16 & 21,000 & 25,000 & 22,400 & 19,700 & 14,900 & 15,300 & 15,300 & 11,500 & 19,500 & 25,700 & 30,400 & 28,600 \\
\hline 17 & 20,000 & 33,900 & 26,600 & 14,400 & 13,400 & 18,000 & 13,900 & 23,200 & 20,100 & 26,300 & 32,100 & 26,200 \\
\hline 18 & 15,000 & 25,800 & 23,900 & 17,400 & 15,700 & 19,100 & 10,500 & 20,200 & 19,300 & 25,600 & 28,800 & 27,600 \\
\hline 19 & 19,400 & 26,700 & 18,200 & 16,500 & 14,500 & 18,600 & 12,600 & 18,300 & 17,300 & 26,300 & 29,800 & 24,500 \\
\hline 20 & 15,500 & 28,800 & 17,500 & 16,600 & 12,200 & 18,300 & 11,300 & 20,300 & 12,400 & 25,800 & 27,600 & 26,600 \\
\hline 21 & 10,500 & 21,600 & 20,000 & 14,900 & 14,100 & 13,100 & 12,400 & 16,900 & 19,600 & 29,100 & 24,700 & 27,300 \\
\hline 22 & 11,300 & 9,150 & 18,800 & 16,600 & 22,500 & 16,400 & 15,000 & 18,200 & 27,300 & 31,100 & 20,100 & 25,800 \\
\hline 23 & 11,600 & 12,800 & 22,000 & 18,000 & 22,500 & 16,000 & 16,500 & 15,000 & 26,100 & 28,900 & 27,400 & 25,500 \\
\hline 24 & 9,120 & 16,100 & 20,900 & 11,400 & 21,000 & 15,200 & 18,100 & 19,700 & 25,600 & 26,800 & 29,400 & 22,800 \\
\hline 25 & 8,090 & 9,730 & 16,800 & 17,500 & 16,600 & 13,900 & 14,400 & 18,500 & 25,900 & 24,900 & 31,200 & 22,800 \\
\hline 26 & 10,000 & 13,200 & 19,600 & 17,600 & 15,700 & 13,200 & 18,400 & 20,700 & 17,600 & 27,400 & 31,300 & 22,400 \\
\hline 27 & 11,100 & 18,900 & 19,800 & 19,200 & 12,200 & 11,500 & 18,200 & 19,800 & 10,600 & 29,500 & 29,500 & 26,500 \\
\hline 28 & 16,800 & 28,500 & 21,400 & 23,600 & 8,980 & 9,530 & 19,900 & 17,900 & 10,800 & 28,600 & 28,400 & 25,600 \\
\hline 29 & 17,500 & 29,800 & 20,800 & 22,300 & -- & 12,900 & 20,000 & 14,700 & 7,900 & 29,700 & 22,100 & 27,400 \\
\hline 30 & 16,400 & 32,300 & 19,200 & 18,200 & -- & 13,000 & 23,200 & 11,300 & 6,890 & 28,700 & 26,300 & 15,900 \\
\hline 31 & 16,500 & --- & 18,600 & 15,100 & --- & 11,100 & -.. & 9,700 & -. & 24,700 & 25,400 & -- \\
\hline TOTAL & 397,750 & 568,030 & 670,300 & 553,000 & 458,480 & 464,750 & 478,790 & 600,600 & 535,190 & 713,600 & 863,400 & 782,600 \\
\hline MEAN & 12,830 & 18,930 & 21,620 & 17,840 & 16,370 & 14,990 & 15,960 & 19,370 & 17,840 & 23,020 & 27,850 & 26,090 \\
\hline MAX & 21,000 & 33,900 & 31,200 & 23,600 & 22,500 & 19,100 & 23,200 & 25,200 & 27,300 & 31,100 & 39,000 & 33,000 \\
\hline MIN & 5,000 & 6,950 & 15,400 & 11,400 & 8,980 & 9,220 & 8,490 & 9,700 & 6,890 & 13,500 & 20,100 & 15,900 \\
\hline AC-FT & 788,900 & $1,127,000$ & $1,330,000$ & $1,097,000$ & 909,400 & 921,800 & 949,700 & $1,191,000$ & $1,062,000$ & $1,415,000$ & $1,713,000$ & $1,552,000$ \\
\hline
\end{tabular}


Table 16. Daily discharge for the Missouri River at Chamberlain (station 06443000) for the periods September 1928 to September 1929 and April 1945 to September 1954

[---, no data; MAX, maximum value; MIN, minimum value; AC-FT, acre-feet]

\begin{tabular}{|c|c|c|c|c|c|c|c|c|c|c|c|c|}
\hline Day & Oct. & Nov. & Dec. & Jan. & Feb. & Mar. & April & May & June & July & Aug. & Sept. \\
\hline \multicolumn{13}{|c|}{$\begin{array}{l}\text { DISCHARGE, CUBIC FEET PER SECOND, WATER YEAR OCTOBER } 1927 \text { TO SEPTEMBER } 1928 \\
\text { DAILY MEAN VALUES }\end{array}$} \\
\hline 1 & --- & --- & -- & --. & --- & -- & --- & -.. & $\ldots$ & -.. & --- & 20,300 \\
\hline 2 & $\ldots$ & $\ldots$ & $\ldots$ & $\ldots$ & --- & --. & --- & -.- & ... & --. & --- & 20,100 \\
\hline 3 &.-- & -.. & $\ldots$ & --. & --- & --- & --- & -.. & -.. & -.. & -.- & 17,600 \\
\hline 4 & --. & --- & -.- & -.. & --. & ... & $\ldots$ & -.- & --. & --- & -.. & 18,800 \\
\hline 5 & --. & --- & $\ldots$ & $\ldots$ & -.- & -- & --- & -.- & $\ldots$ & ... & $\ldots$ & 18,500 \\
\hline 6 & --- & --- & $\ldots$ & ... & --. & --. & ... & ... & $\ldots$ & ... & $\ldots$ & 18,900 \\
\hline 7 & --- & --- & -.- & $\ldots$ & -.. & -.- & --- & -.. & -.. & -.. & $\ldots$ & 18,600 \\
\hline 8 & --- & -.- & --- & --- &.-- & --. & --- & $\ldots$ & $\ldots$ & $\cdots$ & $\ldots$ & 18,100 \\
\hline 9 & ... & ... & ... & ... & --- & ... & ... & ... & ... & --- & ... & 18,200 \\
\hline 10 & --- & --- & --- & ... & -.. & -.- & --- & -.. & -.- & --. & -.. & 18,900 \\
\hline 11 & -.- & $\ldots$ & ... & --- & --- & -.. & -.. & --- & --- & --- & --- & 19,900 \\
\hline 12 & ..- & ... & ... & -.- & ... & ... & ... & $\ldots$ & $\ldots$ & $\ldots$ & $\ldots$ & 20,200 \\
\hline 13 & -.. & -.. & -.- & $\ldots$ & $\ldots$ & -- & -.. & --. & -.. & --- & -.. & 20,600 \\
\hline 14 & --- & --- & --- & --. & --- & --- & --- & --- & $\cdots$ & $\ldots$ & $\cdots$ & 21,000 \\
\hline 15 & $\ldots$ & $\ldots$ & --. & ... & $\ldots$ & -.. & ... & $\cdots$ & -- & --- & --- & 20,400 \\
\hline 16 & $\ldots$ & ... & $\ldots$ & -.. & --- & $\ldots$ & -- & -.- & --- & --. & --- & 20,300 \\
\hline 17 & -.. & ... & --- & -.- & -.. & --. & -.. & -.- & $\ldots$ & -.. & $\ldots$ & 20,000 \\
\hline 18 & ... & ... & ... & ... & ... & ... & -.. & ... & -..- & -..- & -.- & 21,400 \\
\hline 19 & --. & -.- & --- & -.. & -.. & --- & ... & -.. & --- & --- & ... & 27,600 \\
\hline 20 & --- & -- & -.- & -.. & --- & --- & --. & --- & --- & ... & $\ldots$ & 36,700 \\
\hline 21 & -.. & -.. &.-- & --- & --- & --. & --- & --- & --. &.-- & $\ldots$ & 31,400 \\
\hline 22 & --. & .-. & -.- & -.. & -.- & -.. & -.. & -.. & --- & --- & --- & 24,300 \\
\hline 23 & --- & --. & --- & --. & --- & -.- & $\ldots$ & $\cdots$ & --- & ..- & --. & 19,700 \\
\hline 24 & -- & -.- & ... & $\ldots$ & --. & -.. & -.- & -.. & -.. & --. & --- & 18,600 \\
\hline 25 & -- & -.- & $\ldots$ & -.- & -.. & --. & --- & -- & $\ldots$ & -.. & ..- & 17,800 \\
\hline 26 & --- & -.. & -.. & -.. & -. & -.. & -.. & -- & $\cdots$ & $--\cdot$ & -- & 17,200 \\
\hline 27 & --. & -. & -.. & --. & -.- & -.. & --. & --. & $\ldots$ & $\ldots$ & -.. & 16,600 \\
\hline 28 & ..- & -.. & -.. & $\ldots$ & -.. & $\ldots$ & --. & --. & -- & --- & $\ldots$ & 16,100 \\
\hline 29 & --. & --. & --. & -.. & --- & -.. & ... & $\ldots$ & $\ldots$ & -.. & $\ldots$ & 16,200 \\
\hline 30 & ... & $\ldots$ & -.. & -.. & $\ldots$ &.-- & -.- & -.- & --. & --. & -.. & 16,200 \\
\hline 31 & -- & -.. & -.. & -.. & -.- & --- & -- & $\ldots$ & -.. & -.- & $\ldots$ & -- \\
\hline TOTAL & --- & --- & --- & -- & -.. & --- & -- & -- & $\cdots$ & -- & -- & 610,200 \\
\hline MEAN & $\ldots$ & $\ldots$ & ... & $\ldots$ & ... & ... & -.. & -.- & ..- & -.. & ... & 20,340 \\
\hline MAX & --. & $\ldots$ & $\ldots$ & -. & $\ldots$ & $\ldots$ & -.- & -.. & -- & $\ldots$ & --. & 36,700 \\
\hline MIN & $\ldots$ & ... & -.- & ... & -.. & -.. & ... & $\ldots$ & ... & -.- & --- & 16,100 \\
\hline AC-FT & -.. & -- & -.. & -.. & -.. & -.. & ... & $\ldots$ & -.- & $\ldots$ & --- & $1,210,000$ \\
\hline
\end{tabular}


Table 16. Daily discharge for the Missouri River at Chamberlain (station 06443000) for the periods September 1928 to September 1929 and April 1945 to September 1954-Continued

[---, no data; MAX, maximum value; MIN, minimum value; AC-FT, acre-feet $]$

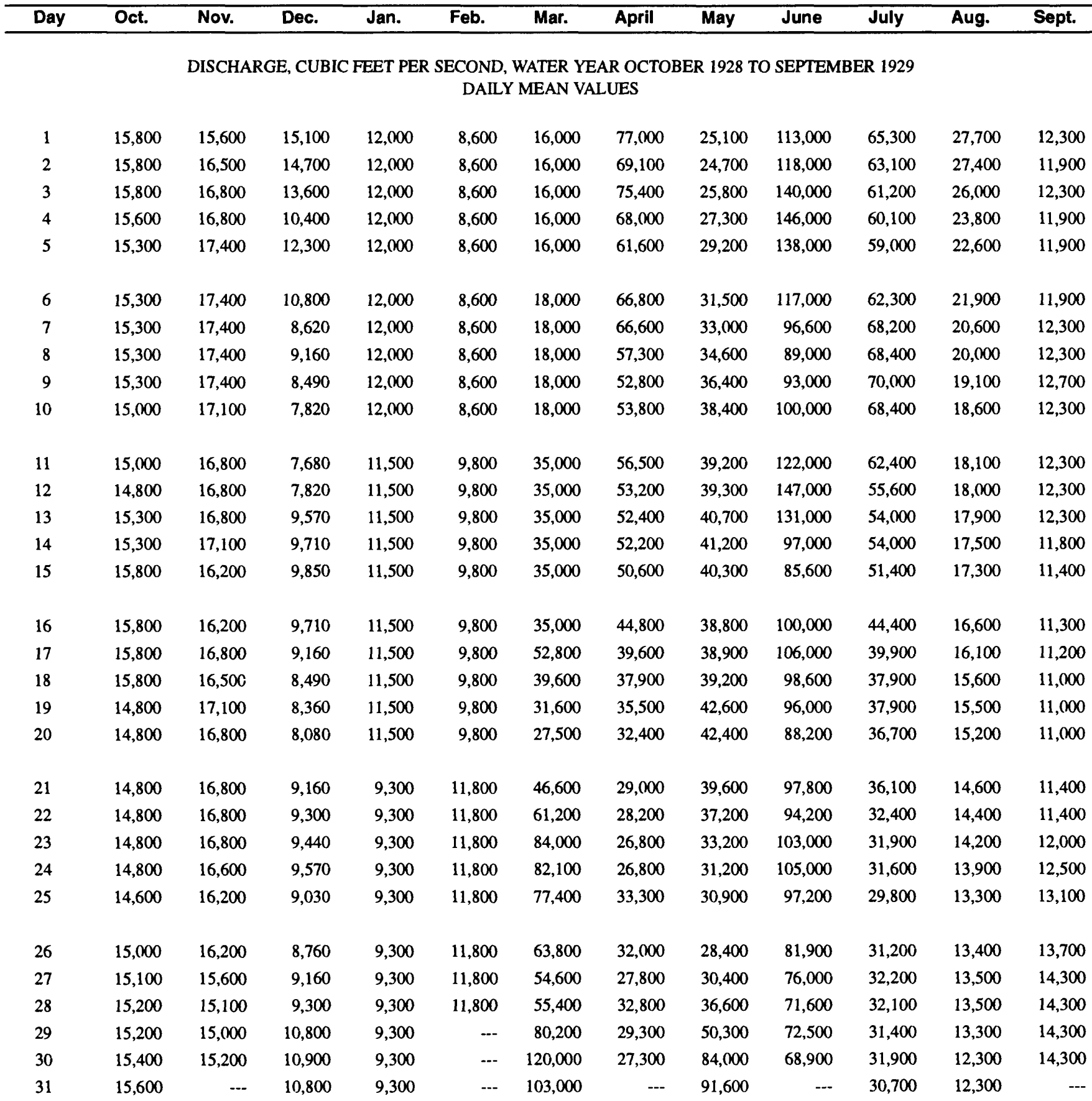

\begin{tabular}{|c|c|c|c|c|c|c|c|c|c|c|c|c|}
\hline TOTAL & 473,000 & 497,200 & 305,640 & 337,300 & 278,400 & $1,359,800$ & $1,396,800$ & $1,202,000$ & $3,090,100$ & $1,471,500$ & 544,200 & 368,700 \\
\hline MEAN & 15,260 & 16,570 & 9,859 & 10,880 & 9,943 & 43,860 & 46,560 & 38,770 & 103,000 & 47,470 & 17,550 & 12,290 \\
\hline MAX & 15,800 & 17,400 & 15,100 & 12,000 & 11,800 & 120,000 & 77,000 & 91,600 & 147,000 & 70,000 & 27,700 & 14,300 \\
\hline MIN & 14,600 & 15,000 & 7,680 & 9,300 & 8,600 & 16,000 & 26,800 & 24,700 & 68,900 & 29,800 & 12,300 & 11,000 \\
\hline AC-FT & 938,200 & 986,200 & 606,200 & 669,000 & 552,200 & $2,697,000$ & $2,771,000$ & $2,384,000$ & $6,129,000$ & $2,919,000$ & $1,079,000$ & 731,300 \\
\hline
\end{tabular}


Table 16. Daily discharge for the Missouri River at Chamberlain (station 06443000) for the periods September 1928 to September 1929 and April 1945 to September 1954-Continued

[---, no data; MAX, maximum value; MIN, minimum value; AC-FT, acre-feet $]$

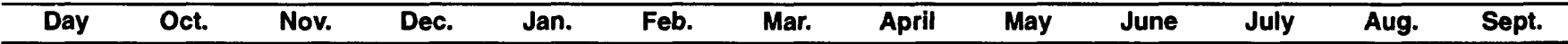

DISCHARGE, CUBIC FEET PER SECOND, WATER YEAR OCTOBER 1944 TO SEPTEMBER 1945 DAILY MEAN VALUES

\begin{tabular}{|c|c|c|c|c|c|c|c|c|c|c|c|c|}
\hline 1 & --- & --- & --- & -.- & -- & -.. & 41,100 & 11,600 & 20,500 & 67,600 & 33,800 & 19,000 \\
\hline 2 & $\ldots$ & --. & -.. & --. & -- & -.. & 35,900 & 11,300 & 20,000 & 78,600 & 32,600 & 18,700 \\
\hline 3 & --- & --- & --- & --- & --- & --- & 34,200 & 11,300 & 20,000 & 77,400 & 31,500 & 18,700 \\
\hline 4 &.-- & --- & --- & --- & -- & --- & 31,800 & 11,100 & 20,500 & 72,900 & 29,200 & 19,200 \\
\hline 5 & -.- & --- & $\cdots$ & --- & --- & --- & 28,800 & 11,100 & 20,800 & 72,900 & 28,200 & 19,700 \\
\hline 6 & --- & --. & --- & --- & --- & $\cdots$ & 27,600 & 11,100 & 21,900 & 62,500 & 26,700 & 20,200 \\
\hline 7 & --- & --- & --- & -.. &.-- & --- & 25,500 & 10,900 & 23,700 & 51,400 & 25,800 & 20,000 \\
\hline 8 & --- & --- & --. & --. & --- & --- & 23,100 & 11,100 & 27,300 & 43,100 & 25,500 & 19,400 \\
\hline 9 & --- & --- & $\ldots$ &.-- & -.. & --- & 21,600 & 11,600 & 50,600 & 39,400 & 24,600 & 19,400 \\
\hline 10 & --- & --- & -- & --. & --- & --- & 20,200 & 12,000 & 43,800 & 37,300 & 23,100 & 19,700 \\
\hline 11 & --- & --- & --- & --- & --- & --- & 20,800 & 13,000 & 49,000 & 36,400 & 21,400 & 19,700 \\
\hline 12 & --- & -.. & --- & -.- & --- & --- & 20,000 & 13,000 & 59,600 & 35,000 & 19,700 & 21,100 \\
\hline 13 & -- & --- & --- & -.. & --- & --- & 18,700 & 12,800 & 62,500 & 33,800 & 19,200 & 22,800 \\
\hline 14 & --- & --- & --. & --- & --- & -.. & 18,000 & 12,600 & 61,500 & 34,200 & 19,400 & 23,700 \\
\hline 15 & --- & --- & --- & --- & --- & ... & 17,600 & 12,500 & 63,500 & 36,400 & 19,700 & 23,400 \\
\hline 16 & --. & -.- & --- & --- & -.- & --- & 17,100 & 12,100 & 59,600 & 38,800 & 20,800 & 23,400 \\
\hline 17 & $\cdots$ & --- & -.. & -- & -- & --- & 16,900 & 11,600 & 53,100 & 37,800 & 21,900 & 23,400 \\
\hline 18 & --- & --- & --- & --- & --. & -.. & 15,800 & 11,100 & 46,000 & 36,800 & 24,000 & 24,600 \\
\hline 19 & --- & --- & --- & --- & --- & --- & 15,000 & 10,900 & 42,400 & 35,900 & 22,500 & 26,400 \\
\hline 20 & $\ldots$ & --. & --- & --- & --- & --- & 14,600 & 10,900 & 42,400 & 36,400 & 22,200 & 27,900 \\
\hline 21 & --- & --- & --. & --- & --- & --- & 14,100 & 10,900 & 41,100 & 37,300 & 21,600 & 27,000 \\
\hline 22 & -.- & --- & -.- & --- & -.- & --- & 14,100 & 11,100 & 40,500 & 36,400 & 20,800 & 27,000 \\
\hline 23 & --. & --- & ..- & --- & --. & --- & 13,700 & 12,000 & 41,800 & 36,400 & 20,500 & 27,000 \\
\hline 24 & --- & --- & --- & --- & --- & -.. & 13,400 & 12,600 & 41,800 & 37,300 & 20,500 & 27,300 \\
\hline 25 & --- & --- & --. & --. & --. & -- & 13,000 & 14,100 & 38,300 & 39,900 & 20,000 & 27,600 \\
\hline 26 & --- & -- & --- & -- & --- & --- & 12,800 & 15,600 & 35,400 & 42,400 & 19,700 & 28,200 \\
\hline 27 & --- & -.- & --- & --- & -.- & --- & 12,500 & 17,600 & 33,400 & 41,800 & 19,700 & 29,800 \\
\hline 28 & --- & $\cdots$ & --- & --- & --. & --- & 12,100 & 19,700 & 31,500 & 39,900 & 19,700 & 30,400 \\
\hline 29 & --- & -- & --- & $\cdots$ & --- & -- & 12,100 & 20,500 & 29,800 & 38,300 & 19,200 & 30,800 \\
\hline 30 & --- & --- & --- & --- & --- & -- & 11,800 & 20,500 & 31,200 & 36,800 & 19,200 & 29,800 \\
\hline 31 & --- & -- & -- & --- & $\cdots$ & -- & $\cdots$ & 20,500 & --- & 35,900 & 19,200 & -- \\
\hline TOTAL & --- & --- & --- & --- & -- & -- & 593,900 & 408,700 & $1,173,500$ & $1,387,000$ & 711,900 & 715,300 \\
\hline MEAN & --- & -- & --- & --- & $--\cdot$ & --- & 19,800 & 13,180 & 39,120 & 44,740 & 22,960 & 23,840 \\
\hline MAX & --- & $\cdots$ & --- & --- & --- & --- & 41,100 & 20,500 & 63,500 & 78,600 & 33,800 & 30,800 \\
\hline MIN & --- & --- & --- & --- & --- & --- & 11,800 & 10,900 & 20,000 & 33,800 & 19,200 & 18,700 \\
\hline AC-FT & --- & -- & --- & -- & --- & --- & $1,178,000$ & 810,700 & $2,328,000$ & $2,751,000$ & $1,412,000$ & $1,419,000$ \\
\hline
\end{tabular}


Table 16. Daily discharge for the Missouri River at Chamberlain (station 06443000) for the periods September 1928 to September 1929 and April 1945 to September 1954-Continued

[---, no data; MAX, maximum value; MIN, minimum value; AC-FT, acre-feet $]$

\begin{tabular}{|c|c|c|c|c|c|c|c|c|c|c|c|c|}
\hline Day & Oct. & Nov. & Dec. & Jan. & Feb. & Mar. & April & May & June & July & Aug. & Sept. \\
\hline \multicolumn{13}{|c|}{$\begin{array}{c}\text { DISCHARGE, CUBIC FEET PER SECOND, WATER YEAR OCTOBER } 1945 \text { TO SEPTEMBER } 1946 \\
\text { DAILY MEAN VALUES }\end{array}$} \\
\hline 1 & 28,500 & 29,500 & 12,000 & 6,500 & 9,300 & 10,800 & 31,100 & 10,800 & 24,100 & 47,200 & 23,200 & 24,700 \\
\hline 2 & 27,000 & 28,500 & 10,600 & 6,100 & 9,000 & 10,800 & 22,600 & 12,300 & 25,300 & 45,400 & 22,000 & 24,400 \\
\hline 3 & 26,700 & 27,300 & 10,400 & 6,100 & 8,700 & 10,600 & 18,500 & 13,700 & 33,500 & 45,400 & 20,900 & 23,800 \\
\hline 4 & 27,000 & 26,100 & 9,300 & 6,000 & 8,100 & 10,600 & 17,200 & 14,900 & 35,000 & 47,900 & 20,200 & 23,800 \\
\hline 5 & 27,300 & 24,300 & 9,000 & 6,000 & 8,000 & 10,600 & 16,700 & 24,700 & 30,200 & 50,800 & 19,200 & 23,800 \\
\hline 6 & 27,600 & 22,800 & 8,100 & 5,900 & 8,700 & 10,600 & 16,700 & 27,600 & 29,500 & 46,600 & 18,300 & 23,800 \\
\hline 7 & 27,600 & 21,600 & 7,700 & 6,000 & 8,200 & 10,600 & 17,200 & 21,400 & 36,100 & 41,000 & 17,400 & 24,100 \\
\hline 8 & 27,300 & 21,100 & 7,500 & 5,600 & 8,200 & 11,100 & 17,400 & 20,400 & 37,700 & 38,100 & 16,700 & 24,700 \\
\hline 9 & 27,000 & 20,000 & 7,100 & 5,500 & 8,100 & 11,300 & 17,400 & 20,200 & 36,500 & 36,900 & 15,900 & 25,000 \\
\hline 10 & 26,700 & 19,000 & 5,600 & 5,600 & 7,700 & 12,000 & 16,700 & 19,900 & 34,200 & 36,900 & 15,300 & 25,000 \\
\hline 11 & 26,400 & 18,500 & 5,200 & 5,600 & 7,500 & 13,900 & 16,500 & 19,400 & 30,800 & 35,700 & 14,900 & 24,400 \\
\hline 12 & 26,100 & 18,700 & 5,400 & 6,000 & 7,500 & 15,200 & 15,900 & 19,200 & 28,500 & 35,000 & 14,300 & 24,400 \\
\hline 13 & 26,400 & 17,600 & 5,600 & 6,300 & 7,200 & 18,000 & 15,500 & 20,400 & 26,900 & 34,200 & 13,900 & 25,300 \\
\hline 14 & 26,700 & 15,800 & 5,800 & 6,500 & 7,200 & 33,000 & 15,300 & 21,700 & 27,200 & 33,800 & 13,500 & 26,200 \\
\hline 15 & 27,000 & 14,100 & 6,000 & 7,100 & 7,200 & 30,800 & 15,100 & 21,400 & 28,200 & 34,600 & 13,500 & 27,200 \\
\hline 16 & 27,000 & 13,400 & 6,800 & 7,500 & 7,100 & 31,500 & 15,100 & 20,600 & 43,800 & 36,500 & 13,900 & 27,600 \\
\hline 17 & 26,400 & 12,800 & 8,400 & 8,100 & 6,800 & 26,400 & 15,100 & 19,900 & 53,100 & 38,100 & 15,500 & 27,600 \\
\hline 18 & 26,400 & 12,500 & 8,600 & 8,700 & 7,000 & 22,500 & 14,700 & 19,000 & 49,800 & 39,500 & 19,200 & 35,300 \\
\hline 19 & 25,500 & 12,300 & 7,700 & 9,600 & 6,700 & 20,800 & 14,700 & 18,300 & 65,500 & 41,500 & 23,200 & 40,500 \\
\hline 20 & 25,200 & 12,100 & 8,000 & 10,100 & 7,400 & 21,600 & 14,300 & 18,300 & 69,600 & 43,600 & 24,400 & 36,900 \\
\hline 21 & 25,800 & 11,400 & 8,600 & 10,600 & 8,200 & 24,300 & 14,300 & 18,500 & 77,400 & 40,000 & 23,800 & 31,800 \\
\hline 22 & 25,800 & 10,800 & 8,400 & 11,100 & 9,200 & 30,800 & 13,900 & 19,000 & 63,500 & 34,200 & 22,900 & 29,200 \\
\hline 23 & 26,400 & 12,000 & 8,100 & 10,900 & 10,400 & 33,000 & 13,700 & 19,400 & 54,000 & 31,400 & 23,200 & 28,200 \\
\hline 24 & 26,700 & 11,300 & 7,800 & 11,100 & 10,400 & 29,500 & 13,500 & 19,400 & 49,800 & 29,800 & 23,500 & 27,600 \\
\hline 25 & 27,600 & 12,000 & 7,500 & 10,600 & 10,600 & 25,800 & 12,600 & 19,900 & 52,200 & 29,200 & 23,800 & 26,900 \\
\hline 26 & 27,300 & 12,100 & 7,400 & 10,400 & 10,800 & 24,300 & 12,100 & 28,200 & 58,600 & 30,800 & 24,700 & 26,900 \\
\hline 27 & 27,600 & 12,100 & 7,200 & 10,300 & 10,900 & 25,500 & 11,600 & 35,000 & 59,600 & 32,800 & 25,300 & 27,900 \\
\hline 28 & 28,200 & 12,100 & 7,200 & 10,100 & 10,600 & 25,200 & 11,100 & 26,900 & 54,000 & 32,800 & 24,700 & 28,500 \\
\hline 29 & 28,800 & 12,500 & 7,000 & 9,600 & --- & 24,000 & 10,700 & 23,800 & 53,100 & 29,800 & 25,000 & 28,500 \\
\hline 30 & 29,800 & 14,100 & 6,800 & 9,600 & .-- & 32,200 & 10,300 & 23,500 & 49,000 & 27,200 & 25,300 & 27,900 \\
\hline 31 & 29,200 & -.. & 6,700 & 9,300 & --. & 42,400 & --- & 23,200 & -.. & 24,700 & 25,300 & -- \\
\hline TOTAL & 839,000 & 508,400 & 237,500 & 248,400 & 236,700 & 659,700 & 467,500 & 640,900 & $1,316,700$ & $1,151,400$ & 622,900 & 821,900 \\
\hline MEAN & 27,060 & 16,950 & 7,661 & 8,013 & 8,454 & 21,280 & 15,580 & 20,670 & 43,890 & 37,140 & 20,090 & 27,400 \\
\hline MAX & 29,800 & 29,500 & 12,000 & 11,100 & 10,900 & 42,400 & 31,100 & 35,000 & 77,400 & 50,800 & 25,300 & 40,500 \\
\hline MIN & 25,200 & 10,800 & 5,200 & 5,500 & 6,700 & 10,600 & 10,300 & 10,800 & 24,100 & 24,700 & 13,500 & 23,800 \\
\hline AC-FT & $1,664,000$ & $1,008,000$ & 471,100 & 492,700 & 469,500 & $1,309,000$ & 927,300 & $1,271,000$ & $2,612,000$ & $2,284,000$ & $1,236,000$ & $1,630,000$ \\
\hline
\end{tabular}


Table 16. Daily discharge for the Missouri River at Chamberlain (station 06443000) for the periods September 1928 to September 1929 and April 1945 to September 1954-Continued

[---, no data; MAX, maximum value; MIN, minimum value; AC-FT, acre-feet]

\begin{tabular}{|c|c|c|c|c|c|c|c|c|c|c|c|c|}
\hline Day & Oct. & Nov. & Dec. & Jan. & Feb. & Mar. & April & May & June & July & Aug. & Sept. \\
\hline \multicolumn{13}{|c|}{$\begin{array}{c}\text { DISCHARGE, CUBIC FEET PER SECOND, WATER YEAR OCTOBER } 1947 \text { TO SEPTEMBER } 1948 \\
\text { DAILY MEAN VALUES }\end{array}$} \\
\hline 1 & 33,700 & 38,400 & 12,100 & 10,800 & 9,900 & 10,800 & 59,600 & 35,200 & 51,000 & 64,100 & 39,600 & 30,800 \\
\hline 2 & 34,200 & 38,400 & 11,500 & 11,000 & 9,100 & 11,000 & 57,800 & 31,700 & 42,800 & 67,900 & 38,400 & 29,800 \\
\hline 5 & 33,700 & 36,300 & 10,200 & 13,300 & 8,900 & 12,300 & 84,600 & 28,900 & 51,800 & 58,700 & 35,200 & 30,300 \\
\hline 6 & 34,700 & 36,300 & 10,600 & 13,300 & 9,200 & 12,900 & 88,800 & 33,200 & 56,000 & 53,500 & 35,800 & 32,200 \\
\hline 7 & 36,800 & 36,800 & 10,200 & 13,100 & 9,200 & 13,500 & 74,600 & 37,900 & 65,000 & 51,000 & 36,800 & 31,700 \\
\hline 11 & 32,700 & 31,700 & 13,300 & 12,500 & 9,900 & 16,700 & 47,800 & 33,700 & 72,600 & 56,000 & 37,400 & 29,300 \\
\hline 12 & 32,700 & 28,000 & 15,300 & 12,500 & 10,100 & 18,000 & 38,400 & 33,700 & 76,600 & 56,900 & 37,900 & 28,900 \\
\hline 13 & 32,700 & 24,500 & 15,300 & 12,500 & 10,400 & 18,000 & 35,800 & 30,800 & 75,600 & 56,900 & 37,400 & 28,400 \\
\hline 14 & 34,700 & 23,400 & 17,000 & 12,100 & 10,200 & 19,300 & 33,700 & 29,800 & 76,600 & 53,500 & 37,400 & 28,400 \\
\hline 15 & 36,300 & 22,300 & 16,700 & 11,700 & 10,200 & 20,800 & 33,200 & 31,200 & 74,600 & 48,600 & 40,800 & 28,900 \\
\hline 16 & 36,800 & 20,500 & 15,300 & 11,000 & 9,900 & 22,000 & 31,700 & 30,800 & 70,800 & 47,100 & 42,800 & 28,900 \\
\hline 17 & 36,800 & 19,100 & 13,900 & 11,500 & 9,400 & 25,300 & 30,300 & 29,300 & 71,700 & 52,600 & 42,200 & 28,400 \\
\hline 18 & 35,200 & 18,800 & 12,700 & 11,300 & 9,200 & 27,500 & 29,800 & 27,000 & 76,600 & 64,100 & 39,600 & 27,500 \\
\hline 24 & 36,300 & 11,500 & 10,400 & 10,100 & 9,200 & 69,800 & 61,400 & 23,800 & 76,600 & 52,600 & 35,800 & 28,900 \\
\hline 25 & 35,800 & 12,100 & 9,700 & 10,100 & 9,400 & 86,800 & 43,500 & 23,000 & 82,600 & 56,000 & 35,200 & 28,900 \\
\hline 26 & 35,200 & 12,500 & 9,400 & 10,100 & 9,700 & 74,600 & 38,400 & 23,000 & 73,600 & 80,600 & 34,700 & 29,800 \\
\hline 27 & 35,200 & 12,900 & 9,600 & 10,400 & 10,100 & 53,500 & 40,200 & 22,700 & 62,300 & 68,800 & 34,200 & 31,200 \\
\hline 28 & 34,700 & 12,500 & 10,100 & 10,100 & 10,600 & 53,500 & 38,400 & 22,700 & 58,700 & 53,500 & 33,700 & 32,200 \\
\hline 29 & 35,800 & 12,300 & 10,400 & 10,100 & 10,600 & 55,200 & 37,400 & 47,100 & 60,500 & 47,800 & 32,700 & 30,800 \\
\hline 30 & 37,900 & 12,100 & 10,600 & 10,100 & --- & 59,600 & 37,400 & 66,000 & 62,300 & 42,800 & 31,700 & 30,300 \\
\hline 31 & 39,000 & -.- & 11,100 & 10,200 & --- & 62,300 & --- & 60,500 & --- & 40,800 & 31,200 & --- \\
\hline TOTAL & $1,089,600$ & 720,100 & 367,900 & 351,100 & 276,300 & 995,900 & $1,489,400$ & 991,200 & $1,999,500$ & $1,758,600$ & $1,148,700$ & 885,500 \\
\hline MEAN & 35,150 & 24,000 & 11,870 & 11,330 & 9,528 & 32,130 & 49,650 & 31,970 & 66,650 & 56,730 & 37,050 & 29,520 \\
\hline MAX & 39,000 & 38,400 & 17,000 & 13,300 & 10,600 & 86,800 & 88,800 & 66,000 & 82,600 & 80,600 & 44,200 & 32,200 \\
\hline MIN & 32,700 & 11,500 & 9,200 & 9,900 & 8,900 & 10,800 & 29,800 & 22,700 & 41,500 & 40,800 & 31,200 & 27,000 \\
\hline AC-FT & $2,161,000$ & $1,428,000$ & 729,700 & 696,400 & 548,000 & $1,975,000$ & $2,954,000$ & $1,966,000$ & $3,966,000$ & $3,488,000$ & $2,278,000$ & $1,756,000$ \\
\hline
\end{tabular}


Table 16. Daily discharge for the Missouri River at Chamberlain (station 06443000) for the periods September 1928 to September 1929 and April 1945 to September 1954-Continued

[--, no data; MAX, maximum value; MIN, minimum value; AC-FT, acre-feet $]$

\begin{tabular}{|c|c|c|c|c|c|c|c|c|c|c|c|c|}
\hline Day & Oct. & Nov. & Dec. & Jan. & Feb. & Mar. & April & May & June & July & Aug. & Sept. \\
\hline \multicolumn{13}{|c|}{$\begin{array}{c}\text { DISCHARGE, CUBIC FEET PER SECOND, WATER YEAR OCTOBER } 1948 \text { TO SEPTEMBER } 1949 \\
\text { DAILY MEAN VALUES }\end{array}$} \\
\hline 1 & 29,800 & 35,800 & 18,800 & 8,400 & 12,100 & 13,000 & 71,700 & 27,500 & 33,700 & 36,800 & 28,400 & 26,600 \\
\hline 2 & 33,200 & 36,800 & 19,100 & 8,600 & 12,100 & 13,000 & 68,900 & 27.000 & 37,900 & 37,900 & 28,400 & 26,600 \\
\hline 3 & 40,200 & 37,400 & 18,500 & 9,100 & 11,700 & 13,000 & 114,000 & 27,000 & 36,800 & 37,400 & 27,500 & 26,200 \\
\hline 4 & 39,600 & 37,900 & 18,500 & 9,400 & 11,700 & 13,000 & 137,000 & 26,600 & 34,200 & 35,800 & 27,000 & 26,200 \\
\hline 5 & 36,800 & 37,900 & 18,200 & 10,200 & 11,700 & 13,000 & 162,000 & 29,300 & 28,900 & 34,200 & 26,600 & 25,800 \\
\hline 6 & 36,300 & 37,400 & 17,200 & 11,700 & 11,500 & 13,500 & 175,000 & 30,800 & 26,200 & 32,700 & 25,800 & 25,800 \\
\hline 7 & 36,300 & 37,400 & 14,800 & 11,900 & 11,300 & 16,000 & 166,000 & 28,900 & 34,200 & 31,700 & 24,900 & 25,800 \\
\hline \multirow[t]{2}{*}{6} & 37,900 & 38,400 & 8,600 & 11,500 & 11,500 & 19,000 & 164,000 & 26,600 & 29,300 & 31,200 & 24,500 & 25,800 \\
\hline & 40,800 & 40,200 & 6,200 & 11,700 & 12,100 & 25,000 & 141,000 & 25,300 & 40,800 & 30,800 & 24,500 & 25,800 \\
\hline 10 & 40,200 & 40,200 & 5,500 & 12,100 & 12,500 & 32,000 & 95,200 & 25,300 & 42,800 & 28,900 & 24,900 & 25,800 \\
\hline 11 & 39,000 & 38,400 & 4,500 & 12,100 & 13,000 & 36,000 & 73,600 & 25,800 & 39,000 & 28,900 & 25,800 & 25,800 \\
\hline 12 & 37,400 & 35,800 & 4,500 & 12,100 & 13,000 & 37,000 & 64,100 & 26,600 & 36,300 & 28,400 & 26,200 & 26,200 \\
\hline 13 & 36,800 & 33,200 & 4,200 & 11,900 & 13,400 & 37,000 & 54,400 & 26,600 & 34,700 & 29,300 & 25,800 & 26,200 \\
\hline 14 & 36,300 & 31,200 & 4,200 & 12,100 & 13,200 & 37,500 & 47,100 & 26,200 & 35,200 & 28,400 & 24,500 & 26,200 \\
\hline 15 & 36,300 & 27,500 & 4,400 & 12,100 & 13,000 & 37,500 & 39,600 & 26,200 & 34,200 & 27,500 & 25,300 & 26,200 \\
\hline 16 & 36,300 & 24,500 & 5,000 & 11,900 & 13,000 & 35,000 & 35,200 & 25,800 & 31,700 & 28,000 & 24,900 & 26,200 \\
\hline 17 & 35,800 & 21,700 & 5,700 & 11,900 & 12,800 & 30,500 & 33,200 & 26,200 & 31,700 & 29,800 & 25,800 & 26,600 \\
\hline 18 & 36,800 & 19,900 & 6,400 & 12,100 & 13,000 & 26,500 & 29,300 & 26,600 & 42,800 & 30,800 & 26,600 & 27,000 \\
\hline 19 & 36,300 & 18,000 & 6,400 & 12,100 & 12,800 & 25,500 & 28,400 & 25,300 & 50,200 & 29,800 & 28,000 & 27,000 \\
\hline 20 & 36,300 & 18,000 & 6,800 & 11,900 & 12,800 & 24,000 & 27,500 & 23,800 & 52,600 & 28,400 & 28,000 & 27,000 \\
\hline 21 & 35,800 & 17,200 & 5,800 & 11,700 & 12,600 & 23,000 & 27,000 & 24,500 & 57,800 & 27,500 & 26,600 & 28,000 \\
\hline 22 & 36,800 & 17,000 & 5,800 & 11,700 & 12,900 & 21,500 & 24,900 & 24,900 & 52,600 & 25,800 & 26,600 & 28,900 \\
\hline 23 & 36,300 & 17,500 & 5,900 & 11,900 & 12,700 & 20,000 & 24,500 & 25,800 & 48,600 & 24,900 & 27,000 & 28,400 \\
\hline 24 & 35,200 & 18,200 & 6,100 & 11,700 & 12,300 & 25,000 & 27,500 & 27,000 & 44,900 & 25,300 & 26,200 & 27,500 \\
\hline 25 & 33,700 & 18,800 & 6,200 & 11,900 & 11,900 & 55,300 & 31,700 & 42,800 & 42,200 & 26,600 & 24,900 & 26,600 \\
\hline 26 & 32,700 & 19,100 & 6,900 & 12,100 & 12,000 & 110,000 & 31,200 & 46,400 & 40,200 & 28,000 & 25,300 & 25,800 \\
\hline 27 & 32,200 & 19,300 & 7,100 & 11,900 & 12,500 & 70,800 & 29,300 & 46,400 & 40,200 & 28,900 & 25,800 & 24,900 \\
\hline 28 & 33,200 & 19,600 & 6,900 & 11,700 & 12,800 & 60,500 & 27,500 & 42,800 & 39,000 & 28,000 & 25,800 & 24,500 \\
\hline 29 & 33,700 & 20,200 & 7,200 & 11,700 & -.- & 67,900 & 27,000 & 38,400 & 37,900 & 26,600 & 26,600 & 24,100 \\
\hline 30 & 35,800 & 19,100 & 7,200 & 11,700 & -- & 71,700 & 25,800 & 35,200 & 37,400 & 25,800 & 27,000 & 24,100 \\
\hline 31 & 35,800 & -- & 7,800 & 11,900 & $\ldots$ & 80,600 & -- & 33,200 & -- & 26,600 & 27,000 & -.. \\
\hline TOTAL & $1,119,600$ & 833,600 & 270,400 & 354,700 & 347,900 & $1,103,300$ & $2,003,600$ & 920,800 & $1,174,000$ & 920,700 & 812,200 & 787,600 \\
\hline MEAN & 36,120 & 27,790 & 8,723 & 11,440 & 12,420 & 35,590 & 66,790 & 29,700 & 39,130 & 29,700 & 26,200 & 26,250 \\
\hline MAX & 40,800 & 40,200 & 19,100 & 12,100 & 13,400 & 110,000 & 175,000 & 46,400 & 57,800 & 37,900 & 28,400 & 28,900 \\
\hline MIN & 29,800 & 17,000 & 4,200 & 8,400 & 11,300 & 13,000 & 24,500 & 23,800 & 26,200 & 24,900 & 24,500 & 24,100 \\
\hline AC-FT & $2,221,000$ & $1,653,000$ & 536,300 & 703,500 & 690,100 & $2,188,000$ & $3,974,000$ & $1,826,000$ & $2,329,000$ & $1,826,000$ & $1,611,000$ & $1,562,000$ \\
\hline
\end{tabular}


Table 16. Daily discharge for the Missouri River at Chamberlain (station 06443000) for the periods September 1928 to September 1929 and April 1945 to September 1954-Continued

[---, no data; MAX, maximum value; MIN, minimum value; AC-FT, acre-feet]

\begin{tabular}{|c|c|c|c|c|c|c|c|c|c|c|c|c|}
\hline Day & Oct. & Nov. & Dec. & Jan. & Feb. & Mar. & April & May & June & July & Aug. & Sept. \\
\hline \multicolumn{13}{|c|}{$\begin{array}{l}\text { DISCHARGE, CUBIC FEET PER SECOND, WATER YEAR OCTOBER } 1949 \text { TO SEPTEMBER } 1950 \\
\text { DAILY MEAN VALUES }\end{array}$} \\
\hline 1 & 24,500 & 28,900 & 14,600 & 6,500 & 8,100 & 8,300 & 65,400 & 46,700 & 27,000 & 72,600 & 27,000 & 28,900 \\
\hline 2 & 24,500 & 26,600 & 14,600 & 6,500 & 8,400 & 8,100 & 124,000 & 43,900 & 26,600 & 64,100 & 27,000 & 28,900 \\
\hline 3 & 24,900 & 25,800 & 14,400 & 6,500 & 8,400 & 8,100 & 92,500 & 42,400 & 25,100 & 53,500 & 26,200 & 28,900 \\
\hline 4 & 24,900 & 25,300 & 14,200 & 6,500 & 8,400 & 7,500 & 78,200 & 41,100 & 24,200 & 49,400 & 25,800 & 28,400 \\
\hline 5 & 24,900 & 23,800 & 13,700 & 6,600 & 8,400 & 7,500 & 71,800 & 40,500 & 25,100 & 45,600 & 26,600 & 28,900 \\
\hline 6 & 25,300 & 22,700 & 13,900 & 7,200 & 8,300 & 8,700 & 88,000 & 40,600 & 27,500 & 40,200 & 26,600 & 28,400 \\
\hline 7 & 24,900 & 21,700 & 13,300 & 7,600 & 8,100 & 12,700 & 85,000 & 41,400 & 28,200 & 37,400 & 27,000 & 27,500 \\
\hline 8 & 24,500 & 21,100 & 13,100 & 8,000 & 8,000 & 14,800 & 88,200 & 46,000 & 27,600 & 37,400 & 27,000 & 27,000 \\
\hline 9 & 24,900 & 20,200 & 12,900 & 8,100 & 8,100 & 14,200 & 82,500 & 65,200 & 27,300 & 39,000 & 27,500 & 26,600 \\
\hline 10 & 26,600 & 19,600 & 11,700 & 8,400 & 8,100 & 12,700 & 89,600 & 63,400 & 27,300 & 41,500 & 28,900 & 27,000 \\
\hline 11 & 26,600 & 18,800 & 11,300 & 8,400 & 8,300 & 11,300 & 78,100 & 59,100 & 27,300 & 47,800 & 30,300 & 27,500 \\
\hline 12 & 28,400 & 18,200 & 8,100 & 8,400 & 8,100 & 10,200 & 65,800 & 59,900 & 27,200 & 51,000 & 30,800 & 27,500 \\
\hline 13 & 28,000 & 17,700 & 8,100 & 8,300 & 8,300 & 9,600 & 59,700 & 54,300 & 27,200 & 47,800 & 29,300 & 27,500 \\
\hline 14 & 26,200 & 17,200 & 6,600 & 8,100 & 7,800 & 9,400 & 53,700 & 47,800 & 28,000 & 46,400 & 28,000 & 27,000 \\
\hline 15 & 25,800 & 16,700 & 6,300 & 7,800 & 8,000 & 9,400 & 68,800 & 45,200 & 35,700 & 44,200 & 28,400 & 27,000 \\
\hline 16 & 26,600 & 16,500 & 5,500 & 7,800 & 8,600 & 9,400 & 107,000 & 47,200 & 51,700 & 44,200 & 28,400 & 27,000 \\
\hline 17 & 27,000 & 16,200 & 5,500 & 8,100 & 8,600 & 10,200 & 126,000 & 47,000 & 51,700 & 44,200 & 31,200 & 28,400 \\
\hline 18 & 27,000 & 16,000 & 5,500 & 8,000 & 8,700 & 10,800 & 158,000 & 43,500 & 45,900 & 44,200 & 37,900 & 29,800 \\
\hline 19 & 27,500 & 16,000 & 5,000 & 8,100 & 8,600 & 11,500 & 181,000 & 41,500 & 39,800 & 43,500 & 38,400 & 31,700 \\
\hline 20 & 26,600 & 16,000 & 5,100 & 7,800 & 8,400 & 11,700 & 212,000 & 36,600 & 36,000 & 42,200 & 36,300 & 32,200 \\
\hline 21 & 26,600 & 15,800 & 4,900 & 8,100 & 8,300 & 11,700 & 239,000 & 34,800 & 33,900 & 42,200 & 35,200 & 33,200 \\
\hline 22 & 26,600 & 15,500 & 5,000 & 8,000 & 8,000 & 12,100 & 245,000 & 34,500 & 35,500 & 41,500 & 34,700 & 44,200 \\
\hline 23 & 25,800 & 15,300 & 4,400 & 7,800 & 8,000 & 12,500 & 190,000 & 33,000 & 52,500 & 40,200 & 33,200 & 39,000 \\
\hline 24 & 24,900 & 15,000 & 4,600 & 6,900 & 7,800 & 14,200 & 115,000 & 30,800 & 59,100 & 37,900 & 31,200 & 36,800 \\
\hline 25 & 25,300 & 14,600 & 5,000 & 7,100 & 8,000 & 20,200 & 87,800 & 29,900 & 63,200 & 35,800 & 29,800 & 35,800 \\
\hline 26 & 25,800 & 14,600 & 5,100 & 7,200 & 8,000 & 37,400 & 74,100 & 29,100 & 67,300 & 34,700 & 29,800 & 34,700 \\
\hline 27 & 28,000 & 14,600 & 5,100 & 7,200 & 8,000 & 79,600 & 62,100 & 27,600 & 64,100 & 33,200 & 30,300 & 32,700 \\
\hline 28 & 31,200 & 14,600 & 5,100 & 7,300 & 8,700 & 89,900 & 54,100 & 26,600 & 56,900 & 31,200 & 29,800 & 30,800 \\
\hline 29 & 33,200 & 14,600 & 5,300 & 7,400 & -.. & 62,300 & 49,600 & 26,600 & 54,000 & 29,800 & 29,800 & 29,800 \\
\hline 30 & 31,700 & 14,600 & 5,300 & 7,700 & --- & 33,700 & 48,900 & 27,000 & 59,400 & 28,400 & 29,800 & 29,800 \\
\hline 31 & 29,300 & --. & 6,100 & 8,000 & --- & 42,800 & --- & 27,100 & -- & 28,000 & 29,300 & $\cdots$ \\
\hline TOTAL & 828,000 & 554,200 & 259,300 & 235,400 & 230,500 & 622,500 & $3,140,900$ & $1,280,300$ & $1,182,300$ & $1,319,100$ & 931,500 & 912,900 \\
\hline MEAN & 26,710 & 18,470 & 8,365 & 7,594 & 8,232 & 20,080 & 104,700 & 41,300 & 39,410 & 42,550 & 30,050 & 30,430 \\
\hline MAX & 33,200 & 28,900 & 14,600 & 8,400 & 8,700 & 89,900 & 245,000 & 65,200 & 67,300 & 72,600 & 38,400 & 44,200 \\
\hline MIN & 24,500 & 14,600 & 4,400 & 6,500 & 7,800 & 7,500 & 48,900 & 26,600 & 24,200 & 28,000 & 25,800 & 26,600 \\
\hline AC-FT & $1,642,000$ & $1,099,000$ & 514,300 & 466,900 & 457,200 & $1,235,000$ & $6,230,000$ & $2,539,000$ & $2,345,000$ & $2,616,000$ & $1,848,000$ & $1,811,000$ \\
\hline
\end{tabular}


Table 16. Daily discharge for the Missouri River at Chamberlain (station 06443000) for the periods September 1928 to September 1929 and April 1945 to September 1954-Continued

[---, no data; MAX, maximum value; MIN, minimum value; AC-FT, acre-feet $]$

\begin{tabular}{|c|c|c|c|c|c|c|c|c|c|c|c|c|}
\hline Day & Oct. & Nov. & Dec. & Jan. & Feb. & Mar. & April & May & June & July & Aug. & Sept. \\
\hline \multicolumn{13}{|c|}{$\begin{array}{c}\text { DISCHARGE, CUBIC FEET PER SECOND, WATER YEAR OCTOBER } 1950 \text { TO SEPTEMBER } 1951 \\
\text { DAILY MEAN VALUES }\end{array}$} \\
\hline 1 & 29,400 & 34,100 & 8,000 & 16,300 & 14,400 & 14,000 & 48,600 & 27,300 & 39,600 & 50,800 & 32,100 & 33,600 \\
\hline 2 & 29,400 & 34,100 & 8,000 & 16,800 & 15,300 & 14,200 & 52,300 & 26,900 & 43,900 & 48,600 & 33,100 & 37,900 \\
\hline 3 & 28,200 & 34,600 & 7,500 & 17,000 & 15,100 & 15,300 & 48,600 & 26,600 & 53,800 & 48,600 & 34,100 & 53,800 \\
\hline 4 & 27,700 & 34,600 & 6,500 & 17,300 & 15,100 & 16,300 & 50,000 & 26,600 & 45,800 & 45,800 & 34,100 & 48,600 \\
\hline 5 & 28,200 & 34,600 & 5,600 & 17,500 & 14,400 & 17,000 & 67,400 & 26,200 & 42,600 & 39,600 & 33,600 & 42,600 \\
\hline 6 & 29,400 & 34,100 & 5,300 & 18,100 & 14,200 & 18,100 & 95,600 & 26,200 & 57,000 & 39,000 & 31,700 & 42,000 \\
\hline 7 & 30,700 & 33,100 & 6,500 & 18,300 & 14,400 & 18,100 & 103,000 & 26,600 & 59,500 & 36,200 & 30,700 & 43,900 \\
\hline 8 & 31,200 & 36,800 & 6,600 & 18,300 & 14,200 & 18,100 & 108,000 & 27,700 & 56,200 & 34,600 & 30,700 & 43,900 \\
\hline 9 & 31,700 & 39,600 & 7,500 & 18,100 & 13,300 & 18,100 & 91,000 & 30,300 & 62,100 & 33,600 & 32,100 & 43,200 \\
\hline 10 & 33,600 & 38,400 & 8,500 & 17,800 & 12,900 & 18,100 & 92,800 & 32,100 & 54,600 & 32,600 & 34,100 & 43,200 \\
\hline 11 & 33,600 & 35,700 & 9,200 & 17,000 & 12,500 & 17,000 & 103,000 & 34,100 & 50,800 & 33,600 & 36,800 & 42,600 \\
\hline 12 & 32,600 & 34,100 & 10,200 & 16,800 & 12,100 & 16,500 & 90,000 & 32,100 & 45,200 & 35,700 & 37,900 & 43,900 \\
\hline 13 & 32,100 & 31,200 & 10,700 & 16,000 & 12,300 & 15,500 & 75,500 & 31,700 & 40,800 & 34,600 & 38,400 & 40,800 \\
\hline 14 & 32,100 & 27,700 & 11,500 & 16,500 & 12,300 & 14,800 & 79,100 & 31,200 & 37,300 & 33,600 & 38,400 & 38,400 \\
\hline 15 & 31,200 & 25,800 & 12,300 & 16,800 & 12,100 & 14,000 & 71,900 & 30,300 & 35,200 & 34,100 & 37,300 & 35,700 \\
\hline 16 & 31,200 & 23,900 & 13,800 & 15,500 & 11,700 & 13,100 & 69,200 & 28,200 & 34,100 & 36,200 & 35,700 & 34,100 \\
\hline 17 & 30,700 & 23,200 & 16,000 & 15,300 & 11,500 & 12,700 & 50,800 & 26,600 & 33,100 & 37,300 & 37,300 & 34,600 \\
\hline 18 & 30,300 & 22,200 & 17,500 & 15,100 & 11,300 & 12,300 & 40,200 & 25,800 & 33,100 & 39,000 & 40,200 & 35,200 \\
\hline 19 & 29,800 & 20,200 & 17,300 & 14,200 & 11,100 & 12,100 & 36,200 & 25,800 & 34,600 & 36,800 & 42,600 & 35,700 \\
\hline 20 & 28,600 & 18,600 & 16,000 & 14,400 & 11,100 & 11,900 & 36,200 & 26,600 & 33,100 & 35,200 & 43,900 & 35,700 \\
\hline 21 & 28,600 & 16,300 & 14,800 & 13,300 & 11,100 & 11,300 & 34,600 & 29,000 & 31,200 & 34,600 & 41,400 & 36,200 \\
\hline 22 & 29,000 & 14,400 & 14,800 & 12,700 & 11,100 & 10,900 & 32,600 & 30,300 & 30,300 & 35,200 & 38,400 & 35,200 \\
\hline 23 & 30,300 & 11,100 & 14,800 & 12,300 & 11,500 & 11,100 & 30,300 & 29,800 & 32,100 & 36,800 & 36,800 & 34,100 \\
\hline 24 & 32,600 & 9,000 & 14,200 & 11,900 & 11,500 & 10,900 & 29,800 & 29,800 & 37,300 & 36,200 & 35,700 & 32,600 \\
\hline 25 & 34,100 & 8,000 & 13,500 & 12,700 & 11,900 & 11,100 & 29,800 & 30,700 & 62,100 & 34,600 & 36,200 & 31,700 \\
\hline 26 & 34,600 & 7,500 & 13,100 & 12,500 & 11,900 & 12,000 & 29,800 & 32,600 & 64,700 & 33,600 & 36,800 & 32,600 \\
\hline 27 & 34,600 & 7,000 & 13,500 & 12,100 & 12,300 & 15,000 & 29,800 & 31,200 & 61,200 & 33,100 & 36,800 & 33,100 \\
\hline 28 & 35,200 & 7,000 & 14,000 & 13,800 & 12,900 & 19,200 & 29,000 & 29,000 & 52,300 & 32,600 & 36,200 & 32,100 \\
\hline 29 & 35,700 & 7,000 & 14,400 & 13,800 & -.. & 21,800 & 26,200 & 28,200 & 49,300 & 32,600 & 35,700 & 33,100 \\
\hline 30 & 35,200 & 7,500 & 14,800 & 13,500 & $\cdots$ & 26,600 & 26,200 & 28,600 & 50,000 & 32,100 & 35,200 & 34,600 \\
\hline 31 & 34,100 & -. & 15,500 & 14,200 & $\cdots$ & 57,000 & -.- & 33,600 & -- & 32,100 & 34,100 & -- \\
\hline TOTAL & 975,700 & 711,400 & 361,900 & 475,900 & 355,500 & 514,100 & $1,707,500$ & 901,700 & $1,362,900$ & $1,139,000$ & $1,118,100$ & $1,144,700$ \\
\hline MEAN & 31,470 & 23,710 & 11,670 & 15,350 & 12,700 & 16,580 & 56,920 & 29,090 & 45,430 & 36,740 & 36,070 & 38,160 \\
\hline MAX & 35,700 & 39,600 & 17,500 & 18,300 & 15,300 & 57,000 & 108,000 & 34,100 & 64,700 & 50,800 & 43,900 & 53,800 \\
\hline MIN & 27,700 & 7,000 & 5,300 & 11,900 & 11,100 & 10,900 & 26,200 & 25,800 & 30,300 & 32,100 & 30,700 & 31,700 \\
\hline AC-FT & $1,935,000$ & $1,411,000$ & 717,800 & 943,900 & 705,100 & $1,020,000$ & $3,387,000$ & $1,789,000$ & $2,703,000$ & $2,259,000$ & $2,218,000$ & $2,271,000$ \\
\hline
\end{tabular}


Table 16. Daily discharge for the Missouri River at Chamberlain (station 06443000) for the periods September 1928 to September 1929 and April 1945 to September 1954-Continued

[---, no data; MAX, maximum value; MIN, minimum value; AC-FT, acre-feet]

\begin{tabular}{|c|c|c|c|c|c|c|c|c|c|c|c|c|}
\hline Day & Oct. & Nov. & Dec. & Jan. & Feb. & Mar. & April & May & June & July & Aug. & Sept. \\
\hline \multicolumn{13}{|c|}{$\begin{array}{c}\text { DISCHARGE, CUBIC FEET PER SECOND, WATER YEAR OCTOBER } 1951 \text { TO SEPTEMBER } 1952 \\
\text { DAILY MEAN VALUES }\end{array}$} \\
\hline 1 & 40,800 & 39,000 & 10,400 & 8,500 & 16,000 & 18,300 & 23,000 & 50,600 & 51,200 & 35,500 & 22,900 & 28,300 \\
\hline 2 & 43,200 & 39,000 & 10,500 & 8,800 & 16,000 & 17,300 & 53,000 & 48,500 & 51,800 & 34,200 & 22,000 & 28,300 \\
\hline 3 & 42,000 & 39,600 & 18,000 & 8,600 & 16,300 & 16,300 & 88,000 & 46,500 & 46,900 & 33,300 & 21,300 & 28,600 \\
\hline 4 & 41,400 & 37,900 & 51,700 & 8,000 & 16,300 & 15,000 & 103,000 & 45,100 & 43,600 & 32,900 & 20,900 & 28,300 \\
\hline 5 & 44,600 & 40,200 & 26,600 & 8,200 & 15,800 & 13,800 & 162,000 & 44,200 & 43,600 & 30,300 & 21,300 & 28,600 \\
\hline 6 & 45,200 & 39,000 & 21,500 & 8,600 & 15,800 & 13,200 & 202,000 & 42,000 & 45,800 & 29,900 & 22,400 & 29,200 \\
\hline 7 & 37,900 & 35,200 & 20,500 & 9,000 & 15,800 & 12,900 & 249,000 & 41,200 & 45,800 & 30,600 & 23,700 & 29,900 \\
\hline 8 & 36,200 & 36,800 & 20,000 & 9,500 & 16,500 & 12,700 & 272,000 & 43,700 & 46,900 & 31,300 & 25,000 & 29,200 \\
\hline 9 & 37,300 & 37,900 & 19,000 & 10,000 & 17,000 & 12,900 & 326,000 & 51,900 & 44,600 & 31,000 & 25,300 & 28,300 \\
\hline 10 & 39,000 & 40,800 & 18,000 & 10,500 & 17,500 & 13,100 & 398,000 & 54,500 & 42,500 & 29,900 & 25,300 & 27,900 \\
\hline 11 & 39,600 & 41,400 & 16,500 & 10,700 & 17,500 & 13,100 & 434,000 & 50,000 & 39,300 & 28,900 & 25,600 & 27,300 \\
\hline 12 & 39,600 & 40,200 & 14,000 & 11,700 & 18,000 & 13,100 & 413,000 & 47,900 & 39,300 & 27,900 & 26,100 & 26,400 \\
\hline 13 & 39,600 & 36,200 & 10,000 & 12,500 & 18,500 & 13,500 & 339,000 & 48,500 & 43,600 & 26,700 & 27,000 & 25,800 \\
\hline 14 & 38,400 & 36,200 & 6,000 & 13,300 & 19,000 & 13,800 & 260,000 & 52,500 & 48,700 & 26,100 & 27,900 & 25,600 \\
\hline 15 & 36,800 & 36,200 & 5,000 & 14,600 & 19,500 & 13,800 & 180,000 & 54,000 & 55,000 & 25,600 & 28,300 & 25,800 \\
\hline 16 & 36,800 & 36,200 & 4,500 & 15,500 & 20,500 & 13,800 & 125,000 & 49,300 & 70,000 & 24,700 & 29,200 & 26,100 \\
\hline 17 & 37,900 & 36,800 & 4,500 & 16,300 & 21,500 & 15,000 & 106,000 & 43,200 & 67,000 & 23,400 & 29,600 & 27,000 \\
\hline 18 & 40,800 & 36,200 & 4,500 & 16,800 & 21,500 & 16,000 & 95,300 & 39,700 & 59,800 & 22,700 & 30,300 & 27,300 \\
\hline 19 & 41,400 & 35,200 & 4,500 & 17,300 & 20,500 & 17,000 & 87,500 & 37,600 & 53,000 & 22,700 & 31,000 & 27,300 \\
\hline 20 & 40,800 & 36,200 & 4,800 & 17,500 & 20,000 & 17,000 & 80,500 & 36,400 & 51,200 & 22,000 & 31,700 & 27,300 \\
\hline 21 & 40,200 & 36,800 & 5,300 & 17,500 & 19,500 & 16,500 & 78,800 & 33,300 & 49,300 & 21,300 & 32,100 & 27,600 \\
\hline 22 & 41,400 & 35,200 & 5,300 & 17,500 & 19,500 & 16,000 & 76,100 & 31,000 & 45,800 & 20,500 & 31,000 & 27,300 \\
\hline 23 & 43,200 & 33,100 & 5,500 & 16,500 & 19,000 & 15,000 & 75,200 & 29,600 & 42,500 & 20,100 & 29,900 & 27,300 \\
\hline 24 & 42,000 & 31,700 & 6,300 & 15,800 & 19,000 & 14,500 & 72,500 & 28,800 & 41,400 & 19,700 & 29,200 & 27,300 \\
\hline 25 & 39,000 & 30,700 & 7,000 & 15,500 & 19,000 & 14,000 & 75,600 & 30,100 & 38,300 & 19,700 & 28,900 & 27,000 \\
\hline 26 & 37,900 & 23,900 & 7,200 & 14,800 & 19,000 & 14,000 & 79,000 & 37,600 & 36,900 & 20,700 & 28,900 & 27,000 \\
\hline 27 & 37,900 & 16,500 & 7,700 & 14,600 & 19,000 & 14,000 & 72,500 & 38,300 & 34,200 & 23,200 & 28,900 & 27,300 \\
\hline 28 & 38,400 & 12,500 & 7,800 & 15,300 & 18,900 & 15,000 & 62,800 & 36,400 & 32,900 & 25,000 & 28,600 & 27,000 \\
\hline 29 & 39,000 & 11,100 & 8,200 & 15,300 & 18,900 & 18,000 & 56,900 & 33,800 & 42,500 & 25,800 & 28,300 & 26,700 \\
\hline 30 & 37,900 & 10,500 & 8,300 & 15,500 & --- & 21,000 & 53,200 & 31,300 & 45,800 & 25,600 & 27,900 & 26,700 \\
\hline 31 & 38,400 & -- & 8,200 & 16,000 & --- & 24,000 & --. & 36,700 & -- & 24,500 & 27,600 & -- \\
\hline TOTAL & $1,234,600$ & 998,200 & 367,300 & 410,200 & 531,300 & 473,600 & $4,698,900$ & $1,294,200$ & $1,399,200$ & 815,700 & 838,100 & 823,700 \\
\hline MEAN & 39,830 & 33,270 & 11,850 & 13,230 & 18,320 & 15,280 & 156,600 & 41,750 & 46,640 & 26,310 & 27,040 & 27,460 \\
\hline MAX & 45,200 & 41,400 & 51,700 & 17,500 & 21,500 & 24,000 & 434,000 & 54,500 & 70,000 & 35,500 & 32,100 & 29,900 \\
\hline MIN & 36,200 & 10,500 & 4,500 & 8,000 & 15,800 & 12,700 & 23,000 & 28,800 & 32,900 & 19,700 & 20,900 & 25,600 \\
\hline AC-FT & $2,449,000$ & $1,980,000$ & 728,500 & 813,600 & $1,054,000$ & 939,400 & $9,320,000$ & $2,567,000$ & $2,775,000$ & $1,618,000$ & $1,662,000$ & $1,634,000$ \\
\hline
\end{tabular}


Table 16. Daily discharge for the Missouri River at Chamberlain (station 06443000) for the periods September 1928 to September 1929 and April 1945 to September 1954-Continued

[---, no data; MAX, maximum value; MIN, minimum value; AC-FT, acre-feet]

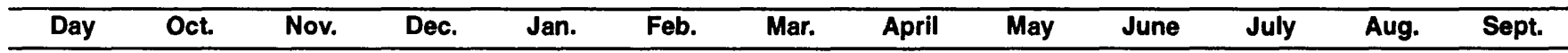

DISCHARGE, CUBIC FEET PER SECOND, WATER YEAR OCTOBER 1952 TO SEPTEMBER 1953 DAILY MEAN VALUES

\begin{tabular}{|c|c|c|c|c|c|c|c|c|c|c|c|c|}
\hline 1 & 26,800 & 25,600 & 7,000 & 11,500 & 12,600 & 11,000 & 36,100 & 21,200 & 21,900 & 59,200 & 28,500 & 28,300 \\
\hline 2 & 26,600 & 25,800 & 7,000 & 11,500 & 12,200 & 11,000 & 30,900 & 36,700 & 24,400 & 53,200 & 28,000 & 28,400 \\
\hline 3 & 26,300 & 26,600 & 7,500 & 12,000 & 12,500 & 11,000 & 23,100 & 51,000 & 37,000 & 48,500 & 29,100 & 28,700 \\
\hline 4 & 26,300 & 26,900 & 8,000 & 12,000 & 12,800 & 11,000 & 21,100 & 56,300 & 36,600 & 47,100 & 34,100 & 27,900 \\
\hline 5 & 26,300 & 26,900 & 8,500 & 12,000 & 13,000 & 10,500 & 20,400 & 50,100 & 31,500 & 46,000 & 33,000 & 26,800 \\
\hline 6 & 26,800 & 26,700 & 8,500 & 11,500 & 13,000 & 10,500 & 20,200 & 43,700 & 27,500 & 44,400 & 32,100 & 26,500 \\
\hline 7 & 27,100 & 26,800 & 8,000 & 11,200 & 13,000 & 10,500 & 20,500 & 37,900 & 25,000 & 41,500 & 32,900 & 26,700 \\
\hline 8 & 27,400 & 27,400 & 8,000 & 11,200 & 13,000 & 10,500 & 22,400 & 34,100 & 41,200 & 38,900 & 32,000 & 26,900 \\
\hline 9 & 27,900 & 27,600 & 8,000 & 11,200 & 13,000 & 10,500 & 39,700 & 32,200 & 62,000 & 37,300 & 30,900 & 27,000 \\
\hline 10 & 28,200 & 28,500 & 8,500 & 11,200 & 13,000 & 10,500 & 41,500 & 31,100 & 61,500 & 36,500 & 30,700 & 27,000 \\
\hline 11 & 28,200 & 28,600 & 9,000 & 11,000 & 12,900 & 11,000 & 36,000 & 30,100 & 61,900 & 35,600 & 31,000 & 27,800 \\
\hline 12 & 28,500 & 27,700 & 9,000 & 11,000 & 12,500 & 12,000 & 29,400 & 27,600 & 60,500 & 35,300 & 30,300 & 29,100 \\
\hline 13 & 28,900 & 25,800 & 8,000 & 11,000 & 12,500 & 15,000 & 22,700 & 26,700 & 50,800 & 35,300 & 30,800 & 30,200 \\
\hline 14 & 29,100 & 23,900 & 7,000 & 11,000 & 12,500 & 16,000 & 19,500 & 26,000 & 46,100 & 34,600 & 30,800 & 31,200 \\
\hline 15 & 29,100 & 22,000 & 6,500 & 10,800 & 12,400 & 25,000 & 18,200 & 25,700 & 45,500 & 33,400 & 30,800 & 31,100 \\
\hline 16 & 28,800 & 21,000 & 6,000 & 10,800 & 12,200 & 32,000 & 17,000 & 25,400 & 66,000 & 32,000 & 31,000 & 30,700 \\
\hline 17 & 28,900 & 20,200 & 6,000 & 11,300 & 11,800 & 28,000 & 16,300 & 25,200 & 95,000 & 30,900 & 30,900 & 30,500 \\
\hline 18 & 28,800 & 19,900 & 6,000 & 11,400 & 11,500 & 24,000 & 16,000 & 25,100 & 103,000 & 30,100 & 29,700 & 30,000 \\
\hline 19 & 28,300 & 19,200 & 7,000 & 11,200 & 11,500 & 28,000 & 15,700 & 26,500 & 93,200 & 30,300 & 28,000 & 29,500 \\
\hline 20 & 27,800 & 18,700 & 9,000 & 11,500 & 11,500 & 41,000 & 15,300 & 29,100 & 76,000 & 30,200 & 27,000 & 29,200 \\
\hline 21 & 27,900 & 18,100 & 10,500 & 12,200 & 12,000 & 51,000 & 14,900 & 30,900 & 79,000 & 29,100 & 26,400 & 28,800 \\
\hline 22 & 27,700 & 17,700 & 11,500 & 12,600 & 12,000 & 48,000 & 14,500 & 28,800 & 102,000 & 28,200 & 25,900 & 28,900 \\
\hline 23 & 27,800 & 17,500 & 13,000 & 12,300 & 12,000 & 51,000 & 14,000 & 25,600 & 110,000 & 27,100 & 25,900 & 30,100 \\
\hline 24 & 28,300 & 17,300 & 14,000 & 12,000 & 12,000 & 49,700 & 14,000 & 23,200 & 102,000 & 26,800 & 26,400 & 31,000 \\
\hline 25 & 29,400 & 16,600 & 14,000 & 12,000 & 12,000 & 42,000 & 14,100 & 23,400 & 93,700 & 26,000 & 26,500 & 30,400 \\
\hline 26 & 29,500 & 15,500 & 14,500 & 12,000 & 11,500 & 37,100 & 14,100 & 22,600 & 89,600 & 26,000 & 27,300 & 29,800 \\
\hline 27 & 29,500 & 12,000 & 14,500 & 12,200 & 11,500 & 33,100 & 14,100 & 22,100 & 87,800 & 26,700 & 27,900 & 29,300 \\
\hline 28 & 28,500 & 10,000 & 13,500 & 12,500 & 11,000 & 29,300 & 14,300 & 22,500 & 82,800 & 28,300 & 28,700 & 28,700 \\
\hline 29 & 27,400 & 9,000 & 12,500 & 12,500 & -- & 27,200 & 15,000 & 23,000 & 74,300 & 28,700 & 28,900 & 29,500 \\
\hline 30 & 26,800 & 8,000 & 12,000 & 12,500 & --- & 26,900 & 16,700 & 23,200 & 66,000 & 29,100 & 29,100 & 30,200 \\
\hline 31 & 25,900 & --- & 11,500 & 12,500 & --- & 27,600 & -- & 22,800 & -- & 29,000 & 28,600 & -- \\
\hline TOTAL & 864,800 & 637,500 & 294,000 & 361,600 & 343,400 & 761,900 & 627,700 & 929,800 & $1,953,800$ & $1,085,300$ & 913,200 & 870,200 \\
\hline MEAN & 27,900 & 21,250 & 9,484 & 11,660 & 12,260 & 24,580 & 20,920 & 29,990 & 65,130 & 35,010 & 29,460 & 29,010 \\
\hline MAX & 29,500 & 28,600 & 14,500 & 12,600 & 13,000 & 51,000 & 41,500 & 56,300 & 110,000 & 59,200 & 34,100 & 31,200 \\
\hline MIN & 25,900 & 8,000 & 6,000 & 10,800 & 11,000 & 10,500 & 14,000 & 21,200 & 21,900 & 26,000 & 25,900 & 26,500 \\
\hline AC-FT & $1,715,000$ & $1,264,000$ & 583,100 & 717,200 & 681,100 & $1,511,000$ & $1,245,000$ & $1,844,000$ & $3,875,000$ & $2,153,000$ & $1,811,000$ & $1,726,000$ \\
\hline
\end{tabular}


Table 16. Daily discharge for the Missouri River at Chamberlain (station 06443000) for the periods September 1928 to September 1929 and April 1945 to September 1954-Continued

[--, no data; MAX, maximum value; MIN, minimum value; AC-FT, acre-feet $]$

\begin{tabular}{|c|c|c|c|c|c|c|c|c|c|c|c|c|}
\hline Day & Oct. & Nov. & Dec. & Jan. & Feb. & Mar. & April & May & June & July & Aug. & Sept. \\
\hline \multicolumn{13}{|c|}{$\begin{array}{c}\text { DISCHARGE, CUBIC FEET PER SECOND, WATER YEAR OCTOBER } 1953 \text { TO SEPTEMBER } 1954 \\
\text { DAILY MEAN VALUES }\end{array}$} \\
\hline 1 & 31,400 & 40,800 & 17,000 & 8,500 & 9,700 & 13,500 & 15,800 & 28,000 & 28,400 & 12,600 & 38,800 & 38,500 \\
\hline 2 & 31,300 & 37,700 & 16,900 & 8,500 & 9,700 & 13,000 & 15,100 & 28,300 & 29,500 & 13,100 & 38,800 & 38,700 \\
\hline 3 & 30,800 & 34,900 & 16,700 & 8,500 & 9,800 & 12,000 & 14,300 & 28,600 & 31,100 & 13,400 & 39,600 & 38,800 \\
\hline 4 & 30,800 & 32,900 & 16,600 & 8,700 & 9,800 & 10,000 & 13,400 & 28,800 & 31,000 & 13,700 & 39,800 & 38,800 \\
\hline 5 & 30,700 & 31,700 & 16,300 & 8,800 & 9,800 & 10,400 & 13,000 & 28,400 & 28,300 & 14,100 & 39,200 & 39,200 \\
\hline 6 & 30,400 & 31,200 & 16,000 & 8,600 & 9,800 & 15,000 & 12,700 & 27,600 & 26,600 & 14,200 & 39,100 & 39,400 \\
\hline 7 & 30,700 & 31,200 & 15,100 & 8,500 & 9,800 & 22,200 & 12,600 & 27,300 & 28,400 & 14,100 & 38,800 & 39,900 \\
\hline 8 & 31,300 & 31,200 & 14,200 & 8,500 & 9,800 & 26,000 & 13,100 & 26,600 & 28,400 & 13,800 & 38,500 & 39,900 \\
\hline 9 & 31,200 & 30,900 & 13,200 & 8,000 & 9,800 & 21,900 & 13,800 & 26,100 & 26,200 & 15,000 & 38,300 & 39,700 \\
\hline 10 & 31,300 & 30,400 & 9,000 & 7,500 & 10,000 & 20,900 & 19,800 & 25,800 & 25,700 & 16,200 & 38,200 & 40,400 \\
\hline 11 & 31,100 & 29,900 & 10,000 & 7,000 & 10,000 & 20,500 & 26,100 & 25,500 & 27,500 & 20,000 & 38,500 & 40,700 \\
\hline 12 & 31,000 & 29,800 & 11,000 & 6,500 & 10,500 & 20,200 & 25,100 & 25,100 & 37,700 & 22,000 & 39,000 & 40,700 \\
\hline 13 & 31,500 & 29,700 & 11,000 & 6,000 & 11,000 & 18,500 & 24,400 & 24,900 & 35,000 & 23,500 & 38,900 & 40,200 \\
\hline 14 & 32,100 & 29,000 & 10,000 & 6,500 & 11,000 & 17,000 & 26,600 & 24,500 & 34,400 & 23,800 & 39,000 & 39,400 \\
\hline 15 & 32,200 & 27,500 & 9,000 & 7,000 & 11,000 & 17,000 & 29,900 & 23,400 & 32,000 & 23,800 & 38,800 & 39,000 \\
\hline 16 & 31,900 & 25,500 & 7,500 & 7,500 & 11,500 & 22,000 & 30,400 & 23,100 & 31,100 & 23,800 & 38,400 & 38,600 \\
\hline 17 & 31,500 & 23,900 & 5,800 & 8,500 & 11,500 & 17,000 & 29,300 & 22,900 & 27,500 & 25,400 & 39,200 & 38,600 \\
\hline 18 & 30,900 & 22,500 & 5,800 & 9,000 & 11,500 & 15,000 & 27,600 & 23,000 & 23,600 & 27,900 & 39,400 & 38,700 \\
\hline 19 & 30,700 & 21,700 & 5,800 & 9,500 & 11,500 & 14,400 & 25,900 & 23,200 & 19,900 & 28,600 & 38,800 & 39,000 \\
\hline 20 & 31,300 & 21,200 & 5,800 & 10,000 & 11,500 & 14,800 & 25,400 & 23,400 & 17,200 & 28,600 & 38,200 & 39,300 \\
\hline 21 & 32,200 & 20,200 & 5,500 & 10,000 & 11,500 & 17,900 & 27,400 & 23,200 & 15,400 & 29,500 & 39,200 & 39,200 \\
\hline 22 & 33,600 & 19,100 & 5,000 & 9,500 & 13,000 & 22,200 & 28,600 & 23,200 & 14,600 & 32,100 & 39,600 & 39,000 \\
\hline 23 & 33,900 & 18,200 & 5,000 & 8,500 & 17,000 & 24,000 & 28,900 & 23,100 & 14,200 & 34,400 & 40,700 & 39,000 \\
\hline 24 & 33,000 & 17,700 & 5,000 & 8,500 & 16,700 & 25,600 & 27,200 & 23,300 & 13,900 & 34,800 & 40,000 & 38,300 \\
\hline 25 & 32,700 & 17,200 & 5,000 & 8,600 & 14,900 & 29,200 & 27,600 & 23,200 & 13,400 & 34,500 & 39,600 & 38,600 \\
\hline 26 & 32,400 & 17,100 & 5,000 & 8,700 & 15,000 & 27,800 & 27,600 & 23,800 & 13,200 & 34,000 & 38,300 & 38,800 \\
\hline 27 & 32,700 & 17,200 & 5,000 & 8,800 & 13,900 & 26,200 & 27,400 & 24,600 & 13,200 & 34,900 & 38,200 & 38,800 \\
\hline 28 & 33,400 & 17,300 & 6,500 & 8,900 & 13,600 & 22,100 & 27,400 & 26,200 & 13,100 & 35,500 & 38,200 & 38,800 \\
\hline 29 & 35,200 & 17,300 & 7,500 & 9,000 & -- & 20,300 & 28,100 & 26,200 & 13,000 & 35,600 & 38,300 & 38,000 \\
\hline 30 & 45,500 & 17,000 & 7,500 & 9,200 & -- & 19,000 & 28,100 & 26,300 & 12,400 & 36,300 & 38,300 & 38,300 \\
\hline 31 & 44,100 & --- & 8,000 & 9,500 & -- & 17,600 & --- & 27,200 & -.. & 38,500 & 38,600 & - \\
\hline TOTAL & $1,012,800$ & 771,900 & 297,700 & 260,800 & 324,600 & 593,200 & 692,600 & 784,800 & 705,900 & 767,700 & $1,206,300$ & $1,174,300$ \\
\hline MEAN & 32,670 & 25,730 & 9,603 & 8,413 & 11,590 & 19,140 & 23,090 & 25,320 & 23,530 & 24,760 & 38,910 & 39,140 \\
\hline MAX & 45,500 & 40,800 & 17,000 & 10,000 & 17,000 & 29,200 & 30,400 & 28,800 & 37,700 & 38,500 & 40,700 & 40,700 \\
\hline MIN & 30,400 & 17,000 & 5,000 & 6,000 & 9,700 & 10,000 & 12,600 & 22,900 & 12,400 & 12,600 & 38,200 & 38,000 \\
\hline AC-FT & $2,009,000$ & $1,531,000$ & 590,500 & 517,300 & 643,800 & $1,177,000$ & $1,374,000$ & $1,557,000$ & $1,400,000$ & $1,523,000$ & $2,393,000$ & $2,329,000$ \\
\hline
\end{tabular}


Table 17. Ponds, stock ponds, and dugouts on the Lower Brule Indian Reservation

[Where a pond forms behind a dam, the location given is that of the dam. A " $p$ " indicates the dam is located on a divide between quadrants and an " $\&$ " indicates the pond or dam is located in both quadrants. Those sites visited in February 1993 include a date of visitation. All other ponds listed were shown on aerial photographs taken in August and September 1991. Estimated size: a, less than 1 acre; b, equal to or greater than 1 acre but less than or equal to 5 acres; c, greater than 5 acres but less than or equal to 20 acres; d, greater than 20 acres. N, not visited; NA, not accessible at time of attempted visit; --, no data or not applicable]

\begin{tabular}{|c|c|c|c|c|c|c|}
\hline & Location & $\begin{array}{l}\text { Estimated } \\
\text { size }\end{array}$ & $\begin{array}{l}\text { Date of } \\
\text { visit }\end{array}$ & Condition & $\begin{array}{l}\text { Water-quality } \\
\text { sample }\end{array}$ & Comments \\
\hline \multirow[t]{4}{*}{ 105-71- } & $5 \mathrm{CBA}$ & $a$ & $\mathrm{~N}$ & -- & -- & -- \\
\hline & 6BDBD & $\mathrm{b}$ & $\mathbf{N}$ & -- & -- & -- \\
\hline & $6 \mathrm{CBAB} / \mathrm{C}$ & b & $\mathrm{N}$ & -- & -- & -- \\
\hline & 7BBCA & $\mathrm{b}$ & $\mathrm{N}$ & -- & -- & -- \\
\hline \multirow[t]{23}{*}{$105-72-$} & 1AABD & $\mathrm{b}$ & $\mathbf{N}$ & -- & -- & -- \\
\hline & $1 \mathrm{BBBC}$ & a & $\mathrm{N}$ & -- & -- & -- \\
\hline & $1 \mathrm{BCCD}$ & a & $\mathbf{N}$ & -- & -- & -- \\
\hline & 2BAAA & $\mathrm{a}$ & $\mathbf{N}$ & -- & -- & Dugout. \\
\hline & $2 \mathrm{CCBA}$ & b & $\mathrm{N}$ & -- & -- & -- \\
\hline & 3ADCA & $\mathrm{b}$ & $\mathrm{N}$ & -- & -- & -- \\
\hline & $3 \mathrm{CDB}$ & b & $\mathrm{N}$ & -- & -- & -- \\
\hline & $4 \mathrm{BBBC}$ & $\mathrm{a}$ & $\mathrm{N}$ & -- & -- & Dugout. \\
\hline & 4CCDD & b & $\mathbf{N}$ & -- & -- & -- \\
\hline & 4DAAA & b & $\mathrm{N}$ & -- & -- & -- \\
\hline & 5BADA/D & b & $\mathbf{N}$ & -- & -- & -- \\
\hline & $5 \mathrm{CDDD}$ & $\mathrm{a}$ & $\mathrm{N}$ & -- & -- & -- \\
\hline & 5DAA/D & c & $\mathrm{N}$ & -- & -- &.- \\
\hline & $6 \mathrm{AABC}$ & $\mathrm{a}$ & $\mathrm{N}$ & -- & -- & Dugout. \\
\hline & $6 \mathrm{CCCA}$ & $\mathrm{a}$ & $\mathrm{N}$ & -- & -- & -- \\
\hline & 6CCDD & b & $\mathrm{N}$ & -- & -- & -- \\
\hline & 6DDAA & $\mathrm{a}$ & $\mathrm{N}$ & -- & -- & -- \\
\hline & 7AABA & a & $\mathbf{N}$ & - & -- & Dugout. \\
\hline & 8ABCA & $\mathrm{b}$ & 2- $9-93$ & Poor & No & Dry. \\
\hline & 9ACCC & c & $\mathrm{N}$ & -- & -- & $-\cdot$ \\
\hline & 9BADC & b & $\mathrm{N}$ & -- & -- & -- \\
\hline & $10 \mathrm{AABC}$ & b & $N$ & -- & -- & -- \\
\hline & 10BCBB & b & $\mathrm{N}$ & -- & -- & -- \\
\hline \multirow[t]{11}{*}{ 106-71- } & $6 \mathrm{BCAB}$ & b & $\mathrm{N}$ & -- & -- & -- \\
\hline & $6 \mathrm{CBA}$ & b & $\mathbf{N}$ & -. & -- &.- \\
\hline & $6 \mathrm{CBBC}$ & a & $\mathrm{N}$ & - & -- & -- \\
\hline & $6 \mathrm{CCC}$ & $\mathrm{a}$ & $\mathrm{N}$ & -- & -- & Dam breached. \\
\hline & $7 \mathrm{BAA}$ & $\mathrm{a}$ & $\mathbf{N}$ & -- & -- & -- \\
\hline & 19BCAA & b & $\mathbf{N}$ & -- & -- & -- \\
\hline & $20 \mathrm{CBDB} / \mathrm{C}$ & $\mathrm{b}$ & $\mathbf{N}$ & -- & -- & Dam possibly breached. \\
\hline & 21CCAA & $\mathrm{a}$ & $\mathrm{N}$ & -- & -- & -- \\
\hline & 29BDAB/BA & $\mathrm{a}$ & $\mathrm{N}$ & -- & -- & -- \\
\hline & 29CADA & a & $\mathrm{N}$ & -- & -- & Dam possibly breached. \\
\hline & 30BDC & b & $\mathbf{N}$ & -- & -- & -- \\
\hline
\end{tabular}


Table 17. Ponds, stock ponds, and dugouts on the Lower Brule Indian Reservation—Continued

\begin{tabular}{|c|c|c|c|c|c|}
\hline Location & $\begin{array}{l}\text { Estimated } \\
\text { size }\end{array}$ & $\begin{array}{l}\text { Date of } \\
\text { visit }\end{array}$ & Condition & $\begin{array}{l}\text { Water-quality } \\
\text { sample }\end{array}$ & Comments \\
\hline 106-71-30CADB & $\mathbf{a}$ & $\mathbf{N}$ & -- & -- & -- \\
\hline 30DAAB & $\mathbf{b}$ & $\mathbf{N}$ & - & -- & -- \\
\hline $30 \mathrm{DBB}$ & $\mathbf{c}$ & $\mathbf{N}$ & -- & -- & -- \\
\hline 31BACB & $\mathbf{a}$ & $\mathbf{N}$ & -- & -- & Dam breached. \\
\hline 31CADA/D & $\mathbf{b}$ & $\mathbf{N}$ & -- & -- & Dam breached. \\
\hline 106-72- 1CDDB & b & $2-26-93$ & Poor & No & Dry; dam breached. \\
\hline 2BACD & a & $\mathbf{N}$ & -- & - & -- \\
\hline 2DAAA 1 & $\mathbf{a}$ & $\mathbf{N}$ & $\cdots$ & - & $\begin{array}{l}\text { Possibly not a present or former stock } \\
\text { pond. }\end{array}$ \\
\hline 2DAAA2 & $\mathbf{a}$ & $\mathbf{N}$ & -- & -- & $\begin{array}{l}\text { Possibly not a present or former stock } \\
\text { pond. }\end{array}$ \\
\hline 2DDBC & b & NA & -- & -- &.- \\
\hline 3BABB & a & $\mathbf{N}$ & -- &.- & -- \\
\hline 3CAAD & a & NA & -- & -- & -- \\
\hline $4 \mathrm{BCBC}$ & a & 2- $9-93$ & Above average & Yes & Spring fed. \\
\hline 7ACDB & a & $\mathbf{N}$ & -- & -. & -- \\
\hline 8AAAC & a & $\mathbf{N}$ & -- & -- & -- \\
\hline 8AABB & a & $\mathbf{N}$ & -- & -- & Dam possibly breached. \\
\hline 8CBDB & a & $\mathbf{N}$ & - & -- & -- \\
\hline 9ACBD & b & $\mathbf{N}$ & -- & -- & - \\
\hline 9BCA/D & b & $\mathbf{N}$ & -- & -- & -- \\
\hline 9DBA/B & $\mathbf{b}$ & $2-26-93$ & Poor & No & Dry; overgrown with vegetation. \\
\hline 10ADDC & $\mathbf{a}$ & $\mathbf{N}$ & -- & -- & -- \\
\hline 14DCBB & a & $\mathbf{N}$ & -- & -- & -- \\
\hline 15ABAD & a & $\mathbf{N}$ & -- & -- & -- \\
\hline 15DBDD & b & $\mathbf{N}$ & -- & -- & -- \\
\hline 16ADCC & a & $2-26-93$ & Above average & No & Frozen to bottom. \\
\hline 16BAAB & b & $\mathrm{N}$ & -- & -- & -- \\
\hline $17 \mathrm{CCAB}$ & a & $\mathbf{N}$ & -- & -- & -- \\
\hline 17CDDC & b & $2-26-93$ & Above average & No & Frozen to bottom \\
\hline 17CDDD & $\mathbf{a}$ & $\mathbf{N}$ & -- & -- & -- \\
\hline 18ACDA/D & a & $\mathbf{N}$ & -- & -- & -- \\
\hline 19ACBB/C & b & $\mathbf{N}$ & -- & -- & -- \\
\hline 19ACDC & $\mathrm{a}$ & $\mathbf{N}$ & -- & -- & -- \\
\hline 19BABC & $\mathbf{a}$ & $\mathrm{N}$ &.- & -- & -- \\
\hline 19CCAD & b & $\mathbf{N}$ & -- & -- & -- \\
\hline 19CDBB & b & $\mathbf{N}$ & -- & -- & -- \\
\hline 19DCAD & $\mathbf{a}$ & $\mathbf{N}$ & -- & -- & Dugout. \\
\hline 20CDAB/BA & b & $\mathrm{N}$ & -- & -- & -- \\
\hline 20DCBC & a & $\mathbf{N}$ & -- & -- & Dugout. \\
\hline $21 \mathrm{CACD}$ & a & $\mathbf{N}$ & - & -- & -- \\
\hline $21 \mathrm{CCCA}$ & a & $\mathbf{N}$ & -- & -- & -- \\
\hline 21DDCC/D & b & $\mathbf{N}$ & -- & 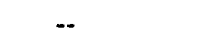 & -- \\
\hline
\end{tabular}


Table 17. Ponds, stock ponds, and dugouts on the Lower Brule Indian Reservation-Continued

\begin{tabular}{|c|c|c|c|c|c|}
\hline Location & $\begin{array}{l}\text { Estimated } \\
\text { size }\end{array}$ & $\begin{array}{c}\text { Date of } \\
\text { visit }\end{array}$ & Condition & $\begin{array}{l}\text { Water-quality } \\
\text { sample }\end{array}$ & Comments \\
\hline 106-72- 22ADC & b & $\mathbf{N}$ & -- & -- & -- \\
\hline 22BACB & b & $\mathrm{N}$ & -- & -- & -- \\
\hline 23ADDA & a & $\mathrm{N}$ & -- & -- & -- \\
\hline $23 \mathrm{BCA}$ & a & $\mathbf{N}$ & -- & -- & -- \\
\hline $24 \mathrm{ABBB}$ & b & $\mathbf{N}$ & -- & -- & -- \\
\hline 25ADCD & a & $\mathbf{N}$ & -. & -- & -- \\
\hline 25DACD & b & $\mathbf{N}$ & -- & -- & -- \\
\hline 26AAAA & b & $\mathrm{N}$ & -- & -- & -- \\
\hline 26BBBB & a & $\mathbf{N}$ & -- & - & -- \\
\hline 26BBBD & c & $\mathbf{N}$ & -- & -- & Dam breached. \\
\hline 26CBDA & a & $\mathbf{N}$ & -- & -- & Dugout. \\
\hline 26DBCA & $\mathbf{a}$ & $2-26-93$ & Poor & No & Dry; overgrown with vegetation. \\
\hline 26DBDD & a & $\mathrm{N}$ & -- & -- & -- \\
\hline $27 \mathrm{AAAB}$ & a & $\mathbf{N}$ & -- & -- & -- \\
\hline 27DBCD & $\mathbf{a}$ & $\mathbf{N}$ & -- & -- & -- \\
\hline 28BACD1 & a & $\mathbf{N}$ & -- & -- & -- \\
\hline 28BACD2 & $\mathbf{a}$ & $\mathbf{N}$ & -- & -- & -- \\
\hline 28DBA/D & b & $2-26-93$ & Poor & No & Dry; overgrown with vegetation. \\
\hline 28DDDC & b & $\mathbf{N}$ & -- & -. & -- \\
\hline 29BACD & $\mathbf{a}$ & $\mathbf{N}$ & -- & -- & Dugout. \\
\hline 29DABB & a & $\mathbf{N}$ & -- & -- & Dugout. \\
\hline $30 \mathrm{ABAC}$ & a & $\mathbf{N}$ & -- & -- & Dugout. \\
\hline $30 \mathrm{ABBD}$ & b & $\mathbf{N}$ & -- & -- & -- \\
\hline $\begin{array}{l}\text { 30BAAA \& } \\
\text { ABBB }\end{array}$ & c & $\mathbf{N}$ & -- & -- & -- \\
\hline 30BAAC & a & $\mathbf{N}$ & -- & -- & -- \\
\hline $31 \mathrm{ACAA}$ & b & $\mathbf{N}$ & -- & -- & -- \\
\hline $31 \mathrm{BBCC}$ & a & $\mathrm{N}$ & -- & -- & Dugout. \\
\hline 31DADC & b & $\mathbf{N}$ & -- & -- & -- \\
\hline 32AADB & b & $\mathbf{N}$ & -- & -- & Dugout. \\
\hline $32 \mathrm{BABD}$ & $\mathrm{a}$ & $\mathbf{N}$ & -- & -- & Dugout. \\
\hline $32 \mathrm{CDBA}$ & a & $\mathrm{N}$ & -- & -- & Dugout. \\
\hline $32 \mathrm{CDCA}$ & b & $\mathrm{N}$ & -- & -- & -- \\
\hline 32DDAC & $\mathrm{a}$ & $\mathbf{N}$ & -- & -- & Dugout. \\
\hline 33BBD & $\mathrm{a}$ & $\mathbf{N}$ & -- & -- & Dugout. \\
\hline $33 \mathrm{CDDD}$ & a & $\mathbf{N}$ & -- & -- & Dugout. \\
\hline 33DDDD & a & $\mathbf{N}$ & -- & -- & Dam breached. \\
\hline $34 \mathrm{ABAB}$ & a & $\mathbf{N}$ & -- & -- & -- \\
\hline $34 \mathrm{BCC}$ & d & $2-26-93$ & Above average & No & $\begin{array}{l}\text { Large; overgrown with vegetation; frozen } \\
\text { to bottom. }\end{array}$ \\
\hline 34BD & -- & $2-26-93$ & Poor & No & Dry; overgrown with vegetation. \\
\hline $34 \mathrm{CCDB}$ & b & $\mathrm{N}$ & - & -- & -- \\
\hline $35 \mathrm{ABBC}$ & a & $\mathrm{N}$ & -- & -- & Dam breached. \\
\hline
\end{tabular}


Table 17. Ponds, stock ponds, and dugouts on the Lower Brule Indian Reservation-Continued

\begin{tabular}{|c|c|c|c|c|c|c|}
\hline & Location & $\begin{array}{l}\text { Estimated } \\
\text { size }\end{array}$ & $\begin{array}{l}\text { Date of } \\
\text { visit }\end{array}$ & Condition & $\begin{array}{c}\text { Water-quality } \\
\text { sample }\end{array}$ & Comments \\
\hline $106-72-$ & $36 \mathrm{ACD}$ & $\bar{a}$ & $\mathbf{N}$ & -- & -- & -- \\
\hline & 36DABD & c & $\mathbf{N}$ & -- & -- & -- \\
\hline \multirow[t]{10}{*}{ 106-73- } & 1AAAA & a & $\mathbf{N}$ & -- & -- & Dam breached. \\
\hline & 1BDBB & $\mathbf{a}$ & $\mathbf{N}$ & -- & -- & -- \\
\hline & 2BBAA & b & $\mathbf{N}$ & -- & -- & -- \\
\hline & 3ACAC & b & $\mathbf{N}$ & -- & -- & -- \\
\hline & 4AADD & $\mathbf{a}$ & $\mathbf{N}$ & -- & -- & Dam breached. \\
\hline & 4ABDC & a & $\mathbf{N}$ & -- & -- & Dugout. \\
\hline & 4BDDC & c & $\mathbf{N}$ & -- & -- & -- \\
\hline & 6ADAC & b & $\mathbf{N}$ & -- & -- & Dam breached. \\
\hline & $6 \mathrm{BABC}$ & a & $\mathbf{N}$ & -- & -- & Dry; dam possibly breached. \\
\hline & 6BDAA & a & $\mathbf{N}$ & -- & -- & Dugout, dry. \\
\hline \multirow[t]{5}{*}{$106-74$} & 1BAAB & b & $\mathbf{N}$ & -- & -- & -- \\
\hline & 2AAC/D & a & $\mathbf{N}$ & -- & -- & -- \\
\hline & $3 \mathrm{BCBD}$ & b & $\mathbf{N}$ & -- & -- & -- \\
\hline & $4 \mathrm{ABCB}$ & b & $\mathbf{N}$ & -- & -- & -- \\
\hline & 4ABCA/DC & $\mathrm{b}$ & $\mathbf{N}$ & -- & -- & Dam breached. \\
\hline \multirow[t]{9}{*}{ 106-75- } & 1AAAA & a & $\mathbf{N}$ & -- & -- & Possibly dry. \\
\hline & $1 \mathrm{BBCD}$ & $\mathrm{b}$ & $\mathrm{N}$ & -- & -- & -- \\
\hline & 2BDDA/D & $\mathbf{a}$ & $\mathbf{N}$ & -- & -- & Dugout. \\
\hline & $3 \mathrm{ADBB} / \mathrm{C}$ & $\mathrm{b}$ & $\mathbf{N}$ & -- & - & -- \\
\hline & 3BACA/B & a & $\mathbf{N}$ & -- & -- & -- \\
\hline & $3 \mathrm{BBAB} / \mathrm{C}$ & $\mathrm{b}$ & $\mathrm{N}$ & -- & -- &.- \\
\hline & $4 \mathrm{ACC}$ & d & $\mathbf{N}$ & -- & -- & -- \\
\hline & $4 \mathrm{ACDD}$ & $\mathbf{a}$ & $\mathbf{N}$ & -- & -- & Possibly a stock pond. \\
\hline & 4BBCA & a & $\mathbf{N}$ & -- & -- & -- \\
\hline \multirow[t]{2}{*}{ 106-76- } & $3 \mathrm{BDCC}$ & a & $\mathbf{N}$ & -- & -- & -- \\
\hline & 6AAAA & $\mathbf{a}$ & $\mathbf{N}$ & - & -- & -- \\
\hline \multirow[t]{6}{*}{$106-77-$} & 1BCAC/D & b & $\mathbf{N}$ & - & -- & -- \\
\hline & $1 \mathrm{BCCC}$ & a & $\mathbf{N}$ & - & -- & -- \\
\hline & $2 \mathrm{ADCC}$ & $\mathbf{a}$ & $\mathbf{N}$ & -- & -- & Dugout. \\
\hline & 2BBAD & $\mathbf{a}$ & $\mathbf{N}$ & -- & - & Dugout. \\
\hline & 2BBBB & $\mathrm{b}$ & $\mathbf{N}$ & -- & -- & -- \\
\hline & $3 \mathrm{ABDB}$ & $\mathbf{a}$ & $\mathbf{N}$ & -- & -- & Possibly a dugout. \\
\hline \multirow[t]{4}{*}{$107-72-$} & 32AADD & b & $\mathrm{N}$ & -- & -- & -- \\
\hline & $32 \mathrm{CDBC}$ & $\mathbf{a}$ & $\mathbf{N}$ & -- & -- & -- \\
\hline & 33DCBA/B & b & $\mathbf{N}$ & -- & -- & -- \\
\hline & $\begin{array}{l}\text { 35DBDD \& } \\
\text { DACC }\end{array}$ & $\mathrm{a}$ & $\mathbf{N}$ & -- & -- & Dam breached. \\
\hline \multirow[t]{4}{*}{ 107-73- } & 4DBA & b & $\mathbf{N}$ & -- & -- & -- \\
\hline & 4DCDD & a & $\mathbf{N}$ & -- & - & Dugout. \\
\hline & 4DDCB & $\mathrm{a}$ & $\mathbf{N}$ & -- & - & Possibly a slough. \\
\hline & $7 \mathrm{DBCA}$ & a & $\mathrm{N}$ & -- & -- & -- \\
\hline
\end{tabular}


Table 17. Ponds, stock ponds, and dugouts on the Lower Brule Indian Reservation-Continued

\begin{tabular}{|c|c|c|c|c|c|c|}
\hline & Location & $\begin{array}{l}\text { Estimated } \\
\text { size }\end{array}$ & $\begin{array}{l}\text { Date of } \\
\text { visit }\end{array}$ & Condition & $\begin{array}{l}\text { Water-quality } \\
\text { sample }\end{array}$ & Comments \\
\hline \multirow[t]{39}{*}{ 107-73- } & $8 \mathrm{ADCC}$ & $\mathrm{a}$ & NA & -- & -- & -- \\
\hline & $8 \mathrm{BCAC}$ & a & $\mathrm{N}$ & -- & -- & -- \\
\hline & 9DBDC & $b$ & $\mathrm{~N}$ & -- & -- &.- \\
\hline & $10 \mathrm{CBCB}$ & $\mathrm{a}$ & $\mathrm{N}$ & -- & -- & -- \\
\hline & $15 \mathrm{ACCD}$ & b & $\mathrm{N}$ & -- & -- & Sewage pond. \\
\hline & $\begin{array}{l}\text { 15ACDC \& } \\
\text { DBAB }\end{array}$ & b & $\mathrm{N}$ & -- & -- & Sewage pond. \\
\hline & $15 \mathrm{ACDD}$ & $\mathrm{a}$ & $\mathrm{N}$ & -- & -- & Sewage pond. \\
\hline & 15DBAA & b & $\mathrm{N}$ & -- & - & Sewage pond. \\
\hline & $15 \mathrm{DBB} / \mathrm{C} / \mathrm{D}$ & $\mathrm{b}$ & $\mathrm{N}$ & -- & -- & Sewage pond. \\
\hline & $15 \mathrm{CBC}$ & b & $\mathrm{N}$ & -- & -- & -- \\
\hline & $15 \mathrm{CCAD}$ & a & $\mathrm{N}$ & -- & -- & -- \\
\hline & $16 \mathrm{ACDC} / \mathrm{D}$ & b & $2-9-93$ & -- & Yes & Sewage lagoon. \\
\hline & $16 \mathrm{CBBD}$ & a & $2-23-93$ & Poor & No & $\begin{array}{l}\text { Dry; overgrown with vegetation; dam } \\
\text { breached. }\end{array}$ \\
\hline & 17AABB & a & $\mathrm{N}$ & -- & -- & -- \\
\hline & 17BCAC & $\mathrm{b}$ & $\mathrm{N}$ & -- & -- & Possibly a slough. \\
\hline & $17 \mathrm{BCD}$ & b & $\mathrm{N}$ & -- & -- & Possibly a slough. \\
\hline & 17CDAB & a & $\mathrm{N}$ & -- & -- & -- \\
\hline & 17DDDD & a & $\mathrm{N}$ & -- & -- & -- \\
\hline & 18AACC & b & $\mathbf{N}$ & -- & -- & Possibly a sewage pond. \\
\hline & 18ABDA & a & $\mathrm{N}$ & -- & -- & Possibly a sewage pond. \\
\hline & 18ABDD & a & $\mathbf{N}$ & -- & -- & Possibly a sewage pond. \\
\hline & 18BAAD & a & $\mathbf{N}$ & -- & -- & -- \\
\hline & 18DCAA & a & $\mathrm{N}$ & -- & -- & -- \\
\hline & 18DDCD & a & $\mathrm{N}$ & -- & -- & -- \\
\hline & 19AAAA & a & $2-23-93$ & Below average & No & $\begin{array}{l}\text { Overgrown with vegetation; frozen to } \\
\text { bottom. }\end{array}$ \\
\hline & $19 \mathrm{ACC}$ & b & $\mathrm{N}$ & -- & -- & -- \\
\hline & 19ADAA/B & a & $2-23-93$ & Poor & No & Dugout dry. \\
\hline & 19BCAC & b & 2-23-93 & Below average & Yes & -- \\
\hline & 19BDC & b & $2-23-93$ & Poor & No & Dry; dam breached. \\
\hline & 19CBBA & b & $\mathrm{N}$ & -- & -- & -- \\
\hline & 19DBAB & a & $\mathrm{N}$ & -- & -- & -- \\
\hline & 19DCAB & a & $\mathrm{N}$ & -- & -- & Dugout. \\
\hline & 20BBDD & b & $2-23-93$ & Poor & No & Dry. \\
\hline & 20DCAB & b & $\mathrm{N}$ & -- & -- & -- \\
\hline & 20DDBD & $\mathbf{a}$ & $\mathrm{N}$ & -- & -- & -- \\
\hline & 21BBAC & $\mathbf{a}$ & $\mathrm{N}$ & -- & -- & -- \\
\hline & $22 \mathrm{ABAD}$ & $\mathbf{a}$ & $\mathrm{N}$ & -- & -- & -- \\
\hline & $22 \mathrm{ABCA}$ & a & $\mathrm{N}$ & -- & -- & -- \\
\hline & $\begin{array}{c}\text { 23BCBD \& } \\
\text { BCAC } 1\end{array}$ & a & $\mathrm{N}$ & -- & -- & -- \\
\hline
\end{tabular}


Table 17. Ponds, stock ponds, and dugouts on the Lower Brule Indian Reservation-Continued

\begin{tabular}{|c|c|c|c|c|c|c|}
\hline & Location & $\begin{array}{l}\text { Estimated } \\
\text { size }\end{array}$ & $\begin{array}{c}\text { Date of } \\
\text { visit }\end{array}$ & Condition & $\begin{array}{c}\text { Water-quality } \\
\text { sample }\end{array}$ & Comments \\
\hline \multirow[t]{19}{*}{ 107-73- } & $\begin{array}{l}\text { 23BCBD \& } \\
\text { BCAC2 }\end{array}$ & $\mathbf{a}$ & $\mathbf{N}$ & -- & -- & -- \\
\hline & 23CDCA/D & b & $\mathrm{N}$ & -- & -- & -- \\
\hline & $24 \mathrm{CCDB}$ & b & $2-26-93$ & Poor & No & Dry; overgrown with vegetation. \\
\hline & $24 C C D D$ & a & $\mathbf{N}$ & -- & - & -- \\
\hline & 24CDCA & a & $\mathbf{N}$ & -. & -- & -- \\
\hline & $25 \mathrm{CCAA}$ & a & $\mathbf{N}$ & - & -- & - \\
\hline & 29DBAA & b & $\mathrm{N}$ & - & - & -- \\
\hline & $30 \mathrm{CBCB}$ & a & $\mathbf{N}$ & - & -- & -- \\
\hline & $30 \mathrm{CCAC}$ & b & $2-8-93$ & Poor & Yes & $\begin{array}{l}\text { Dam center slumped; overgrown with } \\
\text { trees. }\end{array}$ \\
\hline & 31AACA & a & $\mathbf{N}$ & - & -- & -- \\
\hline & 32BCCC/D & b & $\mathbf{N}$ & - & -- & -- \\
\hline & $33 \mathrm{AABB}$ & b & $\mathbf{N}$ & -- & -- & -- \\
\hline & $33 \mathrm{ACBC}$ & a & $\mathbf{N}$ & -- & - & Possibly dry. \\
\hline & 33BBD & b & $\mathbf{N}$ & -- & -- & -- \\
\hline & 33DABA/B & $\mathrm{c}$ & $\mathrm{N}$ & -- & -- & -- \\
\hline & $34 \mathrm{CACC}$ & a & $\mathrm{N}$ & -- & -- & -- \\
\hline & 34CBDA & a & N & -- & -- & -- \\
\hline & $35 \mathrm{CDDC}$ & b & $\mathbf{N}$ & -- & -- & -- \\
\hline & $36 \mathrm{BCC}$ & $\mathrm{b}$ & $N$ & -- & -- & - \\
\hline \multirow[t]{21}{*}{$107-74-$} & - 3DCDD & a & $\mathbf{N}$ & -- & -- & -- \\
\hline & 3DDBA & a & $\mathrm{N}$ & -- & -- & Dam breached. \\
\hline & 3DDB & $\mathrm{b}$ & N & -- & - & Dam breached. \\
\hline & 6BDA & c & $\mathbf{N}$ & -- & -- & -- \\
\hline & 7BDCD & b & N & -- & - & -- \\
\hline & $\begin{array}{l}\text { 7CABC/CA/ } \\
\mathrm{CB}\end{array}$ & b & $\mathrm{N}$ & -- & -- & -- \\
\hline & 7DDAA & a & $\mathrm{N}$ & -- & -- & -- \\
\hline & 8CAAD & a & $\mathbf{N}$ & -- & -- & Dugout. \\
\hline & 9DCAB & a & $\mathbf{N}$ & -- & -- & Dugout. \\
\hline & 9DCAA & a & $\mathrm{N}$ & -. & -- & Dugout. \\
\hline & 10BAAB & a & $\mathrm{N}$ & -- & -- & -- \\
\hline & 11BBAA & a & $\mathbf{N}$ & -- & -- & -- \\
\hline & $11 \mathrm{CBCB}$ & a & $\mathbf{N}$ & - & -- & -- \\
\hline & 12ACBA & a & $\mathbf{N}$ & -- & -- & -- \\
\hline & 12BCBB & a & N & -- & -- & -- \\
\hline & 12DDCB & a & $\mathrm{N}$ & -- & -- & Possibly a slough. \\
\hline & $13 \mathrm{ABBD}$ & a & $\mathbf{N}$ & -- & -- & Dugout. \\
\hline & $14 \mathrm{ADC}$ & c & $\mathbf{N}$ & -- & -- & Dam breached. \\
\hline & 15DADC & b & $\mathrm{N}$ & -- & -- & -- \\
\hline & $16 \mathrm{ACBC}$ & a & $\mathbf{N}$ & -- & -- & -- \\
\hline & $16 \mathrm{CCCA}$ & a & $\mathrm{N}$ & -- & -- & -- \\
\hline
\end{tabular}


Table 17. Ponds, stock ponds, and dugouts on the Lower Brule Indian Reservation-Continued

\begin{tabular}{|c|c|c|c|c|c|c|}
\hline & Location & $\begin{array}{l}\text { Estimated } \\
\text { size }\end{array}$ & $\begin{array}{c}\text { Date of } \\
\text { visit }\end{array}$ & Condition & $\begin{array}{l}\text { Water-quality } \\
\text { sample }\end{array}$ & Comments \\
\hline \multirow[t]{29}{*}{$107-74-$} & 16DADB & $\mathrm{a}$ & $\mathbf{N}$ & -- & -- & -- \\
\hline & 18ACBD & b & $\mathrm{N}$ & -- & -- & -- \\
\hline & 19BBAA & $\mathbf{a}$ & $\mathrm{N}$ & -- & -- & -- \\
\hline & 22BADD & $\mathbf{a}$ & $\mathrm{N}$ & -- & -- & Dugout. \\
\hline & 22CAAC & a & $\mathbf{N}$ & -- & -- & -- \\
\hline & $22 \mathrm{CCBA}$ & a & $\mathbf{N}$ & -- & -- & - \\
\hline & 23ADCA & b & $\mathbf{N}$ & -- & -- & -- \\
\hline & 23CADD & $\mathbf{a}$ & $\mathrm{N}$ & -- & -- & -- \\
\hline & 23CDAD & c & $\mathrm{N}$ & -- & -- & -- \\
\hline & 24ABDB & b & $\mathbf{N}$ & -- & -- & Dam possibly breached. \\
\hline & 24CADB & b & $\mathrm{N}$ & -- & -- & Dam breached. \\
\hline & $\begin{array}{c}\text { 24CDDD \& } \\
\text { DCCC }\end{array}$ & c & $\mathrm{N}$ & -- & -- & -- \\
\hline & $25 \mathrm{CCB}$ & c & $\mathbf{N}$ & -- & -- & -- \\
\hline & 25DBAB & b & $\mathbf{N}$ & -- & -- & Dam breached. \\
\hline & 26BADD & c & $\mathbf{N}$ & -- & -- & -- \\
\hline & 27ABAA & a & $\mathrm{N}$ & -- & -- & -- \\
\hline & 27ADBC & a & $\mathrm{N}$ & -- & -- & -- \\
\hline & 27CADA/B & a & $\mathrm{N}$ & -- & -- & -- \\
\hline & 28DCBB & b & $\mathrm{N}$ & -- & -- & -- \\
\hline & 30ACCA & b & $\mathrm{N}$ & -- & -- & -- \\
\hline & 31BBAA & b & $2-8-93$ & Above average & Yes & Spring fed. \\
\hline & 31CAAC & b & $\mathrm{N}$ & -- & -- & -- \\
\hline & $32 \mathrm{ADBC}$ & a & $\mathrm{N}$ & -- & -- & -- \\
\hline & $33 \mathrm{ADCC}$ & b & $\mathrm{N}$ & -- & -- & -- \\
\hline & $34 \mathrm{CCAC}$ & b & $\mathrm{N}$ & -- & -- & -- \\
\hline & 34DDDC/D & b & $\mathrm{N}$ & -- & -- & -- \\
\hline & $35 \mathrm{ADBB}$ & b & $\mathrm{N}$ & -- &.- & -- \\
\hline & $35 \mathrm{CABD}$ & b & $\mathbf{N}$ & -- & -- & -- \\
\hline & 36AADC & b & $\mathrm{N}$ & -- & -- & -- \\
\hline \multirow[t]{11}{*}{$107-75-$} & 1BAAD & $\mathbf{a}$ & $\mathrm{N}$ & -. & -- & Dugout. \\
\hline & 2BACC & b & $\mathrm{N}$ & -- & -- & -- \\
\hline & $\begin{array}{c}\text { 2CDAA \& } \\
\text { CADD }\end{array}$ & b & NA & -- & -- & -- \\
\hline & 2DAAC & b & $\mathrm{N}$ & -- & -- & -- \\
\hline & 3BBBB & a & $2-24-93$ & Poor & No & Dry; overgrown with trees. \\
\hline & $3 \mathrm{CADA} / \mathrm{D}$ & b & $2-24-93$ & Above average & Yes & -- \\
\hline & 3DDCA & c & NA & -- & -- & -- \\
\hline & $4 \mathrm{BB}$ & -- & $2-24-93$ & Poor & No & Dry; overgrown with vegetation. \\
\hline & 4ADCA & a & $\mathrm{N}$ & -- & -- & Dam breached. \\
\hline & 4ADCC/D & $\mathrm{a}$ & $\mathrm{N}$ & -- & -- & -- \\
\hline & $4 \mathrm{CA}$ & a & $2-24-93$ & Below average & No & $\begin{array}{l}\text { Dry; overgrown with vegetation, } \\
\text { although dam appears to be in good } \\
\text { condition. }\end{array}$ \\
\hline
\end{tabular}


Table 17. Ponds, stock ponds, and dugouts on the Lower Brule Indian Reservation-Continued

\begin{tabular}{|c|c|c|c|c|c|c|}
\hline & Location & $\begin{array}{l}\text { Estimated } \\
\text { size }\end{array}$ & $\begin{array}{l}\text { Date of } \\
\text { visit }\end{array}$ & Condition & $\begin{array}{c}\text { Water-quality } \\
\text { sample }\end{array}$ & Comments \\
\hline $107-75$ & 4CBAA/B & $\mathrm{a}$ & $2-24-93$ & Below average & No & $\begin{array}{l}\text { Dry; overgrown with vegetation, } \\
\text { although dam appears to be in good } \\
\text { condition. }\end{array}$ \\
\hline & $5 \mathrm{ABCB}$ & a & $\mathrm{N}$ & -- & -- & -- \\
\hline & 5BBCD & b & $\mathbf{N}$ & -- & -- & -- \\
\hline & $5 \mathrm{DAB}$ & a & $\mathbf{N}$ & -- & -- & -- \\
\hline & 5DDAD & b & $\mathbf{N}$ & -- & -- & -- \\
\hline & 6AABA & b & $\mathbf{N}$ & -- & -- & -- \\
\hline & 6ACBB & b & $\mathbf{N}$ & -- & -- & -- \\
\hline & 6ADDB & b & $\mathbf{N}$ & -- & -- & -- \\
\hline & 6BCAC & b & $\mathbf{N}$ & -- & -- & -- \\
\hline & $\begin{array}{c}\text { 6DABC \& } \\
\text { DBAD }\end{array}$ & b & $\mathbf{N}$ & -- & -- & -- \\
\hline & 6DBDD & b & $\mathbf{N}$ & -- & -- & -- \\
\hline & 7AADC/D & c & $\mathrm{N}$ & -- & -- & Dam breached. \\
\hline & $7 \mathrm{DCCC} / \mathrm{D}$ & b & $2-11-93$ & Poor & No & Overgrown with vegetation. \\
\hline & $\begin{array}{c}\text { 8BBBA \& } \\
\text { BBAB }\end{array}$ & b & -- & -- & -- & Dam breached. \\
\hline & $8 \mathrm{BCDD}$ & c & $\mathbf{N}$ & -- & -- &.- \\
\hline & $8 \mathrm{CBB}$ & c & $\mathrm{N}$ & -- & -- & -- \\
\hline & 9ABAA/B & b & $\mathbf{N}$ & -- & -- & -- \\
\hline & 9BBCD/BC & a & $\mathbf{N}$ & -- & -- & Dam breached. \\
\hline & 9BBD & b & $\mathbf{N}$ & -- & -- & -- \\
\hline & $9 \mathrm{BCBA}$ & $\mathbf{a}$ & $\mathbf{N}$ & -- & -- & -- \\
\hline & 9CBAB & $\mathbf{a}$ & $\mathbf{N}$ & -. & -- & -- \\
\hline & 9DCCA/B & b & $\mathbf{N}$ & -- & -- & -- \\
\hline & 9DCCD & a & $\mathbf{N}$ & -- & -- & Dam breached. \\
\hline & $\begin{array}{c}\text { 10DAB \& } \\
\text { ADC }\end{array}$ & b & $2-24-93$ & -- & No & -- \\
\hline & $\begin{array}{r}11 \mathrm{BCC} \& \\
\mathrm{CBA} / \mathrm{B}\end{array}$ & b & -- & -- & -- & -- \\
\hline & $\begin{array}{c}\text { 11DDCA \& } \\
\text { DDDB }\end{array}$ & b & 2- 8-93 & Above average & Yes & -- \\
\hline & 12BADD & b & $\mathbf{N}$ & -- & -- & -- \\
\hline & $13 \mathrm{ABCD}$ & a & $\mathbf{N}$ & -- & -- & -- \\
\hline & 13BDDC & b & $\mathrm{N}$ & -- & -- & -- \\
\hline & 13DBCD & $\mathbf{a}$ & $\mathbf{N}$ & -- & -- & -- \\
\hline & $\begin{array}{l}\text { 14DDDD \& } \\
13 \mathrm{CCCC}\end{array}$ & c & $\mathbf{N}$ & -. & -- & -. \\
\hline & $15 \mathrm{ACBC}$ & b & $\mathbf{N}$ & -- & -- & -- \\
\hline & $15 \mathrm{CAAB}$ & a & $\mathrm{N}$ & -- & -- & Dam possibly breached. \\
\hline & 15CBCD/DC & b & $\mathbf{N}$ & -- & -- & -- \\
\hline & $16 \mathrm{AAB}$ & b & $\mathbf{N}$ & -- & -- & -- \\
\hline & $\begin{array}{c}\text { 16BDBB \& } \\
\text { BACC }\end{array}$ & b & $\mathbf{N}$ & -- & -- & -- \\
\hline
\end{tabular}


Table 17. Ponds, stock ponds, and dugouts on the Lower Brule Indian Reservation-Continued

\begin{tabular}{|c|c|c|c|c|c|}
\hline Location & $\begin{array}{l}\text { Estimated } \\
\text { size }\end{array}$ & $\begin{array}{l}\text { Date of } \\
\text { visit }\end{array}$ & Condition & $\begin{array}{l}\text { Water-quality } \\
\text { sample }\end{array}$ & Comments \\
\hline $\begin{array}{c}107-75-16 C C D B \& \\
\text { CCAC }\end{array}$ & $\mathrm{b}$ & $\mathrm{N}$ & -- & -- & -- \\
\hline $16 \mathrm{CDDA}$ & $\mathrm{b}$ & $\mathrm{N}$ & -- & -- & -- \\
\hline $17 \mathrm{ADBC}$ & b & NA & -- & -- & -- \\
\hline 17ADDB & b & NA & -- & -- & -- \\
\hline 17BDBB & $\mathbf{a}$ & $2-9-93$ & Poor & No & Dry. \\
\hline $17 \mathrm{CCAB}$ & a & $\mathrm{N}$ & -- & - & Dugout. \\
\hline $\begin{array}{c}\text { 18ADBB \& } \\
\text { ACAA }\end{array}$ & c & $\mathbf{N}$ & -- & -- & Dam breached. \\
\hline $\begin{array}{c}\text { 18BACB \& } \\
\text { BBDB }\end{array}$ & a & $\mathrm{N}$ & -- & -- & -- \\
\hline 19DAA & a & $\mathbf{N}$ & -- & -- & -- \\
\hline 20BCDA & c & $\mathrm{N}$ & -- & -- & -- \\
\hline $\begin{array}{l}\text { 21 ACAD \& } \\
\text { ADBC }\end{array}$ & c & $\mathrm{N}$ & -- & - & -- \\
\hline 21BDCB & a & $\mathrm{N}$ & -- & -- & -- \\
\hline $22 \mathrm{AABC} / \mathrm{D}$ & b & $\mathrm{N}$ & -- & -- & - \\
\hline 22CBAC & $\mathrm{b}$ & $\mathbf{N}$ & -- & -- & - \\
\hline 22DDB & b & $\mathrm{N}$ & -- & - & -- \\
\hline $\begin{array}{c}\text { 23ABDC \& } \\
\text { ACAB }\end{array}$ & b & $\mathrm{N}$ & -- & -- & -- \\
\hline $24 \mathrm{ACAB}$ & a & $\mathrm{N}$ & -- & -- & -- \\
\hline $24 \mathrm{ADCB}$ & a & $\mathrm{N}$ & -- & -- & -- \\
\hline $25 \mathrm{BCBB}$ & a & $\mathrm{N}$ & -- & - & -- \\
\hline 25DBAA/B & a & $\mathrm{N}$ & -- & -- & -- \\
\hline 26BBDC & b & $\mathrm{N}$ & -- & -- & -- \\
\hline $26 \mathrm{CBCC}$ & b & $\mathrm{N}$ & -- & -- & -- \\
\hline 27ACCB & b & $\mathrm{N}$ & -- & -- & -- \\
\hline $28 \mathrm{AAB}$ & a & $\mathrm{N}$ & -. & -- & -- \\
\hline 28BDBC & a & $\mathrm{N}$ & -- & -- & Dugout. \\
\hline $29 \mathrm{CBDC} / \mathrm{D}$ & b & $\mathrm{N}$ & -. & -- & -- \\
\hline 29CCAA & b & $\mathrm{N}$ & -- & -- & -- \\
\hline $29 \mathrm{CCDD}$ & $\mathbf{a}$ & $\mathbf{N}$ & -- & -. & -- \\
\hline $30 \mathrm{ADDA}$ & a & $\mathbf{N}$ & -- & - & -- \\
\hline 30BCD & c & $\mathrm{N}$ & -- & -- & -- \\
\hline $31 \mathrm{CDCD}$ & a & $\mathrm{N}$ & -- & -- & -- \\
\hline 31DBDD & a & $\mathrm{N}$ & -- & -- & -- \\
\hline 32BDBA & $\mathbf{a}$ & $\mathrm{N}$ & -- & -- & -- \\
\hline $32 \mathrm{CBDA}$ & $\mathrm{b}$ & $\mathbf{N}$ & - & -- & -- \\
\hline 32DCDA & $\mathrm{b}$ & $\mathbf{N}$ & - & -- & -- \\
\hline 33AADA & $\mathrm{b}$ & $\mathrm{N}$ & -- & -- & -- \\
\hline 33DADD & b & $\mathbf{N}$ & -- & - & Dam breached. \\
\hline 33DDAA & $\mathrm{b}$ & $\mathrm{N}$ & -- & -- & -- \\
\hline $34 \mathrm{CDA} / \mathrm{B}$ & a & $\mathrm{N}$ & -- & -- & Dam breached. \\
\hline
\end{tabular}


Table 17. Ponds, stock ponds, and dugouts on the Lower Brule Indian Reservation-Continued

\begin{tabular}{|c|c|c|c|c|c|c|}
\hline & Location & $\begin{array}{l}\text { Estimated } \\
\text { size }\end{array}$ & $\begin{array}{c}\text { Date of } \\
\text { visit }\end{array}$ & Condition & $\begin{array}{c}\text { Water-quality } \\
\text { sample }\end{array}$ & Comments \\
\hline \multirow[t]{8}{*}{$107-75$} & $34 \mathrm{CDAC}$ & a & $\mathrm{N}$ & -- & -- & -- \\
\hline & 35BBAC & b & $\mathrm{N}$ & -- & -- & -- \\
\hline & 35CABA & $\mathbf{a}$ & $\mathbf{N}$ & - & -- & Dugout. \\
\hline & $35 \mathrm{CBCB}$ & a & $\mathbf{N}$ & -- & -- & -- \\
\hline & 35DDA/B & b & $\mathbf{N}$ & -- & -- & -- \\
\hline & 36ADDD & b & $\mathbf{N}$ & -- & -- & -- \\
\hline & 36BABA & b & $\mathbf{N}$ & - & -. & -. \\
\hline & $\begin{array}{c}\text { 36CDBA \& } \\
\text { CDAB }\end{array}$ & b & $\mathbf{N}$ & -- & -- & -- \\
\hline \multirow[t]{31}{*}{ 107-76- } & IAAAD & b & $\mathbf{N}$ & -- & -- & -- \\
\hline & $1 \mathrm{AACB} / \mathrm{C} / \mathrm{D}$ & c & $\mathbf{N}$ & -- & -- & Dam breached. \\
\hline & $1 \mathrm{ABBC}$ & b & $2-9.93$ & Poor & Yes & -- \\
\hline & 1CCAD/DA & $\mathbf{a}$ & $\mathbf{N}$ & -- & -. & -- \\
\hline & 1DABD & $\mathrm{b}$ & $\mathbf{N}$ & -- & -- &.- \\
\hline & 2DADA & $\mathbf{a}$ & $\mathrm{N}$ & -- & -- & -- \\
\hline & 3ADAC/D & $\mathrm{b}$ & $\mathrm{N}$ & -- & -- & -- \\
\hline & $3 \mathrm{CABA}$ & $\mathrm{b}$ & $\mathrm{N}$ & -- & -- & -- \\
\hline & 3DDDDA/B & $\mathrm{b}$ & $\mathbf{N}$ & -- & -- & -- \\
\hline & 3DDDB & $\mathrm{b}$ & $\mathrm{N}$ & -. & -- & -- \\
\hline & 5ACBD & b & NA & -- & -- & -- \\
\hline & 5BCBA/B & $\mathrm{b}$ & $\mathrm{N}$ & -- & -- & -- \\
\hline & 6CCAA & a & $\mathbf{N}$ & -- & -- & -- \\
\hline & 6CDBB & $\mathrm{b}$ & $\mathbf{N}$ & -- & -- & -. \\
\hline & 7ABAD & $\mathrm{b}$ & $\mathbf{N}$ & - & -- & -- \\
\hline & 8ACAA & $\mathrm{b}$ & $\mathrm{N}$ & - & - & -- \\
\hline & $9 \mathrm{CDBC}$ & a & $\mathrm{N}$ & -- & - & -- \\
\hline & 10ADAA/B & b & $\mathbf{N}$ & -- & -- & -- \\
\hline & 10CCBD & a & $\mathbf{N}$ & -- & -- & -- \\
\hline & 11AADD & a & $2-12-93$ & Below average & No & Dry; overgrown with trees. \\
\hline & $\begin{array}{l}\text { 11DBAA, } \\
\text { ADCC, \& } \\
\text { ACDD }\end{array}$ & c & $\mathbf{N}$ & -- & -. & Dry. \\
\hline & 12BADB & $\mathbf{a}$ & $\mathbf{N}$ & -- & -- & Dugout. \\
\hline & 13CAAC & b & $\mathbf{N}$ & -- & -- & -- \\
\hline & 13DCDC/D & $\mathrm{b}$ & $\mathbf{N}$ & -- & -- & -- \\
\hline & $14 \mathrm{AACC}$ & $\mathrm{b}$ & $2-12-93$ & Poor & No & Dry. \\
\hline & $\begin{array}{c}\text { 14BABB \& } \\
\text { BBAA }\end{array}$ & $\mathrm{b}$ & $\mathbf{N}$ & $\cdots$ & -- & -- \\
\hline & 14BACD & $\mathrm{b}$ & $\mathrm{N}$ & -- & -- & Dam breached. \\
\hline & 14CCBD & $\mathrm{b}$ & $\mathrm{N}$ & -- & -- & -- \\
\hline & 15AAA & $\mathbf{a}$ & $2-11-93$ & Poor & No & Dry; dam breached. \\
\hline & 15BBAB & b & $\mathbf{N}$ & -- & -- & -- \\
\hline & $15 \mathrm{CCCB}$ & $\mathbf{a}$ & $\mathbf{N}$ & -- & -- & - \\
\hline
\end{tabular}


Table 17. Ponds, stock ponds, and dugouts on the Lower Brule Indian Reservation-Continued

\begin{tabular}{|c|c|c|c|c|c|}
\hline Location & $\begin{array}{c}\text { Estimated } \\
\text { size }\end{array}$ & $\begin{array}{l}\text { Date of } \\
\text { visit }\end{array}$ & Condition & $\begin{array}{l}\text { Water-quality } \\
\text { sample }\end{array}$ & Comments \\
\hline 107-76- 15DAC & a & $\mathbf{N}$ & -- & -- & -- \\
\hline 15DDDA & $\mathbf{a}$ & $\mathrm{N}$ & -. & -- & -. \\
\hline $\begin{array}{c}\text { 16BCAB \& } \\
\text { BBDC }\end{array}$ & b & $\mathrm{N}$ & -- & -- & -- \\
\hline 16CACC/D & a & $\mathrm{N}$ & -- & -- & -- \\
\hline 16DABD & $\mathbf{a}$ & $\mathbf{N}$ & -- & -- & Dam possibly breached. \\
\hline $17 \mathrm{ABBB}$ & b & $\mathrm{N}$ & -- & -- & -- \\
\hline $\begin{array}{c}\text { 17CABC \& } \\
\text { CBAD }\end{array}$ & b & NA & -- & -- & -- \\
\hline $19 \mathrm{ABDC}$ & a & $\mathbf{N}$ &.- & -- & -- \\
\hline $\begin{array}{l}\text { 19BDCD \& } \\
\text { BDDC }\end{array}$ & b & $2-11-93$ & Poor & No & Dry. \\
\hline $20 \mathrm{BCCC}$ & b & $\mathbf{N}$ & -- & -- & -- \\
\hline $\begin{array}{c}\text { 20CBAC \& } \\
\text { CBAB }\end{array}$ & b & $\mathrm{N}$ & -- & -- & -- \\
\hline 20DADB & b & $\mathrm{N}$ & -- & -- & - \\
\hline $21 \mathrm{ABDD}$ & a & $\mathbf{N}$ & -- & -- & -- \\
\hline $\begin{array}{r}21 \mathrm{BCAA} \& \\
\mathrm{BDBB} / \mathrm{C}\end{array}$ & b & $\mathrm{N}$ & - & -- & -- \\
\hline $\begin{array}{l}21 \mathrm{CBD}, \\
\mathrm{CCBA}, \& \\
\mathrm{CDBB} / \mathrm{C}\end{array}$ & c & $\mathrm{N}$ & -- & -- & -- \\
\hline 21DCCB & $\mathbf{a}$ & $\mathbf{N}$ & -- & - & -- \\
\hline 22ACBA & b & $\mathrm{N}$ & - & -- & - \\
\hline $\begin{array}{l}\text { 23BBAD, } \\
\text { BABC, \& } \\
\text { BACA/B }\end{array}$ & b & $\mathrm{N}$ & -. & -- & -- \\
\hline $23 \mathrm{CABC}$ & a & $\mathrm{N}$ & -- & -- & Dam partly breached. \\
\hline 23DABB & $\mathbf{a}$ & $2-12-93$ & Above average & No & Frozen to bottom. \\
\hline 23DDDC/D & b & $\mathrm{N}$ & -- & -- & -- \\
\hline $24 \mathrm{CDDB}$ & b & $\mathbf{N}$ & -- & - & -- \\
\hline 24DAAA/D & a & $\mathbf{N}$ & -- & -- & Possibly a dugout. \\
\hline 25BCAD & a & $\mathrm{N}$ &.- & -- & -- \\
\hline $25 \mathrm{CADC}$ & a & $\mathbf{N}$ & -- & -- & Possibly a dugout. \\
\hline 25DBDA & b & $\mathbf{N}$ & -- & -- & Dam possibly breached. \\
\hline $\begin{array}{c}\text { 26ABCD \& } \\
\text { ACBA }\end{array}$ & b & $\mathbf{N}$ & -- & -- & -- \\
\hline $26 \mathrm{CCBB}$ & $\mathbf{a}$ & $\mathrm{N}$ & -- & -- & -- \\
\hline $26 \mathrm{CCC}$ & $\mathbf{a}$ & $\mathbf{N}$ & -- & -- & -- \\
\hline $\begin{array}{r}27 \mathrm{ABB} \& \\
\text { ABDB }\end{array}$ & b & $\mathbf{N}$ & -- & -- & Dam possibly breached. \\
\hline $\begin{array}{c}\text { 27ACA/D \& } \\
\text { ADCB }\end{array}$ & c & $\mathbf{N}$ & -- & -- & -- \\
\hline $27 \mathrm{CABA} / \mathrm{B}$ & b & $\mathbf{N}$ & -- & -- & -- \\
\hline 27DDDC & $\mathbf{a}$ & $\mathbf{N}$ & -- & -- & Possibly a slough. \\
\hline
\end{tabular}


Table 17. Ponds, stock ponds, and dugouts on the Lower Brule Indian Reservation-Continued

\begin{tabular}{|c|c|c|c|c|c|c|}
\hline & Location & $\begin{array}{l}\text { Estimated } \\
\text { size }\end{array}$ & $\begin{array}{l}\text { Date of } \\
\text { visit }\end{array}$ & Condition & $\begin{array}{l}\text { Water-quality } \\
\text { sample }\end{array}$ & Comments \\
\hline \multirow[t]{23}{*}{ 107-76- } & $\begin{array}{c}28 \mathrm{ACCC} / \mathrm{D} \& \\
\mathrm{DBBA}\end{array}$ & $\mathrm{b}$ & $\mathrm{N}$ & -- & -- & - \\
\hline & 28BCAA/D & b & $\mathbf{N}$ & - & -- & -- \\
\hline & 28BDB & $\mathrm{b}$ & $\mathrm{N}$ & -- & -. & Dam breached. \\
\hline & $28 \mathrm{DCDA} / \mathrm{B}$ & a & $\mathrm{N}$ & -- & -- & -- \\
\hline & 29AACB & a & $\mathrm{N}$ & -- & -- & -- \\
\hline & $29 \mathrm{CCBD}$ & a & $\mathrm{N}$ & - & -- & -- \\
\hline & $31 \mathrm{ABAA} / \mathrm{B}$ & b & $2-11-93$ & Poor & No & Dry. \\
\hline & $31 \mathrm{CCCC}$ & a & $\mathrm{N}$ & -- & -- & Dugout. \\
\hline & $31 \mathrm{CCCC} / \mathrm{D}$ & c & $\mathrm{N}$ & - & -- & -- \\
\hline & 31DAAD & a & $2-11-93$ & Poor & No & Dry; overgrown with trees. \\
\hline & 31DDDC & a & $\mathbf{N}$ & - & -- & -- \\
\hline & $32 \mathrm{ABAD}$ & b & $\mathrm{N}$ & -- & -- & -- \\
\hline & $33 \mathrm{ACDD}$ & a & $\mathrm{N}$ & - & -. & -- \\
\hline & 33BBCA & $\mathrm{b}$ & $\mathbf{N}$ & -- & -- & -- \\
\hline & 34ADCA/B & b & $\mathbf{N}$ & -- & -- & -- \\
\hline & 34BBA/D & $\mathrm{b}$ & $\mathrm{N}$ & - & -- & -- \\
\hline & $34 \mathrm{CBDA}$ & a & $\mathrm{N}$ & -- & -- & -- \\
\hline & 35CAA/D & $\mathrm{b}$ & $\mathrm{N}$ & -- & -- & - \\
\hline & $35 \mathrm{CCA}$ & b & $\mathrm{N}$ & -- & -- & -- \\
\hline & 36AACC & a & $\mathrm{N}$ & - & -. & Partly breached. \\
\hline & 36BBCA/D & a & $\mathrm{N}$ & -- & -- & -- \\
\hline & 36BBCC & b & $\mathrm{N}$ & -- & -- & -- \\
\hline & 36DABD & a & $\mathrm{N}$ & -. & -- & Dam possibly breached. \\
\hline \multirow[t]{15}{*}{$107-77-$} & 1BBDD & b & $\mathrm{N}$ & - & -. & -- \\
\hline & $\begin{array}{r}2 \mathrm{CBAC} \& \\
\mathrm{CBDB} / \mathrm{A}\end{array}$ & a & $\mathbf{N}$ & -- & -- & -. \\
\hline & 2DACC/D & b & $\mathrm{N}$ & - & -- & $-\cdot$ \\
\hline & $\begin{array}{l}\text { 3AADC/CD \& } \\
\text { ADBA }\end{array}$ & a & $\mathrm{N}$ & -- & -- & Dam possibly breached. \\
\hline & $11 \mathrm{AADC} / \mathrm{D}$ & b & $\mathrm{N}$ & - & -- & -- \\
\hline & $\begin{array}{c}\text { 11BACC \& } \\
\text { BBDD }\end{array}$ & d & $\mathrm{N}$ & -- & -- & -. \\
\hline & $\begin{array}{l}\text { 11BCCC/D \& } \\
\text { 10ADAD }\end{array}$ & d & $\mathrm{N}$ & - & -- & - \\
\hline & 11DCCA/D & b & $\mathrm{N}$ &.- & -- & -- \\
\hline & 12DCAB/C & a & $\mathrm{N}$ & - & -- & -- \\
\hline & 12DCBA & a & $\mathrm{N}$ & -- & -- & -- \\
\hline & $\begin{array}{c}\text { 13DAAB \& } \\
\text { DABA }\end{array}$ & a & $2-11-93$ & Poor & No & Dry. \\
\hline & 13DABB & a & $\mathrm{N}$ & -- & -- & - \\
\hline & 13DBAB/BA & c & N & -- & -- & -- \\
\hline & 13DDCC & $\mathrm{b}$ & $2-11-93$ & Poor & No & Dam breached. \\
\hline & 14BABB/C & $\mathrm{b}$ & $\mathrm{N}$ & -- & -- & -- \\
\hline
\end{tabular}


Table 17. Ponds, stock ponds, and dugouts on the Lower Brule Indian Reservation-Continued

\begin{tabular}{|c|c|c|c|c|c|c|}
\hline & Location & $\begin{array}{c}\text { Estimated } \\
\text { size }\end{array}$ & $\begin{array}{c}\text { Date of } \\
\text { visit }\end{array}$ & Condition & $\begin{array}{l}\text { Water-quality } \\
\text { sample }\end{array}$ & Comments \\
\hline \multirow[t]{23}{*}{$107-77-$} & $14 \mathrm{CCBA} / \mathrm{D}$ & b & $\mathrm{N}$ & -- & -- & -- \\
\hline & 14DCBA & a & $2-23-93$ & Above average & Yes & - \\
\hline & $\begin{array}{c}15 \mathrm{ABCD} \& \\
\text { ABDC }\end{array}$ & b & $\mathrm{N}$ & -- & -- & -- \\
\hline & 22BAAB & b & $\mathrm{N}$ & -- & -- & Dam breached. \\
\hline & 23AD center & a & $\mathrm{N}$ & -- & - & $-\cdot$ \\
\hline & 23DAAC & a & $\mathrm{N}$ & -- & -- & -- \\
\hline & 23DBBA/B & $\mathrm{b}$ & $2-11-93$ & Above average & Yes & -- \\
\hline & 24CADC & $\mathrm{b}$ & $\mathrm{N}$ & -- & -- & -- \\
\hline & $25 \mathrm{BCDB}$ & $\mathrm{b}$ & $\mathrm{N}$ & -- & -- & -- \\
\hline & 25DBDC & b & $\mathrm{N}$ & -- & -- & -- \\
\hline & $\begin{array}{c}\text { 26ADBC \& } \\
\text { ADCB }\end{array}$ & a & $\mathrm{N}$ & - & -- & -- \\
\hline & 26BDDC & a & $\mathbf{N}$ & -. & -- & Dam breached. \\
\hline & 26CBDC & $\mathrm{b}$ & $\mathbf{N}$ & -- & -- & -- \\
\hline & 26DACD & a & $\mathrm{N}$ & -- & -- & -- \\
\hline & 26DBB & a & $\mathbf{N}$ & -- & -- & Dam breached. \\
\hline & 26DDAD & a & $\mathbf{N}$ & -- & -- & -- \\
\hline & 34AADC & b & $\mathbf{N}$ & -. & -- & -- \\
\hline & 34BBCA & a & $\mathrm{N}$ & -- & -- & Dam breached. \\
\hline & 35BDDA/D & $\mathrm{b}$ & $\mathrm{N}$ & -- & -- & -- \\
\hline & 35DADA/D & a & $\mathbf{N}$ & -- & -- & -- \\
\hline & $35 \mathrm{DBDC}$ & $\mathrm{b}$ & $\mathrm{N}$ & -- & -- & -- \\
\hline & 36AACC/D & a & $\mathbf{N}$ & -- & -- & -- \\
\hline & 36BDDA/B & $\mathrm{b}$ & $\mathbf{N}$ & -- & -- & -- \\
\hline \multirow[t]{18}{*}{ 108-73- } & $6 \mathrm{ABAB}$ & a & $\mathbf{N}$ & -- & -- & -- \\
\hline & 6ABDA & a & $\mathbf{N}$ & -- & -- & -- \\
\hline & 8ADA/D & b & $\mathrm{N}$ & - & -- & Irrigation seepage or drainage pond. \\
\hline & 8DAD & b & $\mathrm{N}$ & -- & -- & do. \\
\hline & 8DBDB & a & $\mathbf{N}$ & -- & -- & do. \\
\hline & $9 \mathrm{CCBA}$ & $\mathrm{b}$ & $\mathbf{N}$ & -- & -- & do. \\
\hline & $9 \mathrm{CDBC}$ & $\mathrm{b}$ & $\mathbf{N}$ & -- & -- & do. \\
\hline & 9CDCB & a & $\mathrm{N}$ & - & -- & do. \\
\hline & $16 \mathrm{ACB}$ & b & $\mathbf{N}$ & -- & -- & do. \\
\hline & 16ACDC & a & $\mathrm{N}$ & -- & -- & do. \\
\hline & 16BAAB & c & $\mathbf{N}$ & $\cdots$ & -- & do. \\
\hline & 16BCDA & a & $\mathbf{N}$ & $\cdots$ & -- & do. \\
\hline & 16BDCC & a & $\mathbf{N}$ & -- & -- & do. \\
\hline & $16 \mathrm{CDAD}$ & a & $\mathrm{N}$ & -. & -- & do. \\
\hline & $16 \mathrm{CDBD}$ & b & $\mathbf{N}$ & -- & -- & do. \\
\hline & 16DAAA/D & b & $\mathbf{N}$ & -- & -- & -- \\
\hline & 17ABCD1 & a & $\mathbf{N}$ & -- & -- & Irrigation seepage or drainage pond. \\
\hline & 17ABCD2 & $\mathbf{a}$ & $\mathbf{N}$ & -- & -- & Irrigation seepage or drainage pond. \\
\hline
\end{tabular}


Table 17. Ponds, stock ponds, and dugouts on the Lower Brule Indian Reservation-Continued

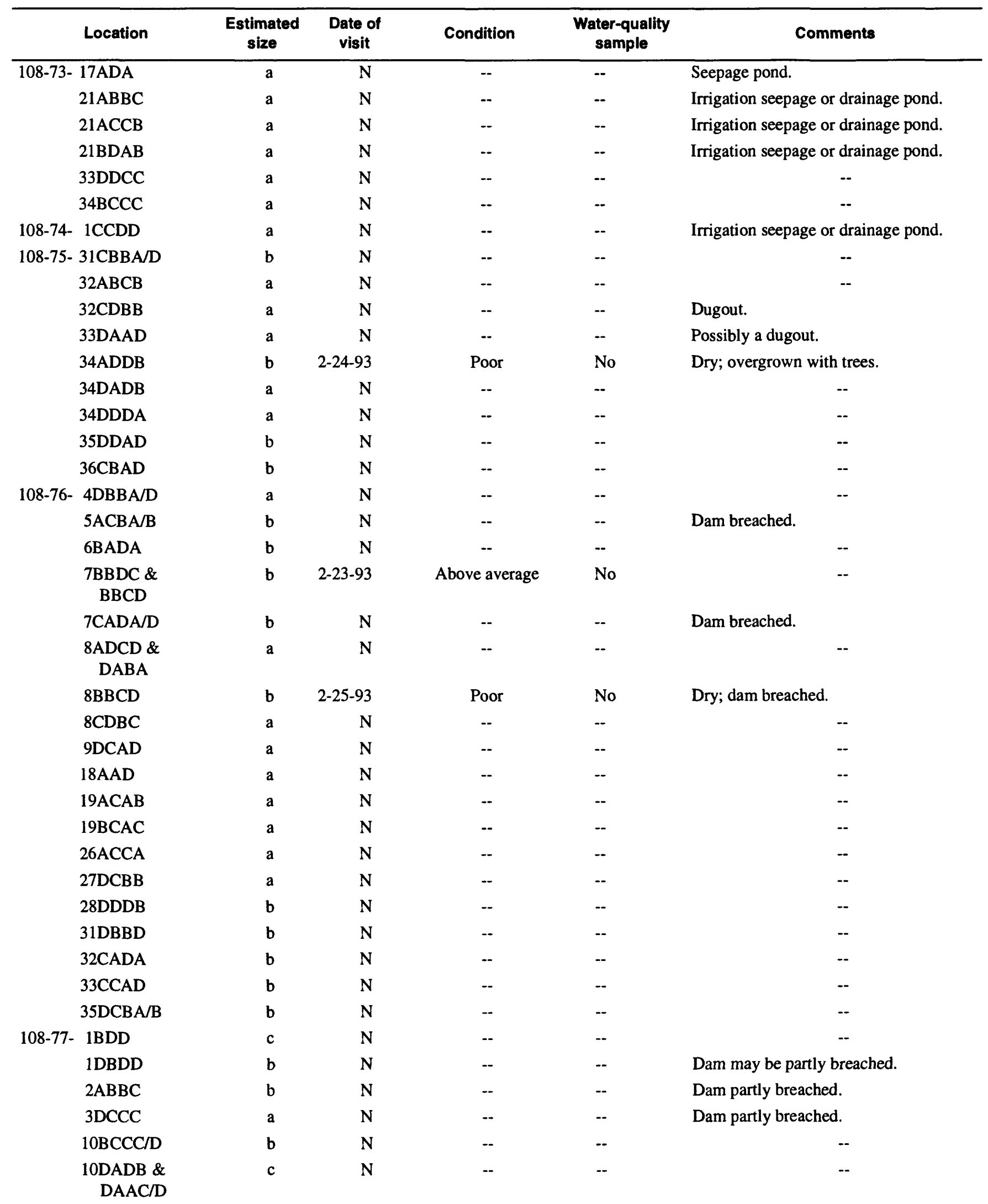


Table 17. Ponds, stock ponds, and dugouts on the Lower Brule Indian Reservation-Continued

\begin{tabular}{|c|c|c|c|c|c|c|}
\hline & Location & $\begin{array}{l}\text { Estimated } \\
\text { size }\end{array}$ & $\begin{array}{l}\text { Date of } \\
\text { visit }\end{array}$ & Condition & $\begin{array}{l}\text { Water-quality } \\
\text { sample }\end{array}$ & Comments \\
\hline 108-77- & 11BBDD & $\bar{a}$ & $\mathbf{N}$ & -- & -- & -- \\
\hline & 11CADC & a & $\mathrm{N}$ & -- & -- & -- \\
\hline & $12 \mathrm{BCCB}$ & b & $\mathbf{N}$ & -- & -- & -- \\
\hline & $12 \mathrm{CCAD}$ & b & $\mathrm{N}$ & -- & -- & -- \\
\hline & $13 \mathrm{ACBC}$ & b & $\mathrm{N}$ & -- & -. & -- \\
\hline & $\begin{array}{l}\text { 13CDCC \& } \\
\text { CDAA }\end{array}$ & $\mathbf{a}$ & $\mathbf{N}$ & -- & -- & -- \\
\hline & 14BDDC & b & $\mathbf{N}$ & -- & -- & -- \\
\hline & 15ABDD & a & $\mathrm{N}$ & -- & -- & -- \\
\hline & 23BADD & a & $N$ & -- & -- & -- \\
\hline & $23 \mathrm{CABC}$ & b & $\mathrm{N}$ & -- & -- & -- \\
\hline & $\begin{array}{l}\text { 23DDAB \& } \\
\text { DADC }\end{array}$ & b & $\mathbf{N}$ & -- & - & -- \\
\hline & 24AAA center & a & $\mathrm{N}$ & -- & -- & -- \\
\hline & 24DCCA/B & a & $\mathrm{N}$ & -- & -- & -- \\
\hline & 27BCAB/C & a & N & -- & -- & -- \\
\hline & $27 \mathrm{CDCD}$ & $\mathrm{b}$ & $\mathbf{N}$ & -- & -- & -- \\
\hline & $\begin{array}{l}\text { 27DCAD \& } \\
\text { DCDA }\end{array}$ & a & $\mathbf{N}$ & -- & -- & -- \\
\hline & 34AABA & a & $\mathbf{N}$ & -- & -- & -- \\
\hline & $34 \mathrm{CDCA}$ & b & $\mathrm{N}$ & -- & -- & -- \\
\hline & 34DAAC & a & $\mathbf{N}$ & -- & -- & -- \\
\hline & 34DADA/B & $\mathbf{a}$ & $\mathrm{N}$ & -. & -- & -- \\
\hline & 34DDAD/C & a & $\mathbf{N}$ & -- & -- & -- \\
\hline & 35DBBA & b & $\mathbf{N}$ & -- & -- & -- \\
\hline & $36 \mathrm{ABAB}$ & a & $\mathbf{N}$ & -- & -- & -- \\
\hline \multirow[t]{6}{*}{ 109-76- } & 6BCAB/C & b & $2-25-93$ & Poor & No & $\begin{array}{l}\text { Dry, overgrown with vegetation; dam } \\
\text { breached. }\end{array}$ \\
\hline & 6CCDA & a & $\mathbf{N}$ & -- & -- & Dry. \\
\hline & $6 \mathrm{CDBC} / \mathrm{CB}$ & a & $\mathbf{N}$ & -- & - & Dam breached. \\
\hline & 7AACA/B & a & $2-25-93$ & Poor & No & Dugout; dry, overgrown with vegetation. \\
\hline & 7ABAD & a & $2-25-93$ & Poor & No & Dugout; dry, overgrown with vegetation. \\
\hline & $\begin{array}{l}7 \mathrm{CCBC} \& \\
12 \mathrm{DDAD}\end{array}$ & a & $\mathbf{N}$ & -- & -- & -- \\
\hline \multirow[t]{9}{*}{ 109-76- } & 8BCBD & a & $2-25-93$ & Poor & No & Dry, overgrown with vegetation. \\
\hline & 9DDDC & $\mathrm{b}$ & $2-25-93$ & Poor & No & Dry, overgrown with vegetation. \\
\hline & 10DCC/D & a & $\mathbf{N}$ & -- & -- & Dam breached. \\
\hline & $14 \mathrm{CCDB}$ & $\mathbf{a}$ & $\mathbf{N}$ & -- & -- & Dugout. \\
\hline & 14CDDD & a & N & -- & -- & Dam possibly breached. \\
\hline & $\begin{array}{l}\text { 17CAAC \& } \\
\text { CABD }\end{array}$ & b & $\mathbf{N}$ & -- & -- & -- \\
\hline & 18DADD & b & $\mathbf{N}$ & -- & -- & -- \\
\hline & 19BCAB & $\mathrm{b}$ & $\mathbf{N}$ & -- & -- & -- \\
\hline & 19BCBD & b & $\mathbf{N}$ & -- & -- & - \\
\hline
\end{tabular}


Table 17. Ponds, stock ponds, and dugouts on the Lower Brule Indian Reservation-Continued

\begin{tabular}{|c|c|c|c|c|c|c|}
\hline & Location & $\begin{array}{l}\text { Estimated } \\
\text { size }\end{array}$ & $\begin{array}{l}\text { Date of } \\
\text { visit }\end{array}$ & Condition & $\begin{array}{l}\text { Water-quality } \\
\text { sampie }\end{array}$ & Comments \\
\hline \multirow[t]{11}{*}{ 109-76- } & $20 \mathrm{ACBB}$ & $\mathrm{b}$ & $\mathbf{N}$ & -- & - & -- \\
\hline & $\begin{array}{c}\text { 21DCBD \& } \\
\text { DCCA }\end{array}$ & $\mathrm{b}$ & $\mathbf{N}$ & -- & -- & -- \\
\hline & $\begin{array}{c}\text { 22BBCD/DC \& } \\
\text { BCBA/AB }\end{array}$ & a & $\mathbf{N}$ & -- & -- & -- \\
\hline & 27ACAA & a & $\mathbf{N}$ & -- & -- & -- \\
\hline & 28CDAC/DB & $\mathbf{a}$ & $\mathbf{N}$ & -- & -- & -- \\
\hline & 29BBBB/C & c & $2-24-93$ & -- & Yes & -- \\
\hline & $30 \mathrm{ACDB} / \mathrm{C}$ & $\mathbf{a}$ & N & -- & -- & - \\
\hline & $\begin{array}{c}\text { 30BBCA \& } \\
\text { BBDC }\end{array}$ & $\mathrm{b}$ & $\mathbf{N}$ & -- & -- & -- \\
\hline & $31 \mathrm{CABC}$ & $\mathrm{b}$ & $\mathrm{N}$ & -- & -- & -- \\
\hline & 32BBDC & $\mathrm{b}$ & $\mathbf{N}$ & -- & -- & -- \\
\hline & $\begin{array}{c}\text { 33CBDD \& } \\
\text { CCAA }\end{array}$ & c & $2-24-93$ & Above average & Yes & -- \\
\hline \multirow[t]{25}{*}{ 109-77- } & $1 \mathrm{DD}$ & -- & $2-25-93$ & Poor & No & Dry, overgrown with vegetation. \\
\hline & 2ADCA/B & b & $\mathbf{N}$ & -- & -- & -- \\
\hline & 2DDCC/D & b & $\mathbf{N}$ & -- & -- & -- \\
\hline & 3BDDB/C & b & $\mathbf{N}$ & -- & -- & -- \\
\hline & 3DABC/D & a & $2-25-93$ & Poor & No & Dugout; dry. \\
\hline & 10AAC & b & $\mathbf{N}$ & -- & -- & -- \\
\hline & $12 \mathrm{AABA}$ & a & $\mathrm{N}$ & -- & -- & Possibly a stock pond. \\
\hline & 12BADB & $\mathrm{b}$ & $\mathbf{N}$ & -- & -- & -- \\
\hline & 13ADCA & $\mathrm{b}$ & $\mathbf{N}$ & -- & -- & -- \\
\hline & 13DBAC & b & $\mathbf{N}$ & -- & -- & -- \\
\hline & 14BDDC & b & $\mathbf{N}$ & -- & - & -- \\
\hline & $15 \mathrm{BCAA}$ & $\mathrm{b}$ & $\mathbf{N}$ & -- & -- & -- \\
\hline & $15 \mathrm{CCDA}$ & c & $\mathbf{N}$ & -- & -- & Dam breached. \\
\hline & 15DAAB & b & $\mathbf{N}$ & -- & -- & -- \\
\hline & $22 \mathrm{CDAD}$ & b & $\mathbf{N}$ & -- & -- & Possibly a dugout. \\
\hline & 23DDDC & a & $\mathbf{N}$ & -- & -- & - \\
\hline & 24ADAC & a & $\mathrm{N}$ & -- & -- & -- \\
\hline & 24BCCA/B & a & $\mathrm{N}$ & -- & -- & -- \\
\hline & 24DDCD & b & $\mathbf{N}$ & -- & -- & -- \\
\hline & $25 \mathrm{BBAB} / \mathrm{C}$ & b & $\mathbf{N}$ & -- & -- & Dam breached. \\
\hline & 26ABAD & a & $\mathbf{N}$ & -- & -- & Dam possibly breached. \\
\hline & $27 \mathrm{ABBC}$ & a & $\mathbf{N}$ & -- & -- & Dugout. \\
\hline & $35 \mathrm{CABA}$ & a & $\mathrm{N}$ & -- & -- & -- \\
\hline & 36BCCA & b & $\mathrm{N}$ & -- & -- & -- \\
\hline & $36 \mathrm{BCCC}$ & b & $\mathbf{N}$ & -- & -- & -- \\
\hline
\end{tabular}

